\title{
Vinyl thianthrenium tetrafluoroborate: A practical and versatile vinylating reagent made from ethylene
}

\author{
Fabio Juliá, Jiyao Yan, Fritz Paulus and Tobias Ritter* \\ Max-Planck-Institut für Kohlenforschung \\ Kaiser-Wilhelm Platz 1, 45470 Mülheim an der Ruhr, Germany \\ *E-mail: ritter@mpi-muelheim.mpg.de
}




\section{TABLE OF CONTENTS}

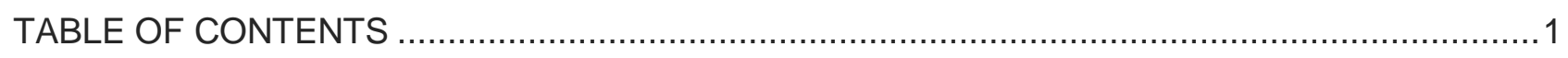

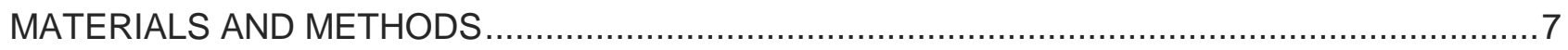

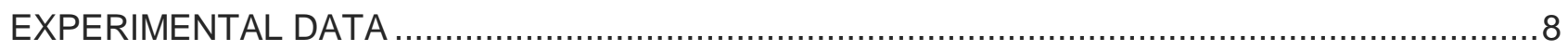

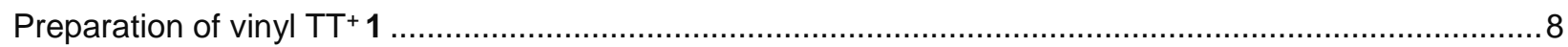

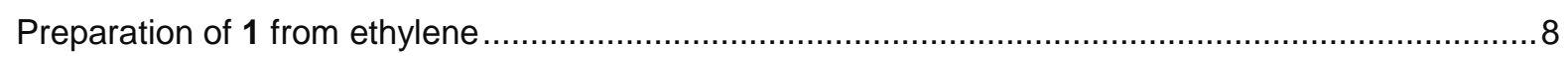

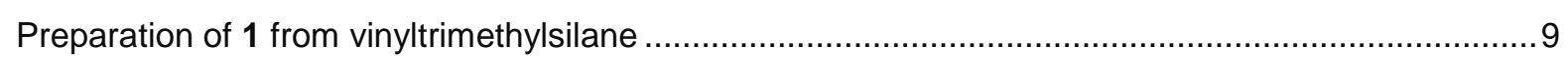

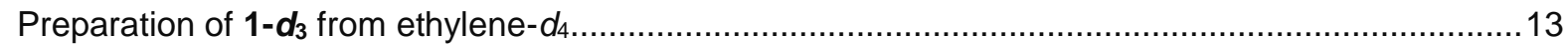

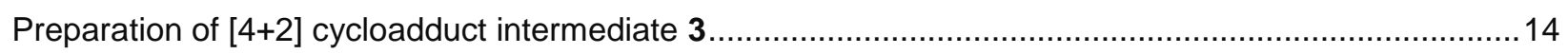

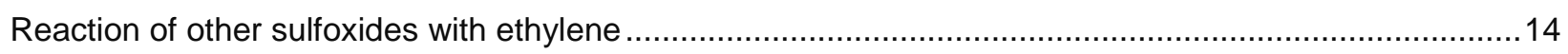

Reaction of diphenylsulfoxide with ethylene ....................................................................... 14

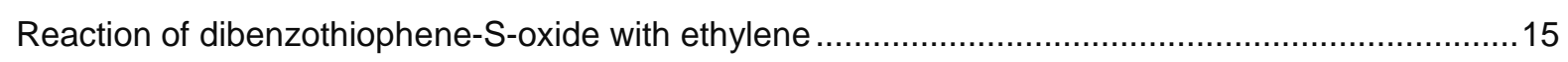

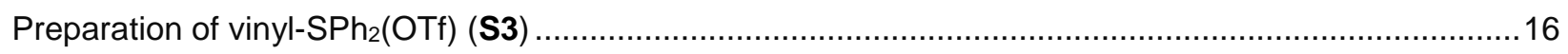

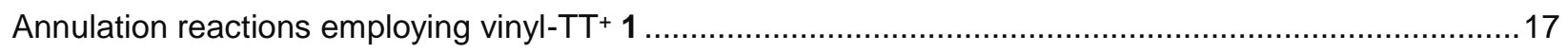

Spiro[cyclopropane-1,3'-indolin]-2'-one (5) …................................................................ 17

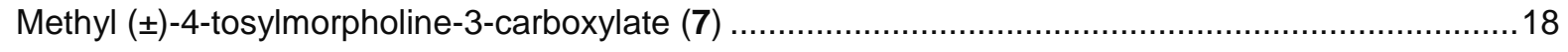

Ethyl ( \pm )-2-phenyl-1-tosylazetidine-2-carboxylate (9) …....................................................... 18

( \pm -3-Tosyl-7-oxa-3-azabicyclo[4.1.0]heptane (11) ............................................................ 19

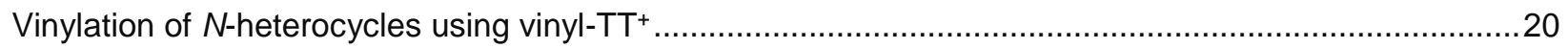

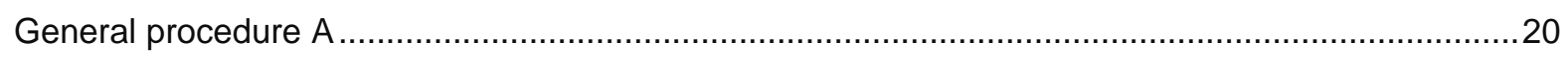

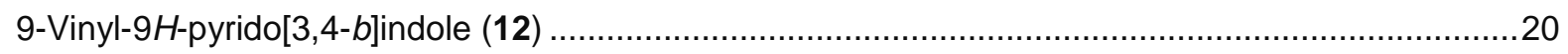

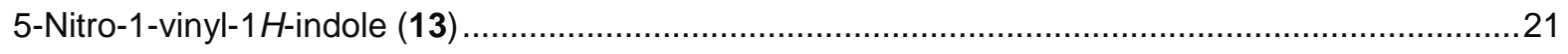

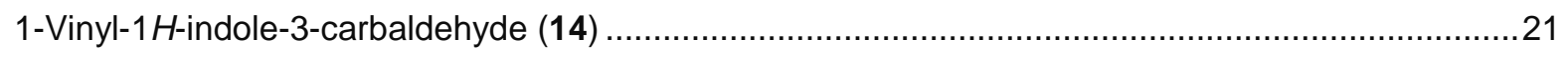

Ethyl 3-amino-1-vinyl-1H-pyrazole-4-carboxylate (15-I) and ethyl 5-amino-1-vinyl-1H-pyrazole-4-

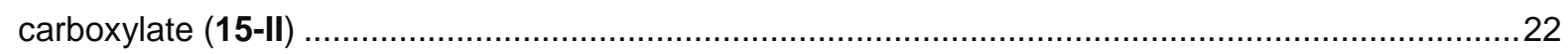

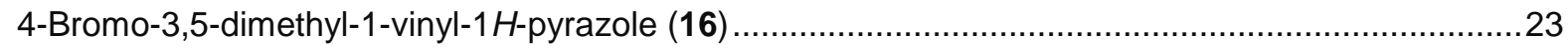

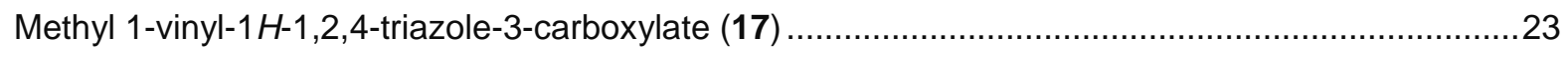

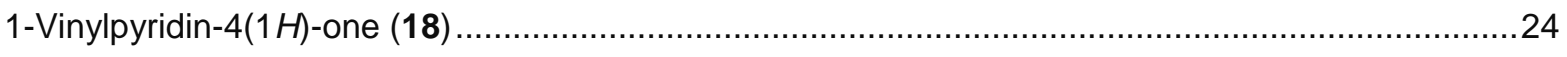

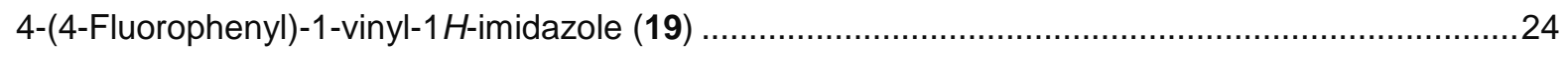

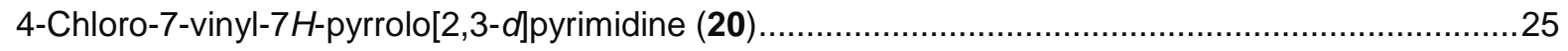

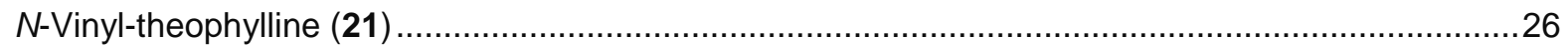

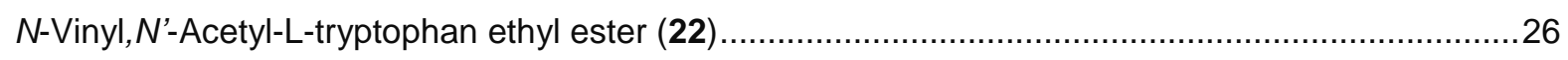

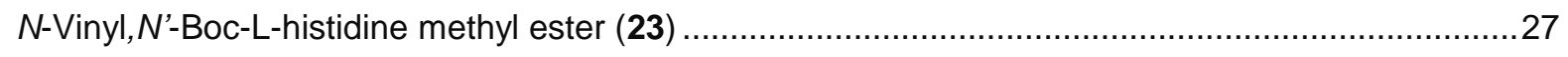

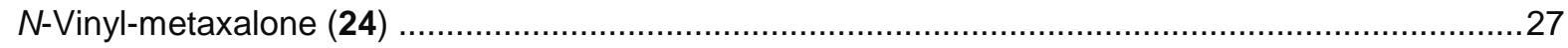




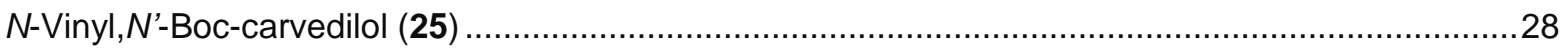

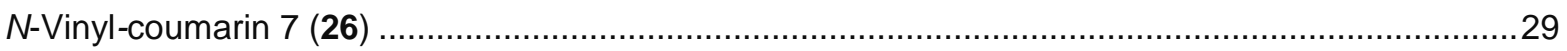

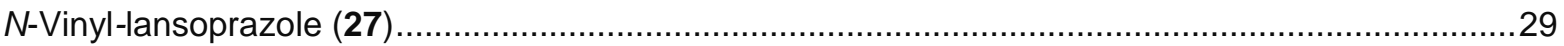

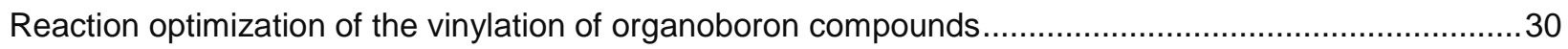

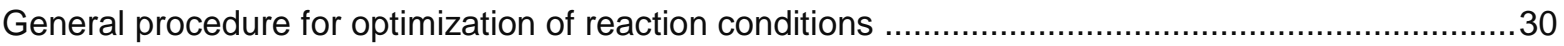

Table S1: Optimization of yield as a function of catalyst............................................................... 30

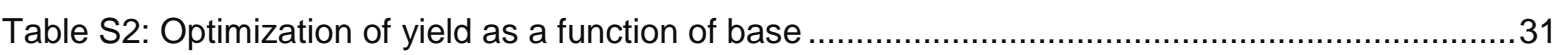

Table S3: Optimization of yield as a function of concentration .......................................................32

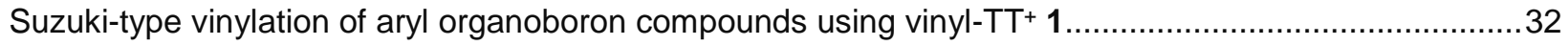

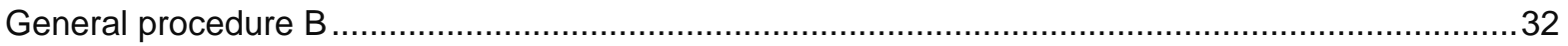

General procedure for the vinylation of organoboron compounds using a Schlenk line ...................33

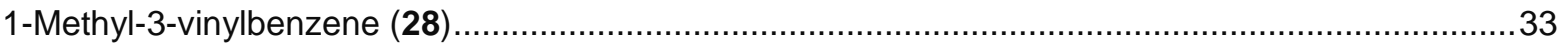

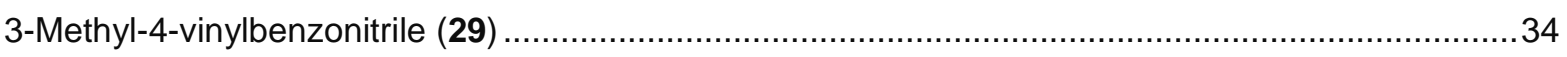

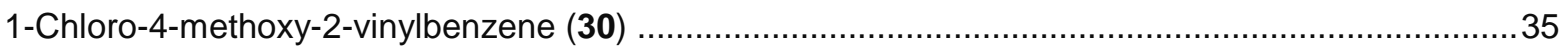

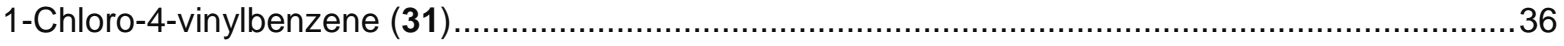

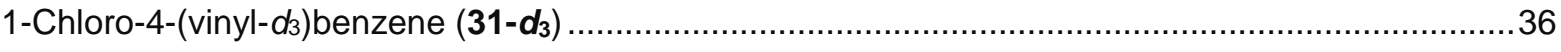

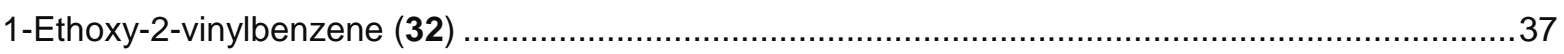

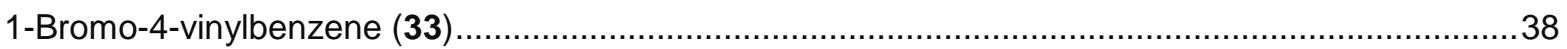

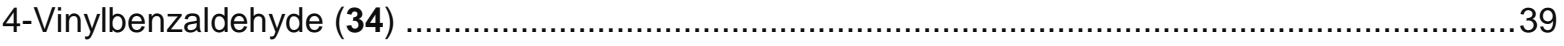

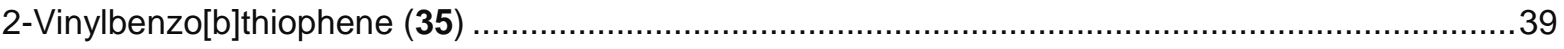

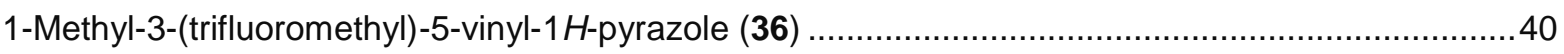

1-Chloro-4-(trifluoromethyl)-2-((2-vinylbenzyl)oxy)benzene (37) .................................................. 41

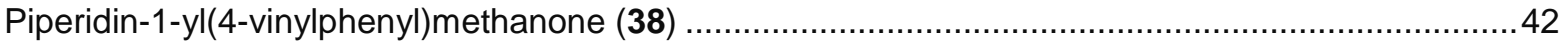

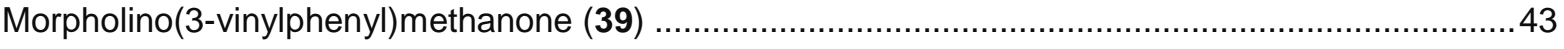

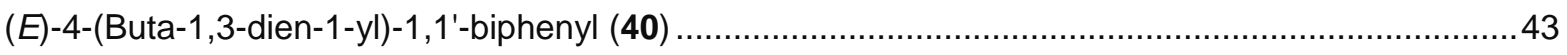

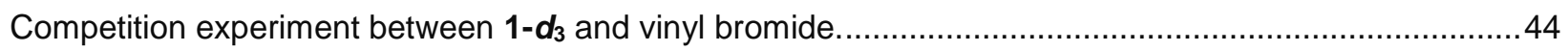

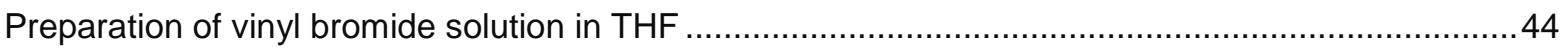

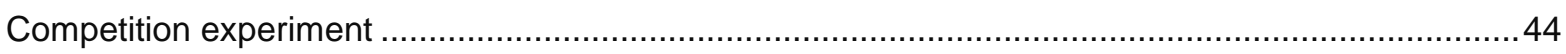

Comparison on the performance of 1 and vinyl-SPh$(\mathrm{OTf})$ in Suzuki-type reactions. ..............................46

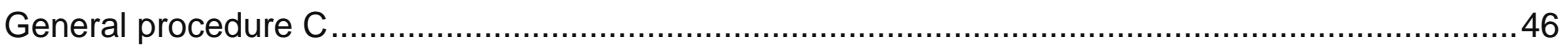

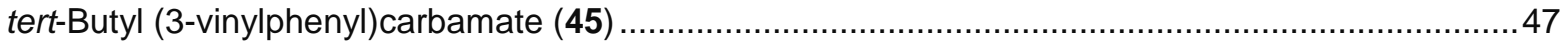

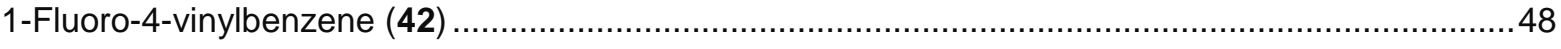

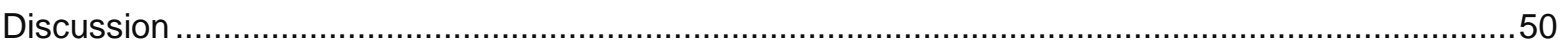

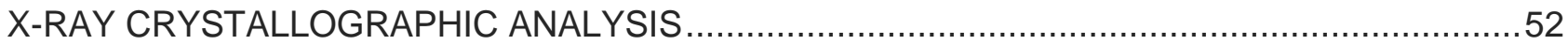

S-vinyl-thianthrenium tetrafluoroborate (vinyl-TT+, 1) (CCDC 2075820) …..........................................52 
Experimental

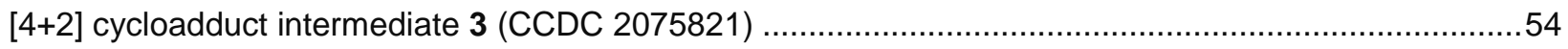

Experimental

SPECTROSCOPIC DATA

${ }^{1} \mathrm{H}$ NMR of vinyl TT+ (1) .57

${ }^{13} \mathrm{C}$ NMR of vinyl TT+ $(\mathbf{1})$ .58

${ }^{19} \mathrm{~F}$ NMR of vinyl TT+ $(\mathbf{1})$ .59

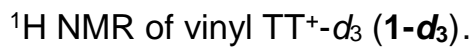
60

${ }^{2} \mathrm{H}$ NMR of vinyl TT+ ${ }^{+} d_{3}\left(\mathbf{1}-\boldsymbol{d}_{3}\right)$ 61

${ }^{13} \mathrm{C}$ NMR of vinyl TT+ ${ }^{+} d_{3}\left(\mathbf{1}-\boldsymbol{d}_{3}\right)$ 61

${ }^{19} \mathrm{~F}$ NMR of vinyl TT+- $d_{3}\left(\mathbf{1}-\boldsymbol{d}_{\mathbf{3}}\right)$ 63

${ }^{1} \mathrm{H}$ NMR of the [4+2] cycloadduct intermediate 3 64

${ }^{13} \mathrm{C}$ NMR of the [4+2] cycloadduct intermediate 3 .65

${ }^{19} \mathrm{~F}$ NMR of the [4+2] cycloadduct intermediate 3 .66

${ }^{1} \mathrm{H}$ NMR of spiro[cyclopropane-1,3'-indolin]-2'-one (5). .67

${ }^{13} \mathrm{C}$ NMR of spiro[cyclopropane-1,3'-indolin]-2'-one (5) 68

${ }^{1} \mathrm{H}$ NMR of methyl ( \pm )-4-tosylmorpholine-3-carboxylate (7) .69

${ }^{13} \mathrm{C}$ NMR of methyl ( \pm )-4-tosylmorpholine-3-carboxylate (7) .70

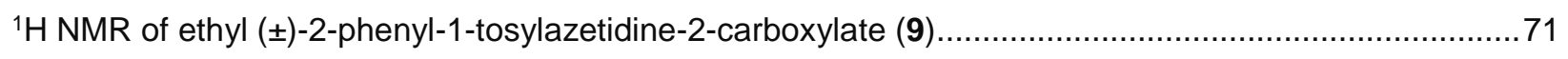

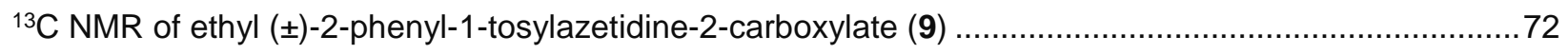

${ }^{1} \mathrm{H}$ NMR of ( \pm -3-tosyl-7-oxa-3-azabicyclo[4.1.0]heptane (11) ....................................................... 73

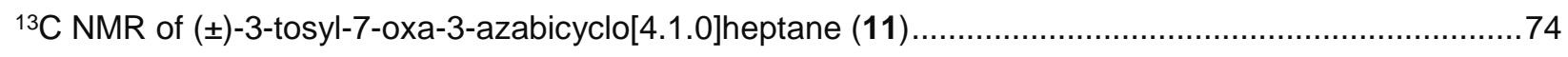

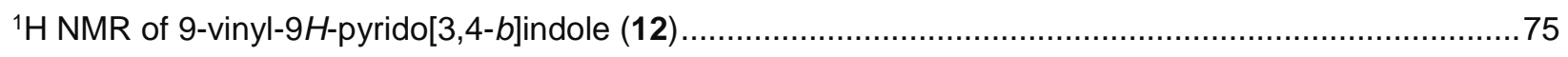

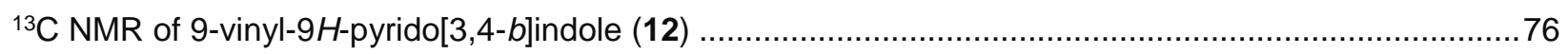

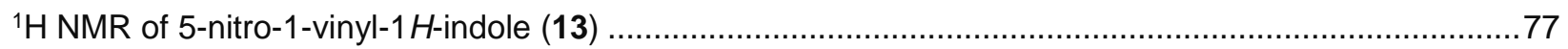

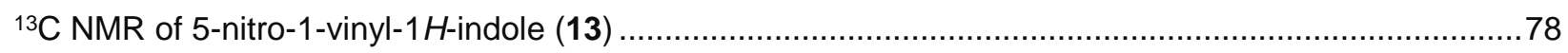

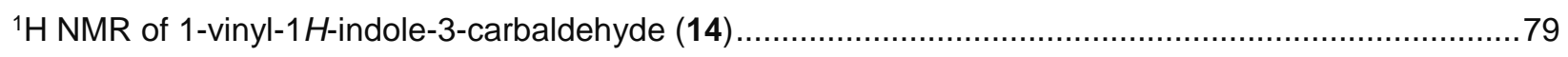

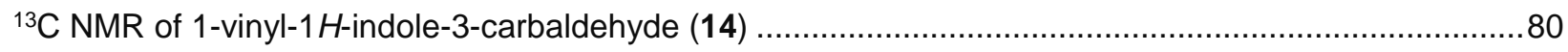

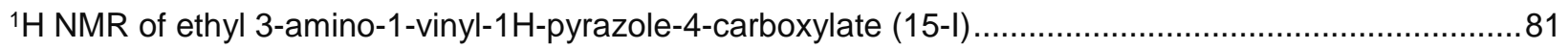


${ }^{13} \mathrm{C}$ NMR of ethyl 3-amino-1-vinyl-1H-pyrazole-4-carboxylate (15-I)

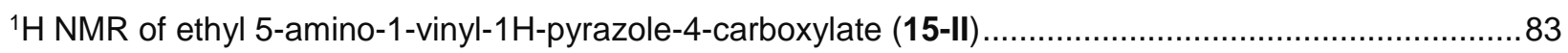

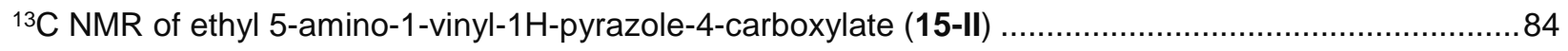

${ }^{1} \mathrm{H}$ NMR of 4-bromo-3,5-dimethyl-1-vinyl-1 $\mathrm{H}$-pyrazole (16) .......................................................... 85

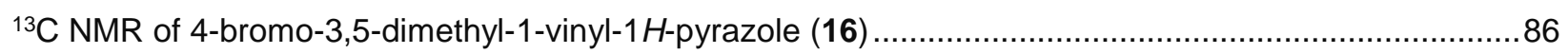

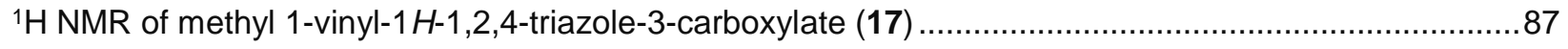

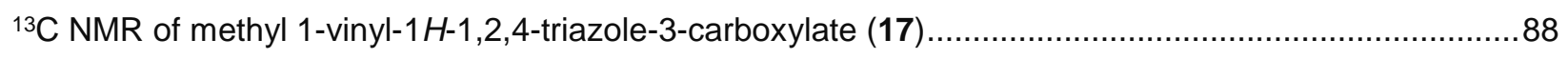

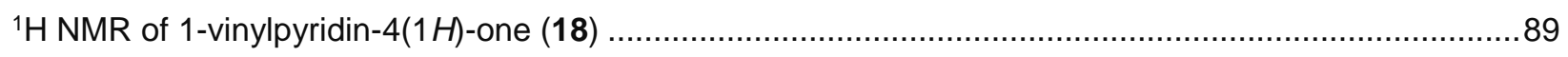

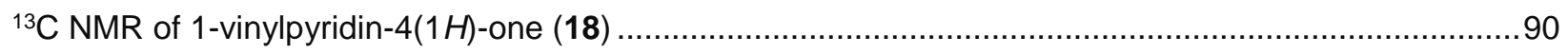

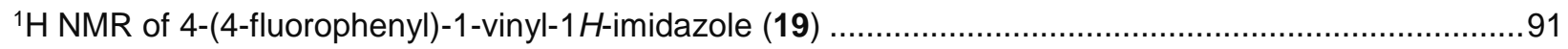

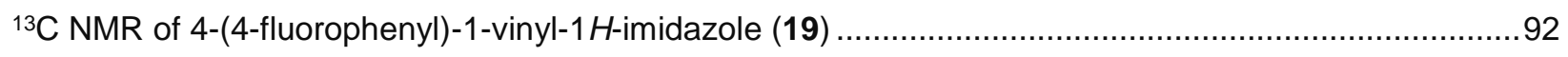

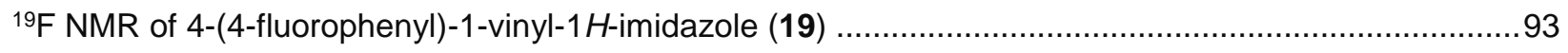

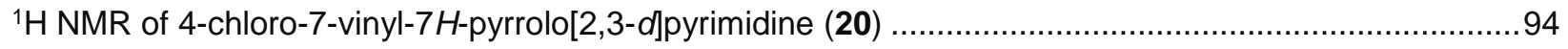

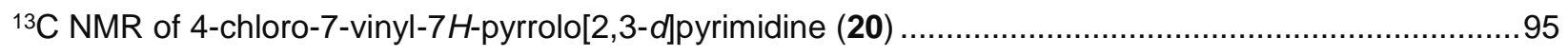

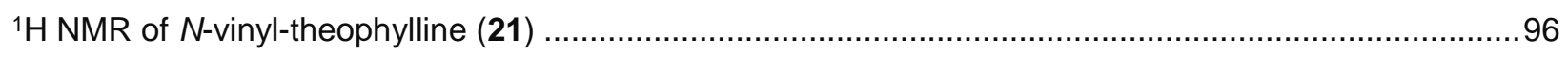

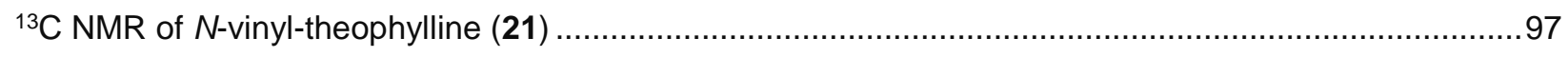

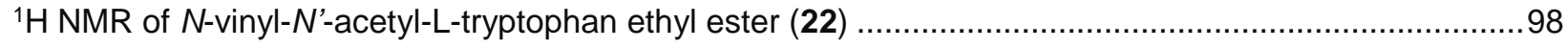

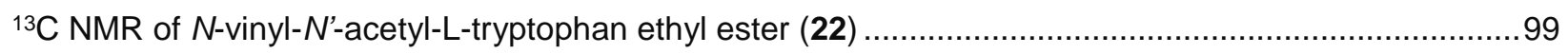

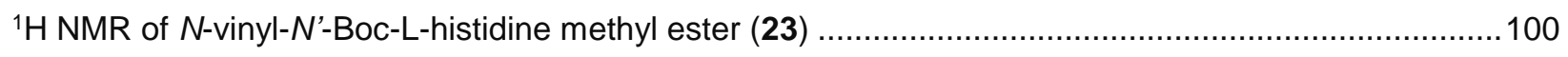

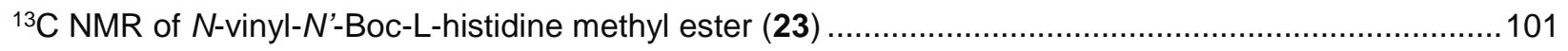

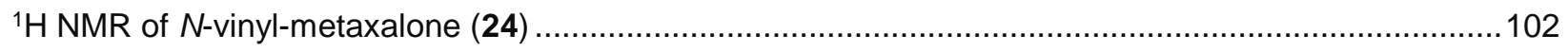

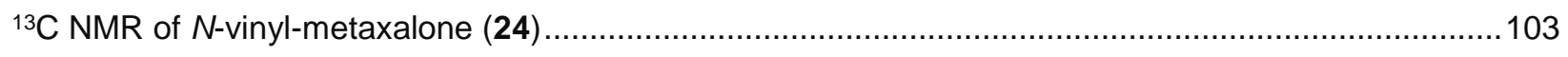

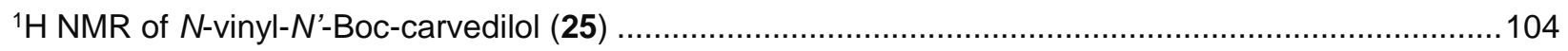

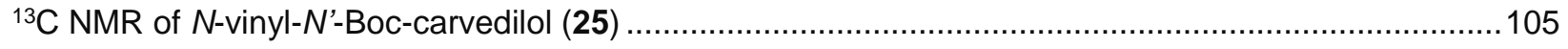

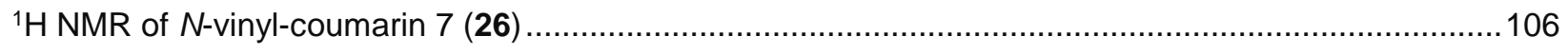

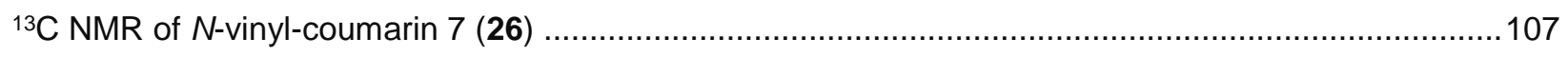

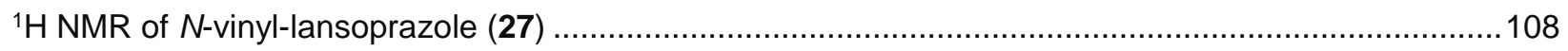

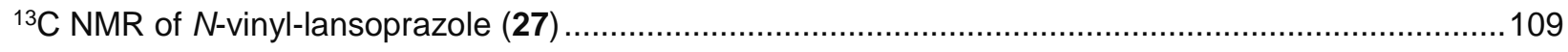

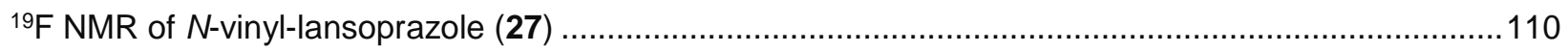


${ }^{1} \mathrm{H}$ NMR of 3-methyl-4-vinylbenzonitrile (29)

${ }^{13} \mathrm{C}$ NMR of 3-methyl-4-vinylbenzonitrile (29)

${ }^{1} \mathrm{H}$ NMR of 1-chloro-4-methoxy-2-vinylbenzene (30)

${ }^{13} \mathrm{C}$ NMR of 1-chloro-4-methoxy-2-vinylbenzene (30)

${ }^{1} \mathrm{H}$ NMR of 1-chloro-4-vinylbenzene (31)

${ }^{13} \mathrm{C}$ NMR of 1-chloro-4-vinylbenzene (31)

${ }^{1} \mathrm{H}$ NMR of 1 -chloro-4-(vinyl- $\left.d_{3}\right)$ benzene $\left(\mathbf{3 1}-\boldsymbol{d}_{3}\right)$.

${ }^{2} \mathrm{H}$ NMR of 1 -chloro-4-(vinyl- $\left.d_{3}\right)$ benzene $\left(\mathbf{3 1}-\boldsymbol{d}_{3}\right)$.

${ }^{13} \mathrm{C}$ NMR of 1 -chloro-4-(vinyl- $\left.d_{3}\right)$ benzene $\left(\mathbf{3 1}-\boldsymbol{d}_{3}\right)$

${ }^{1} \mathrm{H}$ NMR of 1-ethoxy-2-vinylbenzene (32).

${ }^{13} \mathrm{C}$ NMR of 1-ethoxy-2-vinylbenzene (32)

${ }^{1} \mathrm{H}$ NMR of 1-bromo-4-vinylbenzene (33)

${ }^{13} \mathrm{C}$ NMR of 1-bromo-4-vinylbenzene (33).

${ }^{1} \mathrm{H}$ NMR of 4-vinylbenzaldehyde (34)

${ }^{13} \mathrm{C}$ NMR of 4-vinylbenzaldehyde (34)

${ }^{1} \mathrm{H}$ NMR of 2-vinylbenzo[b]thiophene (35) 126

${ }^{13} \mathrm{C}$ NMR of 2-vinylbenzo[b]thiophene (35)

${ }^{1} \mathrm{H}$ NMR of 1-methyl-3-(trifluoromethyl)-5-vinyl-1 H-pyrazole (36) ....................................................128

${ }^{13} \mathrm{C}$ NMR of 1 -methyl-3-(trifluoromethyl)-5-vinyl-1 H-pyrazole (36) ................................................129

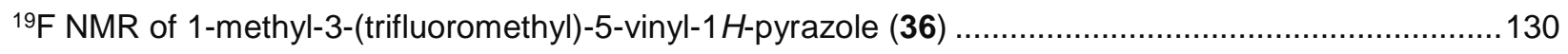

${ }^{1} \mathrm{H}$ NMR of 1-chloro-4-(trifluoromethyl)-2-((2-vinylbenzyl)oxy)benzene (37) .......................................131

${ }^{13} \mathrm{C}$ NMR of 1-chloro-4-(trifluoromethyl)-2-((2-vinylbenzyl)oxy)benzene (37) ..................................... 131

${ }^{19} \mathrm{~F}$ NMR of 1-chloro-4-(trifluoromethyl)-2-((2-vinylbenzyl)oxy)benzene (37) .....................................133

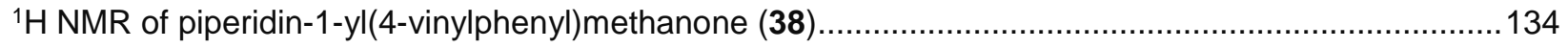

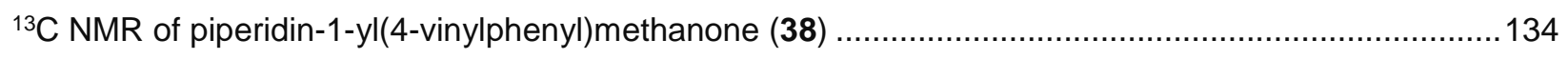

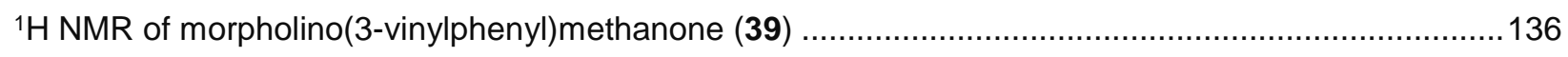

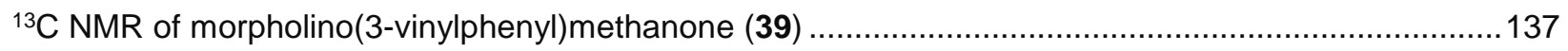

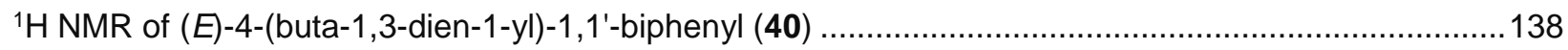

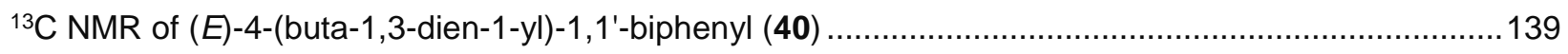




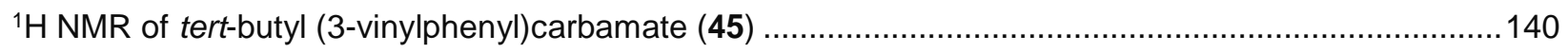

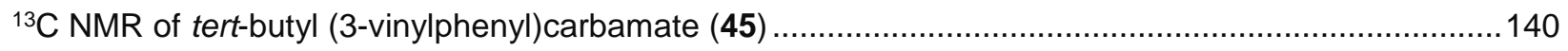

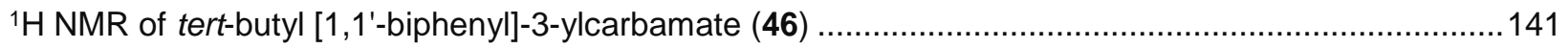

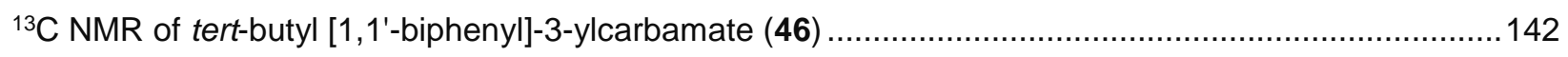

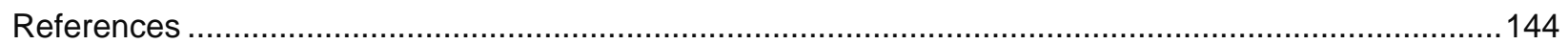




\section{MATERIALS AND METHODS}

All reactions were carried out under an ambient atmosphere unless otherwise stated and monitored by thinlayer chromatography (TLC). Air- and moisture-sensitive manipulations were performed using standard Schlenk- and glove-box techniques under an atmosphere of argon or dinitrogen. High-resolution mass spectra were obtained using $Q$ Exactive Plus from Thermo. Concentration under reduced pressure was performed by rotary evaporation at $25-40{ }^{\circ} \mathrm{C}$ at an appropriate pressure. When it was not removed by means of aqueous workup, DMSO was removed in a Biotage V10 evaporator. Purified compounds were further dried under vacuum $\left(10^{-6}-10^{-3}\right.$ bar). Yields refer to purified and spectroscopically pure compounds, unless otherwise stated.

\section{Solvents}

Dichloromethane, dimethylsulfoxide, acetonitrile and diethyl ether were purchased from Fisher Scientific $\mathrm{GmbH}$ and used as received. Anhydrous solvents were obtained from Phoenix Solvent Drying Systems. All deuterated solvents were purchased from Euriso-Top.

\section{Chromatography}

Thin layer chromatography (TLC) was performed using EMD TLC plates pre-coated with $250 \mu \mathrm{m}$ thickness silica gel $60 \mathrm{~F}_{254}$ plates and visualized by fluorescence quenching under UV light and $\mathrm{KMnO}_{4}$ stain. Flash column chromatography was performed using silica gel (40-63 $\mu \mathrm{m}$ particle size) purchased from Geduran®.

\section{Spectroscopy and Instruments}

NMR spectra were recorded on a Bruker Ascend ${ }^{\mathrm{TM}} 500$ spectrometer operating at $500 \mathrm{MHz}, 471 \mathrm{MHz}$ and $126 \mathrm{MHz}$, for ${ }^{1} \mathrm{H},{ }^{19} \mathrm{~F}$ and ${ }^{13} \mathrm{C}$ acquisitions, respectively; or on a Varian Unity/Inova 600 spectrometer operating at $600 \mathrm{MHz}$ and $151 \mathrm{MHz}$ for ${ }^{1} \mathrm{H}$ and ${ }^{13} \mathrm{C}$ acquisitions, respectively; or on a Bruker Ultrashield ${ }^{\mathrm{TM}} 300$ spectrometer operating at $300 \mathrm{MHz}, 282 \mathrm{MHz}$ and $75 \mathrm{MHz}$ for ${ }^{1} \mathrm{H},{ }^{19} \mathrm{~F}$ and ${ }^{13} \mathrm{C}$ acquisitions, respectively. Chemical shifts are reported in ppm with the solvent residual peak as the internal standard. For ${ }^{1} \mathrm{H}$ NMR: $\mathrm{CDCl}_{3}, \delta$ 7.26; $\mathrm{CD}_{3} \mathrm{CN}, \delta$ 1.96; $\mathrm{CD}_{2} \mathrm{Cl}_{2}, \delta$ 5.32; For ${ }^{13} \mathrm{C} \mathrm{NMR}: \mathrm{CDCl}_{3}, \delta 77.16 ; \mathrm{CD}_{3} \mathrm{CN}, \delta 1.32 ; \mathrm{CD}_{2} \mathrm{Cl}_{2}, \delta$ 53.84. ${ }^{19} \mathrm{~F}$ NMR spectra were referenced using a unified chemical shift scale based on the ${ }^{1} \mathrm{H}$ resonance of tetramethylsilane ( $1 \% \mathrm{v} / \mathrm{v}$ solution in the respective solvent). Data is reported as follows: $s=\operatorname{singlet}, d=$ doublet, $\mathrm{t}=$ triplet, $\mathrm{q}=$ quartet, quin = quintet, sext = sextet, sept = septet, $\mathrm{m}=$ multiplet, $\mathrm{bs}=$ broad singlet; coupling constants in $\mathrm{Hz}$; integration.

\section{Starting materials}

Chemicals were used as received from commercial suppliers, unless otherwise stated. Thianthrene $S-$ oxide, ${ }^{[1]} \mathrm{N}$-tosyl DL-serine methyl ester, ${ }^{[2]} 4$-methyl- $N$-(3-oxopropyl)benzenesulfonamide (tosylation of 3amino-1-propanol,[3] then oxidation $\left.{ }^{[4]}\right), N$-tosyl DL-phenylglycine ethyl ester, ${ }^{[5]}$ dibenzothiophene-S-oxide, ${ }^{[6]}$ and $\mathrm{N}$-Boc-carvedilo[[]] were synthesized according to literature reports. Zinc triflate was dried in a desiccator over $\mathrm{P}_{2} \mathrm{O}_{5}$. 


\section{EXPERIMENTAL DATA}

\section{Preparation of vinyl $\mathrm{TT}^{+}+1$}

Preparation of 1 from ethylene
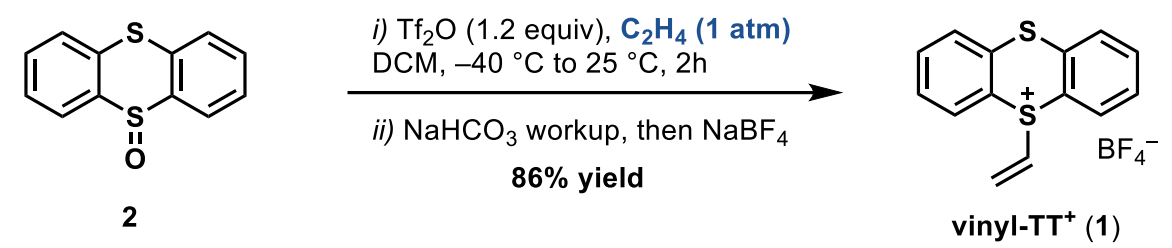

To a round-bottom flask equipped with a stirring bar were added thianthrene-S-oxide 2 (11.7 g, $50.3 \mathrm{mmol}$, 1.00 equiv.) and $400 \mathrm{~mL}$ of $\mathrm{DCM}(\mathrm{c}=0.125 \mathrm{M})$. The flask was capped with a rubber septum and cooled down to $-40^{\circ} \mathrm{C}$. Through the solution was then bubbled ethylene gas for 15 minutes, after which a balloon filled with ethylene was connected to the flask to maintain the ethylene atmosphere throughout the reaction. Triflic anhydride (10.2 mL, $17.0 \mathrm{~g}, 60.4 \mathrm{mmol}, 1.20$ equiv.) was added dropwise to the reaction, and a dark purple suspension was progressively formed [Note: in large scale experiments the amount of precipitate formed can complicate an appropriate stirring. Additional portions of DCM may be added to aid stirring]. After 20 minutes, the cooling bath was removed and the mixture was stirred for 1.5 hour at $25^{\circ} \mathrm{C}$. The ethylene balloon and the rubber septum were removed, and sat. aqueous $\mathrm{NaHCO}_{3}(400 \mathrm{~mL})$ was added carefully. The mixture was vigorously shaken in a separation funnel, phases were separated and the aqueous layer was extracted with DCM $(2 \times 200 \mathrm{~mL})$. All organic phases were combined, partially concentrated $(\sim 300 \mathrm{~mL})$, washed with aqueous solutions of $5 \% \mathrm{NaBF}_{4}(3 \times 100 \mathrm{~mL})$, dried over $\mathrm{MgSO}_{4}$, filtered, and the solvent evaporated under reduced pressure. The crude material was dissolved in $\mathrm{DCM}(60 \mathrm{~mL})$, and $\mathrm{Et}_{2} \mathrm{O}(300 \mathrm{~mL})$ was subsequently added while stirring, causing the precipitation of a solid that was collected by filtration, washed with $\mathrm{Et}_{2} \mathrm{O}$ $(3 \times 20 \mathrm{~mL})$, and dried under vacuum to afford 1 as an off-white solid (14.37 g, $43.52 \mathrm{mmol}, 86 \%)$. [Note: Colored trace impurities coming from old bottles of triflic anhydride might result in 1 as a pale yellow solid (even at $>99 \%$ purity), but have no observable effect on the reactivity reported in this work].

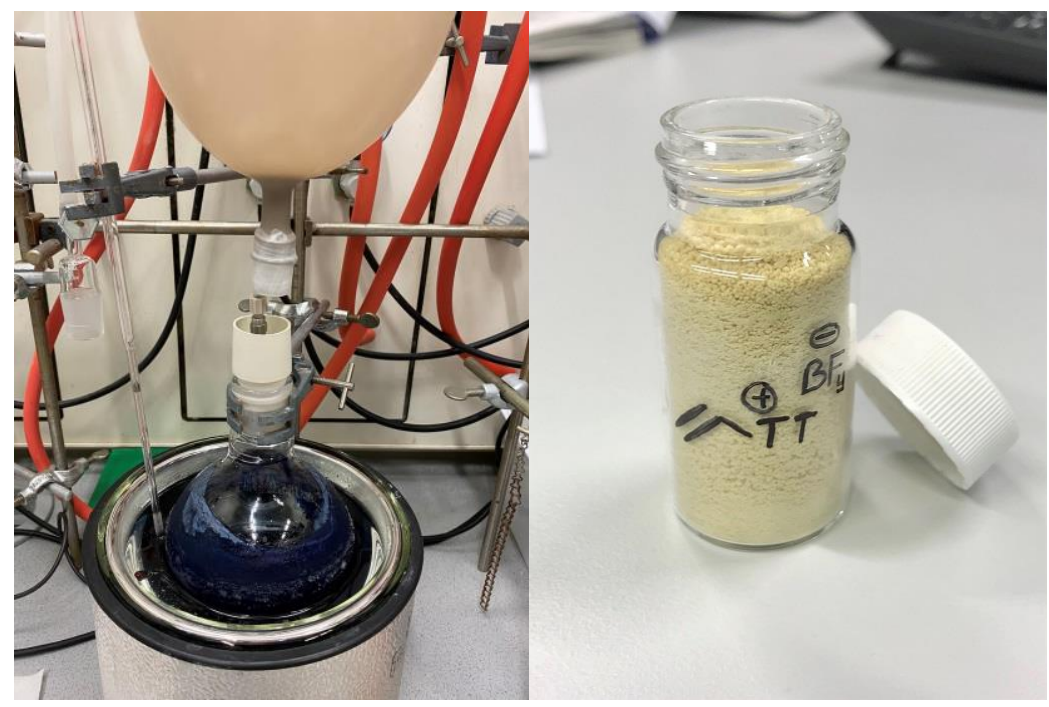


Figure S1. Pictures of the reaction setup (left) and the isolated product (right) on the preparation of 1.

Preparation of 1 from vinyltrimethylsilane

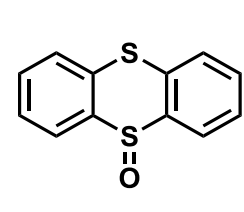

2

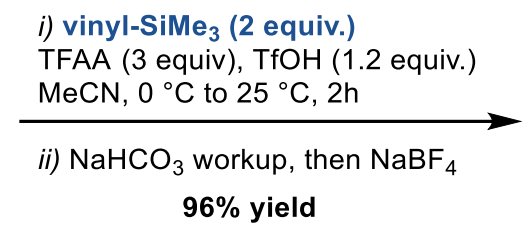

$96 \%$ yield

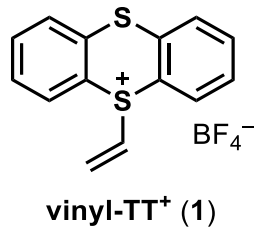

vinyl-TT+ $(1)$

To a round-bottom flask equipped with a stirring bar were added thianthrene-S-oxide 2 (3.14 g, $13.5 \mathrm{mmol}$, 1.00 equiv.), $65 \mathrm{~mL}$ of $\mathrm{MeCN}$ (c=0.20 M) and vinyl-SiMe $(4.0 \mathrm{~mL}, 2.7 \mathrm{~g}, 27 \mathrm{mmol}, 2.0$ equiv.). The solution was cooled down to $0{ }^{\circ} \mathrm{C}$ and trifluoroacetic anhydride $(5.7 \mathrm{~mL}, 8.6 \mathrm{~g}, 41 \mathrm{mmol}, 3.0$ equiv.), and trifluoromethane sulfonic acid (1.4 mL, $2.4 \mathrm{~g}, 16 \mathrm{mmol}, 1.2$ equiv.) were subsequently added. The resultant mixture was stirred at $0{ }^{\circ} \mathrm{C}$ for 30 minutes, warmed up to $25^{\circ} \mathrm{C}$ and stirred for additional 60 minutes. The mixture was concentrated under reduced pressure, diluted with DCM $(200 \mathrm{~mL})$, and washed with sat. aqueous $\mathrm{NaHCO}_{3}(200 \mathrm{~mL})$. The aqueous layer was extracted with $\mathrm{DCM}(2 \times 50 \mathrm{~mL})$. All organic phases were combined, washed with aqueous solutions of $5 \% \mathrm{NaBF}_{4}(2 \times 100 \mathrm{~mL})$, dried over $\mathrm{MgSO}_{4}$, filtered, and the solvent evaporated under reduced pressure. The crude material was dissolved in DCM (15 mL), and $\mathrm{Et}_{2} \mathrm{O}$ $(100 \mathrm{~mL})$ was subsequently added while stirring, causing the precipitation of a solid that was collected by filtration, washed with $\mathrm{Et}_{2} \mathrm{O}(3 \times 10 \mathrm{~mL})$, and dried under vacuum to afford 1 as an off-white solid $(4.30 \mathrm{~g}$, $13.0 \mathrm{mmol}, 96 \%)$.

\section{NMR Spectroscopy:}

${ }^{1} \mathrm{H}$ NMR $\left(500 \mathrm{MHz}, \mathrm{CD}_{3} \mathrm{CN}, 298 \mathrm{~K}, \delta\right): 8.21$ (dd, $\left.J=7.9,1.3 \mathrm{~Hz}, 2 \mathrm{H}\right), 8.00(\mathrm{dd}, J=7.9,1.3 \mathrm{~Hz}, 2 \mathrm{H}), 7.88$ (td, $J=7.8,1.4 \mathrm{~Hz}, 2 \mathrm{H}$ ), 7.77 (td, $J=7.8,1.4 \mathrm{~Hz}, 2 \mathrm{H}$ ), 6.65 (dd, $J=15.9,8.9 \mathrm{~Hz}, 1 \mathrm{H}$ ), 6.32 (dd, $J=8.9$, $3.1 \mathrm{~Hz}, 1 \mathrm{H}), 5.97(\mathrm{dd}, J=16.0,3.1 \mathrm{~Hz}, 1 \mathrm{H})$.

${ }^{13} \mathrm{C}$ NMR $\left(75 \mathrm{MHz}, \mathrm{CD}_{3} \mathrm{CN}, 298 \mathrm{~K}, \delta\right): 137.1,135.9,135.1,134.3,131.4,130.9,120.9,119.1$.

${ }^{19} \mathrm{~F}$ NMR $\left(282 \mathrm{MHz}, \mathrm{CD}_{3} \mathrm{CN}, 298 \mathrm{~K}, \delta\right):-151.38(\mathrm{~s}),-151.43$ (s).

HRMS-ESI (m/z) calculated for $\mathrm{C}_{14} \mathrm{H}_{11} \mathrm{~S}_{2}{ }^{+}$[M-BF $]^{+}$, 243.0297; found, 243.0298; deviation: -0.0 ppm.

IR (neat, thin film): $V_{\max }\left(\mathrm{cm}^{-1}\right)=3086,2360,2341,1568,1450,1435,1385,1288,1267,1033,1032,959$, $762,521,459$.

Melting point: $108-110^{\circ} \mathrm{C}$ (recryst. solvents: $\mathrm{DCM} / \mathrm{Et}_{2} \mathrm{O}$ )

Elemental analysis calcd (\%) for $\mathrm{C}_{14} \mathrm{H}_{11} \mathrm{BF}_{4} \mathrm{~S}_{2}$ : C 50.93, H 3.36, S 19.42; found: C 50.83, H 3.37, S 19.39. No decomposition was observed after storing vinyl-TT+ (1) between $20-28{ }^{\circ} \mathrm{C}$ under air. In Figure S2 is shown the ${ }^{1} \mathrm{H}$ NMR spectrum recorded for one sample kept in a vial with no further precautions for one year: 


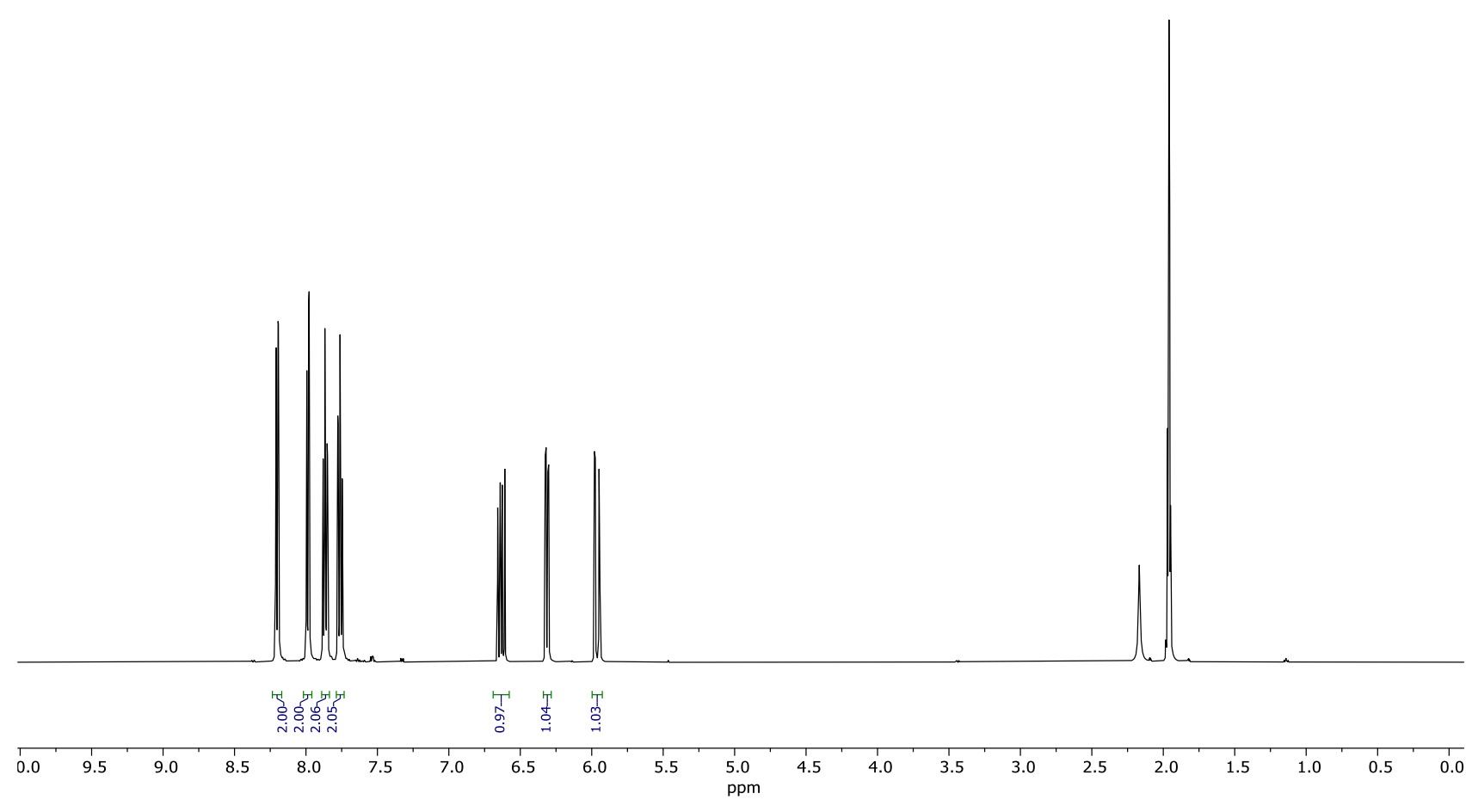

Figure S2. ${ }^{1} \mathrm{H}$ NMR spectrum of a sample of vinyl-TT+ (1) stored for one year, $\mathrm{CD}_{3} \mathrm{CN}, 500 \mathrm{MHz}, 298 \mathrm{~K}$.

In addition, no decomposition was observed after heating a solid sample of 1 up to $200{ }^{\circ} \mathrm{C}$ for 5 minutes. In Figure $\mathrm{S} 3$ is shown the ${ }^{1} \mathrm{H}$ NMR spectrum for one sample after heating.

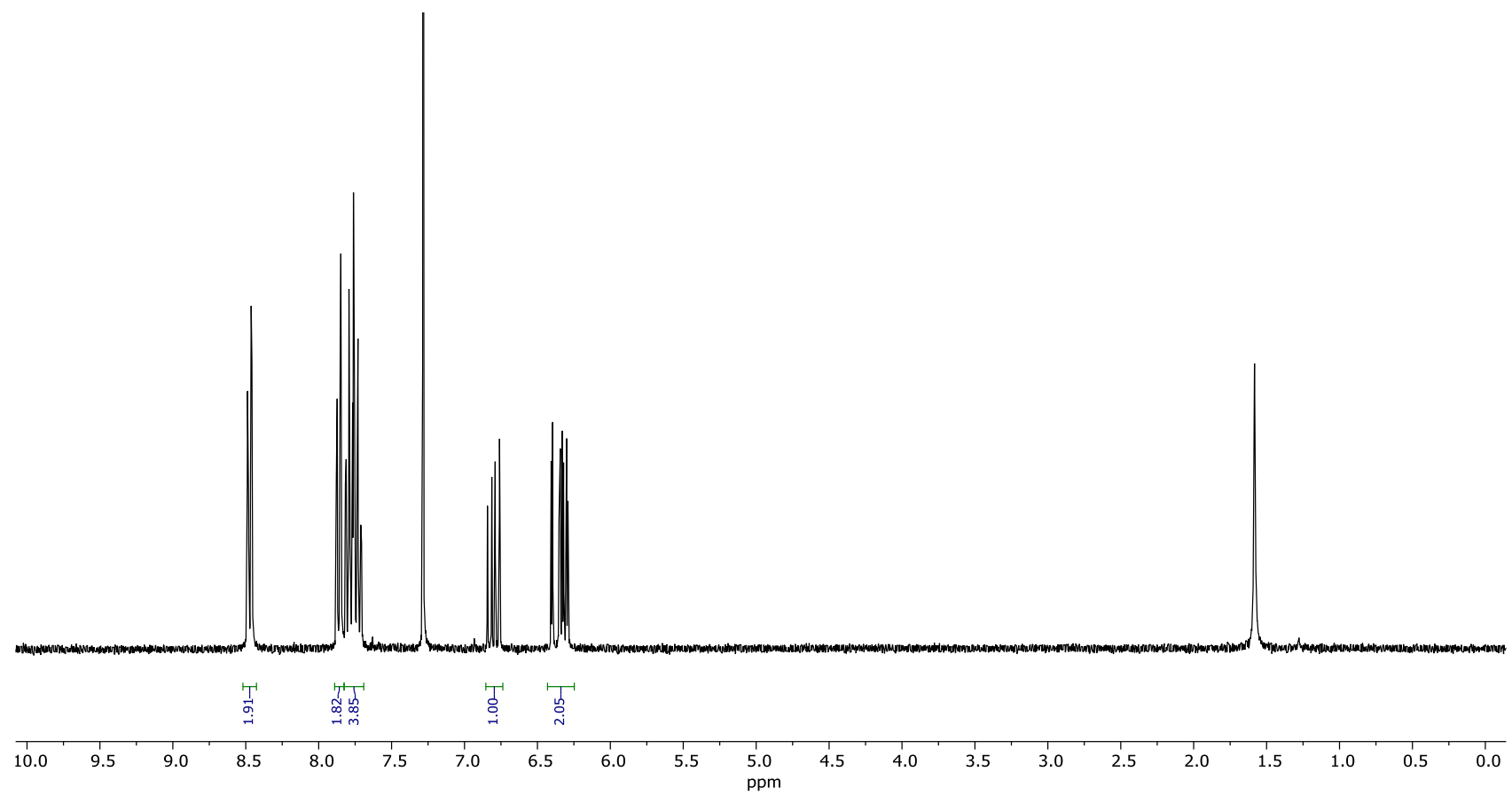

Figure S3. ${ }^{1} \mathrm{H}$ NMR spectrum of vinyl-TT+ (1) after heating up to $200{ }^{\circ} \mathrm{C}, \mathrm{CDCl}_{3}, 300 \mathrm{MHz}, 298 \mathrm{~K}$. 
UV-vis absorption spectra were recorded on a UV-2600 Shimadzu spectrophotometer using a $5 \times 10^{-5} \mathrm{M}$ solution of vinyl-TT+ (1) in MeCN.

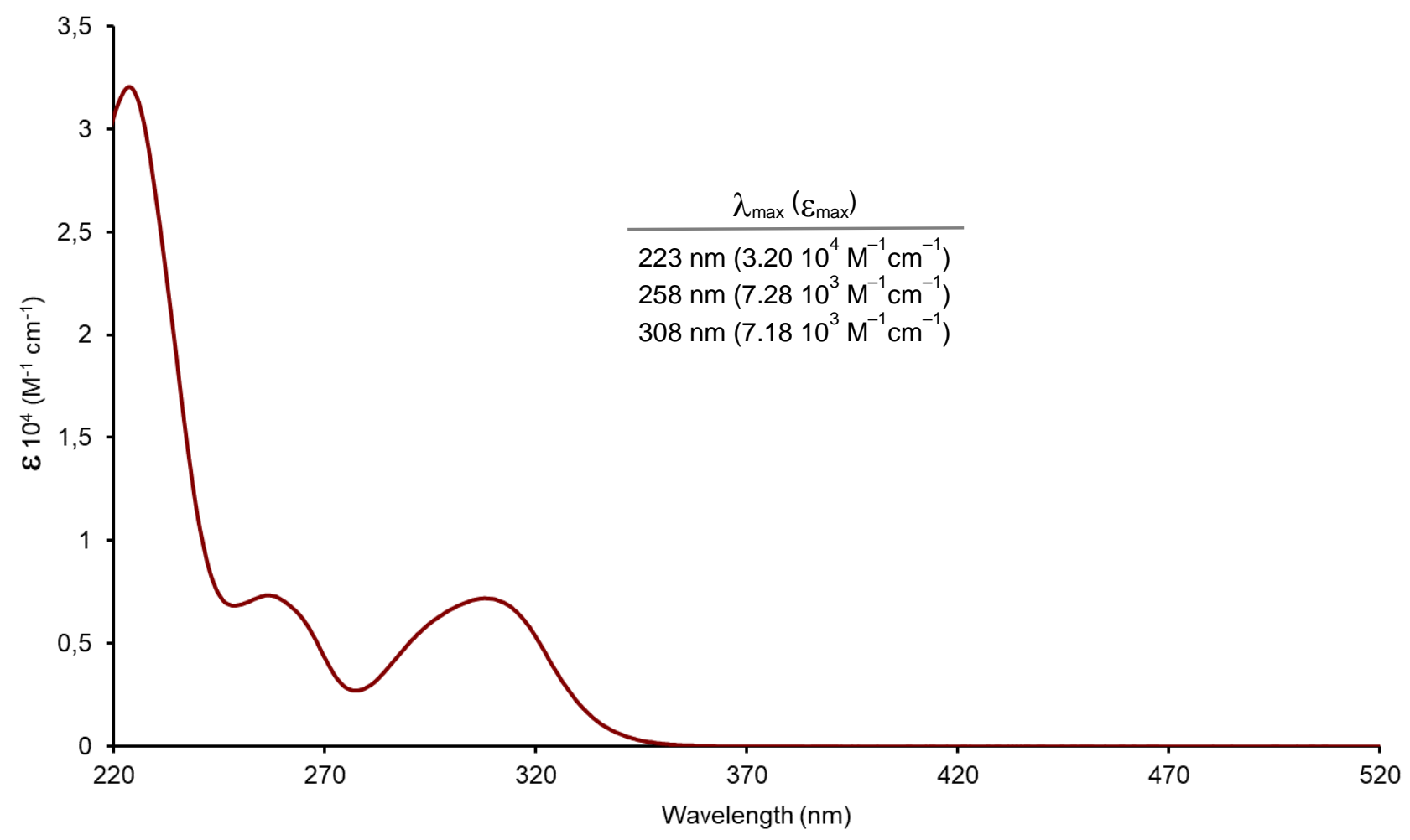

Figure S4. UV-vis absorption spectrum of 1 in solution $\left(5 \times 10^{-5} \mathrm{M}\right.$ in $\left.\mathrm{CH}_{3} \mathrm{CN}, 298 \mathrm{~K}\right)$.

\section{Cyclic voltammetry}

Cyclic voltammetry was conducted using an Autolab PGSTAT204 potentiostat equipped with a glassy carbon working electrode, a $\mathrm{Ag} / \mathrm{AgCl}$ reference electrode and a Pt counter electrode. Samples containing 10mM of vinyITT and $0.1 \mathrm{M}$ of tetrabutylammonium hexafluorophosphate were prepared in dry acetonitrile and degassed before measurement. Voltammograms were measured at $0.1 \mathrm{~V} / \mathrm{s}$ scan rate. Potentials values were converted to SCE substracting $0.047 \mathrm{~V}$ according to tabulated conversions. Potentials for irreversible waves were estimated at half the maximum current $\left(E_{\mathrm{p} / 2}\right)$, as previously described by Nicewicz. ${ }^{[8]}$ 


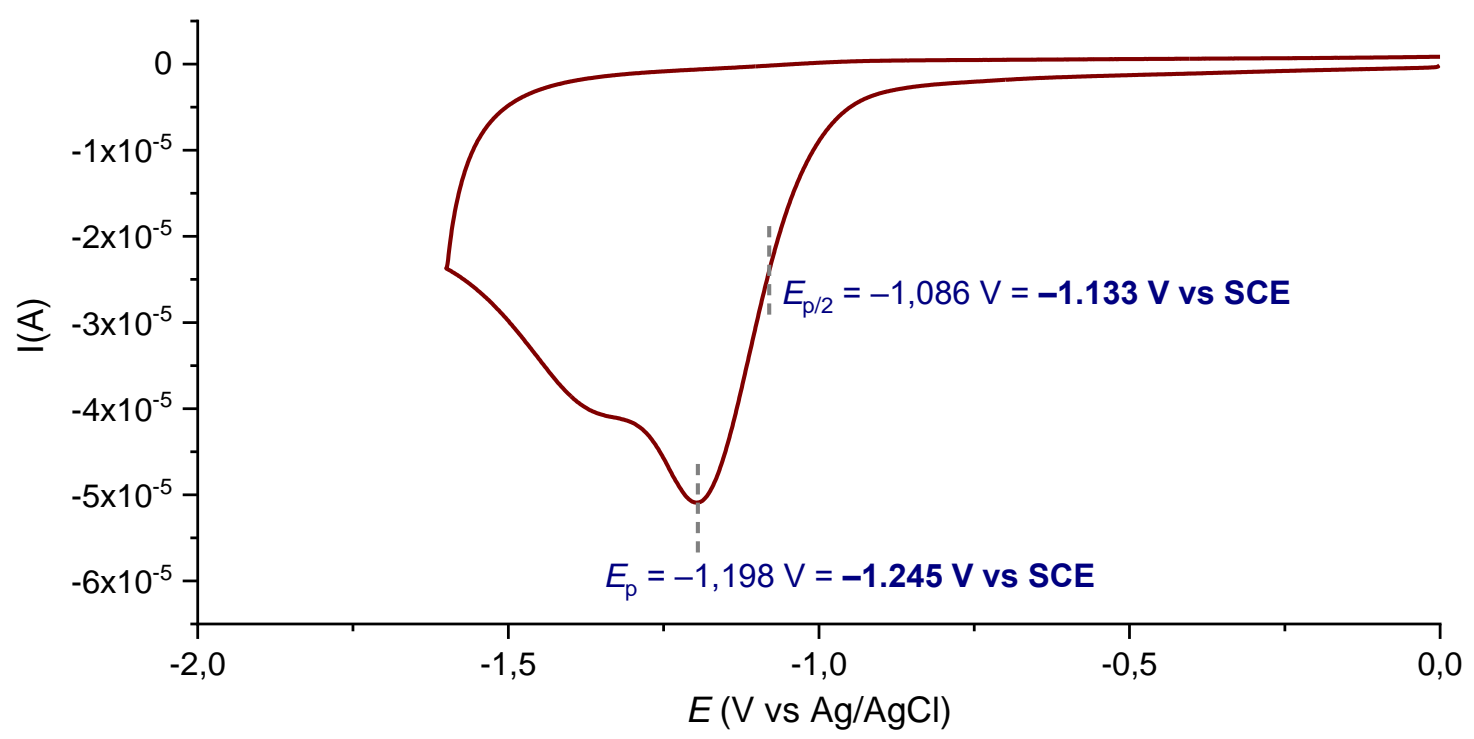

Figure S5. Cyclic voltammogram of 1.

\section{DSC-TGA analysis}

A blank group and an experimental group were tested under Ar purge. The whole process included heating and cooling regions. The blank group and measurement groups used aluminum crucibles with pierced lids (open crucibles) as a container.

Ar gas flow velocity: $50 \mathrm{~mL} / \mathrm{min}$

Heating region: from $25^{\circ} \mathrm{C}$ to $300 \stackrel{\circ}{\mathrm{C}}$

Heating rate: $3 \mathrm{~K} / \mathrm{min}$

Cooling region: from $300 \stackrel{\circ}{\circ}$ to $25 \stackrel{\circ}{\circ}$

Vinyl-TT+BF $4^{-}(\mathbf{1}$, powder, $10.4566 \mathrm{mg}, 31.6707 \mu \mathrm{mol})$ was placed in an Al-crucible for the DSC-TGA measurement. At $108^{\circ} \mathrm{C}$, melting of the reagent begins, which corresponds to the first endothermic peak in the DSC curve (Figure S6, bottom). Decomposition of 1 begins at around $280^{\circ} \mathrm{C}$, which is an exothermic process. 


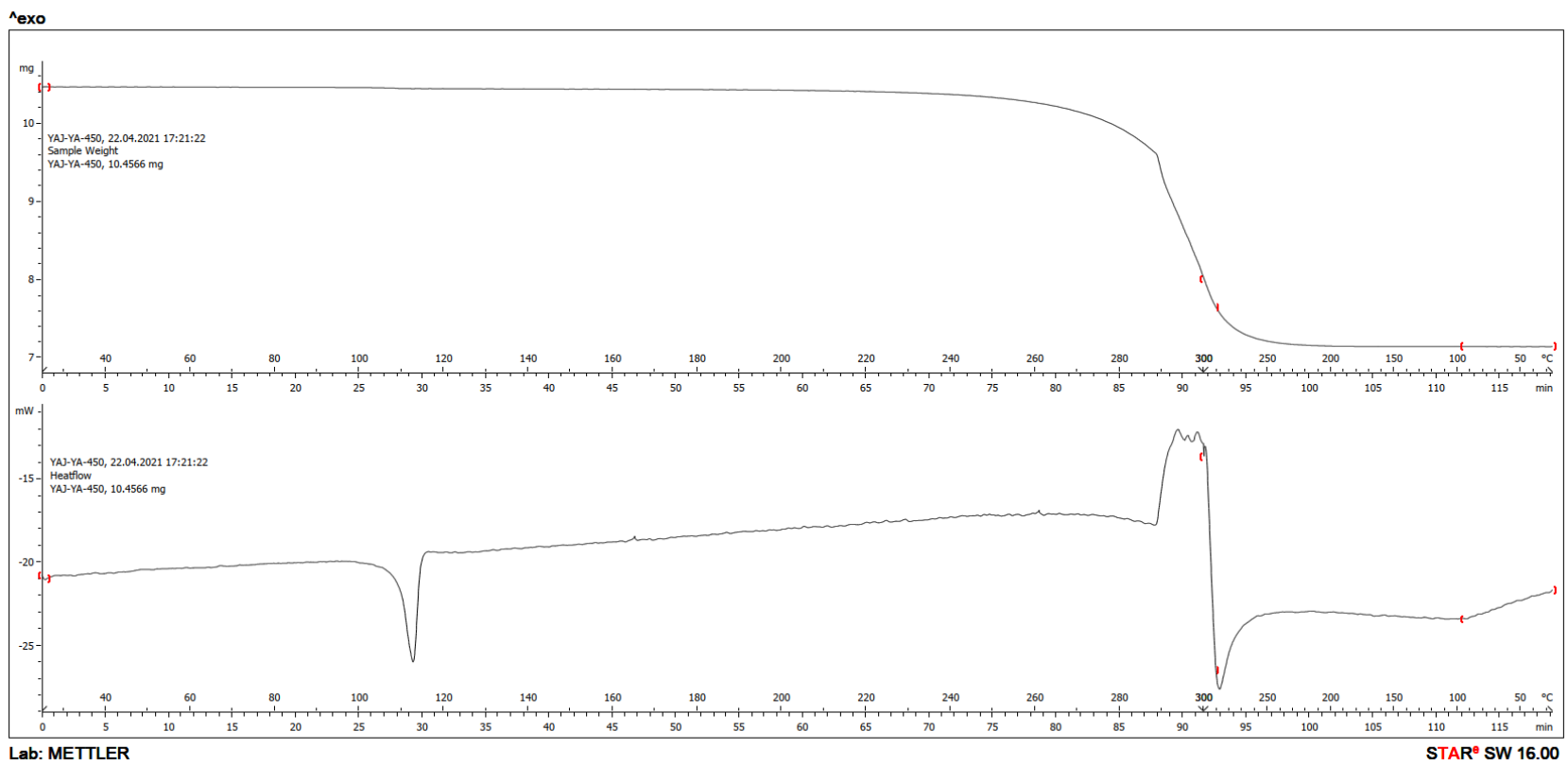

Figure S6. DSC-TGA analysis of 1.

Preparation of $1-d_{3}$ from ethylene- $d_{4}$<smiles>O=S1c2ccccc2Sc2ccccc21</smiles>

2

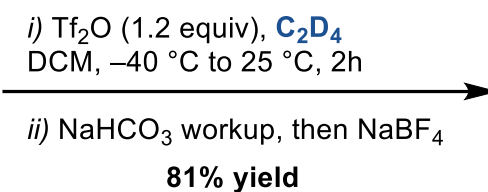

$81 \%$ yield

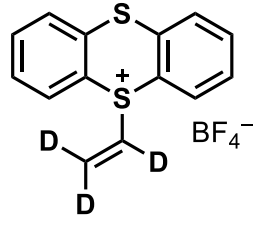

vinyl-TT $-d_{3}\left(1-d_{3}\right)$

To a $100 \mathrm{~mL}$ round-bottom flask equipped with a stirring bar was added thianthrene-S-oxide 2 (1.16 g,

$5.00 \mathrm{mmol}, 1.00$ equiv.). The flask was capped with a rubber septum and was evacuated. The flask was then cooled down to $-40^{\circ} \mathrm{C}$ and was backfilled with ethylene- $d_{4}$. DCM $(50 \mathrm{~mL}, \mathrm{c}=0.10 \mathrm{M})$ was added into the flask via a syringe and the solution was stirred at $-40^{\circ} \mathrm{C}$ for $10 \mathrm{~min}$. A small balloon filled with argon was then attached to the flask through a needle to balance pressure. Triflic anhydride $(1.01 \mathrm{~mL}, 1.69 \mathrm{~g}, 6.00 \mathrm{mmol}$, 1.20 equiv.) was added dropwise to the reaction at $-40^{\circ} \mathrm{C}$, and a dark purple suspension was formed progressively. After 30 minutes, the cooling bath was removed and the mixture was stirred for 1.5 hour at $25^{\circ} \mathrm{C}$. The balloon and the rubber septum were removed, and sat. aqueous $\mathrm{NaHCO}_{3}(30 \mathrm{~mL})$ was added carefully. The mixture was poured into a separation funnel and was vigorously shaken. The phases were separated and the aqueous layer was extracted with DCM $(2 \times 20 \mathrm{~mL})$. The combined organic phase was washed with aqueous of $5 \% \mathrm{NaBF}_{4}(4 \times 60 \mathrm{~mL})$, dried over $\mathrm{MgSO}_{4}$, filtered and evaporated under reduced pressure. The crude material was dissolved in DCM $(5 \mathrm{~mL})$, and $\mathrm{Et}_{2} \mathrm{O}(50 \mathrm{~mL})$ was subsequently added while stirring at $-40^{\circ} \mathrm{C}$, causing the precipitation of a solid that was collected by filtration, washed with $\mathrm{Et}_{2} \mathrm{O}$ $(3 \times 10 \mathrm{~mL})$, and dried under vacuum to afford $1-d_{3}$ as an off-white solid (1.35 g, $4.06 \mathrm{mmol}, 81 \%$ yield).

Deuterium incorporation: $>2.99{ }^{2} \mathrm{H} /$ molecule $\left({ }^{1} \mathrm{H}\right.$ NMR) 


\section{NMR Spectroscopy:}

${ }^{1} \mathrm{H}$ NMR (500 MHz, $\left.\mathrm{CD}_{2} \mathrm{Cl}_{2}, 298 \mathrm{~K}, \delta\right): 8.34-8.21(\mathrm{~m}, 2 \mathrm{H}), 7.97-7.87(\mathrm{~m}, 2 \mathrm{H}), 7.85-7.78(\mathrm{~m}, 2 \mathrm{H})$, 7.73 (ddd, $J=7.9,7.4,1.4 \mathrm{~Hz}, 2 \mathrm{H}$ ).

${ }^{2} \mathrm{H}$ NMR (92 MHz, $\left.\mathrm{CD}_{2} \mathrm{Cl}_{2}, 298 \mathrm{~K}, \delta\right): 6.74(\mathrm{~s}, 1 \mathrm{H}), 6.35(\mathrm{~s}, 1 \mathrm{H}), 6.22(\mathrm{~s}, 1 \mathrm{H})$.

${ }^{13} \mathrm{C}$ NMR (151 MHz, $\left.\mathrm{CD}_{2} \mathrm{Cl}_{2}, 298 \mathrm{~K}, \delta\right): 136.7,135.3,134.5,134.2(\mathrm{~m}), 130.7,130.6,119.3$ (t, $J=$ $30.5 \mathrm{~Hz}), 118.6$.

${ }^{19}$ F NMR (470 MHz, $\left.\mathrm{CD}_{2} \mathrm{Cl}_{2}, 298 \mathrm{~K}, \delta\right):-151.81(\mathrm{~s}),-151.87$ (s).

HRMS-ESI (m/z) calculated for $\left.\mathrm{C}_{14} \mathrm{H}_{8} \mathrm{D}_{3} \mathrm{~S}_{2}{ }^{+}[\mathrm{M}-\mathrm{BF}]_{4}\right]^{+}, 246.0486$; found, 246.0485; deviation: $-0.5 \mathrm{ppm}$.

\section{Preparation of [4+2] cycloadduct intermediate 3}<smiles>O=S1c2ccccc2Sc2ccccc21</smiles>

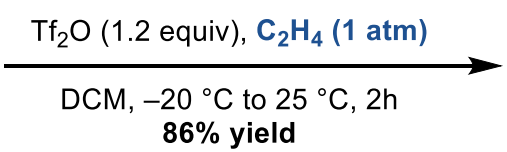

$86 \%$ yield

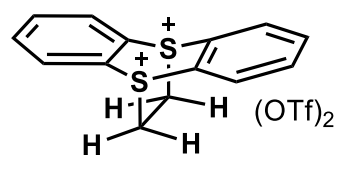

3

To a round-bottom flask equipped with a stirring bar were added thianthrene-S-oxide 2 (232 mg, $1.00 \mathrm{mmol}$, 1.00 equiv.) and $8.0 \mathrm{~mL}$ of $\mathrm{DCM}(\mathrm{C}=0.13 \mathrm{M})$. The flask was capped with a rubber septum and cooled down to $-40^{\circ} \mathrm{C}$. Through the solution was then bubbled ethylene gas for 5 minutes, after which a balloon filled with ethylene was connected to the flask to maintain the ethylene atmosphere throughout the reaction. Triflic anhydride (202 $\mu \mathrm{L}, 338 \mathrm{mg}, 1.20 \mathrm{mmol}, 1.20$ equiv.) was added dropwise to the reaction and a dark purple suspension was progressively formed. After 20 minutes the cooling bath was removed, and the mixture was stirred for 1.5 hour at $25^{\circ} \mathrm{C}$. The ethylene balloon and the rubber septum were removed, and $\mathrm{Et}_{2} \mathrm{O}(20 \mathrm{~mL})$ was added. The resultant precipitate was collected by filtration, washed with $\mathrm{Et}_{2} \mathrm{O}(3 \times 5 \mathrm{~mL})$, and dried under vacuum to give 3 as a colourless powder ( $467 \mathrm{mg}, 0.861 \mathrm{mmol}, 86 \%)$.

\section{NMR Spectroscopy:}

${ }^{1} \mathrm{H}$ NMR (500 MHz, CD $\left.{ }_{3} \mathrm{CN}, 298 \mathrm{~K}, \delta\right): \delta 8.56-8.50(\mathrm{~m}, 4 \mathrm{H}), 8.14-8.09(\mathrm{~m}, 4 \mathrm{H}), 4.18(\mathrm{~s}, 4 \mathrm{H})$.

${ }^{13} \mathrm{C}$ NMR $\left(75 \mathrm{MHz}, \mathrm{CD}_{3} \mathrm{CN}, 298 \mathrm{~K}, \delta\right): 136.9,136.4,126.3,37.6$.

HRMS-ESI (m/z) calculated for $\mathrm{C}_{14} \mathrm{H}_{12} \mathrm{~S}_{2}{ }^{2+}[\mathrm{M}-2 \mathrm{OTf}]^{2+}$, 122.0185; found, 122.0185; deviation: -0.4 ppm.

\section{Reaction of other sulfoxides with ethylene}

Reaction of diphenylsulfoxide with ethylene<smiles>O=S(c1ccccc1)c1ccccc1</smiles>

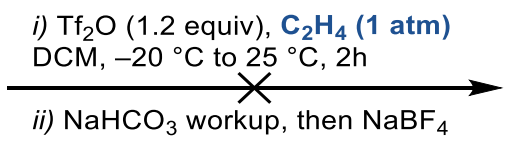<smiles>C=C[S+](c1ccccc1)c1ccccc1</smiles>

To a round-bottom flask equipped with a stirring bar were added diphenylsulfoxide $(101 \mathrm{mg}, 0.500 \mathrm{mmol}$, 
1.00 equiv.) and $4 \mathrm{~mL}$ of $\mathrm{DCM}(\mathrm{c}=0.125 \mathrm{M})$. The flask was capped with a rubber septum and cooled down to $-40^{\circ} \mathrm{C}$. Through the solution was then bubbled ethylene gas for 5 minutes, after which a balloon filled with ethylene was connected to the flask to maintain the ethylene atmosphere throughout the reaction. Triflic anhydride (101 $\mathrm{L}, 169 \mathrm{mg}, 0.600 \mathrm{mmol}, 1.20$ equiv.) was added dropwise to the reaction. After 20 minutes the cooling bath was removed and the mixture was stirred for 1.5 hour at $25^{\circ} \mathrm{C}$. The ethylene balloon and the rubber septum were removed, and solution was concentrated under reduced pressure, diluted with DCM (20 $\mathrm{mL})$ and washed with sat. aqueous $\mathrm{NaHCO}_{3}(20 \mathrm{~mL})$. The aqueous layer was extracted with $\mathrm{DCM}(2 \times 10$ $\mathrm{mL})$. All organic phases were combined, washed with aqueous solutions of $5 \% \mathrm{NaBF}_{4}(2 \times 10 \mathrm{~mL})$, dried over $\mathrm{MgSO}_{4}$, filtered, and the solvent evaporated to dryness under reduced pressure. $\mathrm{CDCl}_{3}(1 \mathrm{~mL})$ was then added and the crude was analyzed by ${ }^{1} \mathrm{H}$ NMR, but no vinyl-SPh${ }_{2}{ }^{+}$could be detected.

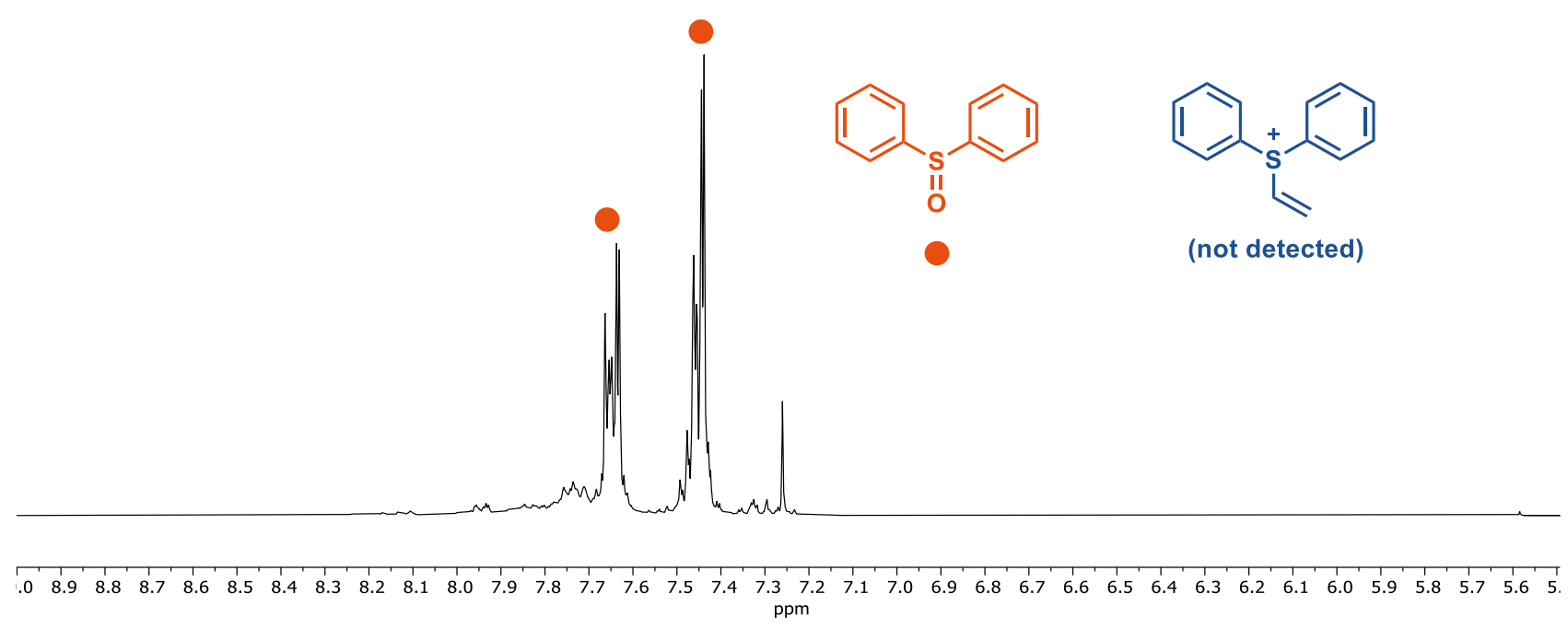

Figure S7. ${ }^{1} \mathrm{H}$ NMR spectrum of the crude of the reaction of diphenylsulfoxide and $\mathrm{Tf}_{2} \mathrm{O}$ under ethylene atmosphere, $\mathrm{CDCl}_{3}, 300 \mathrm{MHz}, 298 \mathrm{~K}$.

Reaction of dibenzothiophene-S-oxide with ethylene
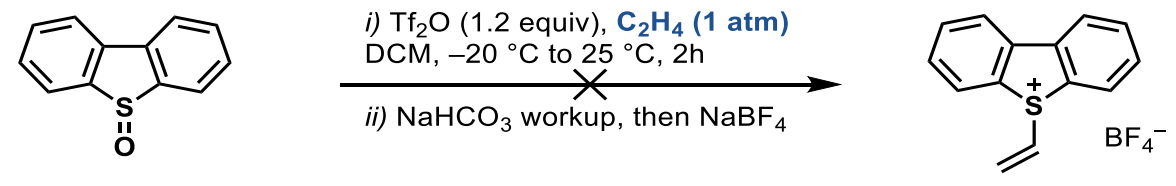

To a round-bottom flask equipped with a stirring bar were added dibenzothiophene-S-oxide (100 mg, $0.500 \mathrm{mmol}, 1.00$ equiv.) and $4 \mathrm{~mL}$ of $\mathrm{DCM}(\mathrm{c}=0.125 \mathrm{M})$. The flask was capped with a rubber septum and cooled down to $-40^{\circ} \mathrm{C}$. Through the solution was then bubbled ethylene gas for 5 minutes, after which a balloon filled with ethylene was connected to the flask to maintain the ethylene atmosphere throughout the reaction. Triflic anhydride (101 $\mu \mathrm{L}, 169 \mathrm{mg}, 0.600 \mathrm{mmol}, 1.20$ equiv.) was added dropwise to the reaction. After 20 minutes the cooling bath was removed and the mixture was stirred for 1.5 hour at $25^{\circ} \mathrm{C}$. The ethylene balloon and the rubber septum were removed, and solution was concentrated under reduced pressure, diluted with $\mathrm{DCM}(20 \mathrm{~mL})$ and washed with aqueous $\mathrm{NaHCO}_{3}(20 \mathrm{~mL})$. The aqueous layer was extracted with DCM $(2 \times 10 \mathrm{~mL})$. All organic phases were combined, washed with aqueous solutions of $5 \%$ 
$\mathrm{NaBF}_{4}(2 \times 10 \mathrm{~mL})$, dried over $\mathrm{MgSO}_{4}$, filtered, and the solvent evaporated to dryness under reduced pressure. $\mathrm{CDCl}_{3}(1 \mathrm{~mL})$ was then added and the crude was analyzed by ${ }^{1} \mathrm{H} \mathrm{NMR}$, but no $S$-vinyl-sulfonium salt could be detected.

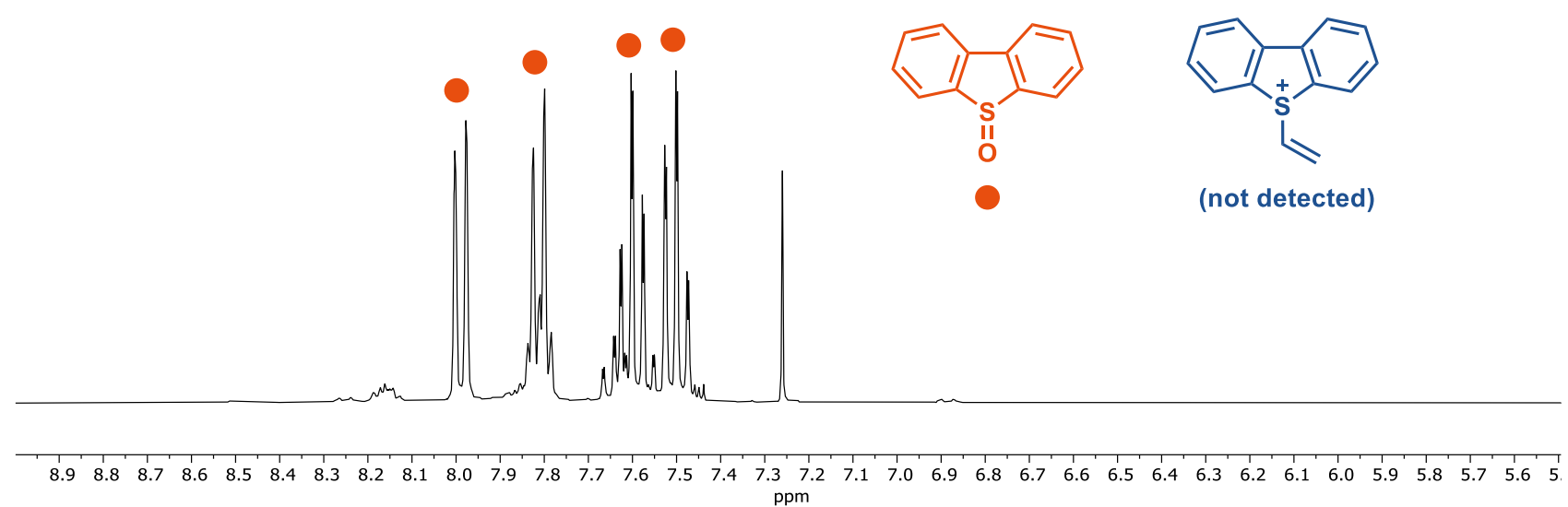

Figure S8. ${ }^{1} \mathrm{H}$ NMR spectrum of the crude of the reaction of dibenzothiophene-S-oxide and $\mathrm{Tf}_{2} \mathrm{O}$ under ethylene atmosphere, $\mathrm{CDCl}_{3}, 300 \mathrm{MHz}, 298 \mathrm{~K}$.

\section{Preparation of vinyl-SPh 2 (OTf) (S3)}

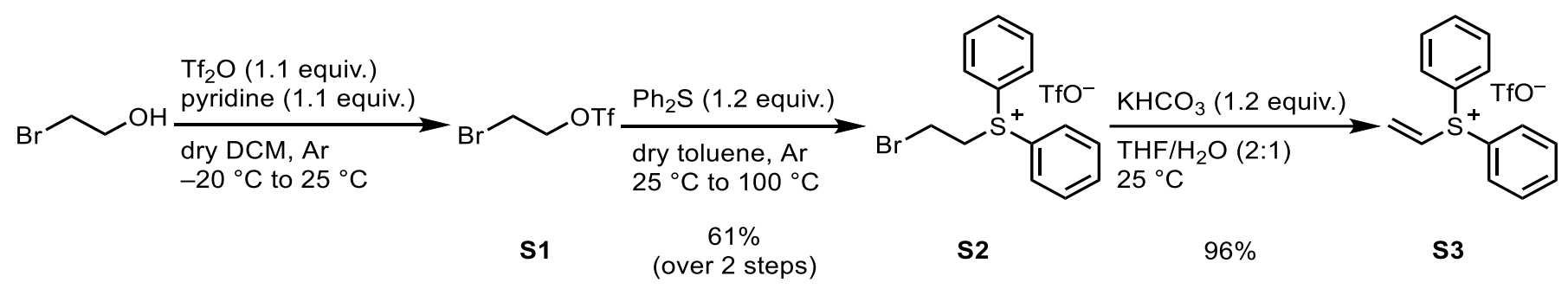

This compound was prepared following the 3-step procedure reported by Aggarwal:[2]

To an oven-dried $100 \mathrm{~mL}$ round-bottom flask under argon atmosphere containing a teflon-coated magnetic stirring bar were added pyridine (1.7 g, $1.7 \mathrm{~mL}, 22 \mathrm{mmol}, 1.1$ equiv.) and anhydrous $\mathrm{DCM}(35 \mathrm{~mL})$ and the mixture was cooled down to $-20^{\circ} \mathrm{C}$. Trifluoromethanesulfonic anhydride $(5.9 \mathrm{~g}, 3.5 \mathrm{~mL}, 21 \mathrm{mmol}, 1.1$ equiv.) was added dropwise and the reaction mixture was allowed to stir for 10 minutes at the same temperature. 2Bromoethanol ( $2.5 \mathrm{~g}, 1.4 \mathrm{~mL}, 20 \mathrm{mmol}, 1.0$ equiv.) was then added dropwise to the reaction mixture under $20{ }^{\circ} \mathrm{C}$. The cooling bath was removed and the reaction stirred for a further 10 minutes (do not allow more than this time) while warming. The resulting suspension was filtered, concentrated (using a rotary evaporator, keeping the water bath temp below $20^{\circ} \mathrm{C}$ ) and pentane $(30 \mathrm{~mL})$ was added. The mixture was filtered and the filtrate was concentrated again under reduced pressure and dried under vacuum to give the title product $\mathbf{S 1}$ as a clear colorless oil $(4.6 \mathrm{~g}, 89 \%)$. It was used immediately in the next step without further purification.

To a round-bottom flask under argon atmosphere containing a teflon-coated magnetic stirring bar were added S1 (4.58 g, $17.8 \mathrm{mmol}, 1.00$ equiv.), anhydrous toluene (20 mL) and diphenyl sulfide (4.0 g, $3.6 \mathrm{~mL}, 15 \mathrm{mmol}$, 
1.2 equiv.) at $25^{\circ} \mathrm{C}$. The reaction mixture was then heated at $100^{\circ} \mathrm{C}$ under argon for $5 \mathrm{~h}$. The solution was allowed to cool to $25^{\circ} \mathrm{C}$ and diethyl ether $(20 \mathrm{~mL})$ was added. The resulting mixture was filtered and the residue was washed with diethyl ether $(10 \mathrm{~mL})$ to afford $5.4 \mathrm{~g}$ of the title compound $\mathbf{S 2}$ (69\% yield) as a white power.

Under ambient atmosphere, a $20 \mathrm{~mL}$ vail equipped with a teflon-coated magnetic stirring bar was charged with S2 (443 mg, $1.00 \mathrm{mmol}, 1.00$ equiv.) and $\mathrm{THF} / \mathrm{H}_{2} \mathrm{O}$ (2:1) (3 mL). $\mathrm{KHCO}_{3}$ (120 mg, $1.20 \mathrm{mmol}$, 1.20 equiv.) was added in one portion and the reaction mixture was stirred for $30 \mathrm{~min}$ at $25^{\circ} \mathrm{C}$ (do not allow more than this time). Water $(1 \mathrm{~mL})$ was added and the mixture was extracted with DCM $(3 \times 5 \mathrm{~mL})$. The organic layers were collected, dried over anhydrous $\mathrm{Na}_{2} \mathrm{SO}_{4}$, filtered and concentrated under reduced pressure. The residue was purified by column chromatography on silica gel eluting with a solvent mixture of methonal:DCM (1:15 (v:v)) to afford $348 \mathrm{mg}$ of the title compound (S3) as a yellow oil ( $96 \%$ yield). The NMR spectra are in accordance with the literature. ${ }^{[2]}$

\section{Annulation reactions employing vinyl-TT+ 1}

Spiro[cyclopropane-1,3'-indolin]-2'-one (5)
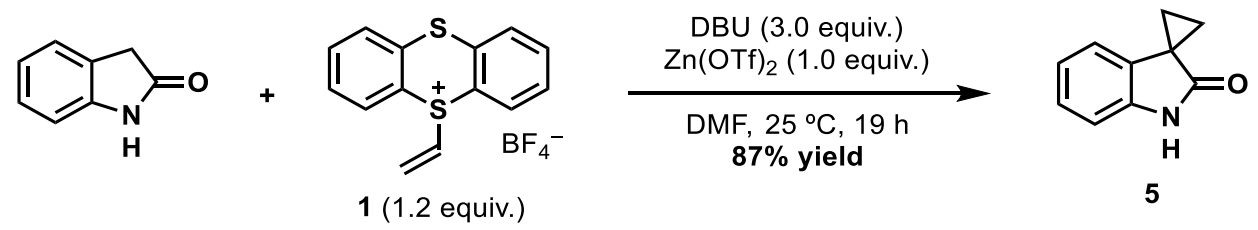

Following a modified reported procedure, ${ }^{[9]}$ 2-oxindole (26.6 mg, $0.200 \mathrm{mmol}, 1.00$ equiv.), 1 (79.2 mg, $0.240 \mathrm{mmol}, 1.20$ equiv.), and zinc triflate $(72.7 \mathrm{mg}, 0.200 \mathrm{mmol}, 1.00$ equiv.) were dissolved in DMF (1.0 mL, $C=0.20 \mathrm{M})$ under ambient atmosphere. To this solution was added DBU $(90 \mu \mathrm{L}, 92 \mathrm{mg}, 0.60 \mathrm{mmol}$, 3.0 equiv.). After stirring for $19 \mathrm{~h}$ at $25^{\circ} \mathrm{C}$, a saturated aqueous solution of $\mathrm{NH}_{4} \mathrm{Cl}(7 \mathrm{~mL})$ was added, and the phases were separated. The aqueous phase was extracted with EtOAc $(3 \times 50 \mathrm{~mL})$. All organic phases were combined, washed with water $(2 \times 10 \mathrm{~mL})$, dried over $\mathrm{Na}_{2} \mathrm{SO}_{4}$, filtered, and concentrated under reduced

pressure. Purification of the residue by column chromatography on silica gel eluting with hexanes/EtOAc (3:1, $\mathrm{v} / \mathrm{v}$ ) afforded the title compound as a pale-yellow solid (27.6 mg, $0.173 \mathrm{mmol}, 87 \%)$. The NMR spectra are in good accordance with the literature.[9]

$\mathbf{R} \boldsymbol{f}=0.40$ (hexanes/EtOAc, 1:1).

\section{NMR Spectroscopy:}

${ }^{1} \mathrm{H}$ NMR $\left(500 \mathrm{MHz}, \mathrm{CDCl}_{3}, 298 \mathrm{~K}, \delta\right): 9.43(\mathrm{~s}, 1 \mathrm{H}), 7.22-7.16(\mathrm{~m}, 1 \mathrm{H}), 7.04-6.97(\mathrm{~m}, 2 \mathrm{H}), 6.83(\mathrm{~m}, 1 \mathrm{H})$, $1.81-1.74(\mathrm{~m}, 2 \mathrm{H}), 1.58-1.51(\mathrm{~m}, 2 \mathrm{H})$.

${ }^{13} \mathrm{C}$ NMR $\left(126 \mathrm{MHz}, \mathrm{CDCl}_{3}, 298 \mathrm{~K}, \mathrm{\delta}\right): 180.0,141.0,131.4,126.9,122.1,118.7,110.1,27.7,19.6$.

HRMS-EI (m/z) calculated for $\mathrm{C}_{10} \mathrm{H}_{9} \mathrm{NO}^{+}[\mathrm{M}]^{+}, 159.0679$; found, 159.0680; deviation: -1.0 ppm. 
Methyl ( \pm )-4-tosylmorpholine-3-carboxylate (7)<smiles>[Y]N[C@@H](CO)C(C)=O</smiles><smiles>C=C[S+]1c2ccccc2Sc2ccccc21</smiles>

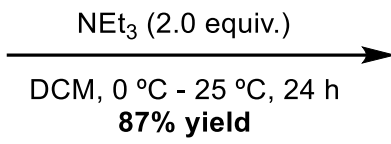

$87 \%$ yield

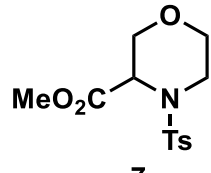

7

Following a modified reported procedure, ${ }^{[2]} \mathrm{N}$-tosyl DL-serine methyl ester (54.7 mg, $0.200 \mathrm{mmol}, 1.00$ equiv.) was dissolved in dry DCM $(1.0 \mathrm{~mL}, C=0.20 \mathrm{M})$ under an argon atmosphere, and the resulting solution was cooled to $0^{\circ} \mathrm{C}$. To the solution was added dry NEt3 $(56 \mu \mathrm{L}, 41 \mathrm{mg}, 0.40 \mathrm{mmol}, 2.0$ equiv.), and after $10 \mathrm{~min}$ a solution of 1 (87.4 mg, $0.265 \mathrm{mmol}, 1.32$ equiv.) in dry DCM $(0.5 \mathrm{~mL})$ was added dropwise. After stirring for $3 \mathrm{~h}$ at $0{ }^{\circ} \mathrm{C}$, and then for $21 \mathrm{~h}$ at $25^{\circ} \mathrm{C}$, a saturated aqueous solution of $\mathrm{NH}_{4} \mathrm{Cl}(3 \mathrm{~mL})$ was added. The phases were separated, and the aqueous phase was extracted with DCM $(3 \times 20 \mathrm{~mL})$. All organic phases were combined, washed with brine $(20 \mathrm{~mL})$, dried over $\mathrm{MgSO}_{4}$, filtered, and concentrated under reduced pressure. Purification of the residue by column chromatography on silica gel eluting with hexanes/EtOAc (3:1 to 1:1, $\mathrm{v} / \mathrm{v}$ ) afforded the title compound as a colorless solid (52.2 $\mathrm{mg}, 0.174 \mathrm{mmol}, 87 \%)$. The NMR spectra are in good accordance with the literature.[2]

$\mathbf{R}_{\boldsymbol{f}}=0.54$ (hexanes/EtOAc).

\section{NMR Spectroscopy:}

${ }^{1} \mathrm{H}$ NMR $\left(500 \mathrm{MHz}, \mathrm{CDCl}_{3}, 298 \mathrm{~K}, \delta\right): 7.65(\mathrm{~d}, J=8.3 \mathrm{~Hz}, 2 \mathrm{H}), 7.29(\mathrm{~d}, J=8.3 \mathrm{~Hz}, 2 \mathrm{H}), 4.50$ (d, $J=3.3 \mathrm{~Hz}, 1 \mathrm{H}), 4.26(\mathrm{~d}, J=11.6 \mathrm{~Hz}, 1 \mathrm{H}), 3.87-3.82(\mathrm{~m}, 1 \mathrm{H}), 3.69(\mathrm{dd}, J=11.6,3.7 \mathrm{~Hz}, 1 \mathrm{H}), 3.57-3.44$ $(\mathrm{m}, 6 \mathrm{H}), 2.42(\mathrm{~s}, 3 \mathrm{H})$.

${ }^{13} \mathrm{C}$ NMR $\left(126 \mathrm{MHz}, \mathrm{CDCl}_{3}, 298 \mathrm{~K}, \delta\right): 169.6,143.7,136.5,129.7,127.5,68.9,66.8,55.5,52.4,42.0$, 21.7.

HRMS- $\mathrm{Cl}(\mathbf{m} / \mathbf{z})$ calculated for $\mathrm{C}_{13} \mathrm{H}_{18} \mathrm{NO}_{5} \mathrm{~S}^{+}[\mathrm{M}+\mathrm{H}]^{+}$, 300.0900; found, 300.0901; deviation: -0.2 ppm.

Ethyl ( \pm )-2-phenyl-1-tosylazetidine-2-carboxylate (9)

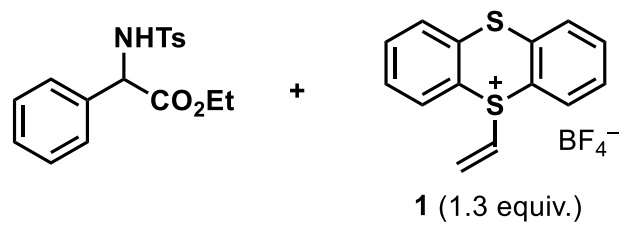

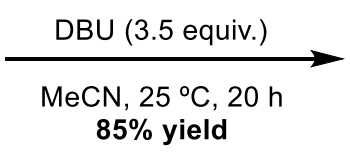

$85 \%$ yield

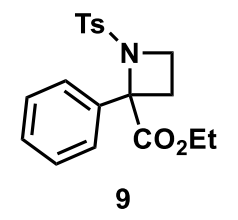

Following a modified reported procedure, ${ }^{[10]}$ a vial was charged with $N$-tosyl DL-phenylglycine ethyl ester (55.7 mg, $0.167 \mathrm{mmol}, 1.00$ equiv.), 1 (69.3 mg, $0.210 \mathrm{mmol}, 1.26$ equiv.), and $\mathrm{MeCN}$ (2.3 mL, $c=0.073 \mathrm{M}$ ) at $25^{\circ} \mathrm{C}$ under air. Then, DBU ( $87 \mu \mathrm{L}, 89 \mathrm{mg}, 0.58 \mathrm{mmol}, 3.5$ equiv.) was added. The reaction was stirred for $20 \mathrm{~h}$ at $25^{\circ} \mathrm{C}$ and was then concentrated under reduced pressure. The residue was purified by column chromatography on silica gel eluting with pentane/EtOAc (100\% pentane to $5: 1, \mathrm{v} / \mathrm{v})$. The title compound (51.2 $\mathrm{mg}, 0.142 \mathrm{mmol}, 85 \%)$ was obtained as a colorless oil. The NMR spectra are in good accordance with 
the literature. ${ }^{[10]}$

$\mathbf{R}_{\boldsymbol{f}}=0.21$ (pentane/EtOAc, 5:1).

\section{NMR Spectroscopy:}

${ }^{1} \mathrm{H}$ NMR $\left(500 \mathrm{MHz}, \mathrm{CDCl}_{3}, 298 \mathrm{~K}, \delta\right): 7.58(\mathrm{~d}, J=8.3 \mathrm{~Hz}, 2 \mathrm{H}), 7.45-7.40(\mathrm{~m}, 2 \mathrm{H}), 7.36-7.28(\mathrm{~m}, 3 \mathrm{H})$, $7.23(\mathrm{~d}, J=8.3 \mathrm{~Hz}, 2 \mathrm{H}), 4.27-4.16(\mathrm{~m}, 3 \mathrm{H}), 3.82$ (ddd, $J=9.1,6.7,4.9 \mathrm{~Hz}, 1 \mathrm{H}), 2.90$ (ddd, $J=11.3,9.2$, $4.9 \mathrm{~Hz}, 1 \mathrm{H}), 2.57$ (ddd, $J=11.2,9.1,7.0 \mathrm{~Hz}, 1 \mathrm{H}), 2.41$ (s, 3H), 1.27 (t, $J=7.1 \mathrm{~Hz}, 3 \mathrm{H}$ ).

${ }^{13} \mathrm{C}$ NMR (126 MHz, $\left.\mathrm{CDCl}_{3}, 298 \mathrm{~K}, \delta\right): 170.8,143.4,139.3,136.9,129.5,128.4,128.1,127.5,126.4$, $77.4,62.2,47.5,29.3,21.6,14.0$.

HRMS-ESI (m/z) calculated for $\mathrm{C}_{19} \mathrm{H}_{21} \mathrm{NO}_{4} \mathrm{SNa}^{+}[\mathrm{M}+\mathrm{Na}]^{+}$, 382.1084; found, 382.1086; deviation: -0.5 ppm.

(士)-3-Tosyl-7-oxa-3-azabicyclo[4.1.0]heptane (11)

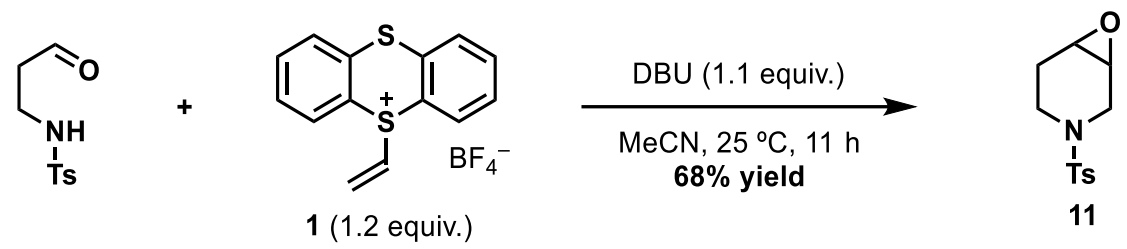

Epoxide 11 was prepared following a modified reported procedure. ${ }^{[11]}$ Under air, DBU $(14 \mu \mathrm{L}, 14 \mathrm{mg}$, $0.094 \mathrm{mmol}, 1.1$ equiv.) was added to a mixture of 4-methyl- $N$-(3-oxopropyl)benzenesulfonamide ${ }^{[4]}(19.0 \mathrm{mg}$, $0.0836 \mathrm{mmol}, 1.00$ equiv.) (used immediately after preparation) and $\mathbf{1}$ (33.1 $\mathrm{mg}, 0.100 \mathrm{mmol}, 1.20$ equiv.) in $\operatorname{MeCN}(1.0 \mathrm{~mL}, C=0.084 \mathrm{M})$. The reaction mixture was stirred at $25^{\circ} \mathrm{C}$ for $11 \mathrm{~h}$ and was then concentrated under reduced pressure. Purification of the residue by column chromatography on silica gel eluting with hexanes/EtOAc (3:1, v/v) afforded the title compound as a colorless solid (14.3 mg, $0.0565 \mathrm{mmol}, 68 \%)$. The NMR spectra are in good accordance with the literature. ${ }^{[11]}$

$\mathbf{R}_{\boldsymbol{f}}=0.48$ (hexanes/EtOAc, $1: 1$ ).

\section{NMR Spectroscopy:}

${ }^{1} \mathrm{H}$ NMR $\left(500 \mathrm{MHz}, \mathrm{CDCl}_{3}, 298 \mathrm{~K}, \delta\right): 7.62(\mathrm{~d}, J=8.2 \mathrm{~Hz}, 2 \mathrm{H}), 7.32(\mathrm{~d}, J=8.2 \mathrm{~Hz}, 2 \mathrm{H}), 3.85$ (ddd, $J=13.7,4.3,1.5 \mathrm{~Hz}, 1 \mathrm{H}), 3.41-3.33(\mathrm{~m}, 1 \mathrm{H}), 3.30-3.24(\mathrm{~m}, 2 \mathrm{H}), 3.09$ (d, $J=13.7 \mathrm{~Hz}, 1 \mathrm{H}), 2.56$ (ddd, $J=12.1,8.6,6.0 \mathrm{~Hz}, 1 \mathrm{H}), 2.43(\mathrm{~s}, 3 \mathrm{H}), 2.13-2.08(\mathrm{~m}, 2 \mathrm{H})$.

${ }^{13} \mathrm{C}$ NMR $\left(126 \mathrm{MHz}, \mathrm{CDCl}_{3}, 298 \mathrm{~K}, \delta\right): 143.9,133.4,129.9,127.7,50.5,49.9,44.3,39.3,25.4,21.7$. HRMS- $\mathrm{Cl}(\mathbf{m} / \mathbf{z})$ calculated for $\mathrm{C}_{12} \mathrm{H}_{16} \mathrm{~N}_{1} \mathrm{O}_{3} \mathrm{~S}_{1}{ }^{+}[\mathrm{M}+\mathrm{H}]^{+}$, 254.0845; found, 254.0844; deviation: 0.4 ppm. 


\section{Vinylation of $\mathbf{N}$-heterocycles using vinyl-TT+}

General procedure A
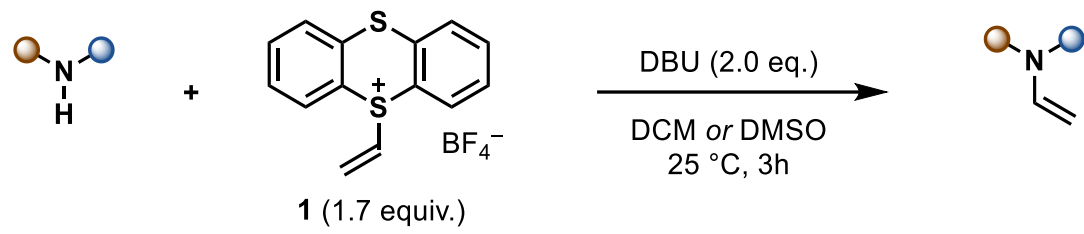

Under air, a vial was charged with the substrate to be vinylated $(0.300 \mathrm{mmol}, 1.00$ equiv.) and 1 (168 $\mathrm{mg}$, $0.510 \mathrm{mmol}, 1.70$ equiv.). DCM or DMSO (3.0 mL, $c=0.10 \mathrm{M}$ ) was added, followed by DBU (90 $\mu \mathrm{L}, 92 \mathrm{mg}$, $0.60 \mathrm{mmol}, 2.0$ equiv.), and the mixture was stirred at $25^{\circ} \mathrm{C}$ for $3 \mathrm{~h}$. Then, the solvent was removed under reduced pressure, and the residue was purified as indicated to give the corresponding product. Important note: some $N$-vinylated product were observed to decompose by polymerization during chromatography. To prevent this $1 \% \mathrm{NEt}_{3}$ was included in the eluent solvent system to deactivate silica.

9-Vinyl-9H-pyrido[3,4-b]indole (12)
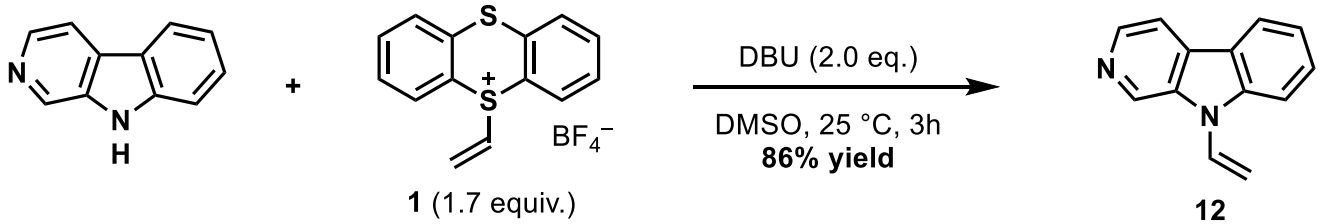

The title compound was prepared following general procedure A. Under air, a vial was charged with norharmane (50.5 mg, $0.300 \mathrm{mmol}, 1.00$ equiv.) and 1 (119 mg, $0.360 \mathrm{mmol}, 1.20$ equiv.). DMSO (3.0 mL, $C=0.10 \mathrm{M})$ was added, followed by DBU $(90 \mu \mathrm{L}, 92 \mathrm{mg}, 0.60 \mathrm{mmol}, 2.0$ equiv.), and the mixture was stirred at $25^{\circ} \mathrm{C}$ for $3 \mathrm{~h}$. Then, the solvent was removed under reduced pressure. Purification by column chromatography on silica gel eluting with hexanes/EtOAc (100\% hexanes to $1: 1$, containing $1 \%$ of $\mathrm{NEt}_{3}$ ) yielded 9-vinyl-9H-pyrido[3,4-b]indole (12) as a yellow oil (50.0 mg, $0.257 \mathrm{mmol}, 86 \%$ ).

$\mathbf{R}_{\boldsymbol{f}}=0.22$ (hexanes/EtOAc, 1:1).

\section{NMR Spectroscopy:}

${ }^{1} \mathrm{H}$ NMR $\left(600 \mathrm{MHz}, \mathrm{DMSO}-\mathrm{d}_{6}, 298 \mathrm{~K}, \delta\right): 9.27(\mathrm{~d}, J=0.8 \mathrm{~Hz}, 1 \mathrm{H}), 8.50(\mathrm{~d}, J=5.1 \mathrm{~Hz}, 1 \mathrm{H}), 8.35-8.30$ (m, $1 \mathrm{H}), 8.21(\mathrm{dd}, J=5.1,1.0 \mathrm{~Hz}, 1 \mathrm{H}), 7.95-7.92(\mathrm{~m}, 1 \mathrm{H}), 7.71-7.65(\mathrm{~m}, 2 \mathrm{H}), 7.39$ (ddd, $J=7.9,7.2,0.9 \mathrm{~Hz}$, $1 \mathrm{H}), 5.73(\mathrm{dd}, J=15.9,1.5 \mathrm{~Hz}, 1 \mathrm{H}), 5.22(\mathrm{dd}, J=9.3,1.5 \mathrm{~Hz}, 1 \mathrm{H})$.

${ }^{13} \mathrm{C}$ NMR (151 MHz, DMSO-d6, $\left.298 \mathrm{~K}, \delta\right): 140.5,139.4,134.9,133.9,129.4,129.2,129.0,122.0,121.4$, $121.4,114.7,111.6,102.5$.

HRMS-El (m/z) calculated for $\mathrm{C}_{13} \mathrm{H}_{10} \mathrm{~N}_{2}{ }^{+}[\mathrm{M}]^{+}, 194.0838$; found, 194.0839; deviation: $-0.5 \mathrm{ppm}$. 
5-Nitro-1-vinyl-1 H-indole (13)
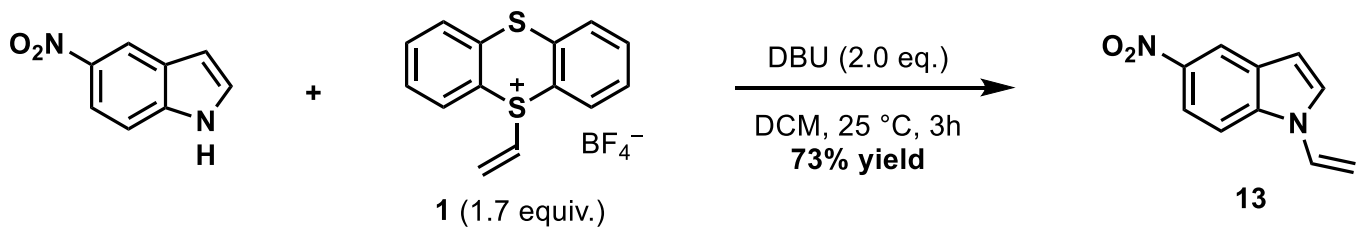

The title compound was prepared following general procedure A. Under air, a vial was charged with 5nitroindole (48.6 mg, $0.300 \mathrm{mmol}, 1.00$ equiv.) and 1 (168 mg, $0.510 \mathrm{mmol}, 1.70$ equiv.). DCM (3.0 mL, $c=0.10 \mathrm{M}$ ) was added, followed by DBU $(90 \mu \mathrm{L}, 92 \mathrm{mg}, 0.60 \mathrm{mmol}, 2.0$ equiv.), and the mixture was stirred at $25^{\circ} \mathrm{C}$ for $3 \mathrm{~h}$. Then, the solvent was removed under reduced pressure. Purification by column chromatography on silica gel eluting with hexanes/EtOAc (100\% hexanes to $10: 1$, containing $1 \%$ of $\mathrm{NEt}_{3}$ ) yielded 5-nitro-1-vinyl-1 $\mathrm{H}$-indole (13) as a yellow solid (41.3 mg, $0.219 \mathrm{mmol}, 73 \%)$.

$\mathbf{R}_{\boldsymbol{f}}=0.37$ (hexanes/EtOAc, $4: 1$ ).

\section{NMR Spectroscopy:}

${ }^{1} \mathrm{H}$ NMR $\left(500 \mathrm{MHz}, \mathrm{CDCl}_{3}, 298 \mathrm{~K}, \delta\right): 8.57(\mathrm{~d}, J=2.1 \mathrm{~Hz}, 1 \mathrm{H}), 8.16(\mathrm{dd}, J=9.1,2.2 \mathrm{~Hz}, 1 \mathrm{H}), 7.58(\mathrm{~d}$, $J=3.5 \mathrm{~Hz}, 1 \mathrm{H}$ ), $7.48(\mathrm{~d}, J=9.1 \mathrm{~Hz}, 1 \mathrm{H}), 7.23(\mathrm{dd}, J=15.7,8.9 \mathrm{~Hz}, 1 \mathrm{H}), 6.80(\mathrm{~d}, J=3.5 \mathrm{~Hz}, 1 \mathrm{H}), 5.34$ (dd, $J=15.7,1.8 \mathrm{~Hz}, 1 \mathrm{H}), 4.98$ (dd, $J=8.9,1.8 \mathrm{~Hz}, 1 \mathrm{H}$ ).

${ }^{13} \mathrm{C}$ NMR $\left(75 \mathrm{MHz}, \mathrm{CDCl}_{3}, 298 \mathrm{~K}, \delta\right): 142.6,138.1,129.1,128.5,126.7,118.3,118.2,109.6,106.8$, 100.2 .

HRMS-ESI (m/z) calculated for $\mathrm{C}_{10} \mathrm{H}_{8} \mathrm{~N}_{2} \mathrm{O}_{2} \mathrm{Na}^{+}[\mathrm{M}+\mathrm{Na}]^{+}, 211.0478$; found, 211.0478; deviation: 0.1 ppm.

1-Vinyl-1H-indole-3-carbaldehyde (14)

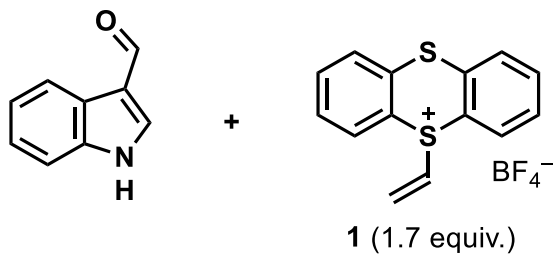

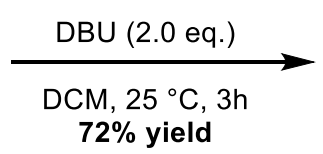

$72 \%$ yield

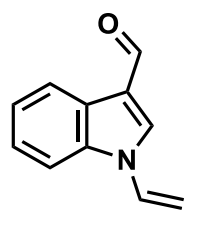

14

The title compound was prepared following general procedure A. Under air, a vial was charged with $1 \mathrm{H}$ indole-3-carbaldehyde (43.5 mg, $0.300 \mathrm{mmol}, 1.00$ equiv.) and 1 (168 mg, $0.510 \mathrm{mmol}, 1.70$ equiv.). DCM (3.0 mL, $c=0.10 \mathrm{M}$ ) was added, followed by DBU ( $90 \mu \mathrm{L}, 92 \mathrm{mg}, 0.60 \mathrm{mmol}, 2.0$ equiv.), and the mixture was stirred at $25^{\circ} \mathrm{C}$ for $3 \mathrm{~h}$. Then, the solvent was removed under reduced pressure. Purification by column chromatography on silica gel eluting with DCM/EtOAc (100\% DCM to $4: 1$, containing $1 \%$ of NEt 3 ) yielded 1vinyl-1 $\mathrm{H}$-indole-3-carbaldehyde (14) as a pale-yellow oil (37.2 mg, $0.217 \mathrm{mmol}, 72 \%)$.

$\mathbf{R}_{\boldsymbol{f}}=0.20(\mathrm{DCM})$.

\section{NMR Spectroscopy:}


${ }^{1} \mathrm{H}$ NMR $\left(500 \mathrm{MHz}, \mathrm{CDCl}_{3}, 298 \mathrm{~K}, \delta\right): 10.09(\mathrm{~s}, 1 \mathrm{H}), 8.34-8.29(\mathrm{~m}, 1 \mathrm{H}), 8.00(\mathrm{~s}, 1 \mathrm{H}), 7.51-7.48(\mathrm{~m}, 1 \mathrm{H})$, $7.41-7.33(\mathrm{~m}, 2 \mathrm{H}), 7.22$ (dd, $J=15.7,8.8 \mathrm{~Hz}, 1 \mathrm{H}), 5.47$ (dd, $J=15.7,1.9 \mathrm{~Hz}, 1 \mathrm{H}), 5.09$ (dd, $J=8.8$, $1.8 \mathrm{~Hz}, 1 \mathrm{H})$.

${ }^{13} \mathrm{C}$ NMR $\left(75 \mathrm{MHz}, \mathrm{CDCl}_{3}, 298 \mathrm{~K}, \delta\right): 185.1,136.7,133.7,129.2,125.5,124.9,123.8,122.4,120.2$, 110.1, 102.3.

HRMS-EI (m/z) calculated for $\mathrm{C}_{11} \mathrm{H}_{9} \mathrm{NO}^{+}[\mathrm{M}]^{+}, 171.0679$; found, 171.0679; deviation: $-0.1 \mathrm{ppm}$.

Ethyl 3-amino-1-vinyl-1H-pyrazole-4-carboxylate (15-I) and ethyl 5-amino-1-vinyl-1H-pyrazole-4carboxylate (15-II)
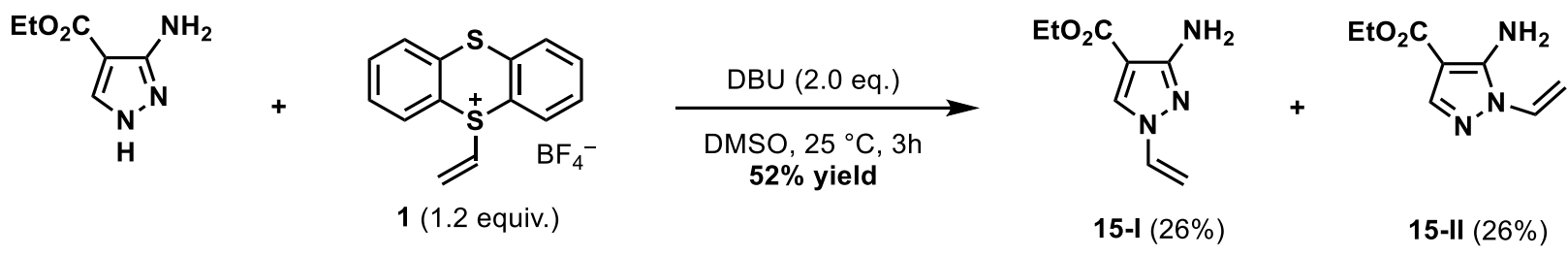

Under air, a vial was charged with ethyl 3-amino-1H-pyrazole-4-carboxylate (46.5 mg, $0.300 \mathrm{mmol}, 1.00$ equiv.) and DMSO (2.0 mL). DBU (90 $\mu \mathrm{L}, 92 \mathrm{mg}, 0.60 \mathrm{mmol}, 2.0$ equiv.) was added, and the mixture was stirred for $5 \mathrm{~min}$ at $25^{\circ} \mathrm{C}$. Then, a solution of 1 (119 mg, $0.360 \mathrm{mmol}, 1.20$ equiv.) in DMSO (1.0 mL) was added, and the resulting solution was stirred at at $25^{\circ} \mathrm{C}$ for 3 hours. The mixture was then diluted with DCM $(20 \mathrm{~mL})$ and washed with $\mathrm{H}_{2} \mathrm{O}(20 \mathrm{~mL})$. The aqueous phase was extracted with $\mathrm{DCM}(2 \times 10 \mathrm{~mL})$ and the combined organic phases were washed with brine $(20 \mathrm{~mL})$, dried over MgSO4 and the solvent removed under reduced pressure. Purification by column chromatography silica gel eluting with hexanes/EtOAc (8:1 to 3:1, containing $1 \%$ of $\mathrm{NEt}_{3}$ ) yielded ethyl 3-amino-1-vinyl-1 H-pyrazole-4-carboxylate (15-I) (14.0 mg, $0.077 \mathrm{mmol}, 26 \%$ ) and ethyl 5-amino-1-vinyl-1H-pyrazole-4-carboxylate (15-II) (14.0 mg, $0.077 \mathrm{mmol}, 26 \%$ ), both as a colorless solids.

\section{Data for 15-I:}

$\mathbf{R}_{\boldsymbol{f}}=0.36$ (hexanes/EtOAc, 3:1).

\section{NMR Spectroscopy:}

${ }^{1} \mathrm{H}$ NMR $\left(500 \mathrm{MHz}, \mathrm{CD}_{2} \mathrm{Cl}_{2}, 298 \mathrm{~K}, \delta\right): \delta 7.71(\mathrm{~s}, 1 \mathrm{H}), 6.78$ (dd, $\left.J=15.4,8.7 \mathrm{~Hz}, 1 \mathrm{H}\right), 5.48$ (dd, $J=15.4$, $1.0 \mathrm{~Hz}, 1 \mathrm{H}$ ), 4.81 (br s, 2H), 4.79 (dd, $J=8.7,1.0 \mathrm{~Hz}, 3 \mathrm{H}), 4.26$ (q, $J=7.1 \mathrm{~Hz}, 2 \mathrm{H}$ ), 1.32 (t, $J=7.2 \mathrm{~Hz}$, $3 \mathrm{H})$.

${ }^{13} \mathrm{C}$ NMR $\left(75 \mathrm{MHz}, \mathrm{CD}_{2} \mathrm{Cl}_{2}, 298 \mathrm{~K}, \delta\right): 164.2,157.1,132.3,131.9,101.5,100.4,60.4,14.6$.

HRMS-ESI (m/z) calculated for $\mathrm{C}_{8} \mathrm{H}_{12} \mathrm{~N}_{3} \mathrm{O}_{2}{ }_{2}[\mathrm{M}+\mathrm{H}]^{+}, 182.0924$; found, 182.0927; deviation: $-1.5 \mathrm{ppm}$.

\section{Data for 15-II:}

$\mathbf{R}_{\boldsymbol{f}}=0.29$ (hexanes/EtOAc, 3:1).

\section{NMR Spectroscopy:}


${ }^{1} \mathrm{H}$ NMR $\left(500 \mathrm{MHz}, \mathrm{CD}_{2} \mathrm{Cl}_{2}, 298 \mathrm{~K}, \delta\right.$ ): 7.66 (s, 1H), 6.80 (ddd, J=15.2, 8.9, $0.8 \mathrm{~Hz}, 1 \mathrm{H}$ ), 5.60 (dd, J = 15.4, 0.7 Hz, 1H), 5.26 (br s, 2H), 4.94 (dd, J=8.9, 0.7 Hz, 1H), 4.25 (q, J = 7.1 Hz, 2H), 1.32 (t, J = 7.1 $\mathrm{Hz}, 3 \mathrm{H})$.

${ }^{13} \mathrm{C}$ NMR $\left(151 \mathrm{MHz}, \mathrm{CD}_{2} \mathrm{Cl}_{2}, 298 \mathrm{~K}, \delta\right): 164.6,149.0,141.1,128.0,102.2,96.8,60.1,14.7$.

HRMS-El (m/z) calculated for $\mathrm{C}_{8} \mathrm{H}_{11} \mathrm{~N}_{3} \mathrm{O}_{2}+[\mathrm{M}]^{+}, 181.0846$; found, 181.0848; deviation: $-1.2 \mathrm{ppm}$.

\section{4-Bromo-3,5-dimethyl-1-vinyl-1H-pyrazole (16)}<smiles>Cc1[nH]cc([N])c1Br</smiles><smiles>C=C[S+]1c2ccccc2Sc2ccccc21</smiles>

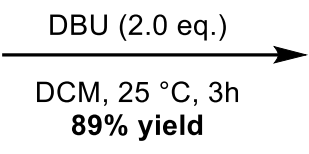

$\mathbf{8 9 \%}$ yield

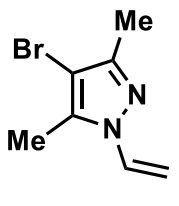

16

The title compound was prepared following general procedure A. Under air, a vial was charged with 4-bromo3,5-dimethyl-1 $\mathrm{H}$-pyrazole ( $52.5 \mathrm{mg}, 0.300 \mathrm{mmol}, 1.00$ equiv.) and 1 (168 mg, $0.510 \mathrm{mmol}, 1.70$ equiv.). DCM (3.0 mL, $c=0.10 \mathrm{M}$ ) was added, followed by DBU ( $90 \mu \mathrm{L}, 92 \mathrm{mg}, 0.60 \mathrm{mmol}, 2.0$ equiv.), and the mixture was stirred at $25^{\circ} \mathrm{C}$ for $3 \mathrm{~h}$. Then, the solvent was removed under reduced pressure. Purification by column chromatography on silica gel eluting with DCM (containing $1 \%$ of NEt $\mathrm{t}_{3}$ ) yielded 4-bromo-3,5-dimethyl-1-vinyl$1 \mathrm{H}$-pyrazole (16) as a colorless oil (53.5 mg, $0.266 \mathrm{mmol}, 89 \%)$.

$\mathbf{R} \boldsymbol{f}=0.30(\mathrm{DCM})$.

\section{NMR Spectroscopy:}

${ }^{1} \mathrm{H}$ NMR $\left(500 \mathrm{MHz}, \mathrm{CDCl}_{3}, 298 \mathrm{~K}, \delta\right): 6.87$ (dd, $\left.J=15.3,8.9 \mathrm{~Hz}, 1 \mathrm{H}\right), 5.62(\mathrm{~d}, J=15.3 \mathrm{~Hz}, 1 \mathrm{H}), 4.81$ (d, $J=8.9 \mathrm{~Hz}, 1 \mathrm{H}), 2.28(\mathrm{~s}, 3 \mathrm{H}), 2.25(\mathrm{~s}, 3 \mathrm{H})$.

${ }^{13} \mathrm{C}$ NMR $\left(75 \mathrm{MHz}, \mathrm{CDCl}_{3}, 298 \mathrm{~K}, \delta\right): 148.4,137.1,129.6,100.7,96.4,12.6,10.4$.

HRMS-El (m/z) calculated for $\mathrm{C}_{7} \mathrm{H}_{9} \mathrm{~N}_{2} \mathrm{Br}_{1}{ }^{+}[\mathrm{M}]^{+}, 199.9944$; found, 199.9945; deviation: -0.8 ppm.

Methyl 1-vinyl-1H-1,2,4-triazole-3-carboxylate (17)

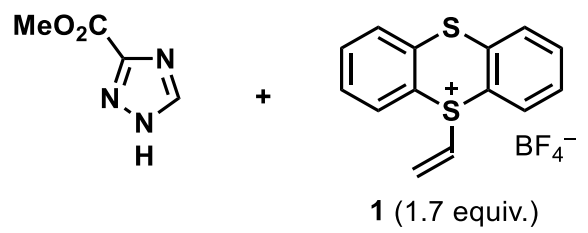

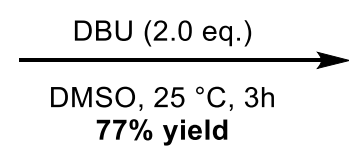

$77 \%$ yield

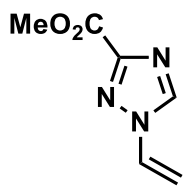

17

The title compound was prepared following general procedure A. Under air, a vial was charged with methyl 4H-1,2,4-triazole-3-carboxylate (38.1 mg, $0.300 \mathrm{mmol}, 1.00$ equiv.) and 1 (168 mg, $0.510 \mathrm{mmol}, 1.70$ equiv.). DMSO (3.0 mL, $c=0.10 \mathrm{M}$ ) was added, followed by DBU (90 $\mathrm{LL}, 92 \mathrm{mg}, 0.60 \mathrm{mmol}, 2.0$ equiv.), and the mixture was stirred at $25^{\circ} \mathrm{C}$ for $3 \mathrm{~h}$. Then, the solvent was removed under reduced pressure. Purification by column chromatography on silica gel eluting with DCM/EtOAc (100\% DCM to $2: 1$, containing $1 \%$ of $\mathrm{NEt}_{3}$ ) 
yielded methyl 1-vinyl-1 $\mathrm{H}-1,2,4$-triazole-3-carboxylate (17) as an off-white solid (35.3 mg, $0.231 \mathrm{mmol}, 77 \%$ ).

$\mathbf{R}_{\boldsymbol{f}}=0.27(\mathrm{DCM} / \mathrm{EtOAc}, 2: 1)$.

\section{NMR Spectroscopy:}

${ }^{1} \mathrm{H}$ NMR (500 MHz, $\left.\mathrm{CDCl}_{3}, 298 \mathrm{~K}, \delta\right): 8.36(\mathrm{~s}, 1 \mathrm{H}), 7.11$ (dd, $\left.J=15.6,8.8 \mathrm{~Hz}, 1 \mathrm{H}\right), 5.92$ (dd, $J=15.6,1.9$ $\mathrm{Hz}, 1 \mathrm{H}), 5.22(\mathrm{dd}, J=8.8,1.8 \mathrm{~Hz}, 1 \mathrm{H}), 3.99(\mathrm{~s}, 3 \mathrm{H})$.

${ }^{13} \mathrm{C}$ NMR $\left(126 \mathrm{MHz}, \mathrm{CDCl}_{3}, 298 \mathrm{~K}, \delta\right): 160.0,155.1,143.1,129.1,107.1,53.0$.

HRMS-ESI (m/z) calculated for $\mathrm{C}_{6} \mathrm{H}_{7} \mathrm{~N}_{3} \mathrm{O}_{2} \mathrm{Na}^{+}[\mathrm{M}+\mathrm{Na}]^{+}$, 176.0431; found, 176.0431; deviation: -0.2 ppm.

1-Vinylpyridin-4(1H)-one (18)<smiles>Oc1ccncc1</smiles>

1 (1.7 equiv.)

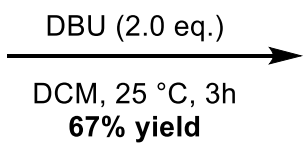

$67 \%$ yield

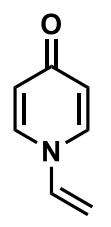

18

The title compound was prepared following general procedure A. Under air, a vial was charged with 4hydroxypyridine (28.5 mg, $0.300 \mathrm{mmol}, 1.00$ equiv.) and 1 (168 mg, $0.510 \mathrm{mmol}, 1.70$ equiv.). DCM (3.0 mL, $c=0.10 \mathrm{M}$ ) was added, followed by DBU $(90 \mu \mathrm{L}, 92 \mathrm{mg}, 0.60 \mathrm{mmol}, 2.0$ equiv.), and the mixture was stirred at $25{ }^{\circ} \mathrm{C}$ for $3 \mathrm{~h}$. Then, the solvent was removed under reduced pressure. Purification by column chromatography on silica gel eluting with $\mathrm{DCM} / \mathrm{MeOH}\left(50: 1\right.$ to $20: 1$, containing $1 \%$ of $\mathrm{NEt}_{3}$ ) yielded 1vinylpyridin-4(1H)-one (18) as a colorless solid (24.3 $\mathrm{mg}, 0.201 \mathrm{mmol}, 67 \%)$.

$\mathbf{R} \boldsymbol{f}=0.08(\mathrm{DCM} / \mathrm{MeOH}, 20: 1)$.

\section{NMR Spectroscopy:}

${ }^{1} \mathrm{H}$ NMR $\left(600 \mathrm{MHz}, \mathrm{CDCl}_{3}, 298 \mathrm{~K}, \delta\right): 7.52-7.49(\mathrm{~m}, 2 \mathrm{H}), 6.63(\mathrm{dd}, J=15.5,8.8 \mathrm{~Hz}, 1 \mathrm{H}), 6.43-6.39(\mathrm{~m}$, 2H), $5.20(\mathrm{dd}, J=15.6,2.9 \mathrm{~Hz}, 1 \mathrm{H}), 4.96(\mathrm{dd}, J=8.8,2.9 \mathrm{~Hz}, 1 \mathrm{H})$.

${ }^{13} \mathrm{C}$ NMR $\left(151 \mathrm{MHz}, \mathrm{CDCl}_{3}, 298 \mathrm{~K}, \delta\right): 179.7,136.3,135.6,119.0,101.7$.

HRMS-EI (m/z) calculated for $\mathrm{C}_{7} \mathrm{H}_{7} \mathrm{~N}_{1} \mathrm{O}_{1}{ }^{+}[\mathrm{M}]^{+}, 121.0522$; found, 121.0523; deviation: $-0.6 \mathrm{ppm}$.

\section{4-(4-Fluorophenyl)-1-vinyl-1H-imidazole (19)}
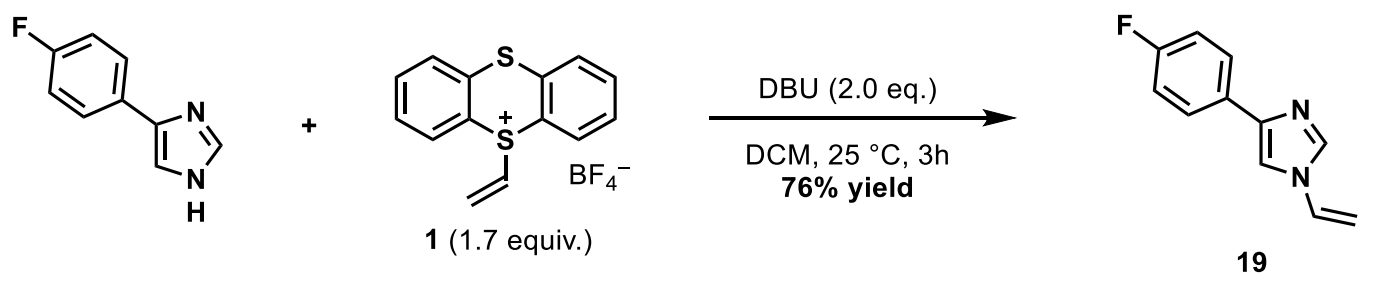

The title compound was prepared following general procedure A. Under air, a vial was charged with 4-(4- 
fluorophenyl)-1 $H$-imidazole (48.6 mg, $0.300 \mathrm{mmol}, 1.00$ equiv.) and 1 (168 mg, $0.510 \mathrm{mmol}, 1.70$ equiv.). $\operatorname{DCM}(3.0 \mathrm{~mL}, c=0.10 \mathrm{M}$ ) was added, followed by DBU ( $90 \mu \mathrm{L}, 92 \mathrm{mg}, 0.60 \mathrm{mmol}, 2.0$ equiv.), and the mixture was stirred at $25^{\circ} \mathrm{C}$ for $3 \mathrm{~h}$. Then, the solvent was removed under reduced pressure. Purification by column chromatography on silica gel eluting with hexanes/EtOAc $(100 \%$ hexanes to $1: 1$, containing $1 \%$ of $\mathrm{NEt}_{3}$ ) yielded 4-(4-fluorophenyl)-1-vinyl-1 $\mathrm{H}$-imidazole (19) as a colorless solid (43.0 mg, $0.228 \mathrm{mmol}, 76 \%$ ).

$\mathbf{R}_{\boldsymbol{f}}=0.27$ (hexanes/EtOAc, $1: 1$ ).

\section{NMR Spectroscopy:}

${ }^{1} \mathrm{H}$ NMR $\left(600 \mathrm{MHz}, \mathrm{CD}_{2} \mathrm{Cl}_{2}, 298 \mathrm{~K}, \delta\right): 7.79-7.75(\mathrm{~m}, 2 \mathrm{H}), 7.65(\mathrm{~d}, J=1.3 \mathrm{~Hz}, 1 \mathrm{H}), 7.47(\mathrm{~d}, J=1.3 \mathrm{~Hz}$, 1H), 7.10-7.06 (m, 2H), 6.94 (dd, $J=15.7,8.8 \mathrm{~Hz}, 1 \mathrm{H}), 5.34$ (dd, $J=15.7,1.8 \mathrm{~Hz}, 1 \mathrm{H}), 4.93$ (dd, $J=8.8$, $1.8 \mathrm{~Hz}, 1 \mathrm{H})$.

${ }^{13} \mathrm{C}$ NMR $\left(151 \mathrm{MHz}, \mathrm{CD}_{2} \mathrm{Cl}_{2}, 298 \mathrm{~K}, \delta\right): 162.5$ (d, $\left.J=245.0 \mathrm{~Hz}\right), 142.3,137.0,130.5(\mathrm{~d}, J=3.1 \mathrm{~Hz})$, 129.7, 127.0 (d, $J=7.9 \mathrm{~Hz}), 115.8(\mathrm{~d}, J=21.6 \mathrm{~Hz}), 111.1,101.6$.

${ }^{19} \mathrm{~F}$ NMR $\left(282 \mathrm{MHz}, \mathrm{CD}_{2} \mathrm{Cl}_{2}, 298 \mathrm{~K}, \delta\right):-116.2(\mathrm{~s})$.

HRMS-EI (m/z) calculated for $\mathrm{C}_{11} \mathrm{H}_{9} \mathrm{~N}_{2} \mathrm{~F}^{+}[\mathrm{M}]^{+}, 188.0744$; found, 188.0746; deviation: -0.9 ppm.

\section{4-Chloro-7-vinyl-7H-pyrrolo[2,3-d]pyrimidine (20)}

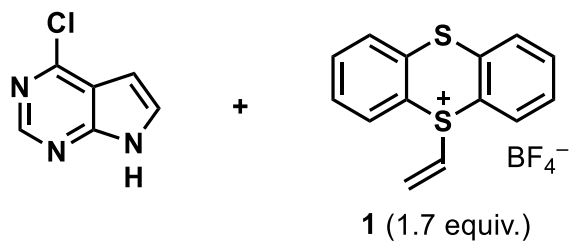

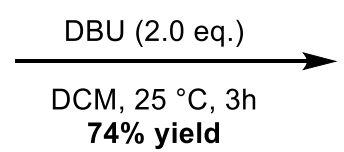

$74 \%$ yield

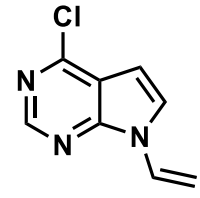

20

The title compound was prepared following general procedure A. Under air, a vial was charged with 4-chloro7H-pyrrolo[2,3-d]pyrimidine (46.1 mg, $0.300 \mathrm{mmol}, 1.00$ equiv.) and 1 (168 mg, $0.510 \mathrm{mmol}, 1.70$ equiv.). $\operatorname{DCM}(3.0 \mathrm{~mL}, C=0.10 \mathrm{M}$ ) was added, followed by DBU ( $90 \mu \mathrm{L}, 92 \mathrm{mg}, 0.60 \mathrm{mmol}, 2.0$ equiv.), and the mixture was stirred at $25^{\circ} \mathrm{C}$ for $3 \mathrm{~h}$. Then, the solvent was removed under reduced pressure. Purification by column chromatography on silica gel eluting with hexanes/EtOAc $(100 \%$ hexanes to $7: 1$, containing $1 \%$ of $\mathrm{NEt}_{3}$ ) yielded 4-chloro-7-vinyl-7 H-pyrrolo[2,3-d]pyrimidine (20) as a colorless solid (40.1 mg, $0.223 \mathrm{mmol}$, $74 \%)$.

$\mathbf{R}_{\boldsymbol{f}}=0.40$ (hexanes/EtOAc, $4: 1$ ).

\section{NMR Spectroscopy:}

${ }^{1} \mathrm{H}$ NMR (500 MHz, $\left.\mathrm{CDCl}_{3}, 298 \mathrm{~K}, \delta\right): 8.67(\mathrm{~s}, 1 \mathrm{H}), 7.59-7.49(\mathrm{~m}, 2 \mathrm{H}), 6.70(\mathrm{~d}, J=3.7 \mathrm{~Hz}, 1 \mathrm{H}), 5.48$ (dd, $J=16.0,1.7 \mathrm{~Hz}, 1 \mathrm{H}), 5.01$ (dd, $J=9.1,1.7 \mathrm{~Hz}, 1 \mathrm{H})$.

${ }^{13} \mathrm{C}$ NMR $\left(75 \mathrm{MHz}, \mathrm{CDCl}_{3}, 298 \mathrm{~K}, \delta\right): 152.6,151.6,150.6,127.8,125.0,118.4,101.9,100.5$.

HRMS-El (m/z) calculated for $\mathrm{C}_{8} \mathrm{H}_{6} \mathrm{~N}_{3} \mathrm{Cl}_{1}{ }^{+}[\mathrm{M}]^{+}, 179.0245$; found, 179.0247; deviation: -1.0 ppm. 
N-Vinyl-theophylline (21)<smiles>Cn1c(=O)c2[nH]cnc2n(C)c1=O</smiles>

theophylline<smiles>C=C[S+]1c2ccccc2Sc2ccccc21</smiles>

1 (1.7 equiv.)

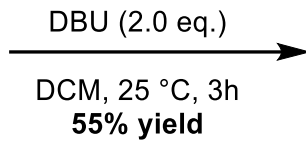

$\mathbf{5 5 \%}$ yield

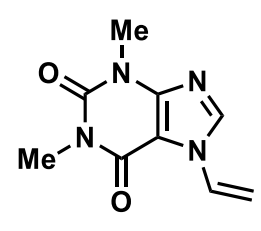

21

The title compound was prepared following general procedure A. Under air, a vial was charged with theophylline (54.0 mg, $0.300 \mathrm{mmol}, 1.00$ equiv.) and 1 (168 mg, $0.510 \mathrm{mmol}, 1.70$ equiv.). DCM (3.0 mL, $c=0.10 \mathrm{M}$ ) was added, followed by DBU ( $90 \mu \mathrm{L}, 92 \mathrm{mg}, 0.60 \mathrm{mmol}, 2.0$ equiv.), and the mixture was stirred at $25^{\circ} \mathrm{C}$ for $3 \mathrm{~h}$. Then, the solvent was removed under reduced pressure. Purification by column chromatography on silica gel eluting with DCM/EtOAc (100\% DCM to 1:1, containing $1 \%$ of $\mathrm{NEt}_{3}$ ) yielded $\mathrm{N}$ vinyl-theophylline (21) as a colorless solid $(34.0 \mathrm{mg}, 0.165 \mathrm{mmol}, 55 \%)$.

$\mathbf{R}_{\boldsymbol{f}}=0.19(\mathrm{DCM} / \mathrm{EtOAc}, 2: 1)$.

\section{NMR Spectroscopy:}

${ }^{1} \mathrm{H}$ NMR $\left(500 \mathrm{MHz}, \mathrm{CDCl}_{3}, 298 \mathrm{~K}, \delta\right): 7.92(\mathrm{~s}, 1 \mathrm{H}), 7.56$ (dd, J = 16.0, 8.9 Hz, 1H), 5.58 (dd, J = 16.0, 2.0 $\mathrm{Hz}, 1 \mathrm{H}), 5.14$ (dd, J=8.9, $2.0 \mathrm{~Hz}, 1 \mathrm{H}), 3.60(\mathrm{~s}, 3 \mathrm{H}), 3.41$ (s, 3H).

${ }^{13} \mathrm{C}$ NMR $\left(75 \mathrm{MHz}, \mathrm{CDCl}_{3}, 298 \mathrm{~K}, \delta\right): 155.2,151.6,149.2,137.5,128.7,106.4,105.2,30.0,28.2$.

HRMS-ESI (m/z) calculated for $\mathrm{C}_{9} \mathrm{H}_{10} \mathrm{~N}_{4} \mathrm{O}_{2} \mathrm{Na}_{1}{ }^{+}[\mathrm{M}+\mathrm{Na}]^{+}, 229.0696$; found, 229.0698; deviation: -0.8 ppm.

N-Vinyl,N'-Acetyl-L-tryptophan ethyl ester (22)

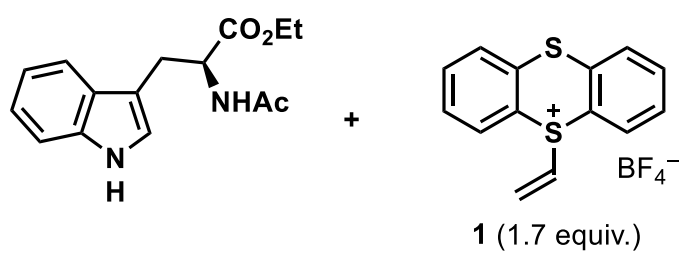

1 (1.7 equiv.)

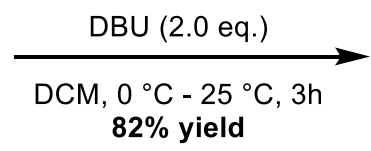

$82 \%$ yield

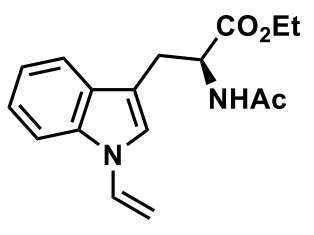

22

Under air, a vial was charged with $\mathrm{N}$-acetyl-L-tryptophan ethyl ester $(82.3 \mathrm{mg}, 0.300 \mathrm{mmol}, 1.00 \mathrm{equiv}$.) and $\operatorname{DCM}(3.0 \mathrm{~mL}, C=0.10 \mathrm{M})$. DBU $(90 \mu \mathrm{L}, 92 \mathrm{mg}, 0.60 \mathrm{mmol}, 2.0$ equiv.) was added, and the mixture was cooled to $0{ }^{\circ} \mathrm{C}$. At $0^{\circ} \mathrm{C}$, a solution of $1(168 \mathrm{mg}, 0.510 \mathrm{mmol}, 1.70$ equiv.) in DCM (1.0 mL) was added, and the resulting solution was stirred at $0{ }^{\circ} \mathrm{C}$ for $30 \mathrm{~min}$, followed by $2.5 \mathrm{~h}$ at $25^{\circ} \mathrm{C}$. After that, the solvent was removed under reduced pressure. Purification by column chromatography on silica gel eluting with DCM/EtOAc (5:1, containing $1 \%$ of $\left.\mathrm{NEt}_{3}\right)$ yielded $N$-vinyl, $N$ '-acetyl-L-tryptophan ethyl ester (22) as a colorless solid (74.0 mg, $0.246 \mathrm{mmol}, 82 \%)$.

$\mathbf{R}_{\boldsymbol{f}}=0.16(\mathrm{DCM} / \mathrm{EtOAc}, 5: 1)$.

\section{NMR Spectroscopy:}

${ }^{1} \mathrm{H}$ NMR $\left(500 \mathrm{MHz}, \mathrm{CDCl}_{3}, 298 \mathrm{~K}, \delta\right): 7.52(\mathrm{dt}, J=7.8,1.0 \mathrm{~Hz}, 1 \mathrm{H}), 7.43(\mathrm{~d}, J=8.2 \mathrm{~Hz}, 1 \mathrm{H}), 7.28-7.24$ 
$(\mathrm{m}, 1 \mathrm{H}), 7.22-7.13(\mathrm{~m}, 3 \mathrm{H}), 6.03(\mathrm{~d}, J=7.9 \mathrm{~Hz}, 1 \mathrm{H}), 5.10(\mathrm{dd}, J=15.7,1.4 \mathrm{~Hz}, 1 \mathrm{H}), 4.94(\mathrm{dt}, J=7.8$, $5.4 \mathrm{~Hz}, 1 \mathrm{H}), 4.73(\mathrm{dd}, J=8.9,1.4 \mathrm{~Hz}, 1 \mathrm{H}), 4.21-4.10(\mathrm{~m}, 2 \mathrm{H}), 3.37-3.26(\mathrm{~m}, 2 \mathrm{H}), 1.98(\mathrm{~s}, 3 \mathrm{H}), 1.22(\mathrm{t}$, $J=7.2 \mathrm{~Hz}, 3 \mathrm{H})$.

${ }^{13} \mathrm{C}$ NMR (151 MHz, $\left.\mathrm{CDCl}_{3}, 298 \mathrm{~K}, \delta\right): 172.0,169.8,135.8,129.4,129.2,123.2,121.9,120.8,119.2$, 113.0, 109.7, 96.3, 61.7, 53.0, 27.8, 23.4, 14.3.

HRMS-ESI (m/z) calculated for $\mathrm{C}_{17} \mathrm{H}_{19} \mathrm{~N}_{2} \mathrm{O}_{3}{ }^{-}[\mathrm{M}-\mathrm{H}]^{-}, 299.1401$; found, 299.1403; deviation: $-0.7 \mathrm{ppm}$.

N-Vinyl,N'-Boc-L-histidine methyl ester (23)

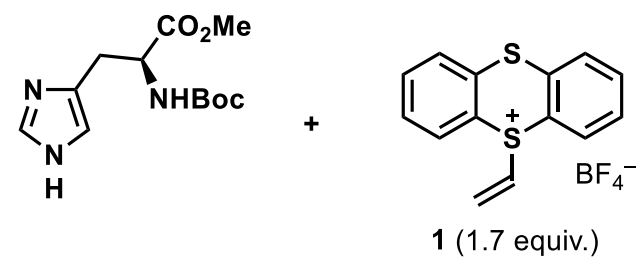

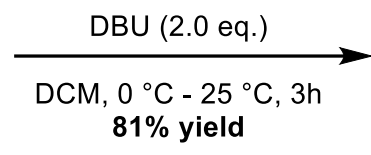

$81 \%$ yield

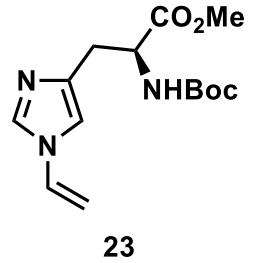

23

Under air, a vial was charged with $N$-Boc-L-histidine methyl ester ( $80.8 \mathrm{mg}, 0.300 \mathrm{mmol}, 1.00$ equiv.), 1 (168 mg, $0.510 \mathrm{mmol}, 1.70$ equiv.) and DCM (3.0 mL, $c=0.10 \mathrm{M}$ ), and the mixture was cooled to $0{ }^{\circ} \mathrm{C}$. DBU $\left(90 \mu \mathrm{L}, 92 \mathrm{mg}, 0.60 \mathrm{mmol}, 2.0\right.$ equiv.) was then added and the resulting solution was stirred at $0{ }^{\circ} \mathrm{C}$ for 30 min, followed by $2.5 \mathrm{~h}$ at $25^{\circ} \mathrm{C}$. After that, the solvent was removed under reduced pressure. Purification by column chromatography on silica gel eluting with $\mathrm{DCM} / \mathrm{MeOH}\left(100: 1\right.$ to $20: 1$, containing $1 \%$ of NEt $\mathrm{t}_{3}$ ) yielded $\mathrm{N}$-vinyl, $N$ '-Boc-L-histidine methyl ester (23) as an off-white solid (72.0 mg, $0.244 \mathrm{mmol}, 81 \%)$.

$\mathbf{R} \boldsymbol{f}=0.21(\mathrm{DCM} / \mathrm{MeOH}, 50: 1)$.

\section{NMR Spectroscopy:}

${ }^{\mathrm{s} 1} \mathrm{H}$ NMR $\left(600 \mathrm{MHz}, \mathrm{CDCl}_{3}, 298 \mathrm{~K}, \delta\right): 7.57$ (s, 1H), 6.95 (s, 1H), 6.81 (dd, $\left.J=15.7,8.8 \mathrm{~Hz}, 1 \mathrm{H}\right), 5.80$ (bs, $1 \mathrm{H}), 5.21$ (dd, $J=15.8,1.8 \mathrm{~Hz}, 1 \mathrm{H}), 4.85(\mathrm{dd}, J=8.8,1.8 \mathrm{~Hz}, 1 \mathrm{H}), 4.61-4.36(\mathrm{~m}, 1 \mathrm{H}), 3.69(\mathrm{~s}, 3 \mathrm{H})$, $3.14-2.96(\mathrm{~m}, 2 \mathrm{H}), 1.41(\mathrm{~s}, 9 \mathrm{H})$.

${ }^{13} \mathrm{C} \mathrm{NMR}\left(75 \mathrm{MHz}, \mathrm{CDCl}_{3}, 298 \mathrm{~K}, \delta\right): 172.4,155.5,138.5,135.8,129.1,113.3,101.5,79.6,53.3,52.2$, $30.2,28.2$.

HRMS-ESI (m/z) calculated for $\mathrm{C}_{14} \mathrm{H}_{22} \mathrm{~N}_{3} \mathrm{O}_{4}{ }^{+}[\mathrm{M}+\mathrm{H}]^{+}$, 296.1605; found, 296.1603; deviation: 0.5 ppm.

N-Vinyl-metaxalone (24)

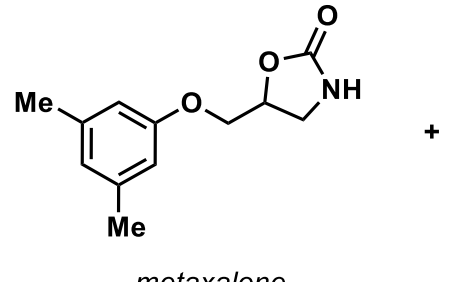

metaxalone

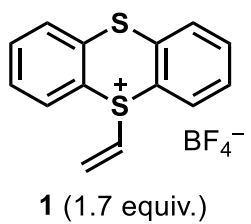

1 (1.7 equiv.)

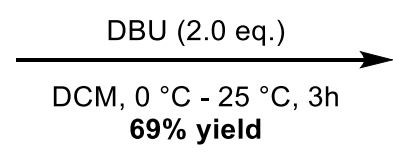

$69 \%$ yield

Under air, a vial was charged with metaxalone (66.4 mg, $0.300 \mathrm{mmol}, 1.00$ equiv.), 1 (168 mg, $0.510 \mathrm{mmol}$, 
1.70 equiv.) and $\mathrm{DCM}(3.0 \mathrm{~mL}, C=0.10 \mathrm{M})$, and the mixture was cooled to $0{ }^{\circ} \mathrm{C}$. DBU $(90 \mu \mathrm{L}, 92 \mathrm{mg}$, $0.60 \mathrm{mmol}, 2.0$ equiv.) was then added, and the resulting solution was stirred at $0{ }^{\circ} \mathrm{C}$ for $30 \mathrm{~min}$, followed by $2.5 \mathrm{~h}$ at $25^{\circ} \mathrm{C}$. After that, the solvent was removed under reduced pressure. Purification by column chromatography on silica gel eluting with hexanes/EtOAc (9:1 to $4: 1$, containing $1 \%$ of NEt3) yielded $N$-vinylmetaxalone (24) as a colorless solid (51 $\mathrm{mg}, 0.206 \mathrm{mmol}, 69 \%)$.

Reaction run at $1.00 \mathrm{mmol}$ scale: $181 \mathrm{mg}, 0.732 \mathrm{mmol}, 73 \%$.

$\mathbf{R}_{\boldsymbol{f}}=0.25$ (hexanes/EtOAc, 4:1).

\section{NMR Spectroscopy:}

${ }^{1} \mathrm{H}$ NMR $\left(500 \mathrm{MHz}, \mathrm{CDCl}_{3}, 298 \mathrm{~K}, \delta\right): 6.90$ (dd, J = 15.8, 8.9 Hz, 1H), $6.64(\mathrm{~s}, 1 \mathrm{H}), 6.53(\mathrm{~s}, 2 \mathrm{H}), 4.93$ (ddt, $J=9.1,5.8,4.7 \mathrm{~Hz}, 1 \mathrm{H}$ ), 4.45 (dd, $J=9.0,1.3 \mathrm{~Hz}, 1 \mathrm{H}$ ), 4.32 (dd, $J=15.9,1.3 \mathrm{~Hz}, 1 \mathrm{H}$ ), $4.16-4.10$ (m, 2H), $3.82(\mathrm{t}, J=9.2 \mathrm{~Hz}, 1 \mathrm{H}), 3.68(\mathrm{dd}, J=9.3,5.8 \mathrm{~Hz}, 1 \mathrm{H}), 2.29(\mathrm{~s}, 6 \mathrm{H})$.

${ }^{13} \mathrm{C}$ NMR $\left(75 \mathrm{MHz}, \mathrm{CDCl}_{3}, 298 \mathrm{~K}, \delta\right): 158.0,154.5,139.3,129.6,123.4,112.2,93.5,71.9,67.8,44.1$, 21.3.

HRMS-ESI (m/z) calculated for $\mathrm{C}_{14} \mathrm{H}_{18} \mathrm{NO}_{3}{ }^{+}[\mathrm{M}]^{+}, 248.1281$; found, 248.1281; deviation: -0.1 ppm.

$N$-Vinyl, $N$ '-Boc-carvedilol (25)

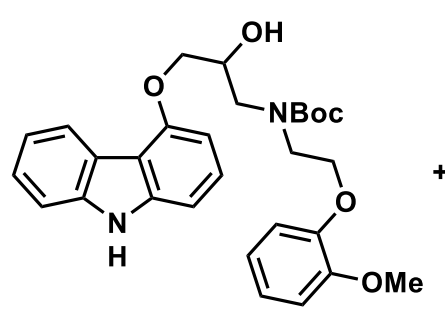

N-Boc-carvedilol

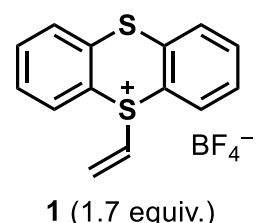

1 (1.7 equiv.)

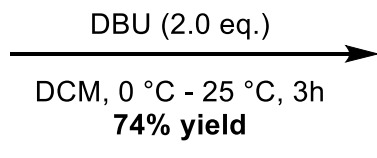

$74 \%$ yield

Under air, a vial was charged with N-Boc-carvedilol (151 mg, 0.300 mmol, 1.00 equiv.), 1 (168 mg, $0.510 \mathrm{mmol}, 1.70$ equiv.) and $\operatorname{DCM}(3.0 \mathrm{~mL}, c=0.10 \mathrm{M})$, and the mixture was cooled to $0^{\circ} \mathrm{C}$. $\mathrm{DBU}(90 \mu \mathrm{L}$, $92 \mathrm{mg}, 0.60 \mathrm{mmol}, 2.0$ equiv.) was then added, and the resulting solution was stirred at $0{ }^{\circ} \mathrm{C}$ for $30 \mathrm{~min}$, followed by $2.5 \mathrm{~h}$ at $25^{\circ} \mathrm{C}$. After that, the solvent was removed under reduced pressure. Purification by column chromatography on silica gel eluting with DCM/EtOAc (100:0 to 20:1, containing 1\% of NEt 3 ) yielded $N$-vinyl-N'-Boc-carvedilol (25) as a colorless solid (118 mg, $0.226 \mathrm{mmol}, 74 \%$ ).

$\mathbf{R}_{\boldsymbol{f}}=0.41$ (DCM/EtOAc, 9:1).

\section{NMR Spectroscopy:}

${ }^{1} \mathrm{H}$ NMR (300 MHz, CD2 $\left.\mathrm{Cl}_{2}, 298 \mathrm{~K}, \delta\right): 8.48-8.32(\mathrm{~m}, 1 \mathrm{H}), 7.66(\mathrm{~d}, J=8.2 \mathrm{~Hz}, 1 \mathrm{H}), 7.49-7.22(\mathrm{~m}, 5 \mathrm{H})$, $6.97-6.83(\mathrm{~m}, 4 \mathrm{H}), 6.80(\mathrm{~d}, J=7.9 \mathrm{~Hz}, 1 \mathrm{H}), 5.57$ (dd, J=16.0, $0.9 \mathrm{~Hz}, 1 \mathrm{H}), 5.19$ (dd, J = 9.2, $0.9 \mathrm{~Hz}$, $1 \mathrm{H}), 4.55-4.43(\mathrm{~m}, 1 \mathrm{H}), 4.39-4.13(\mathrm{~m}, 4 \mathrm{H}), 3.89-3.67(\mathrm{~m}, 3 \mathrm{H}), 3.75(\mathrm{~s}, 3 \mathrm{H}), 1.49(\mathrm{bs}, 9 \mathrm{H})$.

${ }^{13} \mathrm{C}$ NMR (75 MHz, $\mathrm{CD}_{2} \mathrm{Cl}_{2}, 298$ K, $\left.\delta\right): 157.7,155.6,149.7,148.5,141.2,139.0,130.0,127.4,125.7$ 
$123.6,121.9,121.2,121.1,121.0,113.9,113.3,112.2,110.3,103.9,103.0,102.6,80.9,70.8,70.5$,

$68.1,55.9,53.7,49.5,28.5$.

HRMS-ESI (m/z) calculated for $\mathrm{C}_{31} \mathrm{H}_{16} \mathrm{~N}_{2} \mathrm{O}_{6} \mathrm{Na}^{+}[\mathrm{M}+\mathrm{Na}]^{+}$, 555.2466; found, 555.2468; deviation: -0.4 ppm.

$\mathrm{N}$-Vinyl-coumarin 7 (26)<smiles>CCNc1ccc2cc(-c3nc4ccccc4[nH]3)c(=O)oc2c1</smiles>

coumarin 7<smiles>C=C[S+]1c2ccccc2Sc2ccccc21</smiles>

1 (1.7 equiv.)

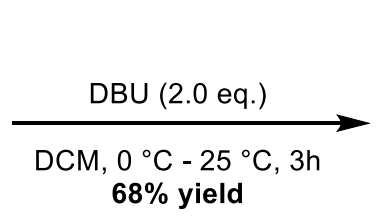

$68 \%$ yield

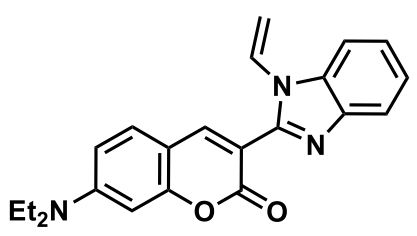

26

Under air, a vial was charged with coumarin 7 (100 mg, $0.300 \mathrm{mmol}, 1.00$ equiv.), 1 (168 mg, $0.510 \mathrm{mmol}$, 1.70 equiv.) and $\mathrm{DCM}(3.0 \mathrm{~mL}, C=0.10 \mathrm{M})$, and the mixture was cooled to $0{ }^{\circ} \mathrm{C}$. DBU $(90 \mu \mathrm{L}, 92 \mathrm{mg}$, $0.60 \mathrm{mmol}, 2.0$ equiv.) was then added, and the resulting solution was stirred at $0{ }^{\circ} \mathrm{C}$ for $30 \mathrm{~min}$, followed by $2.5 \mathrm{~h}$ at $25^{\circ} \mathrm{C}$. After that, the solvent was removed under reduced pressure. Purification by column chromatography on silica gel eluting with DCM/EtOAc (100:0 to $20: 1$, containing $1 \%$ of NEt 3 ) yielded $\mathrm{N}$-vinylcoumarin 7 (26) as a bright yellow solid (73 $\mathrm{mg}, 0.21 \mathrm{mmol}, 68 \%)$.

$\mathbf{R}_{\boldsymbol{f}}=0.42(\mathrm{DCM} / \mathrm{EtOAc}, 9: 1)$.

\section{NMR Spectroscopy:}

${ }^{1} \mathrm{H}$ NMR $\left(500 \mathrm{MHz}, \mathrm{CDCl}_{3}, 298 \mathrm{~K}, \delta\right): 7.83-7.77(\mathrm{~m}, 1 \mathrm{H}), 7.68-7.62(\mathrm{~m}, 1 \mathrm{H}), 7.36(\mathrm{~d}, J=8.9 \mathrm{~Hz}, 1 \mathrm{H})$, $7.35-7.29(\mathrm{~m}, 2 \mathrm{H}), 7.13(\mathrm{dd}, J=15.8,8.9 \mathrm{~Hz}, 1 \mathrm{H}), 6.62(\mathrm{dd}, J=8.9,2.5 \mathrm{~Hz}, 1 \mathrm{H}), 6.53(\mathrm{~d}, J=2.5 \mathrm{~Hz}$, $1 \mathrm{H}), 5.54(\mathrm{dd}, J=15.7,1.2 \mathrm{~Hz}, 1 \mathrm{H}), 5.20(\mathrm{dd}, J=8.9,1.2 \mathrm{~Hz}, 1 \mathrm{H}), 3.45(\mathrm{q}, J=7.2 \mathrm{~Hz}, 4 \mathrm{H}), 1.24(\mathrm{t}, J=$ $7.1 \mathrm{~Hz}, 6 \mathrm{H})$.

${ }^{13} \mathrm{C}$ NMR $\left(75 \mathrm{MHz}, \mathrm{CDCl}_{3}, 298 \mathrm{~K}, \delta\right):$ 159.9, 157.6, 151.9, 149.0, 147.3, 143.2, 134.5, 130.3, 130.1, $123.7,123.0,119.9,111.6,110.4,109.5,108.2,107.0,97.0,45.0,12.4$.

HRMS-ESI (m/z) calculated for $\mathrm{C}_{22} \mathrm{H}_{22} \mathrm{~N}_{3} \mathrm{O}_{2}{ }^{+}[\mathrm{M}+\mathrm{H}]^{+}, 360.1707$; found, 360.1710; deviation: -1.0 ppm.

N-Vinyl-lansoprazole (27)

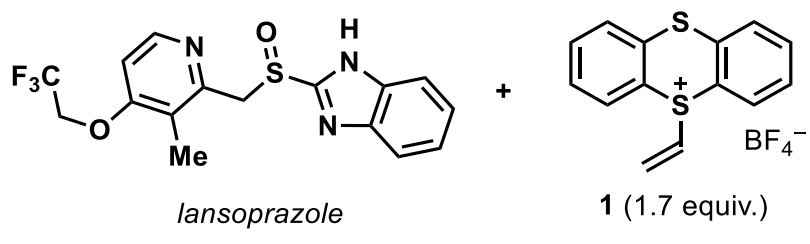

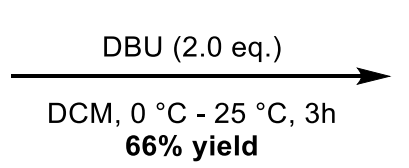

$66 \%$ yield

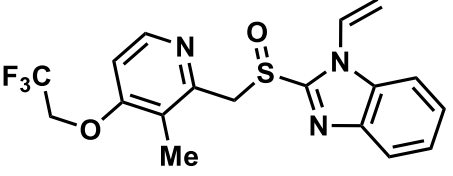

27

Under air, a vial was charged with lansoprazole (111 mg, $0.300 \mathrm{mmol}, 1.00$ equiv.) and DCM (3.0 mL). DBU $\left(90 \mu \mathrm{L}, 92 \mathrm{mg}, 0.60 \mathrm{mmol}, 2.0\right.$ equiv.) was added, and the mixture was cooled to $0^{\circ} \mathrm{C}$. At $0{ }^{\circ} \mathrm{C}$, a solution of 1 (168 mg, $0.510 \mathrm{mmol}, 1.70$ equiv.) in DCM $(1.0 \mathrm{~mL})$ was added, and the resulting solution was stirred at $0{ }^{\circ} \mathrm{C}$ for $30 \mathrm{~min}$, followed by $2.5 \mathrm{~h}$ at $25^{\circ} \mathrm{C}$. After that, the solvent was removed under reduced pressure. 
Purification by column chromatography on silica gel eluting with DCM/EtOAc (1:2, containing $1 \%$ of NEt 3 ) yielded $N$-vinyl-lansoprazole (27) as a colorless solid (78.1 $\mathrm{mg}, 0.198 \mathrm{mmol}, 66 \%)$.

$\mathbf{R} \boldsymbol{f}=0.15(\mathrm{DCM} / \mathrm{EtOAC}, 1: 2)$.

\section{NMR Spectroscopy:}

${ }^{1} \mathrm{H}$ NMR $\left(300 \mathrm{MHz}, \mathrm{CD}_{2} \mathrm{Cl}_{2}, 298 \mathrm{~K}, \delta\right): 8.18(\mathrm{~d}, J=5.7 \mathrm{~Hz}, 1 \mathrm{H}), 7.82-7.77(\mathrm{~m}, 1 \mathrm{H}), 7.71-7.64(\mathrm{~m}, 1 \mathrm{H})$, 7.50 (dd, $J=15.8,8.9 \mathrm{~Hz}, 1 \mathrm{H}), 7.46-7.33(\mathrm{~m}, 2 \mathrm{H}), 6.63(\mathrm{~d}, J=5.7 \mathrm{~Hz}, 1 \mathrm{H}), 5.70$ (dd, $J=15.8,1.4 \mathrm{~Hz}$, $1 \mathrm{H}), 5.37(\mathrm{dd}, J=8.9,1.4 \mathrm{~Hz}, 1 \mathrm{H}), 5.06-4.84(\mathrm{~m}, 2 \mathrm{H}), 4.41(\mathrm{q}, J=8.0 \mathrm{~Hz}, 2 \mathrm{H}), 2.25(\mathrm{~s}, 3 \mathrm{H})$.

${ }^{13} \mathrm{C}$ NMR $\left(75 \mathrm{MHz}, \mathrm{CD}_{2} \mathrm{Cl}_{2}, 298 \mathrm{~K}, \delta\right): 162.1,153.2,152.0,148.5,142.9,134.9,128.6,125.7,124.3$, $123.5(q, J=277.7 \mathrm{~Hz}), 123.1,121.5,112.4,109.2,106.3,65.8$ (q, $J=36.2 \mathrm{~Hz}), 59.7,11.0$.

${ }^{19} \mathrm{~F}$ NMR $\left(282 \mathrm{MHz}, \mathrm{CD}_{2} \mathrm{Cl}_{2}, 298 \mathrm{~K}, \delta\right):-74.3$ (s).

HRMS-ESI (m/z) calculated for $\mathrm{C}_{18} \mathrm{H}_{17} \mathrm{~N}_{3} \mathrm{O}_{2} \mathrm{~S}_{1} \mathrm{~F}_{3}{ }^{+}[\mathrm{M}+\mathrm{H}]^{+}$, 396.0988; found, 396.0990; deviation: -0.5 ppm.

\section{Reaction optimization of the vinylation of organoboron compounds}

\section{General procedure for optimization of reaction conditions}

Under ambient atmosphere, a $4 \mathrm{~mL}$ vial equipped with a teflon-coated magnetic stirring bar was charged with 4-biphenylboronic acid (S4, 9.9 mg, 0.050 mmol, 1.0 equiv.), Pd(dba)2 (1.4 mg, 2.5 umol, 5.0 mol\%), P(o-tol) 3 (1.7 mg, $5.5 \mu \mathrm{mol}, 11 \mathrm{~mol} \%$ ) and $t$-BuOLi $(6.0 \mathrm{mg}, 0.075 \mathrm{mmol}, 1.5$ equiv.). The vial was transferred into a $\mathrm{N}_{2}$-filled glove box. Subsequently, dry THF $(0.5 \mathrm{~mL}, \mathrm{C}=0.1 \mathrm{M})$ was added into the vial. The reaction mixture was stirred for $2 \mathrm{~min}$ at $25^{\circ} \mathrm{C}$ before 1 (24.8 mg, $0.0750 \mathrm{mmol}, 1.50$ equiv.) was added into the vial in one portion. The vial was capped and was then transferred out of the glove box. The vial was placed on a heating block preheated at $60^{\circ} \mathrm{C}$ where the reaction mixture was stirred for $16 \mathrm{~h}$. The reaction mixture was cooled to $25^{\circ} \mathrm{C}$ and filtered through a pad of celite eluting with DCM $(4 \mathrm{~mL})$. The filtrate was collected and concentrated under reduced pressure. To the residue was added dibromomethane $(7.0 \mu \mathrm{L}, 17 \mathrm{mg}, 0.10 \mathrm{mmol})$ as an internal standard. The ${ }^{1} \mathrm{H}$ NMR resonances of the vinyl protons of the product between 5.1 and 6.0 ppm were integrated relative to the ${ }^{1} \mathrm{H}$ NMR resonances of the protons of dibromomethane $(\delta=4.90 \mathrm{ppm})$.

Table S1: Optimization of yield as a function of catalyst

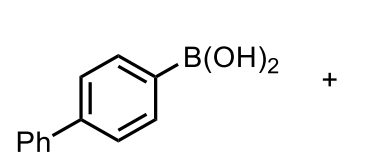

S4

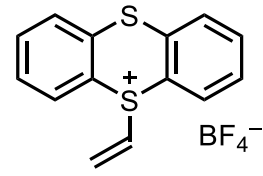

1

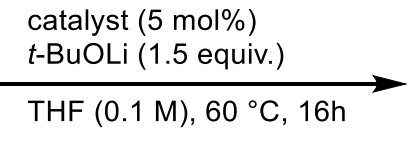

Yield

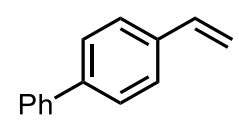

S5

\begin{tabular}{llc}
\hline Entry & Catalyst & Yield \\
\hline 1 & No catalyst & $0 \%$
\end{tabular}




\begin{tabular}{|c|c|c|}
\hline 2 & $\mathrm{Pd}(\mathrm{OAc})_{2}+11 \mathrm{~mol} \% \mathrm{PPh}_{3}$ & $47 \%$ \\
\hline 3 & $\mathrm{Pd}(\mathrm{OAc})_{2}+11 \mathrm{~mol} \% \mathrm{P}(\mathrm{o} \text {-tol })_{3}$ & $50 \%$ \\
\hline 4 & $\mathrm{Pd}(\mathrm{OAc})_{2}+11 \mathrm{~mol} \% \mathrm{P}(2 \text {-fur })_{3}$ & $39 \%$ \\
\hline 5 & $\mathrm{Pd}(\mathrm{dba})_{2}+11 \mathrm{~mol} \% \mathrm{PPh}_{3}$ & $45 \%$ \\
\hline 6 & $\mathrm{Pd}(\mathrm{dba})_{2}+11 \mathrm{~mol} \% \mathrm{P}(\mathrm{o}-\mathrm{tol})_{3}$ & $60 \%$ \\
\hline 7 & $\mathrm{Pd}(\mathrm{dba})_{2}+11 \mathrm{~mol} \% \mathrm{P}(2 \text {-fur })_{3}$ & $43 \%$ \\
\hline 8 & $\mathrm{Pd}(\mathrm{dppf}) \mathrm{Cl}_{2}$ & $5 \%$ \\
\hline 9 & $\mathrm{Pd}\left(\mathrm{PCy}_{3}\right)_{2} \mathrm{Cl}_{2}$ & $0 \%$ \\
\hline 10 & $\mathrm{Pd}(\mathrm{PtBu})_{2}$ & $42 \%$ \\
\hline 11 & $\mathrm{Pd}\left(\mathrm{PPh}_{3}\right)_{4}$ & $40 \%$ \\
\hline
\end{tabular}

Table S2: Optimization of yield as a function of base

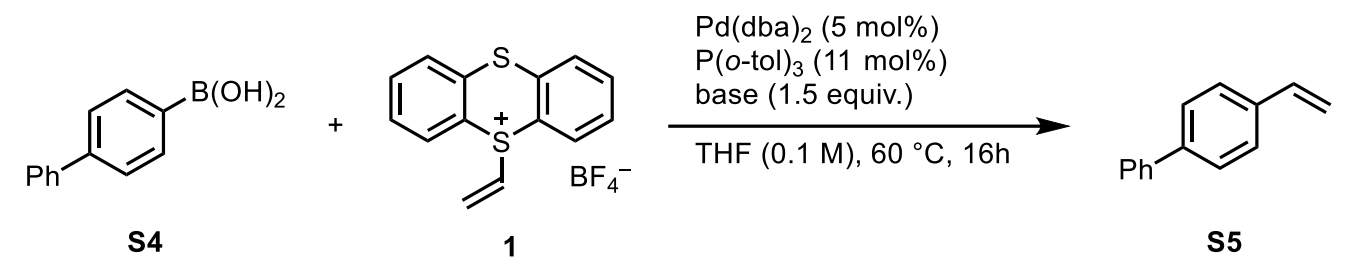

\begin{tabular}{lll}
\hline Entry & Base & Yield \\
\hline 1 & No base & $<5 \%$ \\
2 & $\mathrm{Na}_{2} \mathrm{CO}_{3}$ & $11 \%$ \\
3 & $\mathrm{Cs}_{2} \mathrm{CO}_{3}$ & $76 \%$ \\
4 & $\mathrm{~K}_{3} \mathrm{PO}_{4}$ & $60 \%$ \\
5 & $\mathrm{CsF}$ & $75 \%$ \\
6 & $t$-BuOLi & $81 \%$ \\
7 & $t$-BuONa & $<5 \%$ \\
8 & $t$-BuOK & $10 \%$ \\
9 & TMSOK & $11 \%$ \\
\hline
\end{tabular}


Table S3: Optimization of yield as a function of concentration

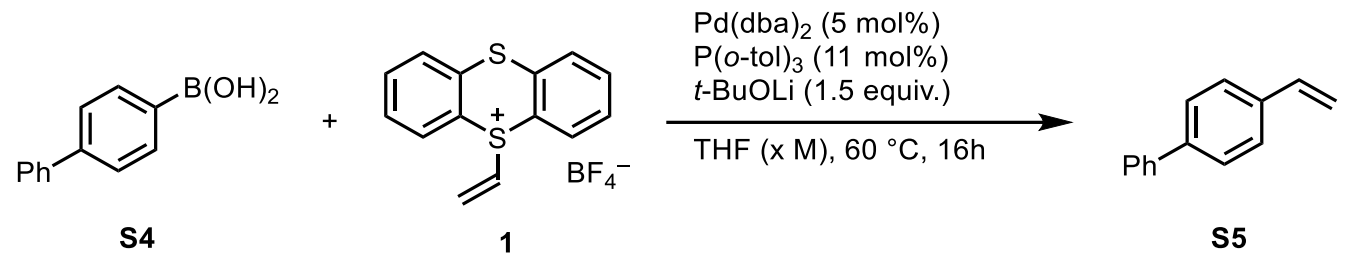

\begin{tabular}{lll}
\hline Entry & Concentration & Yield \\
\hline 1 & $0.025 \mathrm{M}$ & $52 \%$ \\
2 & $0.05 \mathrm{M}$ & $90 \%$ \\
3 & $0.1 \mathrm{M}$ & $81 \%$ \\
4 & $0.2 \mathrm{M}$ & $68 \%$ \\
\hline
\end{tabular}

\section{Suzuki-type vinylation of aryl organoboron compounds using vinyl-TT+ 1}

\section{General procedure B}

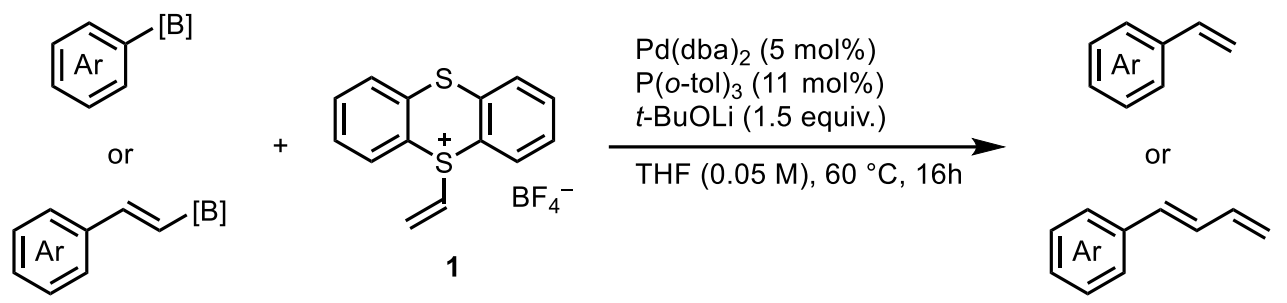

Under ambient atmosphere, a $20 \mathrm{~mL}$ vial equipped with a teflon-coated magnetic stirring bar was charged with organoboron species ( $0.300 \mathrm{mmol}, 1.00$ equiv.), $\mathrm{Pd}(\mathrm{dba})_{2}(8.6 \mathrm{mg}, 15 \mu \mathrm{mol}, 5.0 \mathrm{~mol} \%), \mathrm{P}(o$-tol)3 $(10.0 \mathrm{mg}, 33.0 \mu \mathrm{mol}, 11.0 \mathrm{~mol} \%$ ) and $t$-BuOLi (36.0 mg, $0.450 \mathrm{mmol}, 1.50$ equiv.). The vial was transferred into a $\mathrm{N}_{2}$-filled glove box. Subsequently, dry THF $(6 \mathrm{~mL}, \mathrm{C}=0.05 \mathrm{M})$ was added into the vial. The reaction mixture was stirred for $2 \mathrm{~min}$ at $25^{\circ} \mathrm{C}$ before 1 (149 mg, $0.450 \mathrm{mmol}, 1.50$ equiv.) was added into the vial in one portion. The vial was capped and was then transferred out of the glove box. The vial was placed on a heating block preheated at $60{ }^{\circ} \mathrm{C}$ where the reaction mixture was stirred for $16 \mathrm{~h}$. The reaction mixture was cooled to $25^{\circ} \mathrm{C}$ and filtered through a pad of celite eluting with DCM $(20 \mathrm{~mL})$. The filtrate was collected and concentrated under vacuum to roughly $5 \mathrm{~mL}$. Silica gel (approximately $300 \mathrm{mg}$ ) was added, and the mixture was evaporated to dryness under reduced pressure. The residue was purified by column chromatography on silica gel to give corresponding product. [Note: unless otherwise mentioned, $t$-BuOLi stored at ambient atmosphere was used. When extra-dry t-BuOLi stored in the glovebox was used, poor yields were obtained]. 
General procedure for the vinylation of organoboron compounds using a Schlenk line

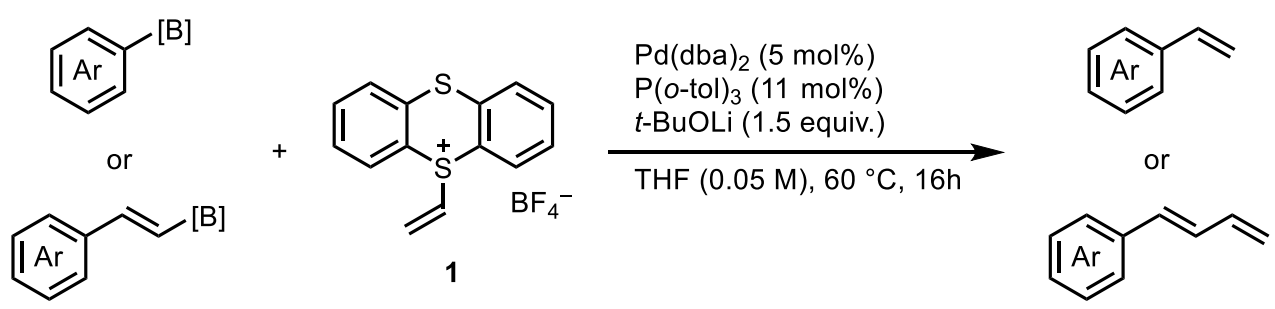

Under ambient atmosphere, a $20 \mathrm{~mL}$ Schlenk tube equipped with a teflon-coated magnetic stirring bar was charged with organoboron species ( $0.300 \mathrm{mmol}, 1.00$ equiv.), $\mathrm{Pd}(\mathrm{dba})_{2}(8.6 \mathrm{mg}, 15 \mu \mathrm{mol}, 5.0 \mathrm{~mol} \%), \mathrm{P}(o \text {-tol })_{3}$ $(10.0 \mathrm{mg}, 33.0 \mu \mathrm{mol}, 11.0 \mathrm{~mol} \%)$ and $t$-BuOLi (36.0 mg, $0.450 \mathrm{mmol}, 1.50$ equiv.). The Schlenk tube was evacuated and backfilled with argon. Subsequently, dry THF ( $6 \mathrm{~mL}, \mathrm{c}=0.05 \mathrm{M})$ was added into the Schlenk tube via a syringe. The reaction mixture was stirred for $2 \mathrm{~min}$ at $25^{\circ} \mathrm{C}$ before 1 ( $149 \mathrm{mg}, 0.450 \mathrm{mmol}$, 1.50 equiv.) was added into the Schlenk tube in one portion. The Schlenk tube was placed in an oil bath preheated at $60^{\circ} \mathrm{C}$ where the reaction mixture was stirred for $16 \mathrm{~h}$. The reaction mixture was cooled to $25^{\circ} \mathrm{C}$ and filtered through a pad of celite eluting with DCM $(20 \mathrm{~mL})$. The filtrate was collected and concentrated under vacuum to roughly $5 \mathrm{~mL}$. Silica gel (approximately $300 \mathrm{mg}$ ) was added, and the mixture was evaporated to dryness under reduced pressure. The residue was purified by column chromatography on silica gel to give corresponding product.

1-Methyl-3-vinylbenzene (28)

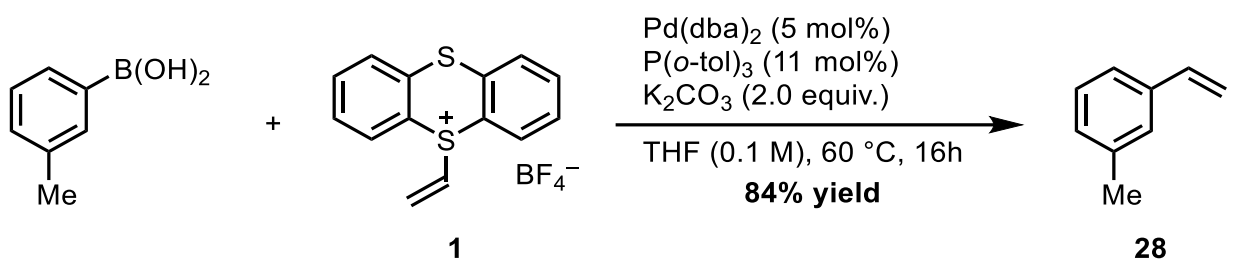

Under ambient atmosphere, a $4 \mathrm{~mL}$ vial equipped with a teflon-coated magnetic stirring bar was charged with 3-methylbenzeneboronic acid (6.8 mg, $0.050 \mathrm{mmol}, 1.0$ equiv.), $\mathrm{Pd}(\mathrm{dba})_{2}(1.4 \mathrm{mg}, 2.5 \mu \mathrm{mol}, 5.0 \mathrm{~mol} \%), \mathrm{P}(\mathrm{o}-$ tol) $3(1.7 \mathrm{mg}, 5.5 \mu \mathrm{mol}, 11 \mathrm{~mol} \%)$ and $t$-BuOLi $(6.0 \mathrm{mg}, 0.075 \mathrm{mmol}, 1.5$ equiv.). The vial was transferred into a $\mathrm{N}_{2}$-filled glove box. Subsequently, dry THF $(0.5 \mathrm{~mL}, \mathrm{C}=0.1 \mathrm{M})$ was added into the vial. The reaction mixture was stirred for $2 \mathrm{~min}$ at $25^{\circ} \mathrm{C}$ before $1(24.8 \mathrm{mg}, 0.0750 \mathrm{mmol}, 1.50$ equiv.) was added into the vial in one portion. The vial was capped and was then transferred out of the glove box. The vial was placed on a heating block preheated at $60{ }^{\circ} \mathrm{C}$ where the reaction mixture was stirred for $16 \mathrm{~h}$. The reaction mixture was cooled to $25^{\circ} \mathrm{C}$ and filtered through a pad of celite eluting with DCM $(4 \mathrm{~mL})$. The filtrate was collected and concentrated under reduced pressure. Due to the volatility of the title product, its yield was determined via NMR analysis of the reaction mixture. To the residue was added dibromomethane $(7.0 \mu \mathrm{L}, 17 \mathrm{mg}, 0.10 \mathrm{mmol}$, 2.0 equiv.) as an internal standard. The ${ }^{1} \mathrm{H}$ NMR resonances of the vinyl protons of the product between 5.6 and $5.8 \mathrm{ppm}$ were integrated relative to the ${ }^{1} \mathrm{H}$ NMR resonances of the protons of dibromomethane $(\delta=$ $4.90 \mathrm{ppm}$ ). The yield was determined as $84 \%$ (Figure S9). 


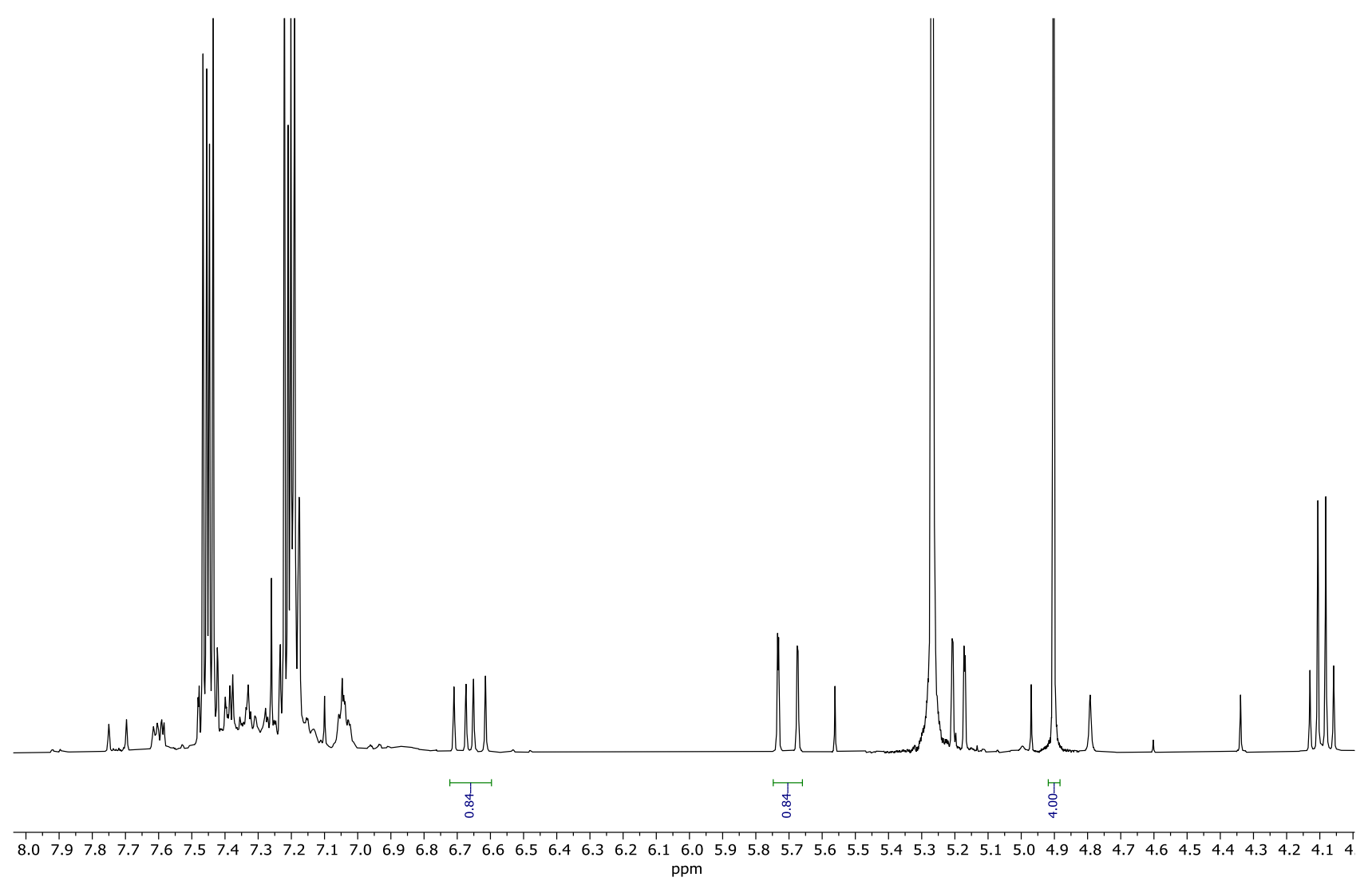

Figure S9. Determination of the yield of 28 via ${ }^{1} \mathrm{H}$ NMR analysis, $\mathrm{CDCl}_{3}, 300 \mathrm{MHz}, 298 \mathrm{~K}$.

\section{3-Methyl-4-vinylbenzonitrile (29)}<smiles>C=C[S+]1c2ccccc2Sc2ccccc21</smiles>

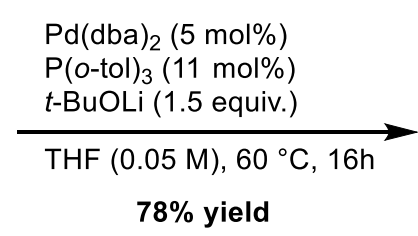

$78 \%$ yield<smiles>C=Cc1ccc(C#N)cc1[N+](=O)[O-]</smiles>

29

The title compound was prepared following general procedure B. Under ambient atmosphere, a $20 \mathrm{~mL}$ vial equipped with a teflon-coated magnetic stirring bar was charged with 2-methyl-4-cyanophenylboronic acid

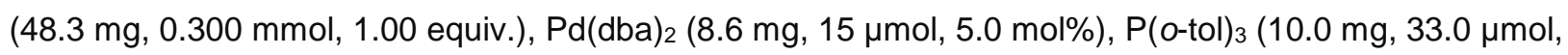
$11.0 \mathrm{~mol} \%$ ) and $t$-BuOLi (36.0 mg, $0.450 \mathrm{mmol}, 1.50$ equiv.). The vial was transferred into a $\mathrm{N}_{2}$-filled glove box. Subsequently, dry THF $(6 \mathrm{~mL}, \mathrm{c}=0.05 \mathrm{M})$ was added into the vial. The reaction mixture was stirred for $2 \mathrm{~min}$ at $25^{\circ} \mathrm{C}$ before 1 (149 mg, $0.450 \mathrm{mmol}, 1.50$ equiv.) was added into the vial in one portion. The vial was capped and was then transferred out of the glove box. The vial was placed on a heating block preheated at $60{ }^{\circ} \mathrm{C}$ where the reaction mixture was stirred for $16 \mathrm{~h}$. The reaction mixture was cooled to $25^{\circ} \mathrm{C}$ and filtered through a pad of celite eluting with DCM $(20 \mathrm{~mL})$. The filtrate was collected and concentrated under vacuum to roughly $5 \mathrm{~mL}$. Silica gel (approximately $300 \mathrm{mg}$ ) was added, and the mixture was evaporated to dryness under reduced pressure. The residue was purified by column chromatography on silica gel eluting with a 
solvent mixture of EtOAc:pentane (1:50 (v:v)) to afford $33.5 \mathrm{mg}$ of the title compound (29) as a colorless oil (78\% yield).

$\mathbf{R}_{\boldsymbol{f}}=0.35$ (EtOAc:pentane, 1:19 (v:v)).

\section{NMR Spectroscopy:}

${ }^{1} \mathrm{H}$ NMR $\left(500 \mathrm{MHz}, \mathrm{CDCl}_{3}, 298 \mathrm{~K}, \delta\right): 7.54(\mathrm{~m}, 1 \mathrm{H}), 7.47-7.38(\mathrm{~m}, 2 \mathrm{H}), 6.91(\mathrm{dd}, J=17.4,11.1 \mathrm{~Hz}, 1 \mathrm{H})$, $5.75(\mathrm{dd}, J=17.4,1.0 \mathrm{~Hz}, 1 \mathrm{H}$ ), 5.47 (dd, $J=11.0,1.0 \mathrm{~Hz}, 1 \mathrm{H}), 2.37$ (s, 3H) ppm.

${ }^{13} \mathrm{C}$ NMR (126 MHz, $\left.\mathrm{CDCl}_{3}, 298 \mathrm{~K}, \delta\right): 141.6,136.6,133.8,133.6,129.9,126.1,119.2,118.7,111.1$, $19.6 \mathrm{ppm}$.

HRMS-EI (m/z) calculated for $\mathrm{C}_{10} \mathrm{H}_{9} \mathrm{~N}_{1}{ }^{+}[\mathrm{M}]^{+}, 143.0731$; found, 143.0729; deviation: $-1.1 \mathrm{ppm}$.

1-Chloro-4-methoxy-2-vinylbenzene (30)

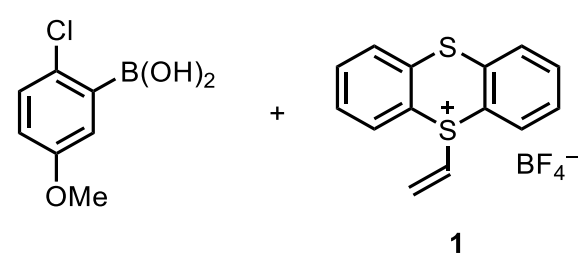

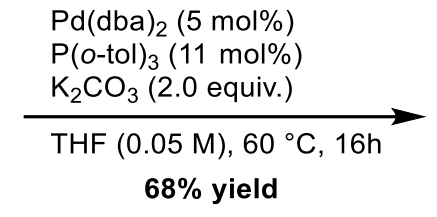

$68 \%$ yield

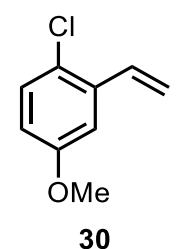

30

The title compound was prepared following general procedure B. Under ambient atmosphere, a $20 \mathrm{~mL}$ vial equipped with a teflon-coated magnetic stirring bar was charged with 2-chloro-5-methoxyphenylboronic acid (55.9 mg, 0.300 mmol, 1.00 equiv.), Pd(dba)2 (8.6 mg, $15 \mu \mathrm{mol}, 5.0 \mathrm{~mol} \%$ ), P(o-tol)3 $(10.0 \mathrm{mg}, 33.0 \mu \mathrm{mol}$, $11.0 \mathrm{~mol} \%$ ) and $\mathrm{K}_{2} \mathrm{CO}_{3}\left(82.9 \mathrm{mg}, 0.600 \mathrm{mmol}, 2.00\right.$ equiv.). The vial was transferred into a $\mathrm{N}_{2}$-filled glove box. Subsequently, dry THF $(6 \mathrm{~mL}, \mathrm{C}=0.05 \mathrm{M})$ was added into the vial. The reaction mixture was stirred for 2 min at $25^{\circ} \mathrm{C}$ before 1 (149 mg, $0.450 \mathrm{mmol}, 1.50$ equiv.) was added into the vial in one portion. The vial was capped and was then transferred out of the glove box. The vial was placed on a heating block preheated at $60{ }^{\circ} \mathrm{C}$ where the reaction mixture was stirred for $16 \mathrm{~h}$. The reaction mixture was cooled to $25^{\circ} \mathrm{C}$ and filtered through a pad of celite eluting with DCM $(20 \mathrm{~mL})$. The filtrate was collected and concentrated under vacuum to roughly $5 \mathrm{~mL}$. Silica gel (approximately $300 \mathrm{mg}$ ) was added, and the mixture was evaporated to dryness under reduced pressure. The residue was purified by column chromatography on silica gel eluting with pentane to afford $34.3 \mathrm{mg}$ of the title compound (30) as a colorless oil (68\% yield).

$\mathbf{R}_{\boldsymbol{f}}=0.40$ (EtOAc:pentane, 1:19 (v:v)).

\section{NMR Spectroscopy:}

'H NMR $\left(500 \mathrm{MHz}, \mathrm{CDCl}_{3}, 298 \mathrm{~K}, \delta\right): 7.25(\mathrm{~d}, J=8.8 \mathrm{~Hz}, 1 \mathrm{H}), 7.15-6.98(\mathrm{~m}, 2 \mathrm{H}), 6.77$ (dd, $J=8.8$, $3.0 \mathrm{~Hz}, 1 \mathrm{H}), 5.73$ (dd, $J=17.5,1.1 \mathrm{~Hz}, 1 \mathrm{H}), 5.38$ (dd, $J=11.0,1.1 \mathrm{~Hz}, 1 \mathrm{H}), 3.81$ (s, 3H) ppm.

${ }^{13} \mathrm{C}$ NMR $\left(126 \mathrm{MHz}, \mathrm{CDCl}_{3}, 298 \mathrm{~K}, \delta\right): 158.5,136.6,133.5,130.4,125.0,116.7,115.0,111.7,55.7$ ppm. HRMS-El (m/z) calculated for $\mathrm{C}_{9} \mathrm{H}_{9} \mathrm{O}_{1} \mathrm{Cl}_{1}{ }^{+}[\mathrm{M}]^{+}, 168.0337$; found, 168.0336; deviation: $-0.6 \mathrm{ppm}$. 
1-Chloro-4-vinylbenzene (31)

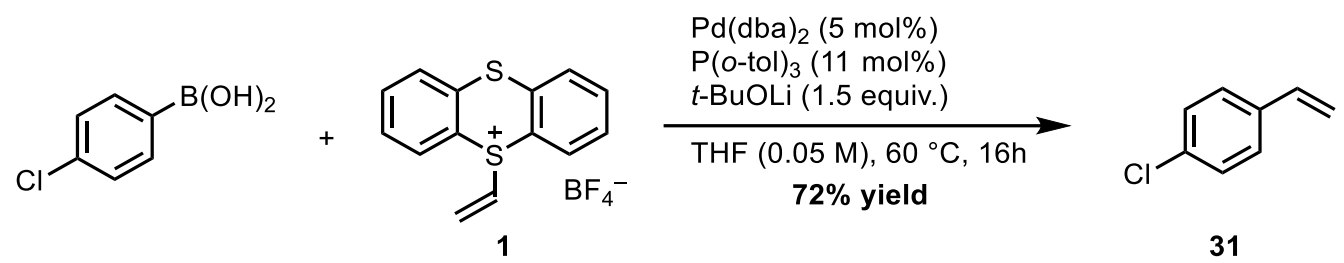

The title compound was prepared following general procedure B. Under ambient atmosphere, a $20 \mathrm{~mL}$ vial equipped with a teflon-coated magnetic stirring bar was charged with 4-chlorophenylboronic acid (46.9 mg, 0.300 mmol, 1.00 equiv.), Pd(dba) 2 (8.6 mg, $15 \mu \mathrm{mol}, 5.0$ mol\%), $\mathrm{P}(o-\mathrm{tol})_{3}(10.0 \mathrm{mg}, 33.0 \mu \mathrm{mol}, 11.0 \mathrm{~mol} \%)$ and $t$-BuOLi (36.0 mg, $0.450 \mathrm{mmol}, 1.50$ equiv.). The vial was transferred into a $\mathrm{N}_{2}$-filled glove box. Subsequently, dry THF $(6 \mathrm{~mL}, \mathrm{c}=0.05 \mathrm{M})$ was added into the vial. The reaction mixture was stirred for 2 min at $25^{\circ} \mathrm{C}$ before 1 (149 mg, $0.450 \mathrm{mmol}, 1.50$ equiv.) was added into the vial in one portion. The vial was capped and was then transferred out of the glove box. The vial was placed on a heating block preheated at $60{ }^{\circ} \mathrm{C}$ where the reaction mixture was stirred for $16 \mathrm{~h}$. The reaction mixture was cooled to $25^{\circ} \mathrm{C}$ and filtered through a pad of celite eluting with DCM $(20 \mathrm{~mL})$. The filtrate was collected and concentrated under vacuum to roughly $5 \mathrm{~mL}$. Silica gel (approximately $300 \mathrm{mg}$ ) was added, and the mixture was evaporated to dryness under reduced pressure. The residue was purified by column chromatography on silica gel eluting with pentane to afford $34.3 \mathrm{mg}$ of the title compound (31) as a colorless oil (72\% yield).

$\mathbf{R} \boldsymbol{f}=0.51$ (pentane)

\section{NMR Spectroscopy:}

'H NMR (300 MHz, $\left.\mathrm{CDCl}_{3}, 298 \mathrm{~K}, \delta\right): 7.43-7.27(\mathrm{~m}, 4 \mathrm{H}), 6.67$ (dd, $\left.J=17.6,10.9 \mathrm{~Hz}, 1 \mathrm{H}\right), 5.73(\mathrm{dd}, J=$ 17.6, $0.8 \mathrm{~Hz}, 1 \mathrm{H}), 5.27$ (dd, $J=10.9,0.8 \mathrm{~Hz}, 1 \mathrm{H}$ ) ppm.

${ }^{13} \mathrm{C}$ NMR $\left(75 \mathrm{MHz}, \mathrm{CDCl}_{3}, 298 \mathrm{~K}, \delta\right):$ 136.2, 135.8, 133.6, 128.8, 127.6, $114.6 \mathrm{ppm}$.

HRMS-EI (m/z) calculated for $\mathrm{C}_{8} \mathrm{H}_{7} \mathrm{Cl}_{1}{ }^{+}[\mathrm{M}]^{+}, 138.0232$; found, 138.0230; deviation: $-0.8 \mathrm{ppm}$.

\section{1-Chloro-4-(vinyl- $\left.d_{3}\right)$ benzene $\left(31-d_{3}\right)$}
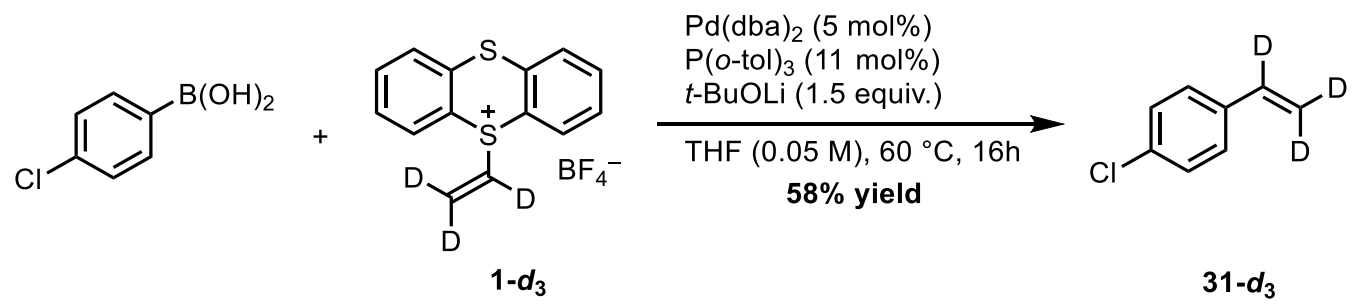

Under ambient atmosphere, a $4 \mathrm{~mL}$ vial equipped with a teflon-coated magnetic stirring bar was charged with 4-chlorophenylboronic acid (7.8 mg, 0.050 mmol, 1.0 equiv.), Pd(dba)2 (1.4 mg, 2.5 umol, 5.0 mol\%), P(o-tol)3 (1.7 mg, $5.5 \mu \mathrm{mol}, 11 \mathrm{~mol} \%$ ) and $t$-BuOLi $(6.0 \mathrm{mg}, 0.075 \mathrm{mmol}, 1.5$ equiv.). The vial was transferred into a $\mathrm{N}_{2}$-filled glove box. Subsequently, dry THF ( $\left.1 \mathrm{~mL}, \mathrm{c}=0.05 \mathrm{M}\right)$ was added into the vial. The reaction mixture 
was stirred for $2 \mathrm{~min}$ at $25^{\circ} \mathrm{C}$ before $1-d_{3}(25.0 \mathrm{mg}, 0.0750 \mathrm{mmol}, 1.50$ equiv.) was added into the vial in one portion. The vial was capped and was then transferred out of the glove box. The vial was placed on a heating block preheated at $60^{\circ} \mathrm{C}$ where the reaction mixture was stirred for $16 \mathrm{~h}$. The reaction mixture was cooled to $25^{\circ} \mathrm{C}$ and filtered through a pad of celite eluting with DCM $(5 \mathrm{~mL})$. Silica gel (approximately $50 \mathrm{mg}$ ) was added to the filtrate, and the mixture was evaporated to dryness under reduced pressure. The residue was purified by column chromatography on silica gel eluting with pentane to afford $4.1 \mathrm{mg}$ of the title compound $\left(\mathbf{3 1}-\boldsymbol{d}_{3}\right)$ as a colorless oil $(58 \%$ yield).

$\mathbf{R} \boldsymbol{f}=0.52$ (pentane).

\section{NMR Spectroscopy:}

${ }^{1} \mathrm{H}$ NMR $\left(300 \mathrm{MHz}, \mathrm{CD}_{2} \mathrm{Cl}_{2}, 298 \mathrm{~K}, \delta\right): 7.42-7.34(\mathrm{~m}, 2 \mathrm{H}), 7.33-7.26(\mathrm{~m}, 2 \mathrm{H}) \mathrm{ppm}$.

${ }^{2} \mathrm{H}$ NMR (92 MHz, $\left.\mathrm{CD}_{2} \mathrm{Cl}_{2}, 298 \mathrm{~K}, \delta\right): 6.71(\mathrm{~s}, 1 \mathrm{H}), 5.76(\mathrm{~s}, 1 \mathrm{H}), 5.30$ (s, 1H) ppm.

${ }^{13} \mathrm{C}$ NMR $\left(151 \mathrm{MHz}, \mathrm{CD}_{2} \mathrm{Cl}_{2}, 298 \mathrm{~K}, \delta\right): 136.5,135.5$ (t, $\left.J=24.9 \mathrm{~Hz}\right), 133.7,129.0,127.9,114.2$

(m) ppm.

HRMS-El (m/z) calculated for $\mathrm{C}_{8} \mathrm{H}_{4} \mathrm{D}_{3} \mathrm{Cl}_{1}{ }^{+}[\mathrm{M}]^{+}, 141.0421$; found, 141.0419; deviation: $-1.6 \mathrm{ppm}$.

\section{1-Ethoxy-2-vinylbenzene (32)}

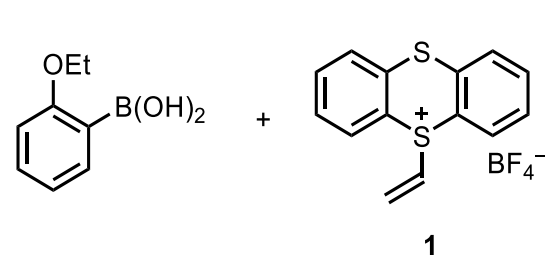

1

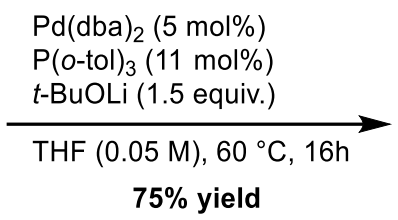

$75 \%$ yield

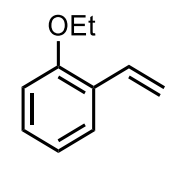

32

The title compound was prepared following general procedure B. Under ambient atmosphere, a $20 \mathrm{~mL}$ vial equipped with a teflon-coated magnetic stirring bar was charged with 2-ethoxyphenylboronic acid (49.8 mg, $0.300 \mathrm{mmol}, 1.00$ equiv.), Pd(dba)2 (8.6 mg, $15 \mu \mathrm{mol}, 5.0 \mathrm{~mol} \%), \mathrm{P}(\mathrm{o} \text {-tol })_{3}(10.0 \mathrm{mg}, 33.0 \mu \mathrm{mol}, 11.0 \mathrm{~mol} \%)$ and $t$-BuOLi (36.0 mg, $0.450 \mathrm{mmol}, 1.50$ equiv.). The vial was transferred into a $\mathrm{N}_{2}$-filled glove box.

Subsequently, dry THF ( $6 \mathrm{~mL}, \mathrm{c}=0.05 \mathrm{M})$ was added into the vial. The reaction mixture was stirred for $2 \mathrm{~min}$ at $25^{\circ} \mathrm{C}$ before 1 (149 mg, $0.450 \mathrm{mmol}, 1.50$ equiv.) was added into the vial in one portion. The vial was capped and was then transferred out of the glove box. The vial was placed on a heating block preheated at $60{ }^{\circ} \mathrm{C}$ where the reaction mixture was stirred for $16 \mathrm{~h}$. The reaction mixture was cooled to $25^{\circ} \mathrm{C}$ and filtered through a pad of celite eluting with DCM $(20 \mathrm{~mL})$. The filtrate was collected and concentrated under vacuum to roughly $5 \mathrm{~mL}$. Silica gel (approximately $300 \mathrm{mg}$ ) was added, and the mixture was evaporated to dryness under reduced pressure. The residue was purified by column chromatography on silica gel eluting with pentane to afford $33.3 \mathrm{mg}$ of the title compound (32) as a colorless oil (75\% yield).

$\mathbf{R} \boldsymbol{f}=0.51$ (EtOAc:pentane, 1:19 (v:v)).

\section{NMR Spectroscopy:}


${ }^{1} \mathrm{H}$ NMR $\left(300 \mathrm{MHz}, \mathrm{CDCl}_{3}, 298 \mathrm{~K}, \delta\right): 7.86(\mathrm{dd}, J=7.7,1.8 \mathrm{~Hz}, 1 \mathrm{H}), 7.60$ (ddd, $J=8.2,7.4,1.7 \mathrm{~Hz}, 1 \mathrm{H}$ ), 7.46 (dd, $J=17.9,11.2 \mathrm{~Hz}, 1 \mathrm{H}$ ), 7.31 (td, $J=7.5,0.6 \mathrm{~Hz}, 1 \mathrm{H}), 7.24$ (dd, $J=8.3,1.1 \mathrm{~Hz}, 1 \mathrm{H}$ ), 6.14 (dd, $J$ $=17.8,1.6 \mathrm{~Hz}, 1 \mathrm{H}), 5.64(\mathrm{dd}, J=11.2,1.6 \mathrm{~Hz}, 1 \mathrm{H}), 4.45(\mathrm{q}, J=7.0 \mathrm{~Hz}, 2 \mathrm{H}), 1.83(\mathrm{t}, J=7.0 \mathrm{~Hz}$, 3H) ppm.

${ }^{13} \mathrm{C} \mathrm{NMR}\left(75 \mathrm{MHz}, \mathrm{CDCl}_{3}, 298 \mathrm{~K}, \delta\right): 156.3,132.0,128.9,127.0,126.7,120.7,114.4,112.2,64.0$, $15.0 \mathrm{ppm}$.

HRMS-EI (m/z) calculated for $\mathrm{C}_{10} \mathrm{H}_{12} \mathrm{O}_{1}{ }^{+}[\mathrm{M}]^{+}, 148.0885$; found, 148.0883; deviation: -1.3 ppm.

\section{1-Bromo-4-vinylbenzene (33)}

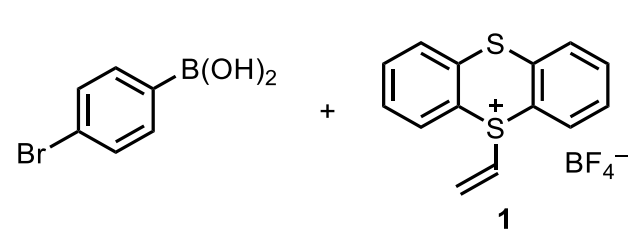

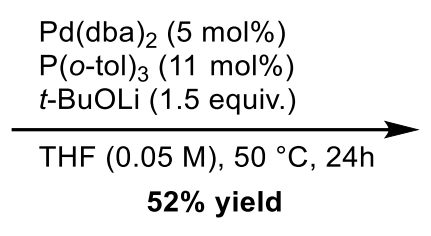

$52 \%$ yield

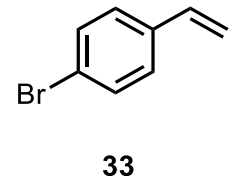

33

Under ambient atmosphere, a $20 \mathrm{~mL}$ vial equipped with a teflon-coated magnetic stirring bar was charged with 1 (168 mg, $0.510 \mathrm{mmol}, 1.70$ equiv.), $\mathrm{Pd}(\mathrm{dba})_{2}$ (8.6 mg, 15 mol, $5.0 \mathrm{~mol} \%$ ), $\mathrm{P}(\mathrm{o}$-tol) 3 (10.0 mg, $33.0 \mu \mathrm{mol}, 11.0 \mathrm{~mol} \%$ ) and $t$-BuOLi (36.0 mg, $0.450 \mathrm{mmol}, 1.50$ equiv.). The vial was transferred into a $\mathrm{N}_{2}-$ filled glove box. Subsequently, dry THF $(6 \mathrm{~mL}, \mathrm{c}=0.05 \mathrm{M})$ was added into the vial. The reaction mixture was stirred for $2 \mathrm{~min}$ at $25^{\circ} \mathrm{C}$ before 4-bromophenylboronic acid $(60.2 \mathrm{mg}, 0.300 \mathrm{mmol}, 1.00$ equiv.) was added into the vial in one portion. The vial was capped and was then transferred out of the glove box. The vial was placed on a heating block preheated at $50^{\circ} \mathrm{C}$ where the reaction mixture was stirred for $24 \mathrm{~h}$. The reaction mixture was cooled to $25^{\circ} \mathrm{C}$ and filtered through a pad of celite eluting with DCM (20 mL). The filtrate was collected and concentrated under vacuum to roughly $5 \mathrm{~mL}$. Silica gel (approximately $300 \mathrm{mg}$ ) was added, and the mixture was evaporated to dryness under reduced pressure. The residue was purified by column chromatography on silica gel eluting with pentane to afford $28.5 \mathrm{mg}$ of the title compound (33) as a colorless oil $(52 \%$ yield).

$\mathbf{R}_{\boldsymbol{f}}=0.51$ (pentane).

\section{NMR Spectroscopy:}

${ }^{1} \mathrm{H}$ NMR $\left(500 \mathrm{MHz}, \mathrm{CDCl}_{3}, 298 \mathrm{~K}, \delta\right): 7.49-7.40(\mathrm{~m}, 2 \mathrm{H}), 7.32-7.24(\mathrm{~m}, 2 \mathrm{H}), 6.66(\mathrm{dd}, J=17.6$, $10.8 \mathrm{~Hz}, 1 \mathrm{H}), 5.74(\mathrm{dd}, J=17.6,0.7 \mathrm{~Hz}, 1 \mathrm{H}), 5.28(\mathrm{dd}, J=11.0,0.7 \mathrm{~Hz}, 1 \mathrm{H}) \mathrm{ppm}$.

${ }^{13} \mathrm{C}$ NMR (126 MHz, $\left.\mathrm{CDCl}_{3}, 298 \mathrm{~K}, \delta\right): 136.6,135.9,131.8,127.9,121.7,114.8$ ppm.

HRMS-EI (m/z) calculated for $\mathrm{C}_{8} \mathrm{H}_{7} \mathrm{Br}_{1}{ }^{+}[\mathrm{M}]^{+}, 181.9727$; found, 181.9726; deviation: -0.6 ppm. 


\section{4-Vinylbenzaldehyde (34)}

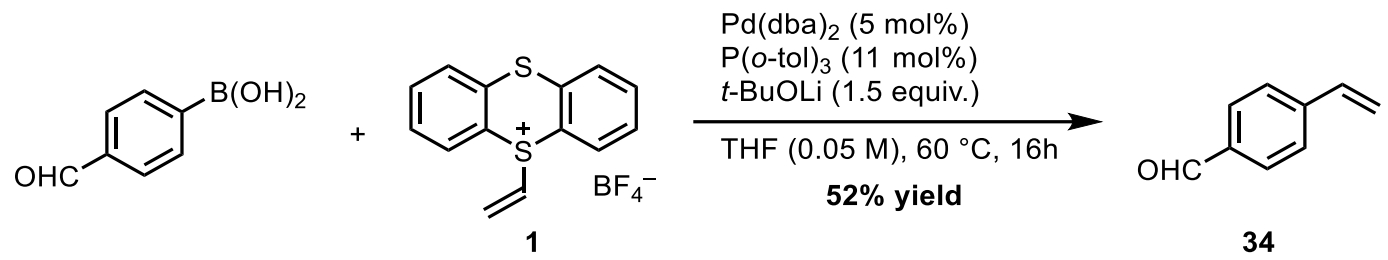

The title compound was prepared following general procedure B. Under ambient atmosphere, a $20 \mathrm{~mL}$ vial equipped with a teflon-coated magnetic stirring bar was charged with 4-formylphenylboronic acid (45.0 mg, $0.300 \mathrm{mmol}, 1.00$ equiv.), Pd(dba)2 (8.6 mg, $15 \mu \mathrm{mol}, 5.0 \mathrm{~mol} \%), \mathrm{P}(\mathrm{o} \text {-tol })_{3}(10.0 \mathrm{mg}, 33.0 \mu \mathrm{mol}, 11.0 \mathrm{~mol} \%)$ and $t$-BuOLi (36.0 mg, $0.450 \mathrm{mmol}, 1.50$ equiv.). The vial was transferred into a $\mathrm{N}_{2}$-filled glove box. Subsequently, dry THF $(6 \mathrm{~mL}, \mathrm{c}=0.05 \mathrm{M})$ was added into the vial. The reaction mixture was stirred for $2 \mathrm{~min}$ at $25^{\circ} \mathrm{C}$ before 1 (149 mg, $0.450 \mathrm{mmol}, 1.50$ equiv.) was added into the vial in one portion. The vial was capped and was then transferred out of the glove box. The vial was placed on a heating block preheated at $60{ }^{\circ} \mathrm{C}$ where the reaction mixture was stirred for $16 \mathrm{~h}$. The reaction mixture was cooled to $25^{\circ} \mathrm{C}$ and filtered through a pad of celite eluting with DCM $(20 \mathrm{~mL})$. The filtrate was collected and concentrated under vacuum to roughly $5 \mathrm{~mL}$. Silica gel (approximately $300 \mathrm{mg}$ ) was added, and the mixture was evaporated to dryness under reduced pressure. The residue was purified by column chromatography on silica gel eluting with a solvent mixture of EtOAc:pentane $(1: 50,(\mathrm{v}: \mathrm{v}))$ to afford $21.0 \mathrm{mg}$ of the title compound (34) as a colorless oil (52\% yield).

$\mathbf{R} \boldsymbol{f}=0.29($ EtOAc:pentane, 1:19 (v:v)).

\section{NMR Spectroscopy:}

${ }^{1} \mathrm{H}$ NMR $\left(500 \mathrm{MHz}, \mathrm{CDCl}_{3}, 298 \mathrm{~K}, \delta\right): 9.99(\mathrm{~s}, 1 \mathrm{H}), 8.05-7.78(\mathrm{~m}, 2 \mathrm{H}), 7.58-7.52(\mathrm{~m}, 2 \mathrm{H}), 6.77$ (dd, J $=17.6,10.9 \mathrm{~Hz}, 1 \mathrm{H}), 5.91(\mathrm{dd}, J=17.6,0.6 \mathrm{~Hz}, 1 \mathrm{H}), 5.44(\mathrm{dd}, J=10.9,0.6 \mathrm{~Hz}, 1 \mathrm{H}) \mathrm{ppm}$.

${ }^{13} \mathrm{C}$ NMR $\left(75 \mathrm{MHz}, \mathrm{CDCl}_{3}, 298 \mathrm{~K}, \delta\right): 191.86,143.62,136.06,135.85,130.25,126.90,117.61$ ppm.

HRMS-EI (m/z) calculated for $\mathrm{C}_{9} \mathrm{H}_{8} \mathrm{O}_{1}{ }^{+}[\mathrm{M}]^{+}, 132.0571$; found, 132.0570; deviation: -0.9 ppm.

\section{2-Vinylbenzo[b]thiophene (35)}

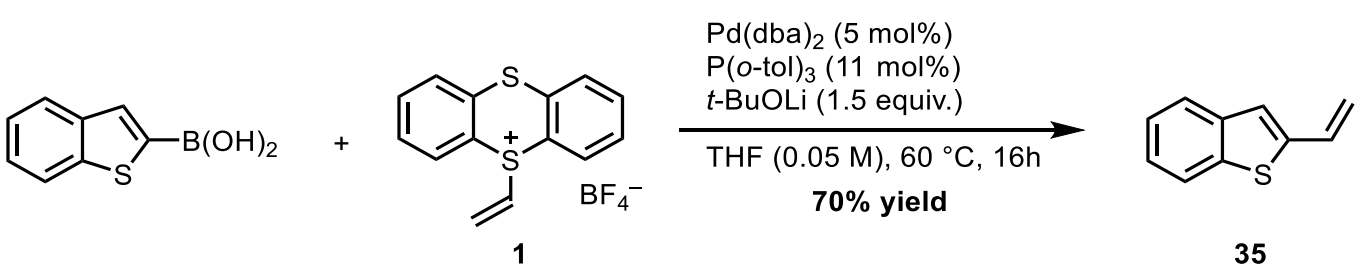

The title compound was prepared following general procedure B. Under ambient atmosphere, a $20 \mathrm{~mL}$ vial equipped with a teflon-coated magnetic stirring bar was charged with benzo[b]thien-2-ylboronic acid (53.4 mg, 0.300 mmol, 1.00 equiv.), Pd(dba) 2 ( $8.6 \mathrm{mg}, 15 \mu \mathrm{mol}, 5.0 \mathrm{~mol} \%$ ), P(o-tol) 3 (10.0 mg, $33.0 \mu \mathrm{mol}$, $11.0 \mathrm{~mol} \%)$ and $t$-BuOLi (36.0 mg, $0.450 \mathrm{mmol}, 1.50$ equiv.). The vial was transferred into a $\mathrm{N}_{2}$-filled glove 
box. Subsequently, dry THF ( $6 \mathrm{~mL}, \mathrm{c}=0.05 \mathrm{M})$ was added into the vial. The reaction mixture was stirred for 2 min at $25^{\circ} \mathrm{C}$ before 1 (149 mg, $0.450 \mathrm{mmol}, 1.50$ equiv.) was added into the vial in one portion. The vial was capped and was then transferred out of the glove box. The vial was placed on a heating block preheated at $60{ }^{\circ} \mathrm{C}$ where the reaction mixture was stirred for $16 \mathrm{~h}$. The reaction mixture was cooled to $25^{\circ} \mathrm{C}$ and filtered through a pad of celite eluting with DCM $(20 \mathrm{~mL})$. The filtrate was collected and concentrated under vacuum to roughly $5 \mathrm{~mL}$. Silica gel (approximately $300 \mathrm{mg}$ ) was added, and the mixture was evaporated to dryness under reduced pressure. The residue was purified by column chromatography on silica gel eluting with pentane to afford $33.6 \mathrm{mg}$ of the title compound (35) as a white solid (70\% yield).

$\mathbf{R} \boldsymbol{f}=0.29$ (pentane).

\section{NMR Spectroscopy:}

${ }^{1} \mathrm{H}$ NMR $\left(500 \mathrm{MHz}, \mathrm{CDCl}_{3}, 298 \mathrm{~K}, \delta\right): 7.86-7.74(\mathrm{~m}, 1 \mathrm{H}), 7.74-7.59(\mathrm{~m}, 1 \mathrm{H}), 7.41-7.27(\mathrm{~m}, 2 \mathrm{H}), 7.18$ (s, 1H), 6.93 (ddd, $J=17.2,10.7,0.6 \mathrm{~Hz}, 1 \mathrm{H}), 5.68(\mathrm{~d}, J=17.3 \mathrm{~Hz}, 1 \mathrm{H}), 5.32(\mathrm{~d}, J=10.7 \mathrm{~Hz}, 1 \mathrm{H}) \mathrm{ppm}$.

${ }^{13} \mathrm{C}$ NMR $\left(126 \mathrm{MHz}, \mathrm{CDCl}_{3}, 298 \mathrm{~K}, \delta\right): 143.2,140.1,139.0,130.7,124.9,124.5,123.7,123.2,122.4$, $116.1 \mathrm{ppm}$.

HRMS-El (m/z) calculated for $\mathrm{C}_{10} \mathrm{H}_{8} \mathrm{~S}_{1}{ }^{+}[\mathrm{M}]^{+}, 160.0341$; found, 160.0341 ; deviation: $+0.1 \mathrm{ppm}$.

1-Methyl-3-(trifluoromethyl)-5-vinyl-1H-pyrazole (36)

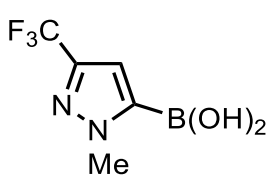

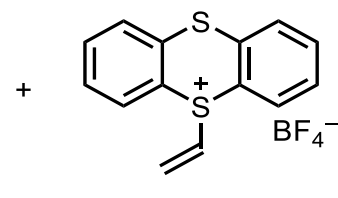

1

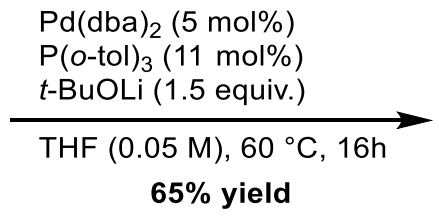

$65 \%$ yield

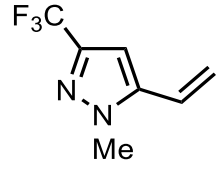

36

The title compound was prepared following general procedure B. Under ambient atmosphere, a $20 \mathrm{~mL}$ vial equipped with a teflon-coated magnetic stirring bar was charged with (1-methyl-3-(trifluoromethyl)-1Hpyrazol-5-yl)boronic acid (58.2 mg, 0.300 mmol, 1.00 equiv.), $\mathrm{Pd}(\mathrm{dba})_{2}(8.6 \mathrm{mg}, 15 \mu \mathrm{mol}, 5.0 \mathrm{~mol} \%), \mathrm{P}(\mathrm{o}$-tol) 3 $(10.0 \mathrm{mg}, 33.0 \mu \mathrm{mol}, 11.0 \mathrm{~mol} \%)$ and $t$-BuOLi (36.0 mg, $0.450 \mathrm{mmol}, 1.50$ equiv.). The vial was transferred into a $\mathrm{N}_{2}$-filled glove box. Subsequently, dry THF (6 mL, C $\left.=0.05 \mathrm{M}\right)$ was added into the vial. The reaction mixture was stirred for $2 \mathrm{~min}$ at $25^{\circ} \mathrm{C}$ before 1 (149 mg, $0.450 \mathrm{mmol}, 1.50$ equiv.) was added into the vial in one portion. The vial was capped and was then transferred out of the glove box. The vial was placed on a heating block preheated at $60{ }^{\circ} \mathrm{C}$ where the reaction mixture was stirred for $16 \mathrm{~h}$. The reaction mixture was cooled to $25^{\circ} \mathrm{C}$ and filtered through a pad of celite eluting with DCM $(20 \mathrm{~mL})$. The filtrate was collected and concentrated under vacuum to roughly $5 \mathrm{~mL}$. Silica gel (approximately $300 \mathrm{mg}$ ) was added, and the mixture was evaporated to dryness under reduced pressure at temperature $<30^{\circ} \mathrm{C}$. The residue was purified by column chromatography on silica gel eluting with a solvent mixture of DCM:pentane $(1: 2,(\mathrm{v}: \mathrm{v}))$ to afford $34.3 \mathrm{mg}$ of the title compound (36) as a colorless oil (65\% yield). [Note: due to the volatility of the product, drying of the purified product was processed under vacuum in a bath of dry ice]. 
$\mathbf{R}_{\boldsymbol{f}}=0.30$ (DCM:pentane, $\left.1: 1(\mathrm{v}: \mathrm{v})\right)$.

\section{NMR Spectroscopy:}

${ }^{1} \mathrm{H}$ NMR $\left(500 \mathrm{MHz}, \mathrm{CDCl}_{3}, 298 \mathrm{~K}, \delta\right): 6.62(\mathrm{~s}, 1 \mathrm{H}), 6.58$ (dd, $\left.J=17.5,11.2 \mathrm{~Hz}, 1 \mathrm{H}\right), 5.76$ (dd, $J=17.4$, $1.0 \mathrm{~Hz}, 1 \mathrm{H}), 5.57-5.35(\mathrm{~m}, 1 \mathrm{H}), 3.91(\mathrm{~s}, 3 \mathrm{H}) \mathrm{ppm}$.

${ }^{13} \mathrm{C}$ NMR (151 MHz, $\left.\mathrm{CDCl}_{3}, 298 \mathrm{~K}, \delta\right): 142.6,141.6$ (q, $\left.J=38.0 \mathrm{~Hz}\right), 122.7,121.4$ (q, $\left.J=268.4 \mathrm{~Hz}\right)$, 119.8, 101.6 (q, $J=2.3 \mathrm{~Hz}$ ), $37.4 \mathrm{ppm}$.

${ }^{19} \mathrm{~F} \mathrm{NMR}\left(471 \mathrm{MHz}, \mathrm{CDCl}_{3}, 298 \mathrm{~K}, \delta\right):-62.23 \mathrm{ppm}$.

HRMS-EI (m/z) calculated for $\mathrm{C}_{7} \mathrm{H}_{7} \mathrm{~N}_{2} \mathrm{~F}_{3}{ }^{+}[\mathrm{M}]^{+}, 176.0556$; found, 176.0556; deviation: 0.2 ppm.

1-Chloro-4-(trifluoromethyl)-2-((2-vinylbenzyl)oxy)benzene (37)<smiles>Oc1ccccc1COc1cc(C(F)(F)F)ccc1Cl</smiles><smiles>C=C[S+]1c2ccccc2Sc2ccccc21</smiles>

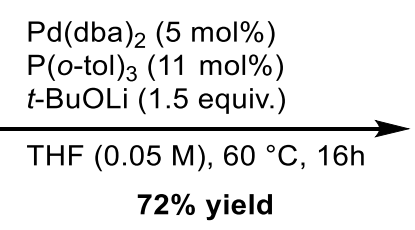

$72 \%$ yield

1

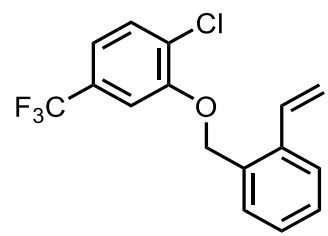

37

The title compound was prepared following general procedure B. Under ambient atmosphere, a $20 \mathrm{~mL}$ vial equipped with a teflon-coated magnetic stirring bar was charged with 2-((2'-Chloro-5'-

(trifluoromethyl)phenoxy)methyl)phenylboronic acid (99.1 mg, $0.300 \mathrm{mmol}, 1.00$ equiv.), Pd(dba)2 (8.6 mg, $15 \mu \mathrm{mol}, 5.0 \mathrm{~mol} \%), \mathrm{P}(o$-tol) $3(10.0 \mathrm{mg}, 33.0 \mu \mathrm{mol}, 11.0 \mathrm{~mol} \%)$ and $t$-BuOLi (36.0 mg, $0.450 \mathrm{mmol}$, 1.50 equiv.). The vial was transferred into a $\mathrm{N}_{2}$-filled glove box. Subsequently, dry THF $(6 \mathrm{~mL}, \mathrm{C}=0.05 \mathrm{M})$ was added into the vial. The reaction mixture was stirred for $2 \mathrm{~min}$ at $25^{\circ} \mathrm{C}$ before $1(149 \mathrm{mg}, 0.450 \mathrm{mmol}$, 1.50 equiv.) was added into the vial in one portion. The vial was capped and was then transferred out of the glove box. The vial was placed on a heating block preheated at $60^{\circ} \mathrm{C}$ where the reaction mixture was stirred for $16 \mathrm{~h}$. The reaction mixture was cooled to $25^{\circ} \mathrm{C}$ and filtered through a pad of celite eluting with DCM (20 $\mathrm{mL}$ ). The filtrate was collected and concentrated under vacuum to roughly $5 \mathrm{~mL}$. Silica gel (approximately $300 \mathrm{mg}$ ) was added, and the mixture was evaporated to dryness under reduced pressure. The residue was purified by column chromatography on silica gel eluting with pentane to afford $67.4 \mathrm{mg}$ of the title compound (37) as a white solid (72\% yield).

$\mathbf{R}_{\boldsymbol{f}}=0.51$ (EtOAc:pentane, 1:19 (v:v)).

\section{NMR Spectroscopy:}

${ }^{1} \mathrm{H}$ NMR $\left(500 \mathrm{MHz}, \mathrm{CDCl}_{3}, 298 \mathrm{~K}, \delta\right): 7.57$ (dd, $\left.J=7.6,1.5 \mathrm{~Hz}, 1 \mathrm{H}\right), 7.50$ (ddd, $J=8.0,6.0,1.2 \mathrm{~Hz}, 2 \mathrm{H}$ ), 7.37 (td, $J=7.5,1.6 \mathrm{~Hz}, 1 \mathrm{H}$ ), 7.32 (td, $J=7.5,1.5 \mathrm{~Hz}, 1 \mathrm{H}$ ), $7.23(\mathrm{~m}, 1 \mathrm{H}), 7.19$ (ddd, $J=8.2,1.9,0.8 \mathrm{~Hz}$, 1H), 7.03 (dd, $J=17.3,11.0 \mathrm{~Hz}, 1 \mathrm{H}$ ), 5.71 (dd, $J=17.4,1.3 \mathrm{~Hz}, 1 \mathrm{H}), 5.40$ (dd, $J=11.0,1.2 \mathrm{~Hz}, 1 \mathrm{H}$ ), $5.21(\mathrm{~s}, 2 \mathrm{H}) \mathrm{ppm}$.

${ }^{13} \mathrm{C}$ NMR (126 MHz, $\left.\mathrm{CDCl}_{3}, 298 \mathrm{~K}, \delta\right): 154.4,137.3,133.6,132.3,130.7,129.9$ (q, J = $\left.32.8 \mathrm{~Hz}\right), 128.9$, 
128.8, 128.0, 127.4, 126.3, 123.6 (q, $J=272.4 \mathrm{~Hz}), 118.5$ (q, $J=3.9 \mathrm{~Hz}), 117.3,110.6$ (q, $J=3.7 \mathrm{~Hz})$, $69.4 \mathrm{ppm}$.

${ }^{19}$ F NMR $\left(471 \mathrm{MHz}, \mathrm{CDCl}_{3}, 298 \mathrm{~K}, \delta\right):-62.50 \mathrm{ppm}$.

HRMS-EI (m/z) calculated for $\mathrm{C}_{16} \mathrm{H}_{12} \mathrm{O}_{1} \mathrm{Na}_{1} \mathrm{~F}_{3} \mathrm{Cl}_{1}{ }^{+}[\mathrm{M}+\mathrm{Na}]^{+}$, 335.0421; found, 335.0421; deviation: $-0.1 \mathrm{ppm}$.

Piperidin-1-yl(4-vinylphenyl)methanone (38)
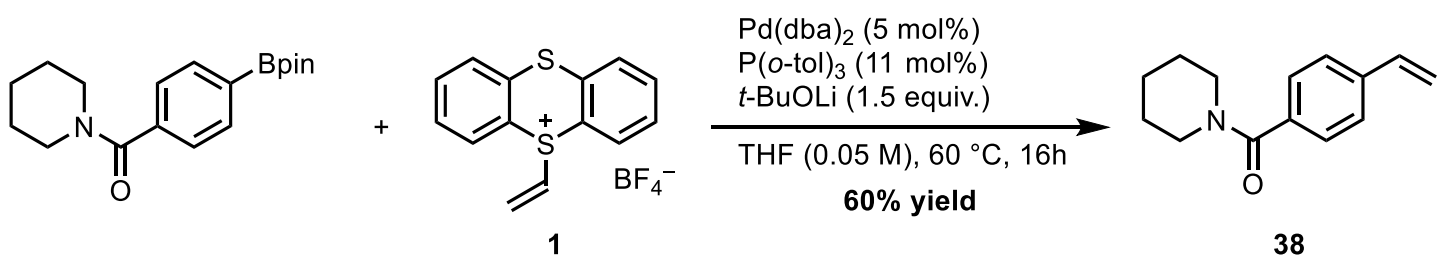

The title compound was prepared following general procedure B. Under ambient atmosphere, a $20 \mathrm{~mL}$ vial equipped with a teflon-coated magnetic stirring bar was charged with 4-(piperidine-1-carbonyl)phenylboronic acid pinacol ester (94.6 mg, $0.300 \mathrm{mmol}, 1.00$ equiv.), $\mathrm{Pd}(\mathrm{dba})_{2}$ (8.6 mg, $\left.15 \mu \mathrm{mol}, 5.0 \mathrm{~mol} \%\right), \mathrm{P}(\mathrm{o}$-tol) 3 $(10.0 \mathrm{mg}, 33.0 \mu \mathrm{mol}, 11.0 \mathrm{~mol} \%)$ and $t$-BuOLi $(36.0 \mathrm{mg}, 0.450 \mathrm{mmol}, 1.50$ equiv.). The vial was transferred into a $\mathrm{N}_{2}$-filled glove box. Subsequently, dry THF $(6 \mathrm{~mL}, \mathrm{C}=0.05 \mathrm{M})$ was added into the vial. The reaction mixture was stirred for $2 \mathrm{~min}$ at $25^{\circ} \mathrm{C}$ before 1 (149 mg, $0.450 \mathrm{mmol}, 1.50$ equiv.) was added into the vial in one portion. The vial was capped and was then transferred out of the glove box. The vial was placed on a heating block preheated at $60^{\circ} \mathrm{C}$ where the reaction mixture was stirred for $16 \mathrm{~h}$. The reaction mixture was cooled to $25^{\circ} \mathrm{C}$ and filtered through a pad of celite eluting with DCM $(20 \mathrm{~mL})$. The filtrate was collected and concentrated under vacuum to roughly $5 \mathrm{~mL}$. Silica gel (approximately $300 \mathrm{mg}$ ) was added, and the mixture was evaporated to dryness under reduced pressure. The residue was purified by column chromatography on silica gel eluting with a solvent mixture of EtOAc:pentane $(1: 4(\mathrm{v}: \mathrm{v}))$ to afford $38.8 \mathrm{mg}$ of the title compound (38) as a white solid (60\% yield).

$\mathbf{R} \boldsymbol{f}=0.40$ (EtOAc:pentane, 1:1 (v:v)).

\section{NMR Spectroscopy:}

${ }^{1} \mathrm{H}$ NMR $\left(500 \mathrm{MHz}, \mathrm{CDCl}_{3}, 298 \mathrm{~K}, \delta\right): 7.47-7.39(\mathrm{~m}, 2 \mathrm{H}), 7.37-7.30(\mathrm{~m}, 2 \mathrm{H}), 6.72(\mathrm{dd}, J=17.6$, $10.9 \mathrm{~Hz}, 1 \mathrm{H}$ ), 5.79 (dd, $J=17.6,0.8 \mathrm{~Hz}, 1 \mathrm{H}), 5.30$ (dd, $J=10.8,0.6 \mathrm{~Hz}, 1 \mathrm{H}), 3.75-3.60(\mathrm{~m}, 2 \mathrm{H}), 3.44-$ $3.27(\mathrm{~m}, 2 \mathrm{H}), 1.74-1.62(\mathrm{~m}, 4 \mathrm{H}), 1.55-1.42(\mathrm{~m}, 2 \mathrm{H}) \mathrm{ppm}$.

${ }^{13} \mathrm{C}$ NMR (126 MHz, $\left.\mathrm{CD}_{3} \mathrm{CN}, 243 \mathrm{~K}, \delta\right): 169.6,138.6,136.6,136.4,127.6,126.5,115.3,48.8,42.9,26.5$, 25.8, 24.7 .

HRMS-EI (m/z) calculated for $\mathrm{C}_{14} \mathrm{H}_{17} \mathrm{~N}_{1} \mathrm{O}_{1}{ }^{+}[\mathrm{M}]^{+}, 215.1303$; found, 215.1305; deviation: -0.9 ppm. 
Morpholino(3-vinylphenyl)methanone (39)<smiles>C=C[S+]1c2ccccc2Sc2ccccc21</smiles>

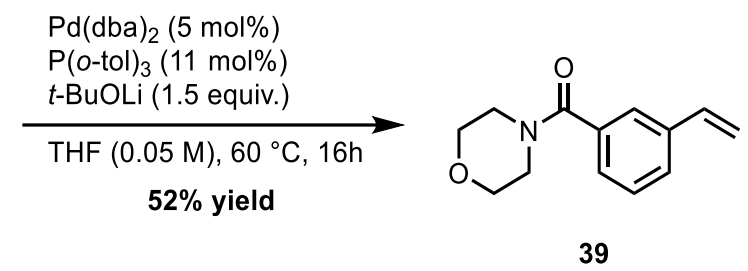

The title compound was prepared following general procedure B. Under ambient atmosphere, a $20 \mathrm{~mL}$ vial equipped with a teflon-coated magnetic stirring bar was charged with potassium 3-(4morpholinylcarbonyl)phenyltrifluoroborate $(89.1 \mathrm{mg}, 0.300 \mathrm{mmol}, 1.00$ equiv.), $\mathrm{Pd}(\mathrm{dba}) 2$ (8.6 mg, $15 \mu \mathrm{mol}$, $5.0 \mathrm{~mol} \%), \mathrm{P}(o$-tol) $3(10.0 \mathrm{mg}, 33.0 \mu \mathrm{mol}, 11.0 \mathrm{~mol} \%)$ and $t$-BuOLi (36.0 mg, $0.450 \mathrm{mmol}, 1.50$ equiv.). The vial was transferred into a $\mathrm{N}_{2}$-filled glove box. Subsequently, dry THF $(6 \mathrm{~mL}, \mathrm{c}=0.05 \mathrm{M})$ was added into the vial. The reaction mixture was stirred for $2 \mathrm{~min}$ at $25^{\circ} \mathrm{C}$ before 1 (149 mg, $0.450 \mathrm{mmol}, 1.50$ equiv.) was added into the vial in one portion. The vial was capped and was then transferred out of the glove box. The vial was placed on a heating block preheated at $60^{\circ} \mathrm{C}$ where the reaction mixture was stirred for $16 \mathrm{~h}$. The reaction mixture was cooled to $25^{\circ} \mathrm{C}$ and filtered through a pad of celite eluting with DCM $(20 \mathrm{~mL})$. The filtrate was collected and concentrated under vacuum to roughly $5 \mathrm{~mL}$. Silica gel (approximately $300 \mathrm{mg}$ ) was added, and the mixture was evaporated to dryness under reduced pressure. The residue was purified by column chromatography on silica gel eluting with pentane to afford $34.3 \mathrm{mg}$ of the title compound (39) as a colorless oil (52\% yield).

$\mathbf{R} \boldsymbol{f}=0.23$ (EtOAc:pentane, v:v (1:1)).

\section{NMR Spectroscopy:}

${ }^{1} \mathrm{H}$ NMR $\left(500 \mathrm{MHz}, \mathrm{CDCl}_{3}, 298 \mathrm{~K}, \delta\right): 7.54-7.43(\mathrm{~m}, 2 \mathrm{H}), 7.36$ (t, $\left.J=7.6 \mathrm{~Hz}, 1 \mathrm{H}\right), 7.26(\mathrm{dt}, J=7.6$, $1.4 \mathrm{~Hz}, 1 \mathrm{H}), 6.71(\mathrm{dd}, J=17.6,10.9 \mathrm{~Hz}, 1 \mathrm{H}), 5.78(\mathrm{~d}, J=17.6 \mathrm{~Hz}, 1 \mathrm{H}), 5.31(\mathrm{~d}, J=10.9 \mathrm{~Hz}, 1 \mathrm{H}), 3.79-$ $3.43(\mathrm{~m}, 8 \mathrm{H}) \mathrm{ppm}$.

${ }^{13} \mathrm{C}$ NMR $\left(126 \mathrm{MHz}, \mathrm{CDCl}_{3}, 298 \mathrm{~K}, \delta\right): 170.4,138.2,136.1,135.8,128.8,127.7,126.3,125.0,115.3$, $67.0 \mathrm{ppm}$.

HRMS-El (m/z) calculated for $\mathrm{C}_{13} \mathrm{H}_{15} \mathrm{~N}_{1} \mathrm{O}_{2} \mathrm{Na}_{1}{ }^{+}[\mathrm{M}+\mathrm{Na}]^{+}, 240.0997$; found, 240.0995; deviation: -1.0 ppm.

(E)-4-(Buta-1,3-dien-1-yl)-1,1'-biphenyl (40)
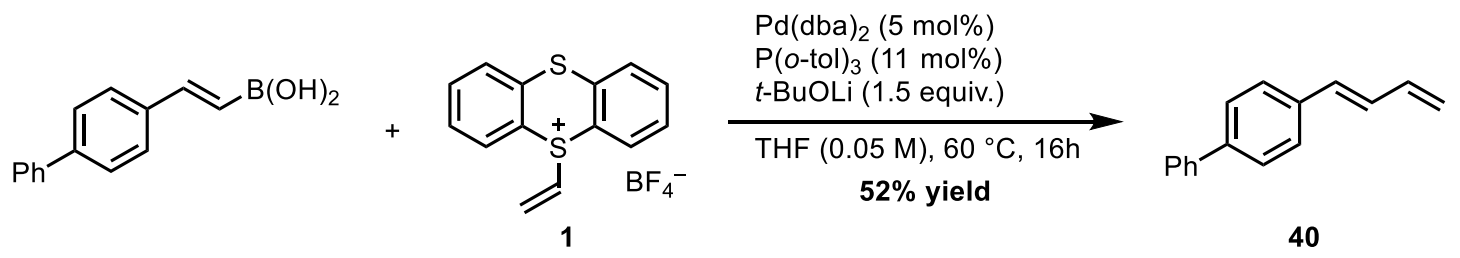

The title compound was prepared following general procedure B. Under ambient atmosphere, a $20 \mathrm{~mL}$ vial equipped with a teflon-coated magnetic stirring bar was charged with trans-2-(4-Biphenyl)vinylboronic acid 
(67.2 mg, 0.300 mmol, 1.00 equiv.), Pd(dba) 2 (8.6 mg, $15 \mu \mathrm{mol}, 5.0 \mathrm{~mol} \%$ ), P(o-tol) 3 (10.0 mg, $33.0 \mu \mathrm{mol}$, $11.0 \mathrm{~mol} \%$ ) and $t$-BuOLi (36.0 mg, $0.450 \mathrm{mmol}, 1.50$ equiv.). The vial was transferred into a $\mathrm{N}_{2}$-filled glove box. Subsequently, dry THF ( $6 \mathrm{~mL}, \mathrm{c}=0.05 \mathrm{M})$ was added into the vial. The reaction mixture was stirred for 2 min at $25^{\circ} \mathrm{C}$ before 1 (149 $\mathrm{mg}, 0.450 \mathrm{mmol}, 1.50$ equiv.) was added into the vial in one portion. The vial was capped and was then transferred out of the glove box. The vial was placed on a heating block preheated at $60{ }^{\circ} \mathrm{C}$ where the reaction mixture was stirred for $16 \mathrm{~h}$. The reaction mixture was cooled to $25^{\circ} \mathrm{C}$ and filtered through a pad of celite eluting with DCM $(20 \mathrm{~mL})$. The filtrate was collected and concentrated under vacuum to roughly $5 \mathrm{~mL}$. Silica gel (approximately $300 \mathrm{mg}$ ) was added, and the mixture was evaporated to dryness under reduced pressure. The residue was purified by column chromatography on silica gel eluting with pentane to afford $32.0 \mathrm{mg}$ of the title compound (40) as a white solid (52\% yield).

$\mathbf{R} \boldsymbol{f}=0.20$ (pentane).

\section{NMR Spectroscopy:}

${ }^{1} \mathrm{H}$ NMR $\left(500 \mathrm{MHz}, \mathrm{CDCl}_{3}, 298 \mathrm{~K}, \delta\right): 7.64-7.54(\mathrm{~m}, 4 \mathrm{H}), 7.51-7.42(\mathrm{~m}, 4 \mathrm{H}), 7.38-7.32(\mathrm{~m}, 1 \mathrm{H}), 6.91$ $-6.78(\mathrm{~m}, 1 \mathrm{H}), 6.67-6.44(\mathrm{~m}, 2 \mathrm{H}), 5.36(\mathrm{dd}, J=17.0,1.4 \mathrm{~Hz}, 1 \mathrm{H}), 5.20(\mathrm{dd}, J=10.1,1.5 \mathrm{~Hz}, 1 \mathrm{H}) \mathrm{ppm}$.

${ }^{13} \mathrm{C} \mathrm{NMR}\left(126 \mathrm{MHz}, \mathrm{CDCl}_{3}, 298 \mathrm{~K}, \delta\right): 140.8,140.5,137.4,136.3,132.5,129.8,128.9,127.5,127.4$, 127.1, 127.0, 117.9 ppm.

HRMS-EI (m/z) calculated for $\mathrm{C}_{16} \mathrm{H}_{14^{+}}[\mathrm{M}]^{+}, 206.1086$; found, 206.1090; deviation: $2.1 \mathrm{ppm}$.

\section{Competition experiment between 1- $d_{3}$ and vinyl bromide.}

\section{Preparation of vinyl bromide solution in THF}

A pre-weighed $10 \mathrm{~mL}$ argon-filled Schlenk tube with rubber septum was cooled down and kept at $-40^{\circ} \mathrm{C}$. To the Schlenk tube was filled with vinyl bromide through a needle from a vinyl bromide cylinder and liquid vinyl bromide condensed at the bottom of the tube. The whole tube was weighed again and the weight of vinyl bromide was the difference between two weights (in this case, $628 \mathrm{mg}, 5.88 \mathrm{mmol}$ ). Then an argon-filled balloon was attached to the tube to balance pressure. THF $(2.0 \mathrm{~mL})$ was added into the tube via syringe to obtain a solution of vinyl bromide (2.9 M).

\section{Competition experiment}

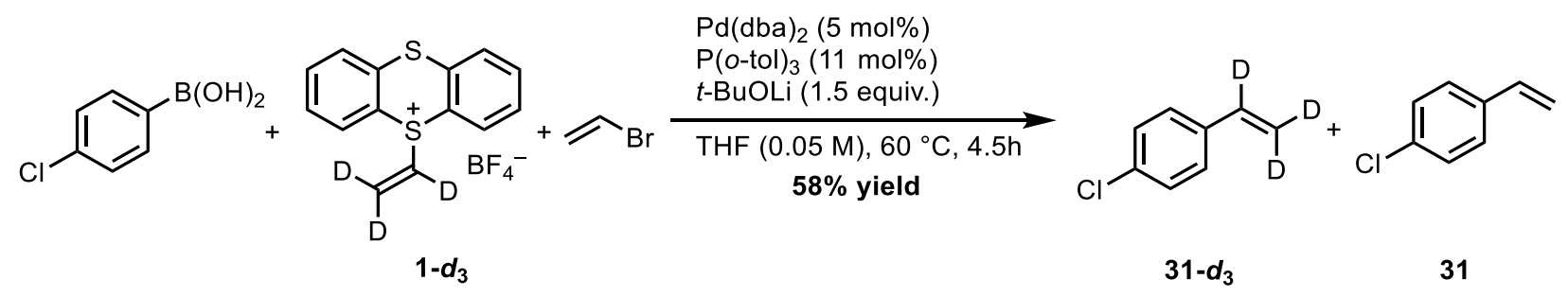

Under ambient atmosphere, a $4 \mathrm{~mL}$ vial equipped with a teflon-coated magnetic stirring bar was charged with 4-chlorophenylboronic acid (7.8 mg, 0.050 mmol, 1.0 equiv.), Pd(dba)2 (1.4 mg, 2.5 mol, 5.0 mol\%), P(o-tol)3 
(1.7 mg, $5.5 \mu \mathrm{mol}, 11 \mathrm{~mol} \%$ ) and $t$-BuOLi $(6.0 \mathrm{mg}, 0.075 \mathrm{mmol}, 1.5$ equiv.). The vial was transferred into a $\mathrm{N}_{2}$-filled glove box. Subsequently, dry THF $(0.82 \mathrm{~mL})$ was added into the vial. The reaction mixture was stirred for $2 \mathrm{~min}$ at $25^{\circ} \mathrm{C}$ before $1-\boldsymbol{d}_{3}(167 \mathrm{mg}, 0.500 \mathrm{mmol}, 10.0$ equiv.) was added into the vial in one portion. The vial was capped and was then transferred out of the glove box. A solution of vinyl bromide in THF (0.18 mL, $2.9 \mathrm{M}, 0.52 \mathrm{mmol}, 10$ equiv.) was added into the vial via syringe. The vial was placed on a heating block preheated at $60{ }^{\circ} \mathrm{C}$ where the reaction mixture was stirred for $4.5 \mathrm{~h}$. The reaction mixture was cooled to $25^{\circ} \mathrm{C}$ and filtered through a pad of celite eluting with DCM $(10 \mathrm{~mL})$. Silica gel (approximately $50 \mathrm{mg}$ ) was added to the filtrate, and the mixture was evaporated to dryness under reduced pressure. The residue was purified by column chromatography on silica gel eluting with pentane to afford $3.2 \mathrm{mg}$ of a

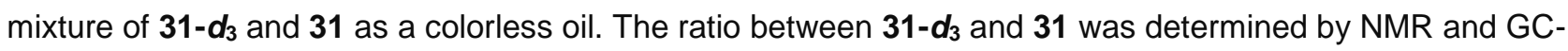
HRMS analysis.

In the ${ }^{1} \mathrm{H}$ NMR spectrum of the mixture, the vinyl protons of $\mathbf{3 1}$ cannot be observed (Figure S10).

Deuterium incorporation: $>2.99{ }^{2} \mathrm{H} /$ molecule

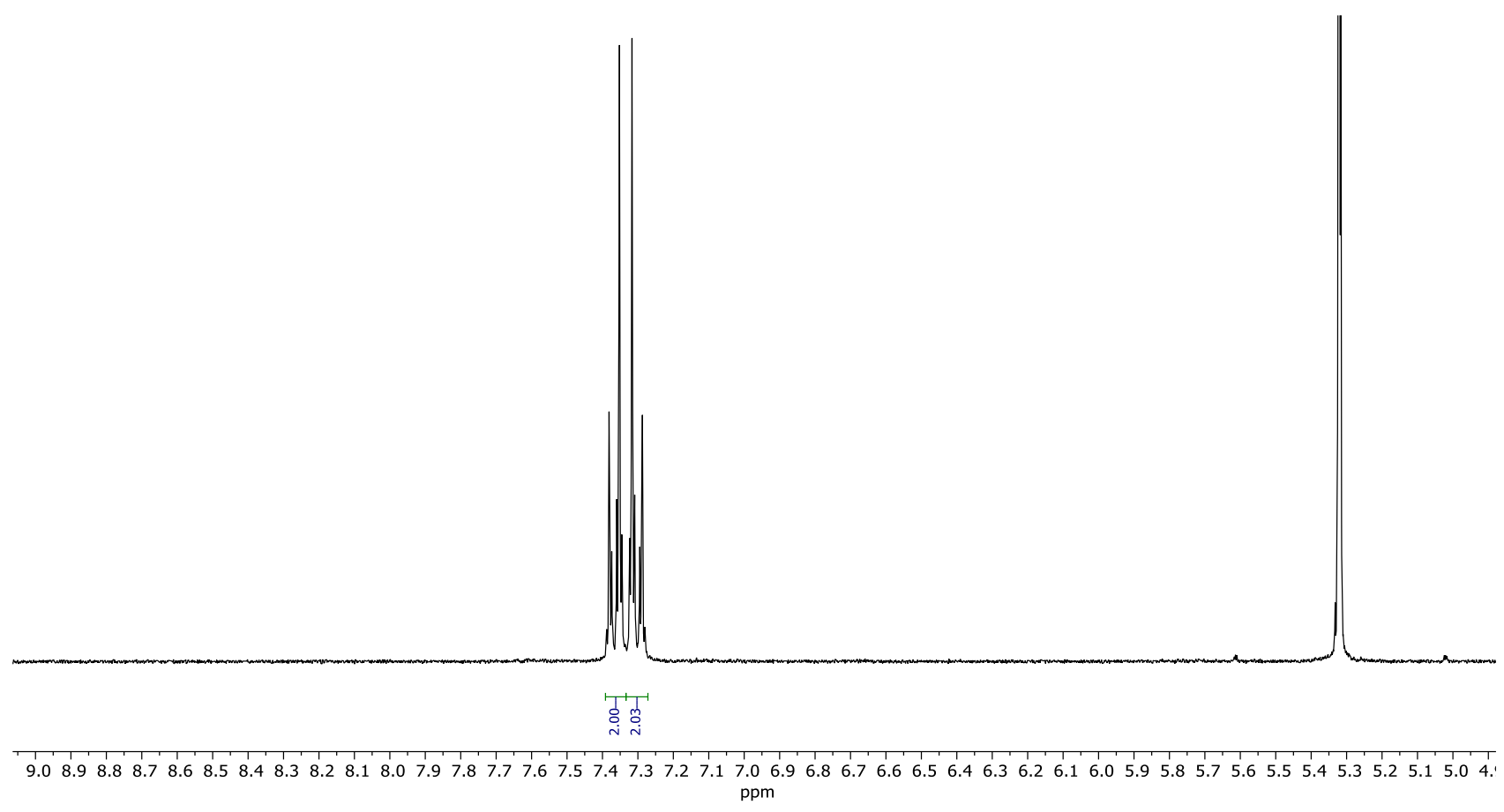

Figure S10. NMR spectrum of a mixture of $\mathbf{3 1}-\boldsymbol{d}_{3}$ and $\mathbf{3 1}, \mathrm{CD}_{2} \mathrm{Cl}_{2}, 300 \mathrm{MHz}, 298 \mathrm{~K}$

Isotopic labeling analysis by HRMS spectrometry shows the mixture contains $99.38 \%$ of $\mathbf{3 1}-\boldsymbol{d}_{\mathbf{n}}(\mathrm{n}=1-3)$ and $0.61 \%$ of $\mathbf{3 1}-d_{0}$ (Figure $S 11$ ). The ratio is $163: 1$. 


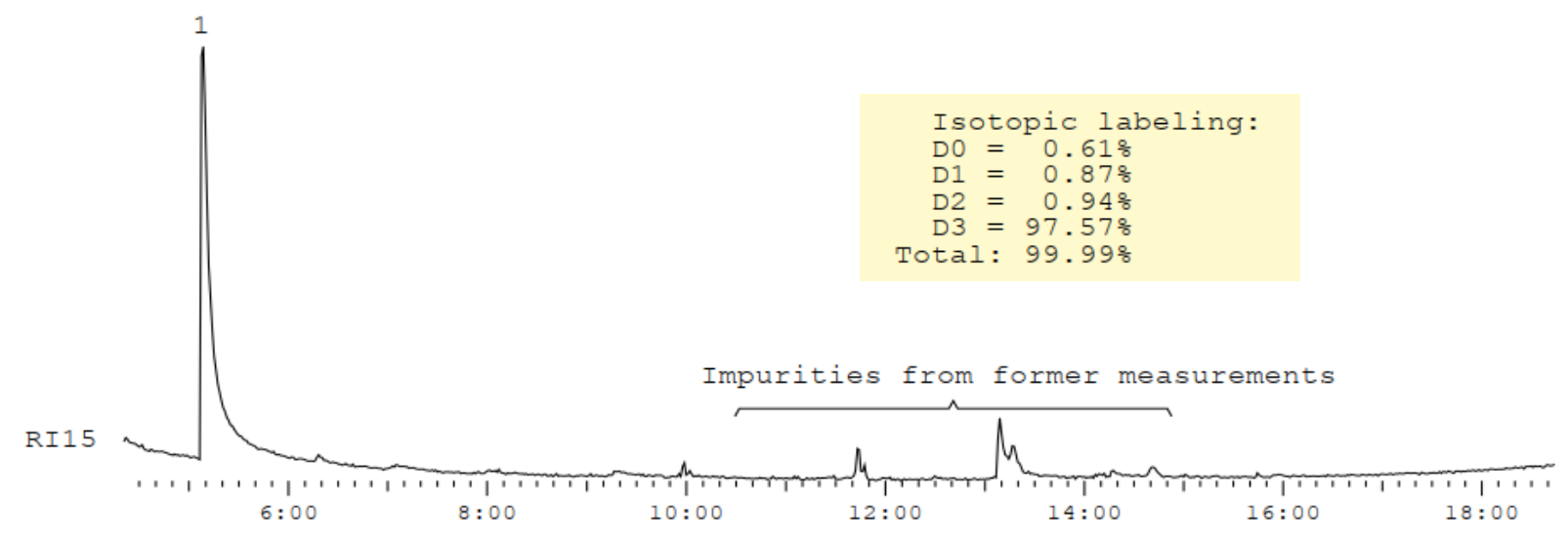

Figure S11. GC-MS isotopic labeling analysis of the mixture.

\section{Comparison on the performance of 1 and vinyl-SPh 2 (OTf) in Suzuki-type reactions.}

General procedure C

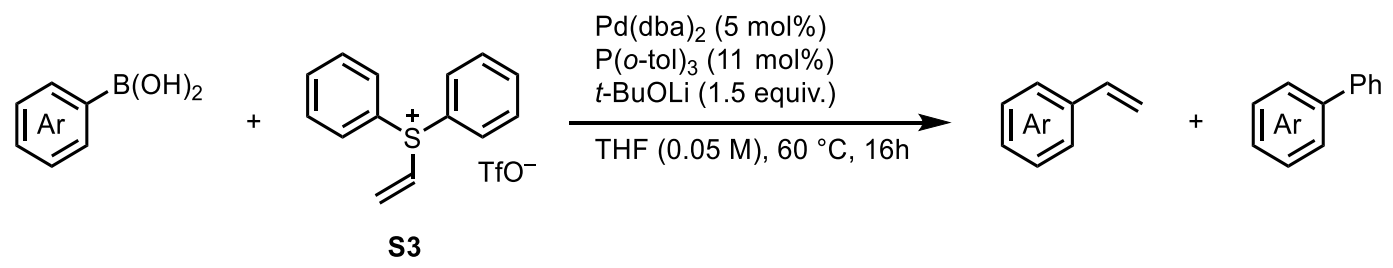

Under ambient atmosphere, a $20 \mathrm{~mL}$ vial equipped with a teflon-coated magnetic stirring bar was charged with aryl boronic acid $(0.300 \mathrm{mmol}, 1.00$ equiv.), Pd(dba)2 (8.6 mg, $15 \mu \mathrm{mol}, 5.0 \mathrm{~mol} \%), \mathrm{P}(\mathrm{o}$-tol)3 (10.0 mg, $33.0 \mu \mathrm{mol}, 11.0 \mathrm{~mol} \%)$ and $t$-BuOLi $\left(36.0 \mathrm{mg}, 0.450 \mathrm{mmol}, 1.50\right.$ equiv.). The vial was transferred into a $\mathrm{N}_{2-}$ filled glove box. Subsequently, dry THF $(4 \mathrm{~mL})$ was added into the vial. The reaction mixture was stirred for 2 min at $25^{\circ} \mathrm{C}$ before a solution of vinylSPh$(\mathrm{OTf})(\mathrm{S} 3,163 \mathrm{mg}, 0.450 \mathrm{mmol}, 1.50$ equiv.) in THF (2 mL) was added into the vial via syringe. The vial was capped and was then transferred out of the glove box. The vial was placed on a heating block preheated at $60^{\circ} \mathrm{C}$ where the reaction mixture was stirred for $16 \mathrm{~h}$. The reaction mixture was cooled to $25^{\circ} \mathrm{C}$ and filtered through a pad of celite eluting with $\mathrm{DCM}(20 \mathrm{~mL})$. The filtrate was collected and concentrated under vacuum to roughly $5 \mathrm{~mL}$. Silica gel (approximately $300 \mathrm{mg}$ ) was added, and the mixture was evaporated to dryness under reduced pressure. The residue was purified by column chromatography on silica gel to give corresponding product. 
tert-Butyl (3-vinylphenyl)carbamate (45)

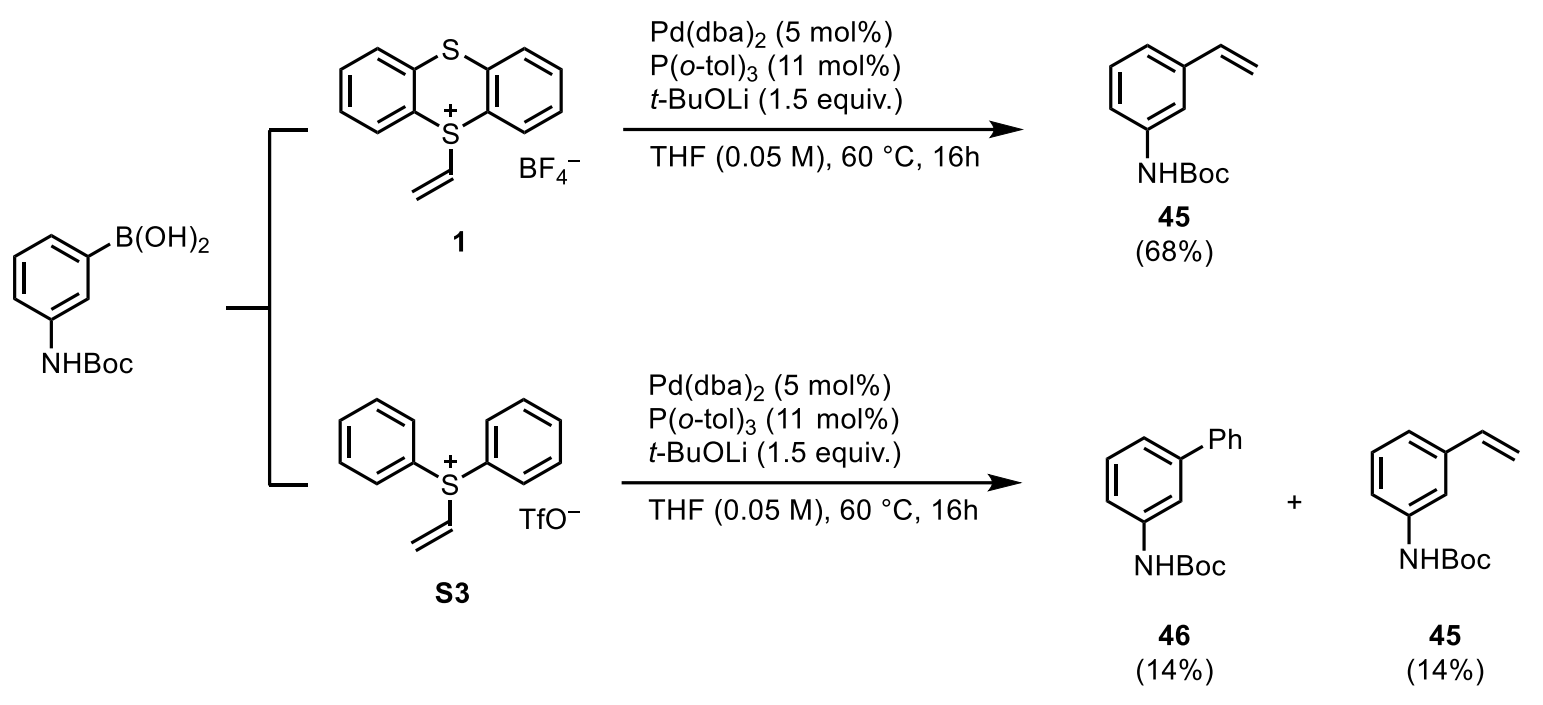

The title compound was prepared following general procedure B (for 1) or C (for S3). Under ambient atmosphere, a $20 \mathrm{~mL}$ vial equipped with a teflon-coated magnetic stirring bar was charged with 3-(N-Bocamino)phenylboronic acid (71.1 mg, $0.300 \mathrm{mmol}, 1.00$ equiv.), $\mathrm{Pd}(\mathrm{dba})_{2}(8.6 \mathrm{mg}, 15 \mu \mathrm{mol}, 5.0 \mathrm{~mol} \%), \mathrm{P}\left(\mathrm{o}^{-}\right.$ tol)3 (10.0 mg, $33.0 \mu \mathrm{mol}, 11.0 \mathrm{~mol} \%$ ) and $t$-BuOLi (36.0 mg, $0.450 \mathrm{mmol}, 1.50$ equiv.). The vial was transferred into a $\mathrm{N}_{2}$-filled glove box. Subsequently, dry THF (4 mL) was added into the vial. The reaction mixture was stirred for $2 \mathrm{~min}$ at $25^{\circ} \mathrm{C}$ before 1 (149 mg, $0.450 \mathrm{mmol}, 1.50$ equiv.) or S3 (163 mg, $0.450 \mathrm{mmol}, 1.50$ equiv; as a solution in $2 \mathrm{~mL}$ of THF) were added into the vial in one portion. The vial was capped and was then transferred out of the glove box. The vial was placed on a heating block preheated at $60{ }^{\circ} \mathrm{C}$ where the reaction mixture was stirred for $16 \mathrm{~h}$. The reaction mixture was cooled to $25^{\circ} \mathrm{C}$ and filtered through a pad of celite eluting with DCM $(20 \mathrm{~mL})$. The filtrate was collected and concentrated under vacuum to roughly $5 \mathrm{~mL}$. Silica gel (approximately $300 \mathrm{mg}$ ) was added, and the mixture was evaporated to dryness under reduced pressure. The residue was purified by column chromatography on silica gel eluting with a solvent mixture of EtOAc:pentane (1:50 (v:v)) to afford the title compound (45) as a colorless oil.

Yield of 45 using 1 as vinylating reagent: $44.7 \mathrm{mg}, 68 \%$.

Yield of $\mathbf{4 5}$ using $\mathbf{3}$ as vinylating reagent: $9.3 \mathrm{mg}, 14 \%$. In addition, arylation product $\mathbf{4 6}$ was obtained as a colorless oil: $11.3 \mathrm{mg}, 14 \%$ yield.

\section{Data for 45:}

$\mathbf{R}_{\boldsymbol{f}}=0.30$ (EtOAc:pentane, 1:19 (v:v)).

\section{NMR Spectroscopy:}

${ }^{1} \mathrm{H}$ NMR $\left(300 \mathrm{MHz}, \mathrm{CDCl}_{3}, 298 \mathrm{~K}, \delta\right): 7.46(\mathrm{~s}, 1 \mathrm{H}), 7.27-7.17(\mathrm{~m}, 2 \mathrm{H}), 7.09$ (ddd, J=6.0, 3.1, $1.7 \mathrm{~Hz}$, $1 \mathrm{H}), 6.68$ (dd, $J=17.6,10.9 \mathrm{~Hz}, 1 \mathrm{H}), 6.53(\mathrm{~s}, 1 \mathrm{H}), 5.74$ (dd, $J=17.6,0.9 \mathrm{~Hz}, 1 \mathrm{H}), 5.24$ (dd, $J=10.9$, $0.9 \mathrm{~Hz}, 1 \mathrm{H}), 1.53(\mathrm{~s}, 9 \mathrm{H}) \mathrm{ppm}$. 
${ }^{13} \mathrm{C}$ NMR $\left(75 \mathrm{MHz}, \mathrm{CDCl}_{3}, 298 \mathrm{~K}, \delta\right): 152.9,138.8,138.6,136.8,129.2,121.1,118.1,116.4,114.4,80.7$, $28.5 \mathrm{ppm}$.

HRMS-El (m/z) calculated for $\mathrm{C}_{13} \mathrm{H}_{16} \mathrm{~N}_{1} \mathrm{O}_{2}+[\mathrm{M}]^{+}, 218.1188$; found, 218.1187; deviation: -0.8 ppm.

\section{Data for 46:}

$\mathbf{R}_{\boldsymbol{f}}=0.24$ (EtOAc:pentane, 1:19 (v:v)).

\section{NMR Spectroscopy:}

${ }^{1} \mathrm{H}$ NMR $\left(600 \mathrm{MHz}, \mathrm{CDCl}_{3}, 298 \mathrm{~K}, \delta\right): 7.63(\mathrm{~s}, 1 \mathrm{H}), 7.60-7.57(\mathrm{~m}, 2 \mathrm{H}), 7.45-7.40(\mathrm{~m}, 2 \mathrm{H}), 7.38-7.30$ (m, 3H), $7.28-7.25(\mathrm{~m}, 2 \mathrm{H}), 6.54(\mathrm{~s}, 1 \mathrm{H}), 1.54(\mathrm{~s}, 9 \mathrm{H}) \mathrm{ppm}$.

${ }^{13} \mathrm{C}$ NMR (151 MHz, $\left.\mathrm{CDCl}_{3}, 298 \mathrm{~K}, \delta\right):$ 153.0, 142.4, 141.1, 139.0, 129.6, 128.9, 127.6, 127.4, 122.2, 117.6, 117.5, 80.8, 28.6 ppm.

HRMS-El (m/z) calculated for $\mathrm{C}_{17} \mathrm{H}_{19} \mathrm{~N}_{1} \mathrm{O}_{2}{ }^{+}[\mathrm{M}]^{+}, 269.1409$; found, 269.1410; deviation: +0.3 ppm.

1-Fluoro-4-vinylbenzene (42)

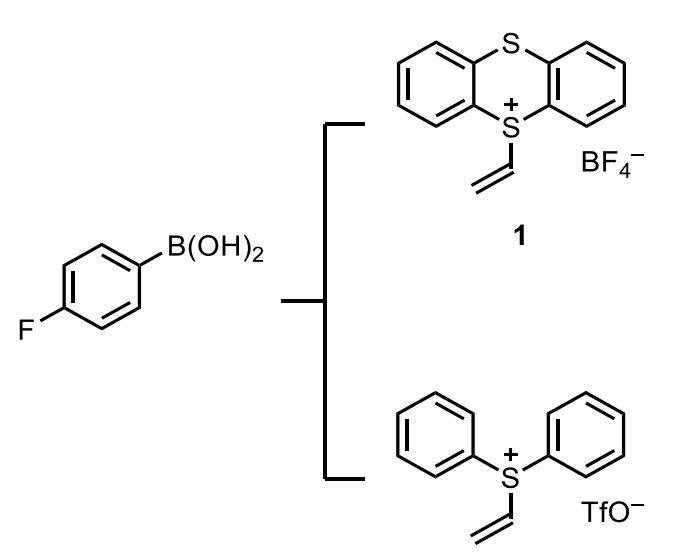

S3
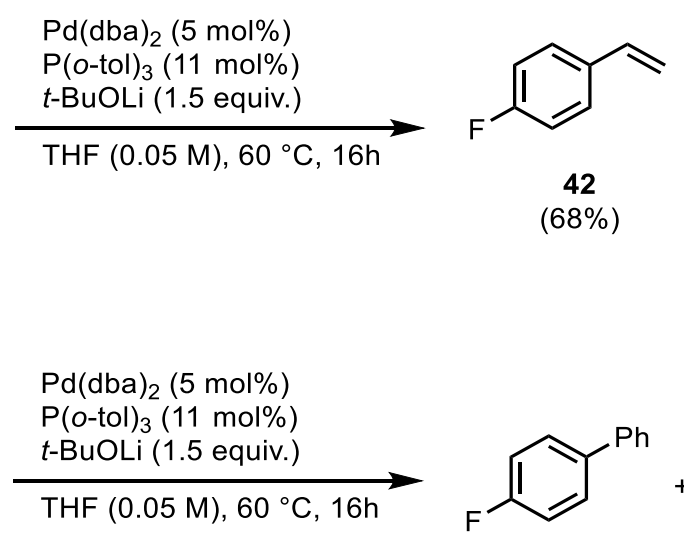

43

$(4 \%)$<smiles>C=Cc1ccc(F)cc1</smiles>

42

The title compound was prepared following general procedure B (for 1) or C (for S3). Under ambient atmosphere, a $4 \mathrm{~mL}$ vial equipped with a teflon-coated magnetic stirring bar was charged with (4fluorophenyl)boronic acid (7.0 mg, 0.050 mmol, 1.0 equiv.), Pd(dba) 2 (1.4 mg, 2.5 umol, 5.0 mol\%), P(o-tol) 3 (1.7 mg, $5.5 \mu \mathrm{mol}, 11 \mathrm{~mol} \%$ ) and $t$-BuOLi $(6.0 \mathrm{mg}, 0.075 \mathrm{mmol}, 1.5$ equiv.). The vial was transferred into a $\mathrm{N}_{2}$-filled glove box. Subsequently, dry THF $(1 \mathrm{~mL})$ was added into the vial. The reaction mixture was stirred for $2 \mathrm{~min}$ at $25^{\circ} \mathrm{C}$ before 1 ( $25 \mathrm{mg}, 0.075 \mathrm{mmol}, 1.5$ equiv.) was added into the vial in one portion. The vial was capped and was then transferred out of the glove box. The vial was placed on a heating block preheated at $60^{\circ} \mathrm{C}$ where the reaction mixture was stirred for $16 \mathrm{~h}$. The reaction mixture was cooled to $25^{\circ} \mathrm{C}$. To the cooled reaction mixture was added 4-fluorobenzotrifluoride (12.7 $\mu \mathrm{L}, 16.4 \mathrm{mg}, 0.10 \mathrm{mmol}, 2.0$ equiv.) as internal standard. The ${ }^{19} \mathrm{~F}$ NMR resonance of the product at $-114.6 \mathrm{ppm}$ was integrated relative to the ${ }^{19} \mathrm{~F}$ NMR resonances of the aromatic fluorine atom of 4 -fluorobenzotrifluoride $(\delta=-107.6 \mathrm{ppm})$. 
The scale of using $\mathbf{S 3}$ as vinylating reagent was doubled to $0.10 \mathrm{mmol}$. In this case, $0.10 \mathrm{mmol}$ (1.0 equiv.) of 4-fluorobenzotrifluoride was added as internal standard.

Yield of 42 using 1 as vinylating reagent: $68 \%$. Reaction run at $1.00 \mathrm{mmol}$ scale: $161 \mathrm{mg}, 74 \%$.

Yield of $\mathbf{4 2}$ using S3 as vinylating reagent: 4\%. In addition, arylation product $\mathbf{4 3}$ was obtained in $\mathbf{4 \%}$ yield.

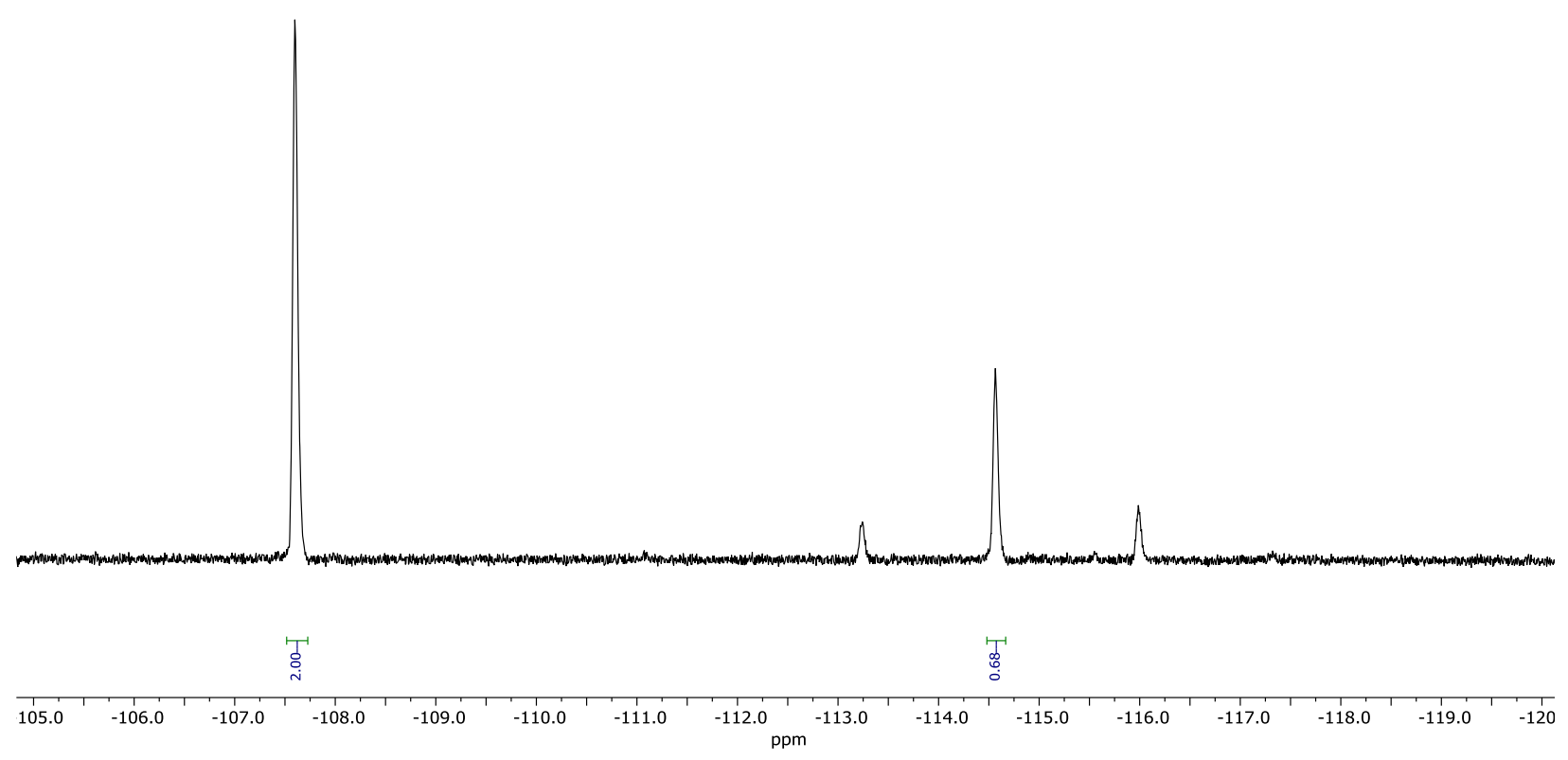

Figure S12. ${ }^{19} \mathrm{~F}$ NMR spectrum of the crude mixture of the Suzuki-type vinylation of (4-fluorophenyl)boronic acid with 1, THF, $471 \mathrm{MHz}, 298 \mathrm{~K}$. 


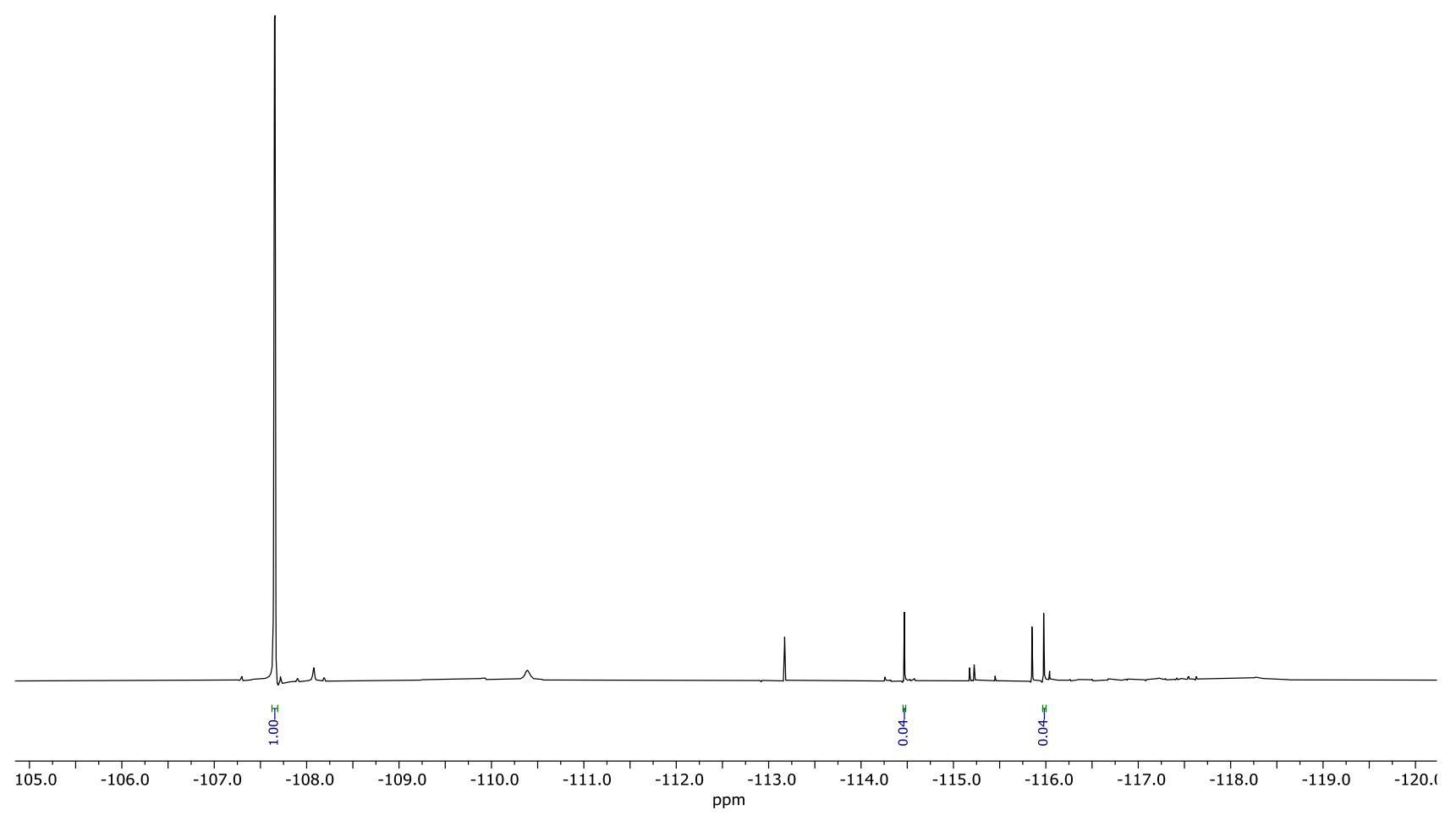

Figure S13. ${ }^{19} \mathrm{~F}$ NMR spectrum of the crude mixture of the Suzuki-type vinylation of (4-fluorophenyl)boronic acid with S3, THF, $282 \mathrm{MHz}, 298 \mathrm{~K}$.

\section{Discussion}

In stark difference to the direct nucleophilic substitution on thianthrenium salts, which often result in the endo $\mathrm{C}-\mathrm{S}$ bond cleavage and ring-opening of the thianthrene core, ${ }^{[6,12]}$ palladium-catalyzed reactions on these substrates generally result in the exo $\mathrm{C}-\mathrm{S}$ bond cleavage. ${ }^{[1,13-16]}$ This outcome may be rationalized considering that oxidative addition into the endocyclic $\mathrm{C}-\mathrm{S}$ bond may be reversible. ${ }^{[17-18]}$ This feature efficiently channels the reaction towards potentially irreversible cleavage of the exocyclic $\mathrm{C}($ vinyl)-S bond in reactions with 1 . In contrast, the reaction based on vinyl-SPh$(\mathrm{OTf})$ does not benefit from a similar behavior, which may the reason for the unselective $\mathrm{C}$ (vinyl)-S vs $\mathrm{C}$ (phenyl)-S bond cleavage under the same reaction conditions.

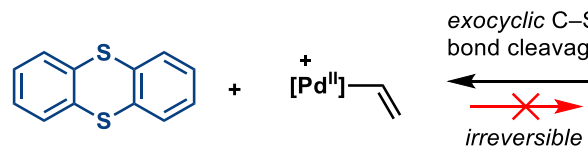

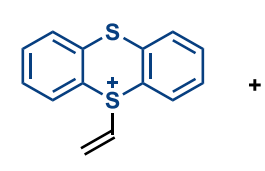

vs

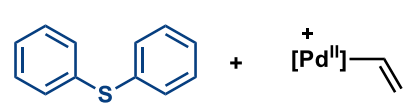

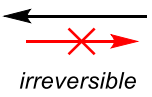<smiles>C=CS(=C1CCCCC1)c1ccccc1</smiles>

$\left[\mathrm{Pd}^{0}\right]$

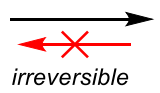

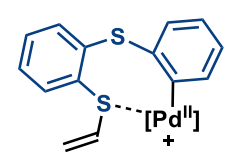<smiles>C=C[As]c1ccccc1</smiles>

In order to rule out the formation of $\mathrm{C}$-Ph products arising from an alternative pathway involving oxidative addition of $\mathrm{Ph}_{2} \mathrm{~S}$ into $\mathrm{Pd}(0)$ we set up the following experiments, described in the figure below: 
(1)

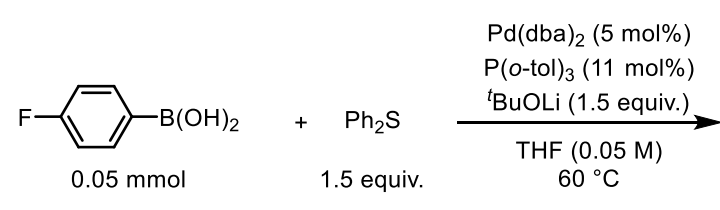

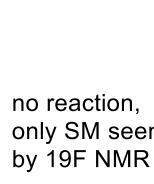

by 19 F NMR
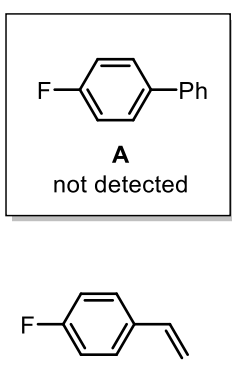

$60 \%$<smiles>Fc1ccccc1</smiles>

$35 \%$

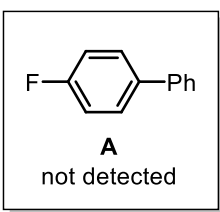

not detected

To assess the ability of $\mathrm{Ph}_{2} \mathrm{~S}$ to undergo oxidative addition to $\mathrm{Pd}(0)$ under our reaction conditions, we evaluated this possibility in reaction 1. Under the same conditions, the substitution of sulfonium salts by $\mathrm{Ph}_{2} \mathrm{~S}$ did not resulted in the formation of product $A$ and only boronic acid starting material was detected. Moreover, we run our reaction using vinyl-TT in presence of equimolar amounts of $\mathrm{Ph}_{2} \mathrm{~S}$ (reaction 2), but could not detect product A either. All observations are fully consistent with the original claims.

In addition, we also monitored the reaction profile of the reaction with vinyl-SPh$(\mathrm{OTf})$, which can be found in the following Figure:
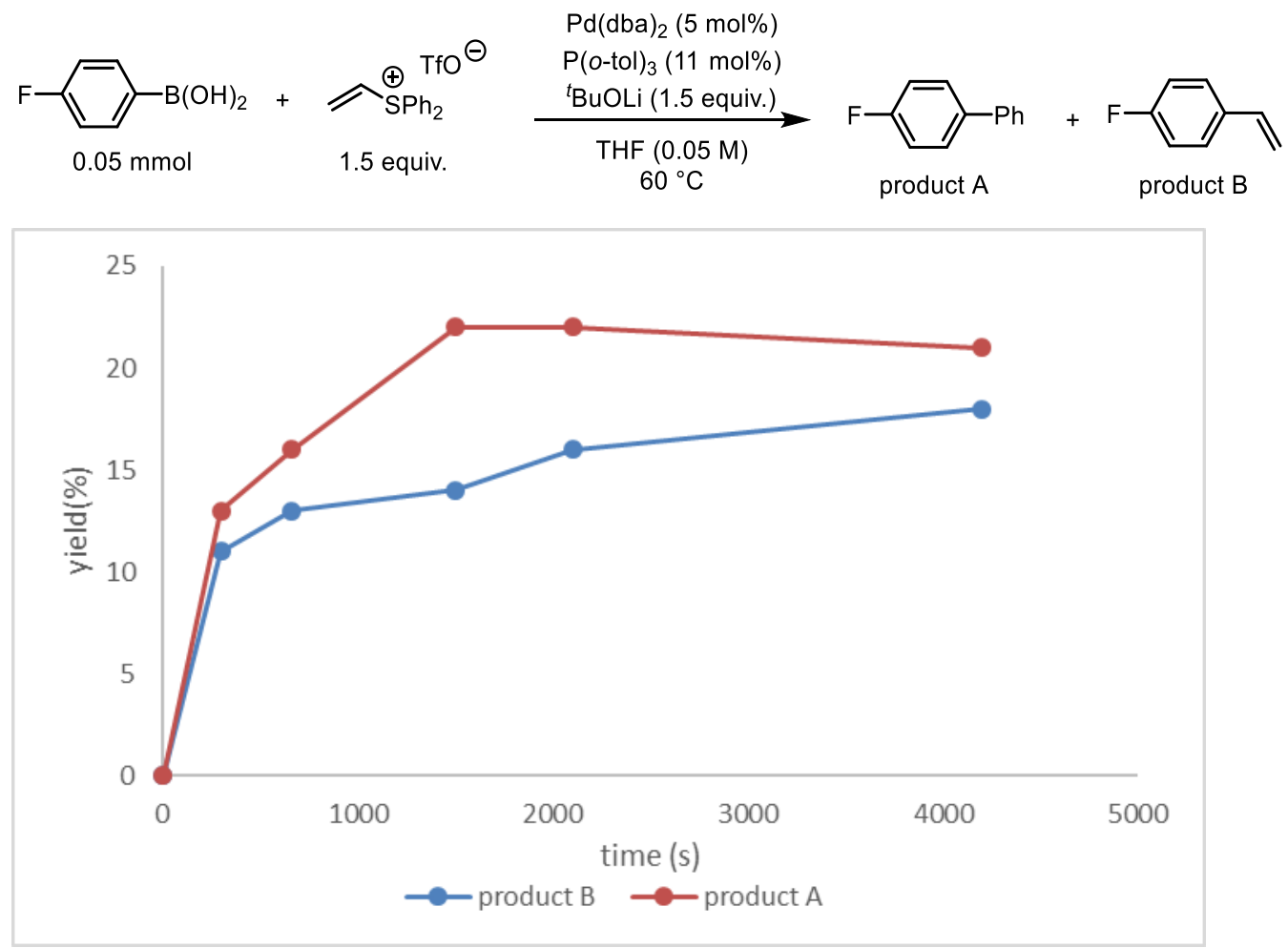

Our data shows that product $A$ is formed simultaneously to product $B$ since the beginning of the reaction, pointing against the formation of $\mathrm{Ph} 2 \mathrm{~S}$ and subsequent oxidative addition to afford product $\mathrm{A}$. 
Collectively, these data suggest that product A arises from Cphenyl-S oxidative addition on vinyl-SPh2(OTf) rather than oxidative addition on Ph2S.

\section{X-RAY CRYSTALLOGRAPHIC ANALYSIS}

\section{S-vinyl-thianthrenium tetrafluoroborate (vinyl-TT+, 1) (CCDC 2075820)}

\section{Experimental}

Vinyl- $\mathrm{TT}^{+}$(1) was crystallized from a dichloromethane/diethyl ether. The atoms are depicted with $50 \%$ probability ellipsoids. The crystallographic data are summarized in the following table.

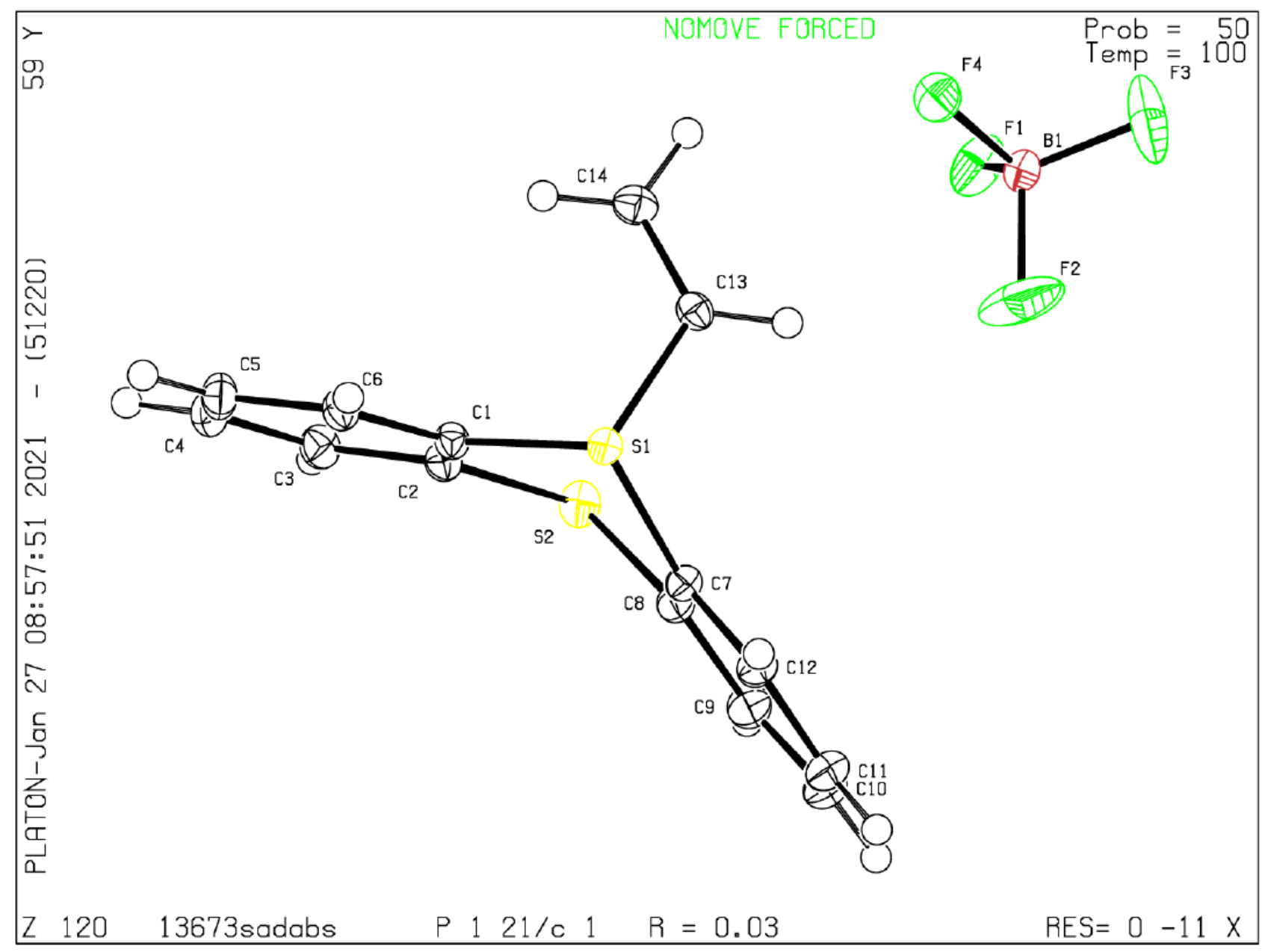

Figure S14. X-ray structure of vinyl-TT+ (1).

Table S4. Crystal data and structure refinement.

Empirical formula

Color

Formula weight
$\mathrm{C}_{14} \mathrm{H}_{11} \mathrm{BF}_{4} \mathrm{~S}_{2}$

colourless

$330.16 \mathrm{~g} \cdot \mathrm{mol}^{-1}$ 


\begin{tabular}{|c|c|c|}
\hline Temperature & \multicolumn{2}{|l|}{$100(2) \mathrm{K}$} \\
\hline Wavelength & \multicolumn{2}{|l|}{$0.71073 \AA$} \\
\hline Crystal system & \multicolumn{2}{|l|}{ MONOCLINIC } \\
\hline Space group & \multicolumn{2}{|l|}{$P 2_{1} / c$, (no. 14) } \\
\hline Unit cell dimensions & $\begin{array}{l}a=5.4988(4) \AA \\
b=18.2398(8) \AA \\
c=14.4633(12) \AA\end{array}$ & $\begin{array}{l}\alpha=90^{\circ} . \\
\beta=99.116(7)^{\circ} . \\
\gamma=90^{\circ} .\end{array}$ \\
\hline Volume & \multicolumn{2}{|l|}{$1432.30(17) \AA^{3}$} \\
\hline Z & \multicolumn{2}{|l|}{4} \\
\hline Density (calculated) & \multicolumn{2}{|l|}{$1.531 \mathrm{Mg} \cdot \mathrm{m}^{-3}$} \\
\hline Absorption coefficient & \multicolumn{2}{|l|}{$0.403 \mathrm{~mm}^{-1}$} \\
\hline$F(000)$ & \multicolumn{2}{|c|}{$672 \mathrm{e}$} \\
\hline Crystal size & \multicolumn{2}{|c|}{$0.18 \times 0.17 \times 0.13 \mathrm{~mm}^{3}$} \\
\hline$\theta$ range for data collection & \multicolumn{2}{|c|}{2.650 to $36.023^{\circ}$} \\
\hline Index ranges & \multicolumn{2}{|c|}{$-9 \leq \mathrm{h} \leq 9,-30 \leq \mathrm{k} \leq 30,-23 \leq \mathrm{I} \leq 23$} \\
\hline Reflections collected & \multicolumn{2}{|c|}{50539} \\
\hline Independent reflections & \multicolumn{2}{|l|}{$6779\left[R_{\text {int }}=0.0438\right]$} \\
\hline Reflections with $I>2 \sigma(I)$ & \multicolumn{2}{|l|}{5582} \\
\hline Completeness to $\theta=25.242^{\circ}$ & \multicolumn{2}{|l|}{$99.8 \%$} \\
\hline Absorption correction & \multicolumn{2}{|l|}{ Gaussian } \\
\hline Max. and min. transmission & \multicolumn{2}{|c|}{0.96 and 0.91} \\
\hline Refinement method & \multicolumn{2}{|c|}{ Full-matrix least-squares on $\mathrm{F}^{2}$} \\
\hline Data / restraints / parameters & \multicolumn{2}{|c|}{$6779 / 0$ / 202} \\
\hline Goodness-of-fit on $F^{2}$ & \multicolumn{2}{|l|}{1.040} \\
\hline Final $R$ indices $[\mid>2 \square(I)]$ & $\mathrm{R}_{1}=0.0324$ & $w R^{2}=0.0793$ \\
\hline $\mathrm{R}$ indices (all data) & $\mathrm{R}_{1}=0.0441$ & $w R^{2}=0.0845$ \\
\hline Largest diff. peak and hole & 1.0 and $-0.5 \mathrm{e} \cdot \AA^{-3}$ & \\
\hline
\end{tabular}

Table S5. Bond lengths $[\AA ̊]$ and angles $\left[^{\circ}\right]$

\begin{tabular}{llll}
\hline $\mathrm{S}(1)-\mathrm{C}(1)$ & $1.7635(9)$ & $\mathrm{S}(1)-\mathrm{C}(7)$ & $1.7718(9)$ \\
$\mathrm{S}(1)-\mathrm{C}(13)$ & $1.7857(9)$ & $\mathrm{S}(2)-\mathrm{C}(2)$ & $1.7613(9)$ \\
$\mathrm{S}(2)-\mathrm{C}(8)$ & $1.7634(10)$ & $\mathrm{C}(1)-\mathrm{C}(2)$ & $1.3996(12)$ \\
$\mathrm{C}(1)-\mathrm{C}(6)$ & $\mathrm{C}(2)-\mathrm{C}(3)$ & $1.3989(13)$ \\
$\mathrm{C}(3)-\mathrm{C}(4)$ & $1.3950(12)$ & $\mathrm{C}(4)-\mathrm{C}(5)$ & $1.3905(14)$ \\
$\mathrm{C}(5)-\mathrm{C}(6)$ & $1.3909(14)$ & $\mathrm{C}(7)-\mathrm{C}(8)$ & $1.3972(12)$ \\
$\mathrm{C}(7)-\mathrm{C}(12)$ & $1.3890(13)$ & $\mathrm{C}(8)-\mathrm{C}(9)$ & $1.4012(13)$ \\
$\mathrm{C}(9)-\mathrm{C}(10)$ & $1.3965(13)$ & $\mathrm{C}(10)-\mathrm{C}(11)$ & $1.3929(17)$ \\
$\mathrm{C}(11)-\mathrm{C}(12)$ & $\mathrm{C}(13)-\mathrm{H}(13)$ & $0.945(16)$ \\
$\mathrm{C}(13)-\mathrm{C}(14)$ & $1.3890(16)$ & $\mathrm{C}(14)-\mathrm{H}(14 \mathrm{~A})$ & $0.961(16)$ \\
$\mathrm{C}(14)-\mathrm{H}(14 \mathrm{~B})$ & $1.3907(14)$ & $\mathrm{F}(1)-\mathrm{B}(1)$ & $1.3924(12)$ \\
$\mathrm{F}(2)-\mathrm{B}(1)$ & $1.3176(13)$ & $\mathrm{F}(3)-\mathrm{B}(1)$ & \\
$\mathrm{F}(4)-\mathrm{B}(1)$ & $0.941(15)$ & & \\
& $1.3804(13)$ & & $1.3766(13)$ \\
$\mathrm{C}(1)-\mathrm{S}(1)-\mathrm{C}(7)$ & $1.3920(12)$ & $\mathrm{C}(1)-\mathrm{S}(1)-\mathrm{C}(13)$ & \\
$\mathrm{C}(7)-\mathrm{S}(1)-\mathrm{C}(13)$ & & $\mathrm{C}(2)-\mathrm{S}(2)-\mathrm{C}(8)$ & $103.57(4)$ \\
$\mathrm{C}(2)-\mathrm{C}(1)-\mathrm{S}(1)$ & & $\mathrm{C}(6)-\mathrm{C}(1)-\mathrm{S}(1)$ & $116.80(4)$ \\
$\mathrm{C}(6)-\mathrm{C}(1)-\mathrm{C}(2)$ & $102.27(4)$ & $\mathrm{C}(1)-\mathrm{C}(2)-\mathrm{S}(2)$ & $123.47(7)$ \\
$\mathrm{C}(3)-\mathrm{C}(2)-\mathrm{S}(2)$ & $101.29(4)$ & $\mathrm{C}(3)-\mathrm{C}(2)-\mathrm{C}(1)$ & $118.39(8)$
\end{tabular}


$\mathrm{C}(4)-\mathrm{C}(3)-\mathrm{C}(2)$

$\mathrm{C}(6)-\mathrm{C}(5)-\mathrm{C}(4)$

$\mathrm{C}(8)-\mathrm{C}(7)-\mathrm{S}(1)$

$\mathrm{C}(12)-\mathrm{C}(7)-\mathrm{C}(8)$

$\mathrm{C}(7)-\mathrm{C}(8)-\mathrm{C}(9)$

$\mathrm{C}(10)-\mathrm{C}(9)-\mathrm{C}(8)$

C(12)-C(11)-C(10)

$\mathrm{S}(1)-\mathrm{C}(13)-\mathrm{H}(13)$

$\mathrm{C}(14)-\mathrm{C}(13)-\mathrm{H}(13)$

$\mathrm{C}(13)-\mathrm{C}(14)-\mathrm{H}(14 \mathrm{~B})$

$F(2)-B(1)-F(1)$

$F(3)-B(1)-F(1)$

$F(3)-B(1)-F(4)$
120.04(8)

120.12(9)

120.94(7)

121.82(8)

$118.27(9)$

$120.19(10)$

$120.00(10)$

$112.2(10)$

125.6(10)

123.4(9)

108.18(9)

109.33(9)

109.94(8)
$\mathrm{C}(5)-\mathrm{C}(4)-\mathrm{C}(3)$

$\mathrm{C}(5)-\mathrm{C}(6)-\mathrm{C}(1)$

$\mathrm{C}(12)-\mathrm{C}(7)-\mathrm{S}(1)$

$\mathrm{C}(7)-\mathrm{C}(8)-\mathrm{S}(2)$

$\mathrm{C}(9)-\mathrm{C}(8)-\mathrm{S}(2)$

$\mathrm{C}(9)-\mathrm{C}(10)-\mathrm{C}(11)$

C(11)-C(12)-C(7)

$C(14)-C(13)-S(1)$

$\mathrm{C}(13)-\mathrm{C}(14)-\mathrm{H}(14 \mathrm{~A})$

$\mathrm{H}(14 \mathrm{~A})-\mathrm{C}(14)-\mathrm{H}(14 \mathrm{~B})$

$F(2)-B(1)-F(4)$

$F(3)-B(1)-F(2)$

$F(4)-B(1)-F(1)$
120.78(9)

$118.90(8)$

$117.21(7)$

$123.80(7)$

117.92(7)

120.74(9)

$118.89(9)$

$122.05(7)$

119.6(9)

117.0(13)

109.52(9)

$110.56(10)$

109.28(8)

\section{[4+2] cycloadduct intermediate 3 (CCDC 2075821)}

\section{Experimental}

[4+2] cycloadduct intermediate 3 was crystallized from acetone/diethyl ether. The atoms are depicted with $50 \%$ probability ellipsoids. The crystallographic data are summarized in the following table.

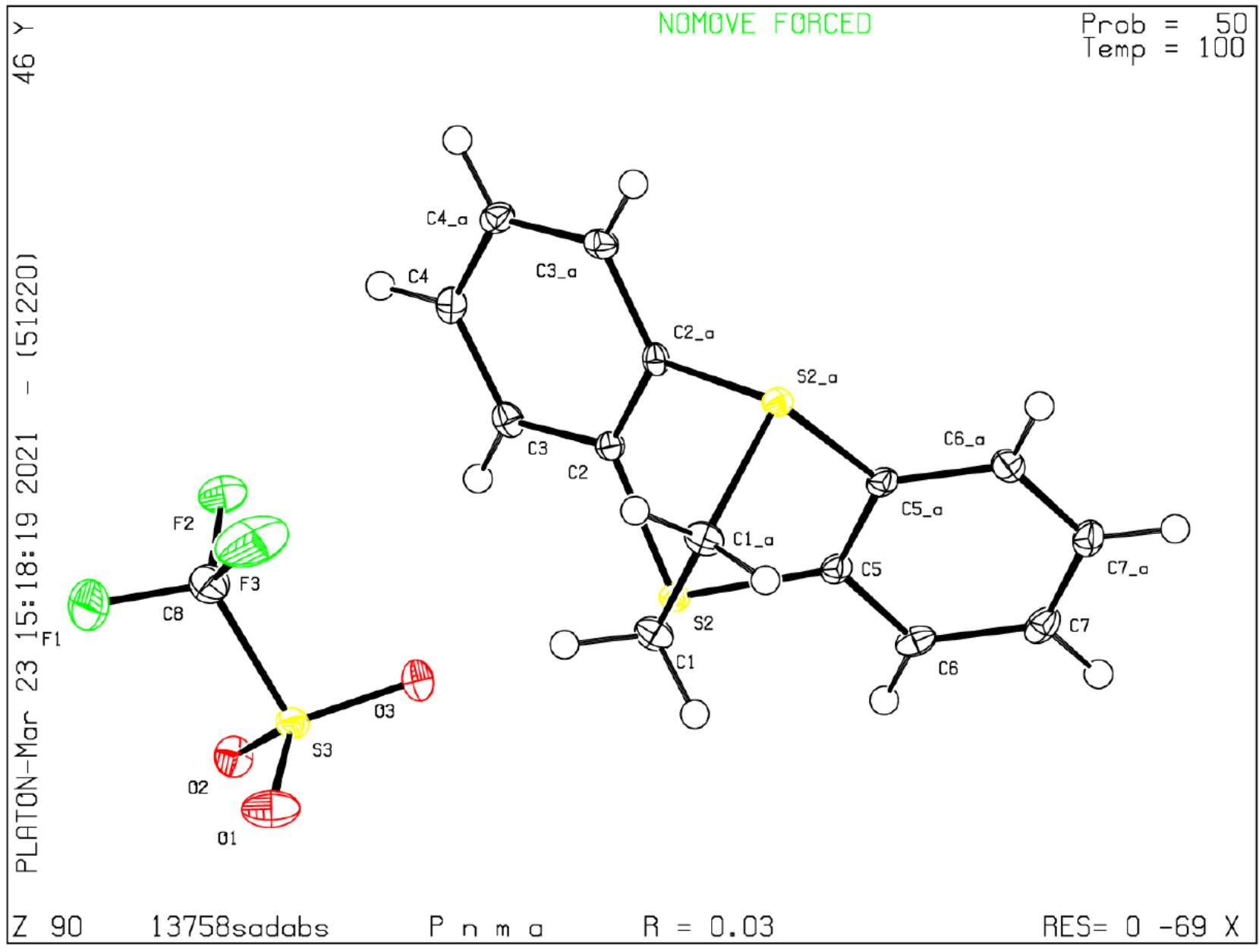

Figure S15. X-ray structure of [4+2] cycloadduct intermediate 3. 
Table S6. Crystal data and structure refinement.

\begin{tabular}{|c|c|c|}
\hline Empirical formula & \multicolumn{2}{|l|}{$\mathrm{C}_{16} \mathrm{H}_{12} \mathrm{~F}_{6} \mathrm{O}_{6} \mathrm{~S}_{4}$} \\
\hline Color & \multicolumn{2}{|l|}{ colourless } \\
\hline Formula weight & \multicolumn{2}{|l|}{$542.50 \mathrm{~g} \cdot \mathrm{mol}^{-1}$} \\
\hline Temperature & \multicolumn{2}{|l|}{$100(2) \mathrm{K}$} \\
\hline Wavelength & \multicolumn{2}{|l|}{$1.54178 \AA$} \\
\hline Crystal system & \multicolumn{2}{|l|}{ ORTHORHOMBIC } \\
\hline Space group & \multicolumn{2}{|l|}{ Pnma, (no. 62) } \\
\hline \multirow[t]{3}{*}{ Unit cell dimensions } & $a=6.6681(2) \AA$ & $\alpha=90^{\circ}$. \\
\hline & $b=14.5847(5) \AA$ & $\beta=90^{\circ}$. \\
\hline & $c=21.1524(7) \AA$ & $\gamma=90^{\circ}$ \\
\hline Volume & \multicolumn{2}{|l|}{ 2057.12(12) $\AA^{3}$} \\
\hline Z & \multicolumn{2}{|l|}{4} \\
\hline Density (calculated) & \multicolumn{2}{|l|}{$1.752 \mathrm{Mg} \cdot \mathrm{m}^{-3}$} \\
\hline Absorption coefficient & \multicolumn{2}{|l|}{$5.083 \mathrm{~mm}^{-1}$} \\
\hline$F(000)$ & \multicolumn{2}{|c|}{$1096 \mathrm{e}$} \\
\hline Crystal size & \multicolumn{2}{|c|}{$0.261 \times 0.090 \times 0.040 \mathrm{~mm}^{3}$} \\
\hline$\theta$ range for data collection & \multicolumn{2}{|c|}{3.681 to $72.123^{\circ}$} \\
\hline Index ranges & \multicolumn{2}{|c|}{$-7 \leq \mathrm{h} \leq 8,-17 \leq \mathrm{k} \leq 17,-26 \leq \mathrm{I} \leq 26$} \\
\hline Reflections collected & \multicolumn{2}{|c|}{61680} \\
\hline Independent reflections & \multicolumn{2}{|l|}{$2096\left[R_{\text {int }}=0.0788\right]$} \\
\hline Reflections with $\mid>2 \sigma(I)$ & \multicolumn{2}{|l|}{1790} \\
\hline Completeness to $\theta=67.679^{\circ}$ & \multicolumn{2}{|l|}{$99.9 \%$} \\
\hline Absorption correction & \multicolumn{2}{|l|}{ Gaussian } \\
\hline Max. and min. transmission & \multicolumn{2}{|l|}{0.89 and 0.63} \\
\hline Refinement method & \multicolumn{2}{|c|}{ Full-matrix least-squares on $\mathrm{F}^{2}$} \\
\hline Data / restraints / parameters & \multicolumn{2}{|c|}{$2096 / 0 / 145$} \\
\hline Goodness-of-fit on $F^{2}$ & \multicolumn{2}{|l|}{1.159} \\
\hline Final $R$ indices $[I>2 \sigma(I)]$ & $\mathrm{R}_{1}=0.0331$ & $w R^{2}=0.0915$ \\
\hline $\mathrm{R}$ indices (all data) & $\mathrm{R}_{1}=0.0447$ & $w R^{2}=0.0999$ \\
\hline Largest diff. peak and hole & 0.5 and $-0.6 \mathrm{e} \cdot \AA^{-3}$ & \\
\hline
\end{tabular}

Table S7. Bond lengths $[\AA]$ and angles $\left[{ }^{\circ}\right]$

\begin{tabular}{llll}
\hline $\mathrm{S}(1)-\mathrm{C}(1)$ & $1.828(2)$ & $\mathrm{S}(1)-\mathrm{C}(2)$ & $1.7762(19)$ \\
$\mathrm{S}(1)-\mathrm{C}(5)$ & $1.7766(19)$ & $\mathrm{C}(1)-\mathrm{C}(1)^{*}$ & $1.526(4)$ \\
$\mathrm{C}(2)-\mathrm{C}(2)^{*}$ & $1.391(4)$ & $\mathrm{C}(2)-\mathrm{C}(3)$ & $1.387(3)$ \\
$\mathrm{C}(3)-\mathrm{C}(4)$ & $1.391(3)$ & $\mathrm{C}(4)-\mathrm{C}(4)^{*}$ & $1.398(4)$ \\
$\mathrm{C}(5)-\mathrm{C}(5)^{\star}$ & $1.392(4)$ & $\mathrm{C}(5)-\mathrm{C}(6)$ & $1.384(3)$ \\
$\mathrm{C}(6)-\mathrm{C}(7)$ & $1.391(3)$ & $\mathrm{C}(7)-\mathrm{C}(7)^{*}$ & $1.390(4)$ \\
$\mathrm{S}(2)-\mathrm{O}(1)$ & $1.4367(17)$ & $\mathrm{S}(2)-\mathrm{O}(2)$ & $1.4478(14)$ \\
$\mathrm{S}(2)-\mathrm{O}(3)$ & $1.4385(16)$ & $\mathrm{S}(2)-\mathrm{C}(8)$ & $1.822(2)$ \\
$\mathrm{F}(1)-\mathrm{C}(8)$ & $1.329(3)$ & $\mathrm{F}(2)-\mathrm{C}(8)$ & $1.334(3)$ \\
$\mathrm{F}(3)-\mathrm{C}(8)$ & $1.326(3)$ & &
\end{tabular}


$\mathrm{C}(2)-S(1)-C(1)$

$\mathrm{C}(5)-S(1)-C(1)$

$\mathrm{C}(2)^{*}-\mathrm{C}(2)-\mathrm{S}(1)$

$\mathrm{C}(3)-\mathrm{C}(2)-\mathrm{C}(2)$

$\mathrm{C}(3)-\mathrm{C}(4)-\mathrm{C}(4)^{*}$

$\mathrm{C}(6)-\mathrm{C}(5)-\mathrm{S}(1)$

$\mathrm{C}(5)-\mathrm{C}(6)-\mathrm{C}(7)$

$\mathrm{O}(1)-\mathrm{S}(2)-\mathrm{O}(2)$

$\mathrm{O}(1)-\mathrm{S}(2)-\mathrm{C}(8)$

$\mathrm{O}(3)-\mathrm{S}(2)-\mathrm{O}(2)$

$\mathrm{F}(1)-\mathrm{C}(8)-\mathrm{S}(2)$

$F(2)-C(8)-S(2)$

$\mathrm{F}(3)-\mathrm{C}(8)-\mathrm{F}(1)$
97.93(9)

97.03(9)

$119.32(6)$

121.02(12)

120.75(12)

119.51(16)

$118.3(2)$

114.06(9)

104.17(10)

$114.09(10)$

111.68(14)

$110.82(15)$

108.36(19)
C(2)-S(1)-C(5)

$C(1)^{*}-C(1)-S(1)$

$\mathrm{C}(3)-\mathrm{C}(2)-\mathrm{S}(1)$

$\mathrm{C}(2)-\mathrm{C}(3)-\mathrm{C}(4)$

$\mathrm{C}(5)^{*}-\mathrm{C}(5)-\mathrm{S}(1)$

$\mathrm{C}(6)-\mathrm{C}(5)-\mathrm{C}(5)^{*}$

$\mathrm{C}(7)^{*}-\mathrm{C}(7)-\mathrm{C}(6)$

$\mathrm{O}(1)-\mathrm{S}(2)-\mathrm{O}(3)$

$\mathrm{O}(2)-\mathrm{S}(2)-\mathrm{C}(8)$

$\mathrm{O}(3)-\mathrm{S}(2)-\mathrm{C}(8)$

$F(1)-C(8)-F(2)$

$\mathrm{F}(3)-\mathrm{C}(8)-\mathrm{S}(2)$

$F(3)-C(8)-F(2)$
103.06(9)

116.03(6)

119.41(15)

118.22(19)

119.29(7)

120.93(12)

120.82(12)

115.79(10)

103.44(9)

103.19(10)

107.09(17)

110.99(15)

107.73(18)

Symmetry transformations used to generate equivalent atoms: ${ }^{*} \mathrm{x},-\mathrm{y}+3 / 2, \mathrm{z}$ 
SPECTROSCOPIC DATA

\section{${ }^{1} \mathrm{H}$ NMR of vinyl TT+ (1)}

$\mathrm{CD}_{3} \mathrm{CN}, 298 \mathrm{~K}$

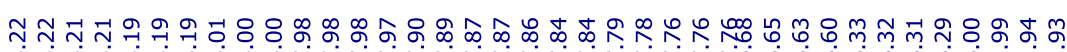

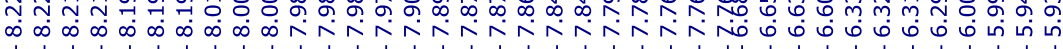

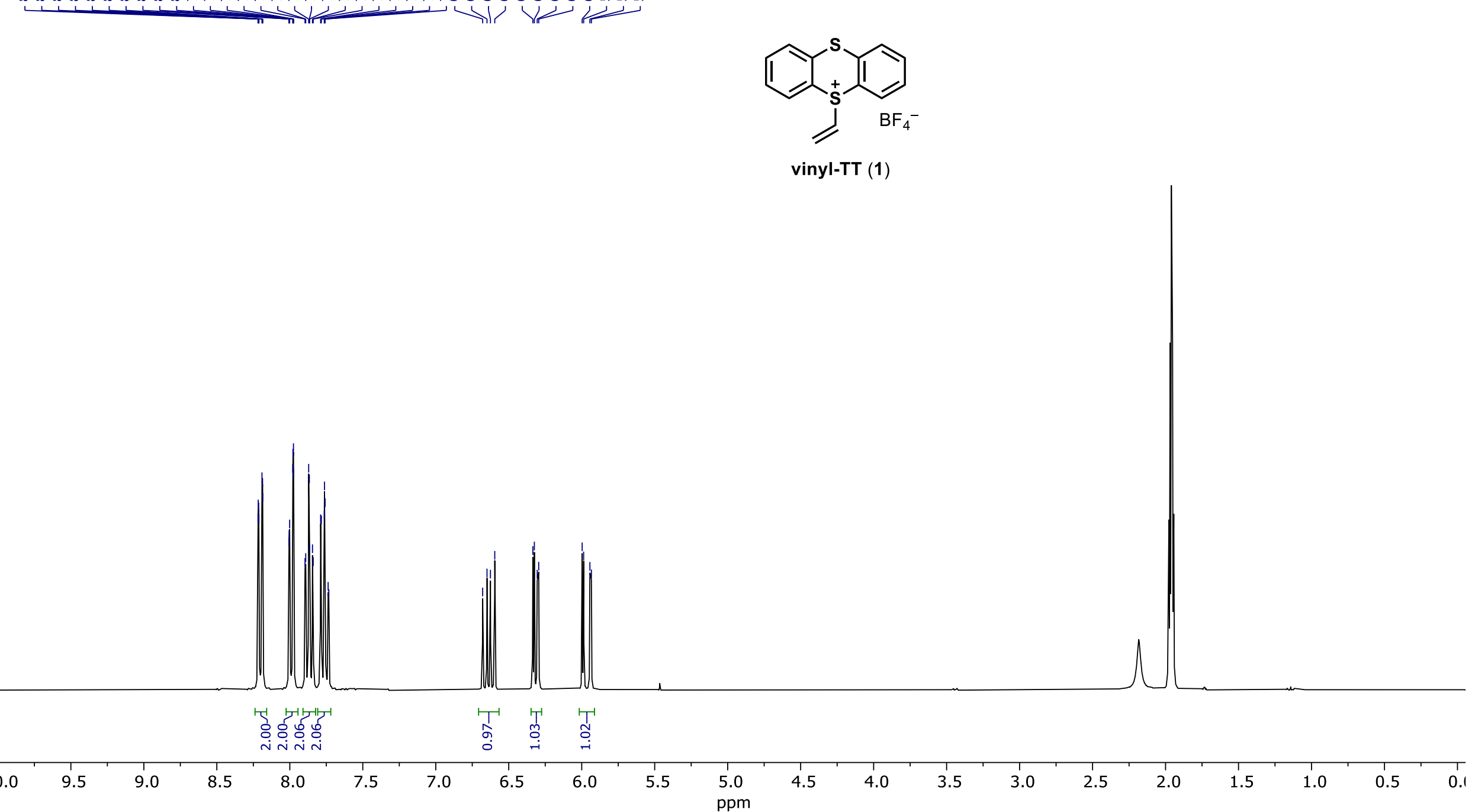


${ }^{13} \mathrm{C}$ NMR of vinyl TT+ (1)

$\mathrm{CD}_{3} \mathrm{CN}, 298 \mathrm{~K}$
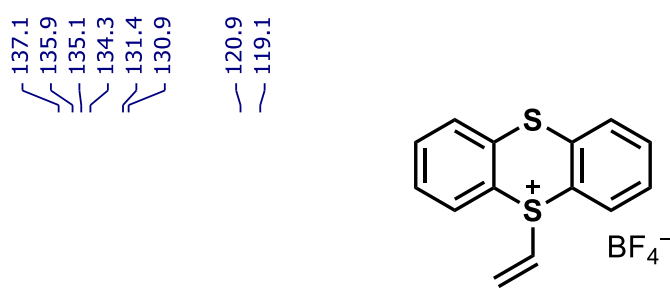

vinyl-TT (1)

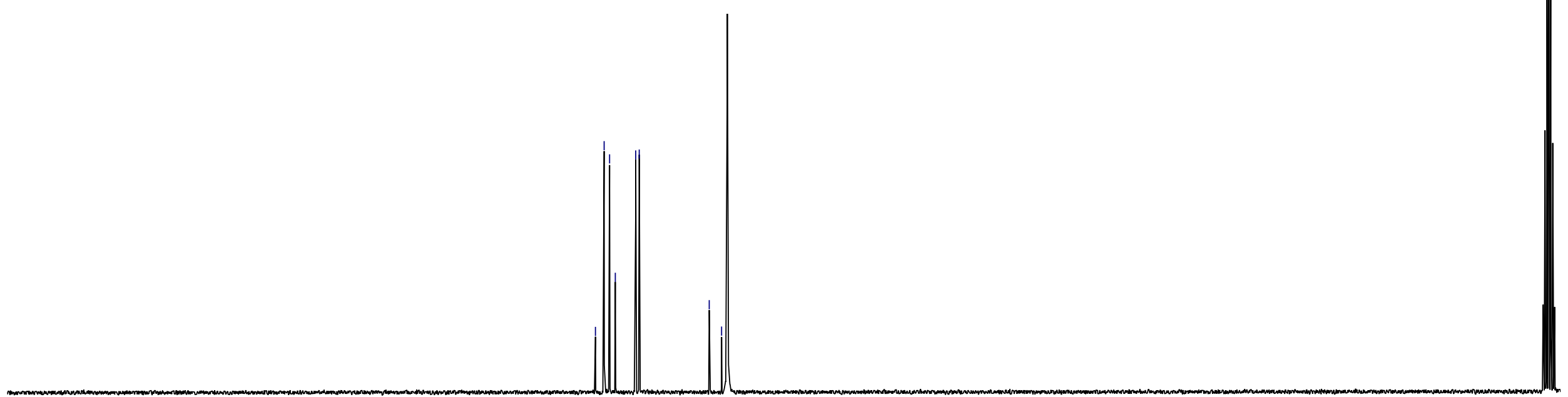

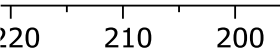


${ }^{19} \mathrm{~F}$ NMR of vinyl TT+ (1)

$\mathrm{CD}_{3} \mathrm{CN}, 298 \mathrm{~K}$

i

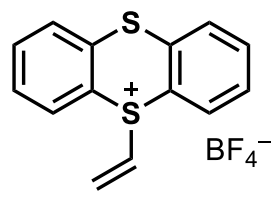

vinyl-TT (1)

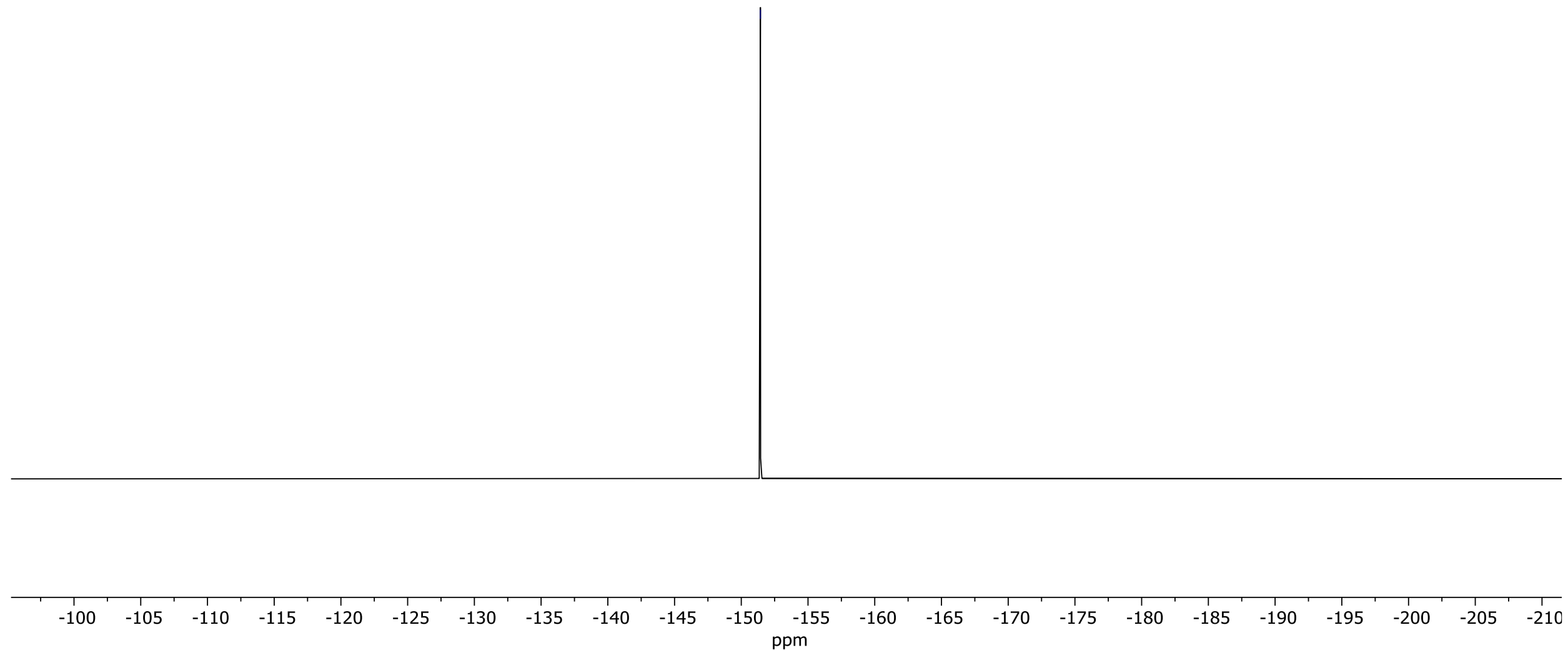




\section{${ }^{1} \mathrm{H}$ NMR of vinyl TT ${ }^{+}-d_{3}\left(1-d_{3}\right)$}

$\mathrm{CD}_{2} \mathrm{Cl}_{2}, 298 \mathrm{~K}$

$\underbrace{\underbrace{\infty}_{\infty}}$

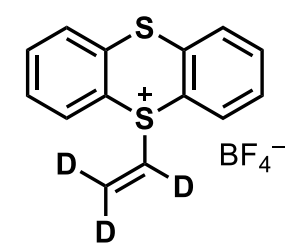

vinyl-TT+ $-d_{3}\left(1-d_{3}\right)$

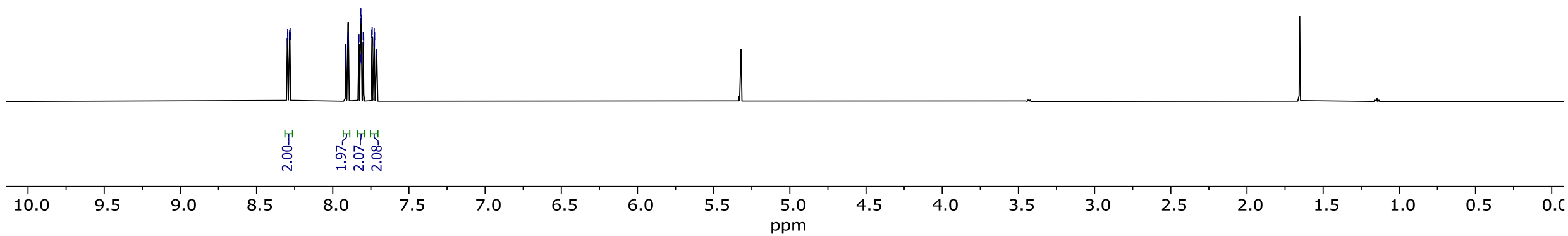




\section{${ }^{2} \mathrm{H}$ NMR of vinyl TT+- $d_{3}\left(1-d_{3}\right)$}

$\mathrm{CD}_{2} \mathrm{Cl}_{2}, 298 \mathrm{~K}$

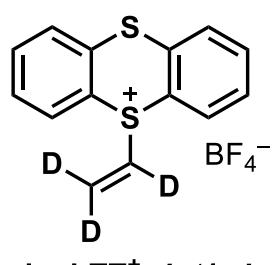

vinyl-TT+ $-d_{3}\left(1-d_{3}\right)$

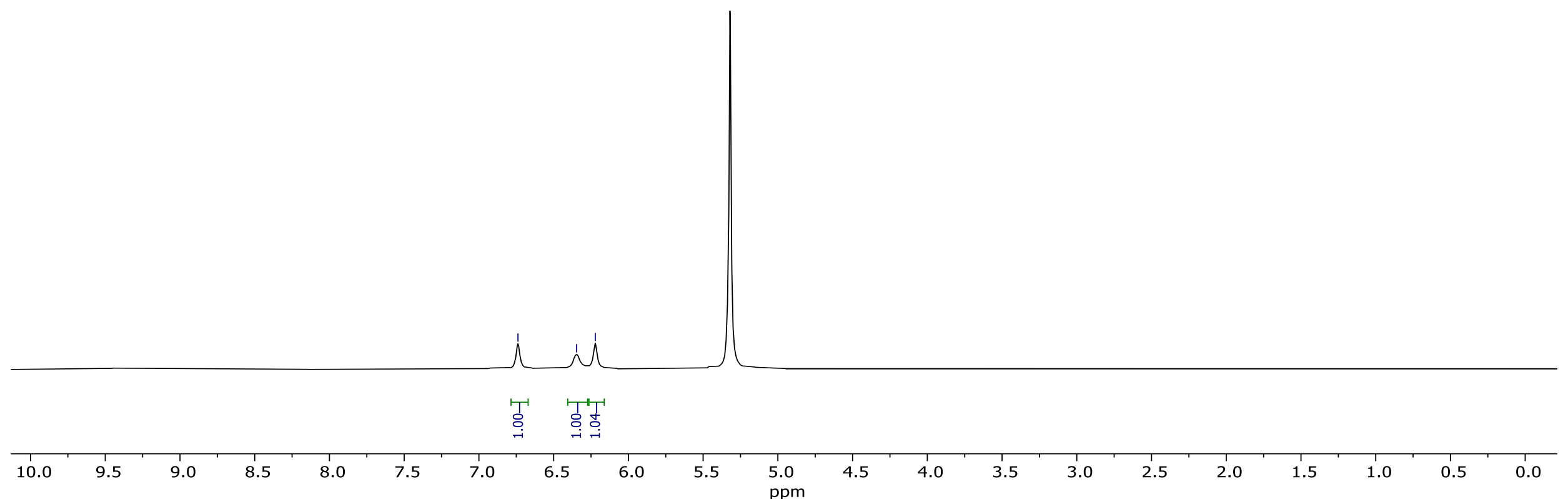




\section{${ }^{13} \mathrm{C}$ NMR of vinyl TT ${ }^{+}-d_{3}\left(1-d_{3}\right)$}

$\mathrm{CD}_{2} \mathrm{Cl}_{2}, 298 \mathrm{~K}$

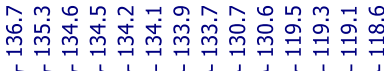 \\ 然}

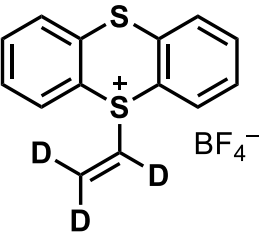

vinyl- $\mathrm{TT}^{+}-d_{3}\left(1-d_{3}\right)$

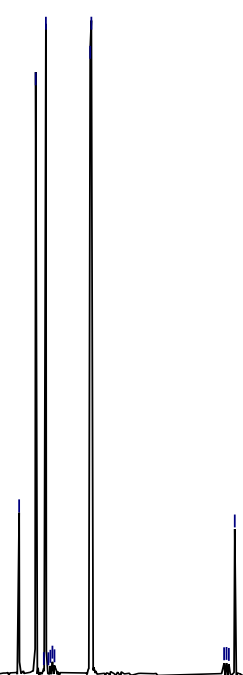


${ }^{19} \mathrm{~F}$ NMR of vinyl TT ${ }^{+}-d_{3}\left(1-d_{3}\right)$

$\mathrm{CD}_{2} \mathrm{Cl}_{2}, 298 \mathrm{~K}$

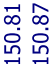

i

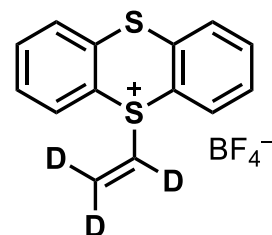

vinyl- $\mathrm{TT}^{+}-d_{3}\left(1-d_{3}\right.$

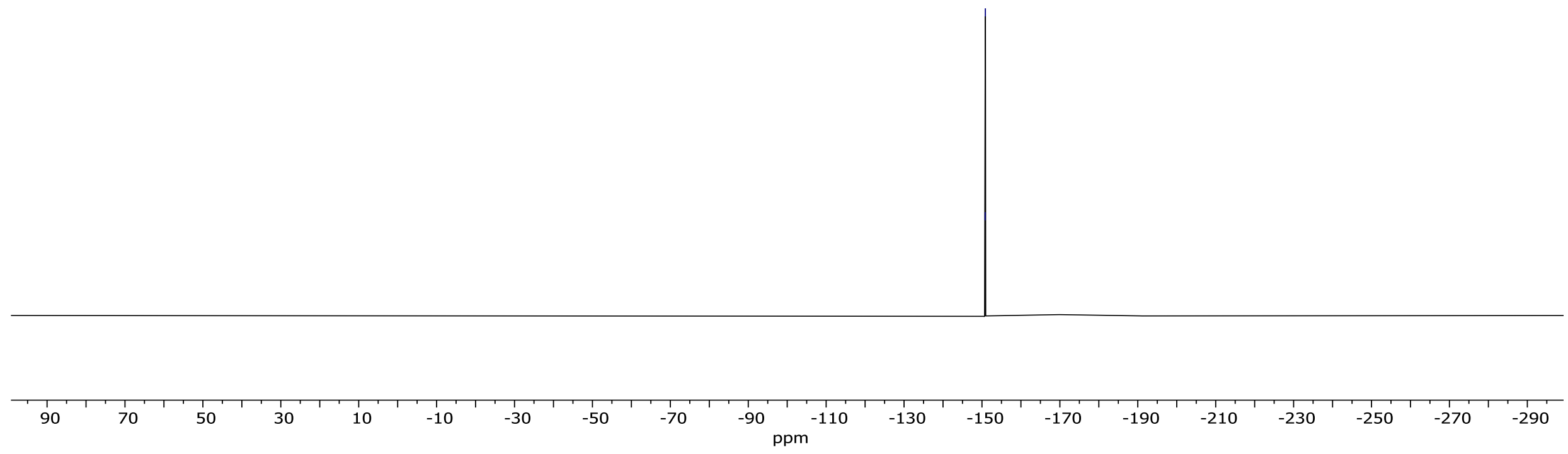


${ }^{1} \mathrm{H}$ NMR of the [4+2] cycloadduct intermediate 3

$\mathrm{CD}_{3} \mathrm{CN}, 23 \stackrel{\circ}{\circ} \mathrm{C}$

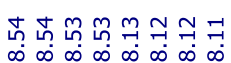

$\underbrace{\infty}$

$\stackrel{\infty}{\stackrel{\infty}{+}}$
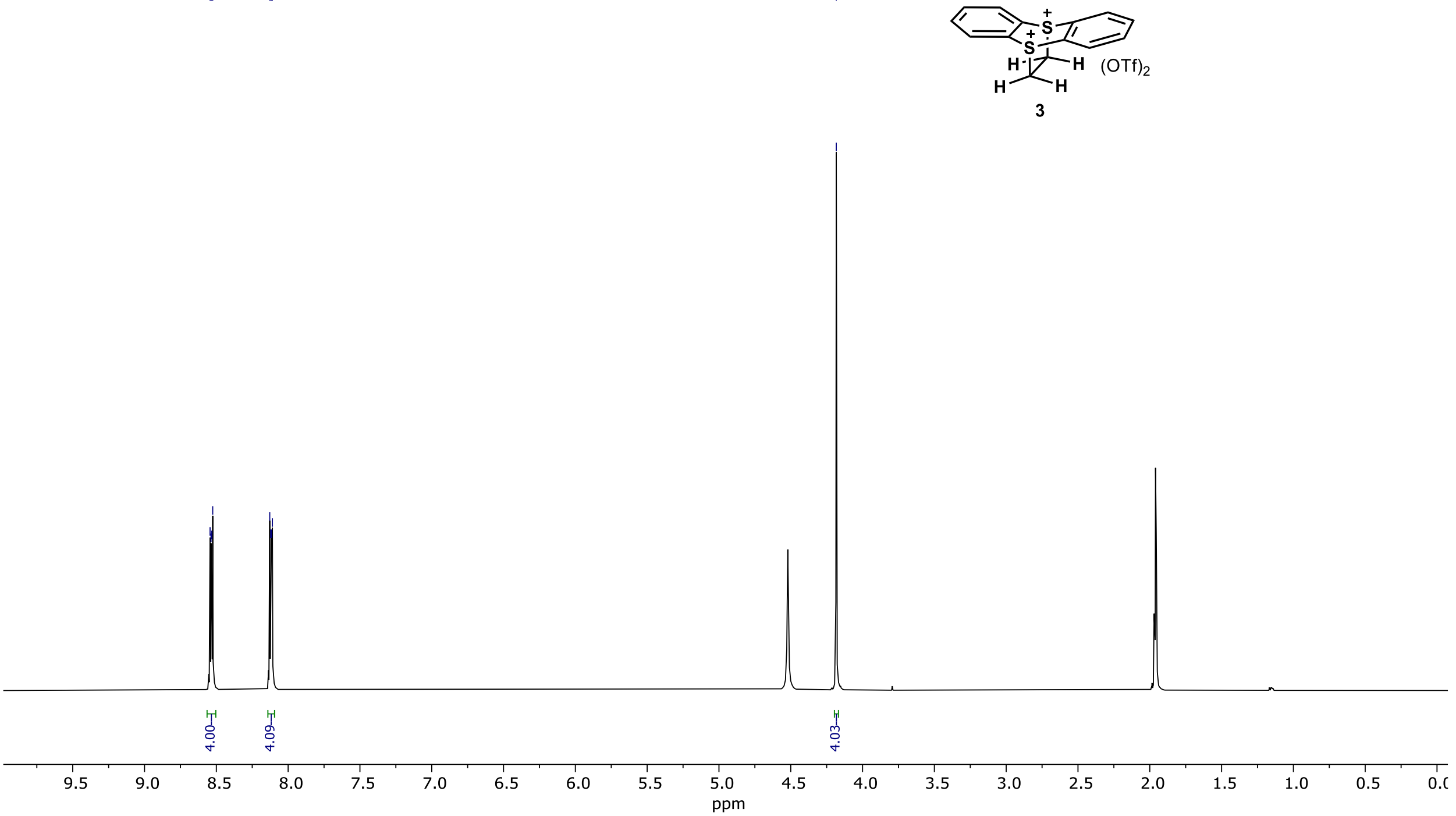
${ }^{13} \mathrm{C}$ NMR of the [4+2] cycloadduct intermediate 3

$\mathrm{CD}_{3} \mathrm{CN}, 23 \stackrel{\circ}{\mathrm{C}}$

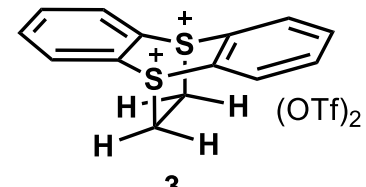

3

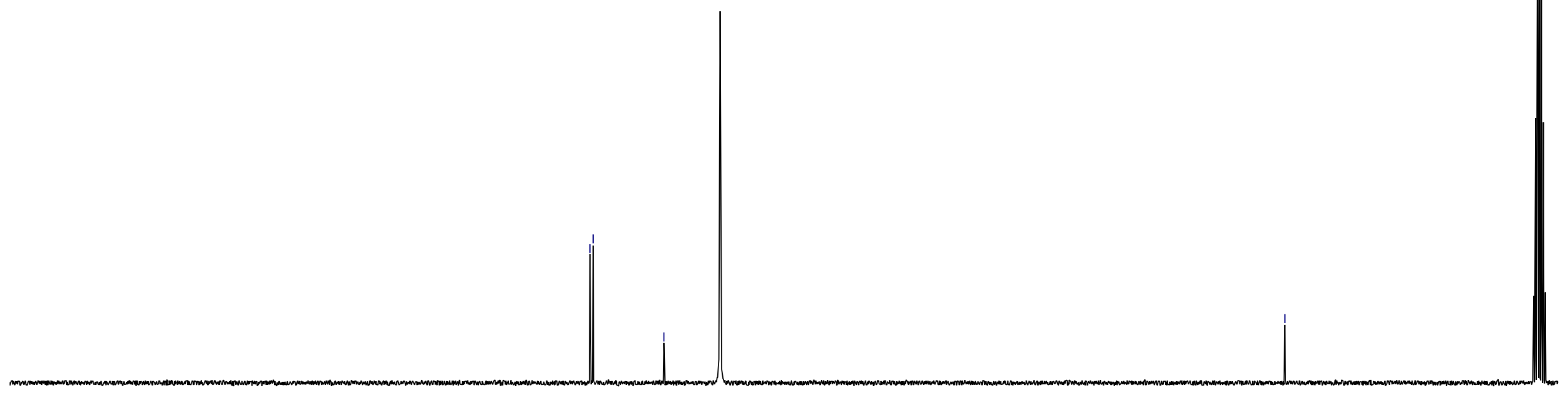

$210 \quad 200$ 
${ }^{19} \mathrm{~F}$ NMR of the [4+2] cycloadduct intermediate 3

$\mathrm{CD}_{3} \mathrm{CN}, 23 \stackrel{\circ}{\mathrm{C}}$
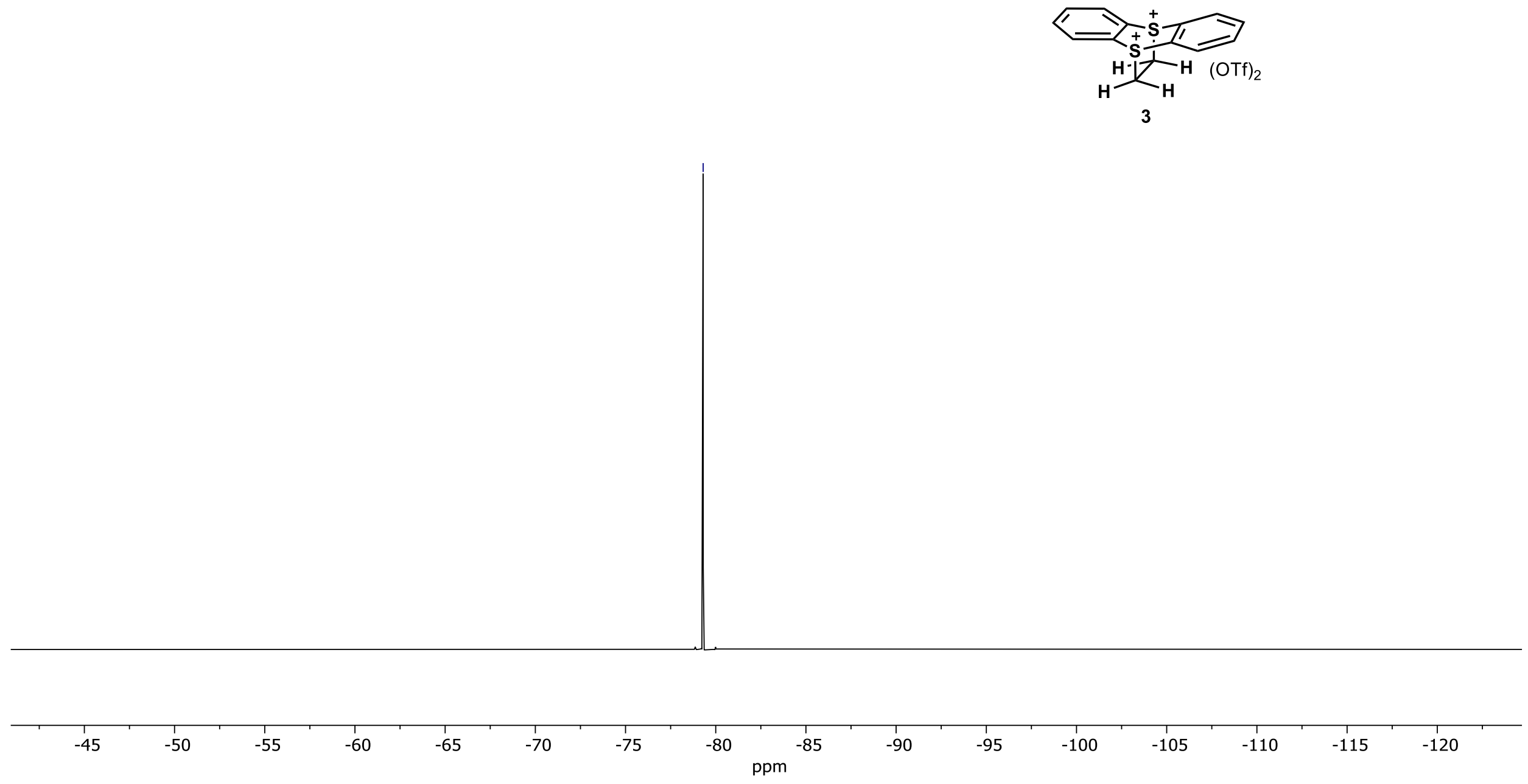
${ }^{1} \mathrm{H}$ NMR of spiro[cyclopropane-1,3'-indolin]-2'-one (5)

$\mathrm{CDCl}_{3}, 298 \mathrm{~K}$

L

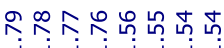
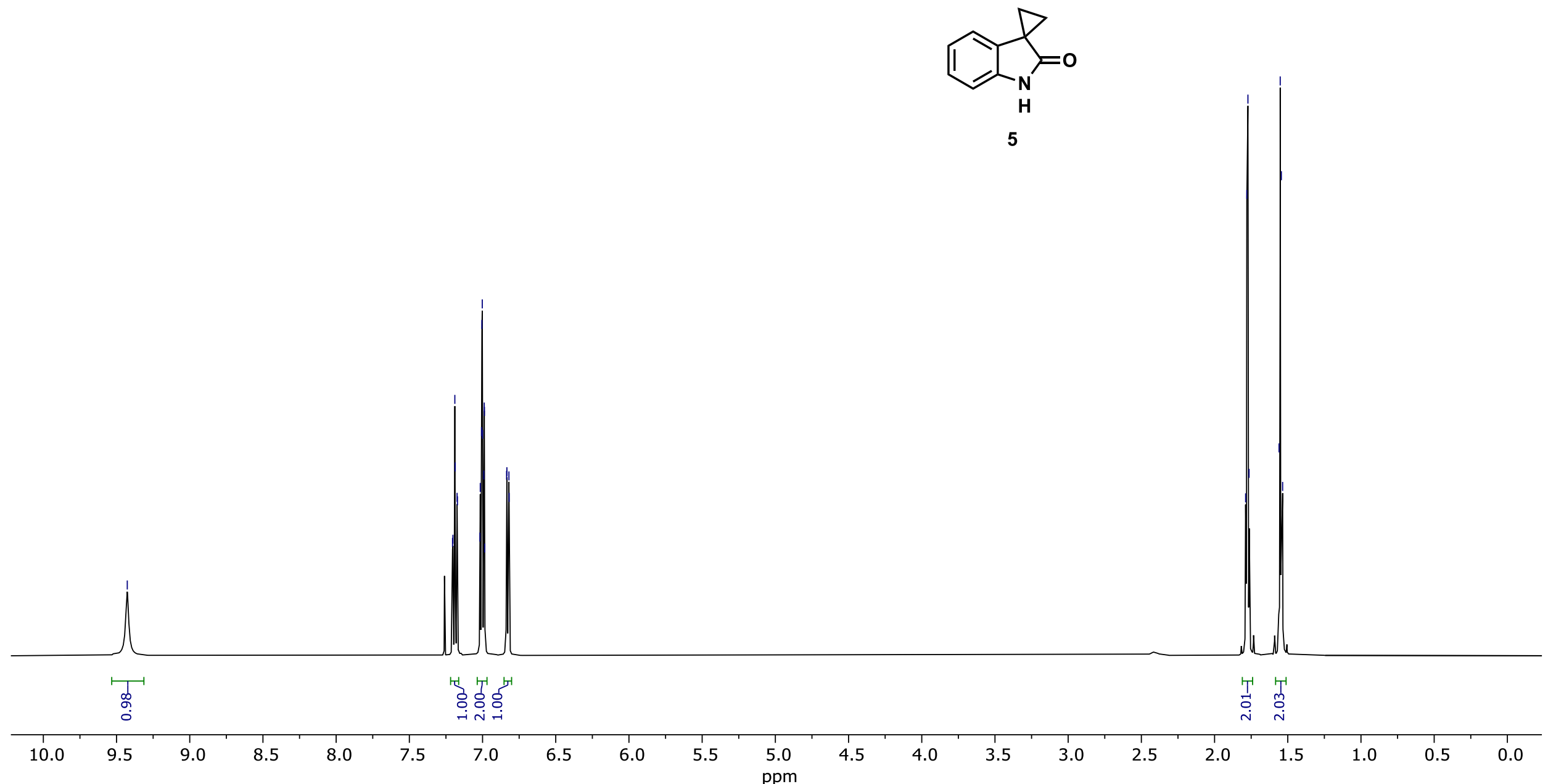
${ }^{13} \mathrm{C}$ NMR of spiro[cyclopropane-1,3'-indolin]-2'-one (5)

$\mathrm{CDCl}_{3}, 298 \mathrm{~K}$

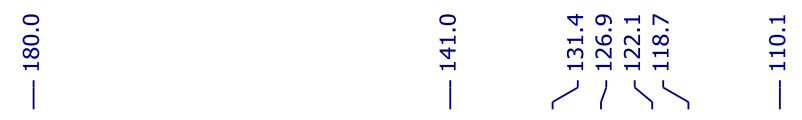

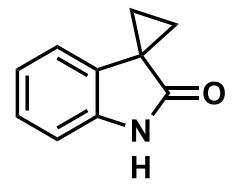

5

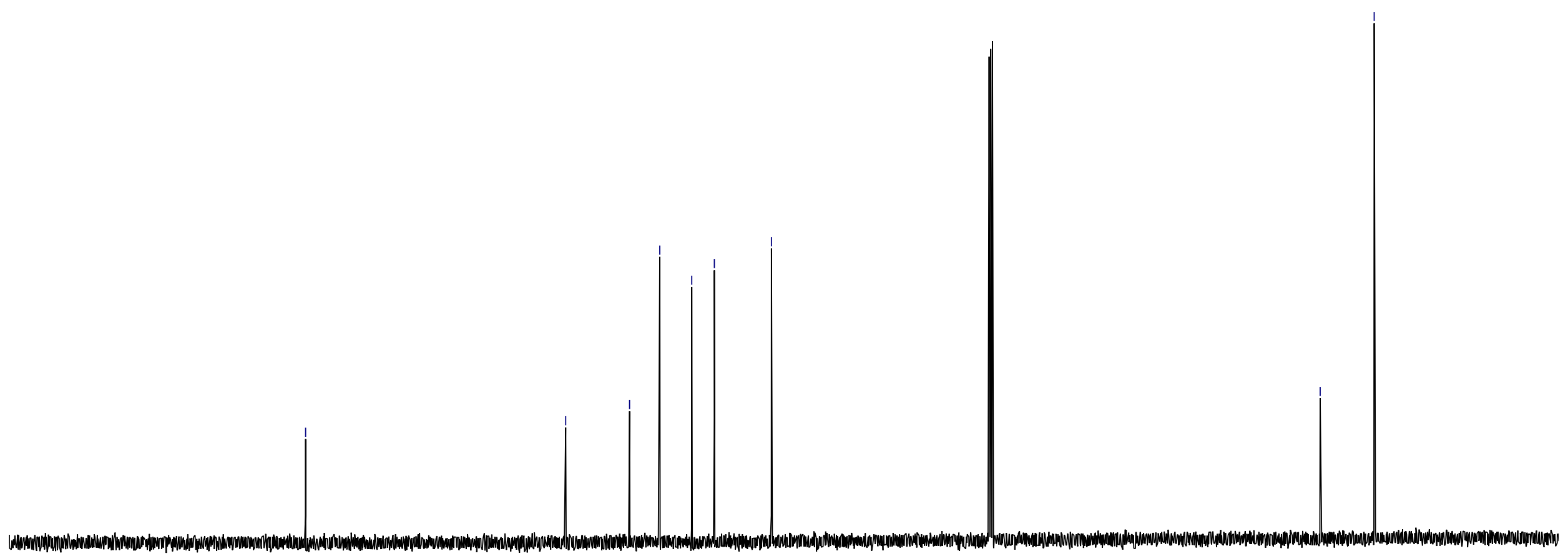

$170 \quad 160$

150

$140 \quad 130$

120

110

100

90

80

70

60

50

40

30

20

10 
${ }^{1} \mathrm{H}$ NMR of methyl ( \pm )-4-tosylmorpholine-3-carboxylate (7)

$\mathrm{CDCl}_{3}, 298 \mathrm{~K}$

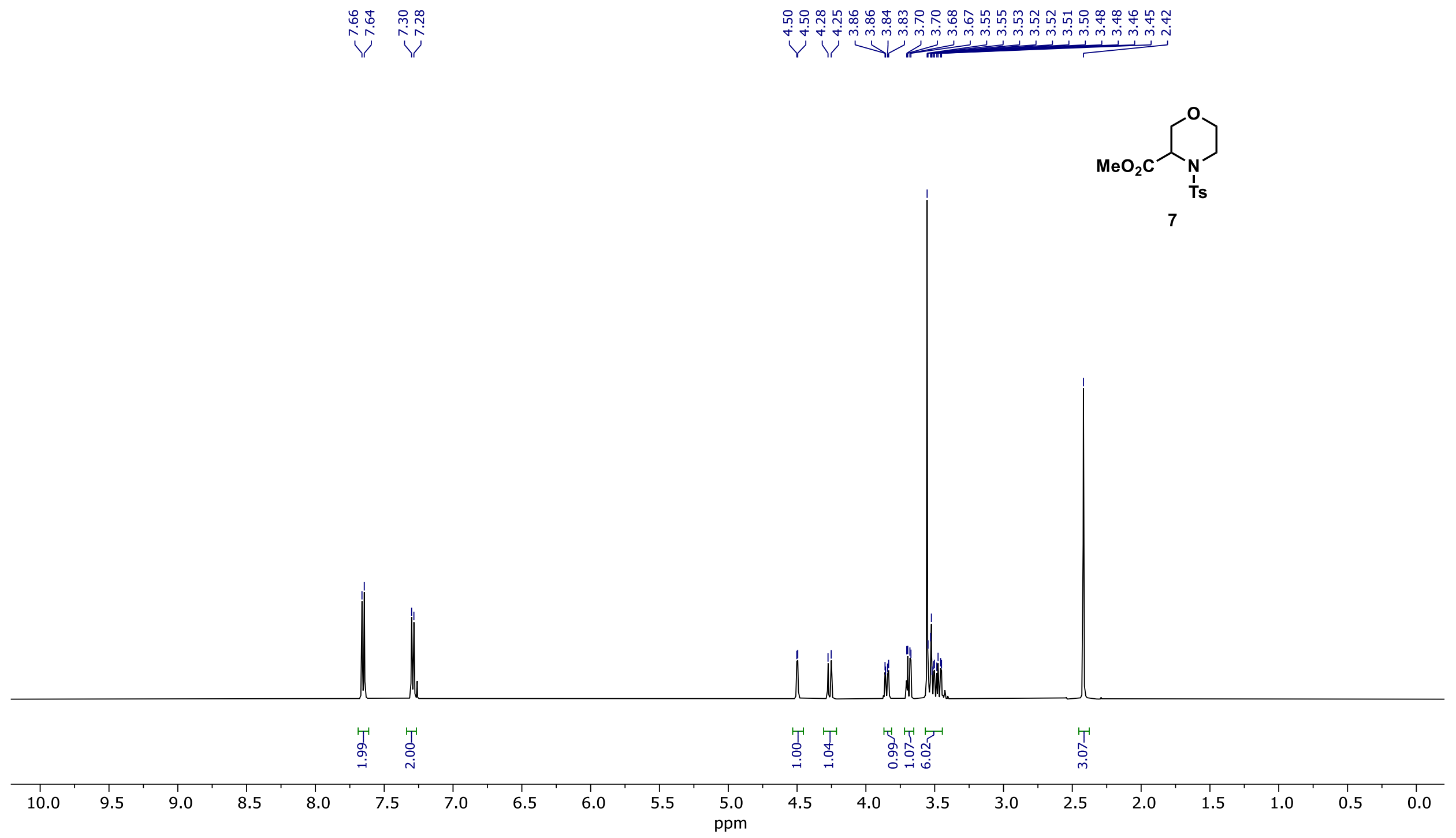


${ }^{1} \mathrm{H}$ NMR of ethyl ( \pm )-2-phenyl-1-tosylazetidine-2-carboxylate (9)

$\mathrm{CDCl}_{3}, 298 \mathrm{~K}$

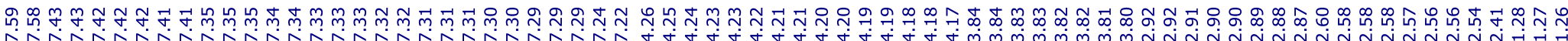

的

9

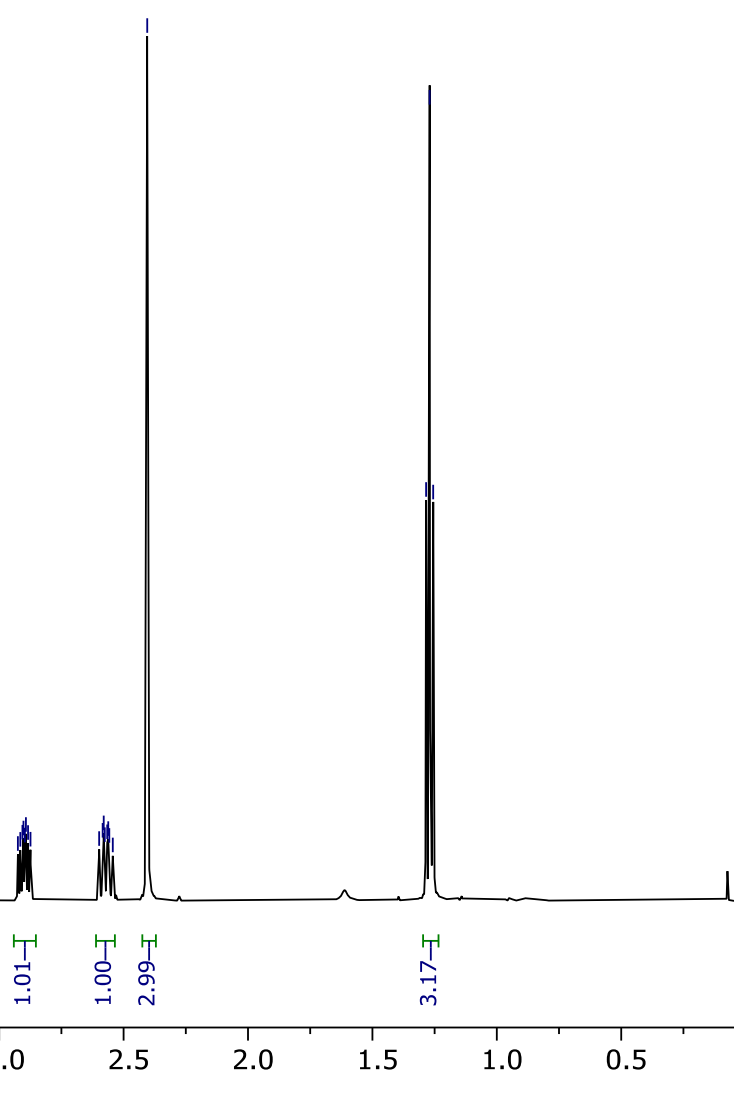




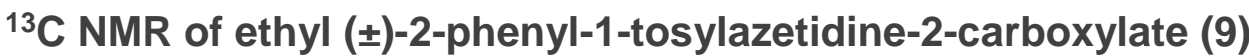

$\mathrm{CDCl}_{3}, 298 \mathrm{~K}$

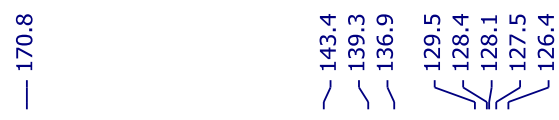

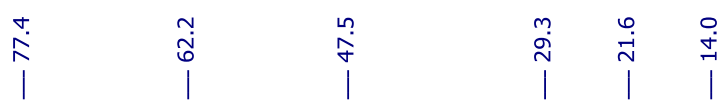
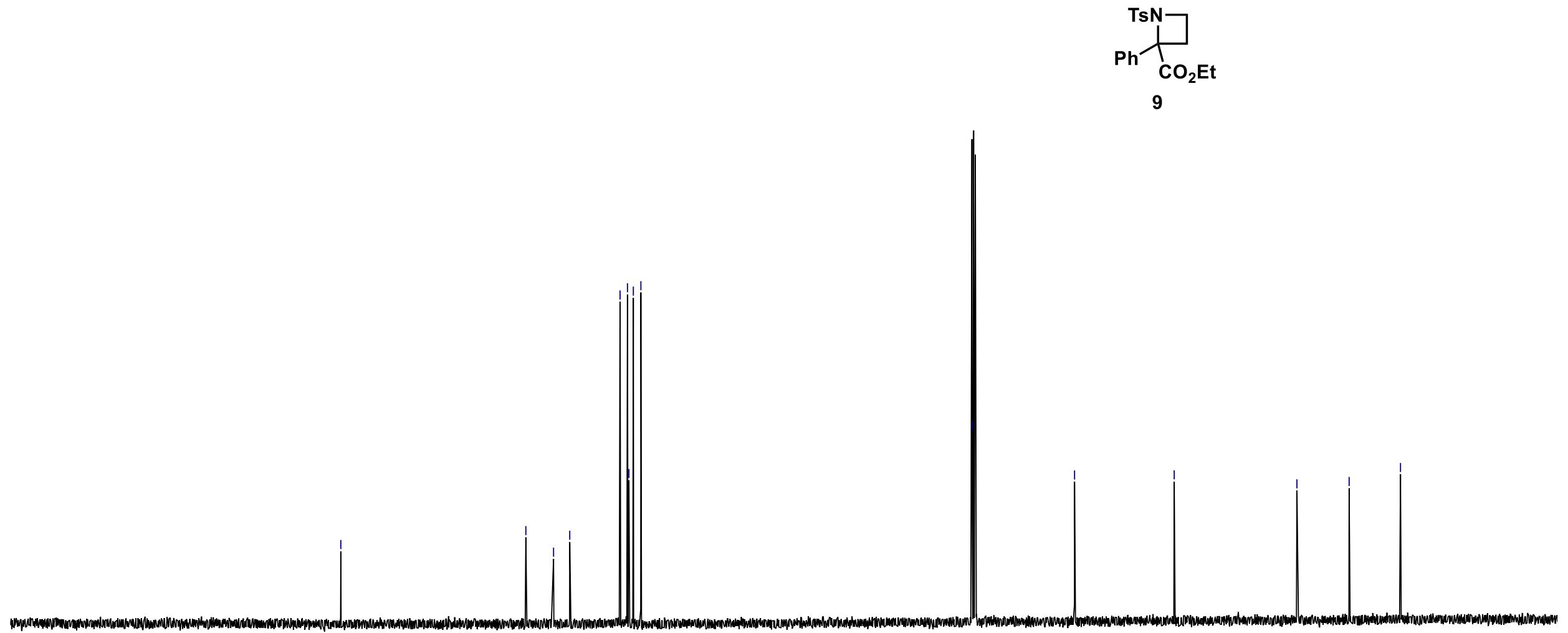

210200 $190 \quad 180$ $170 \quad 160$ 150 140 130 120 $110 \quad 100$ 90 
${ }^{1} \mathrm{H}$ NMR of ( \pm )-3-tosyl-7-oxa-3-azabicyclo[4.1.0]heptane (11)

$\mathrm{CDCl}_{3}, 298 \mathrm{~K}$

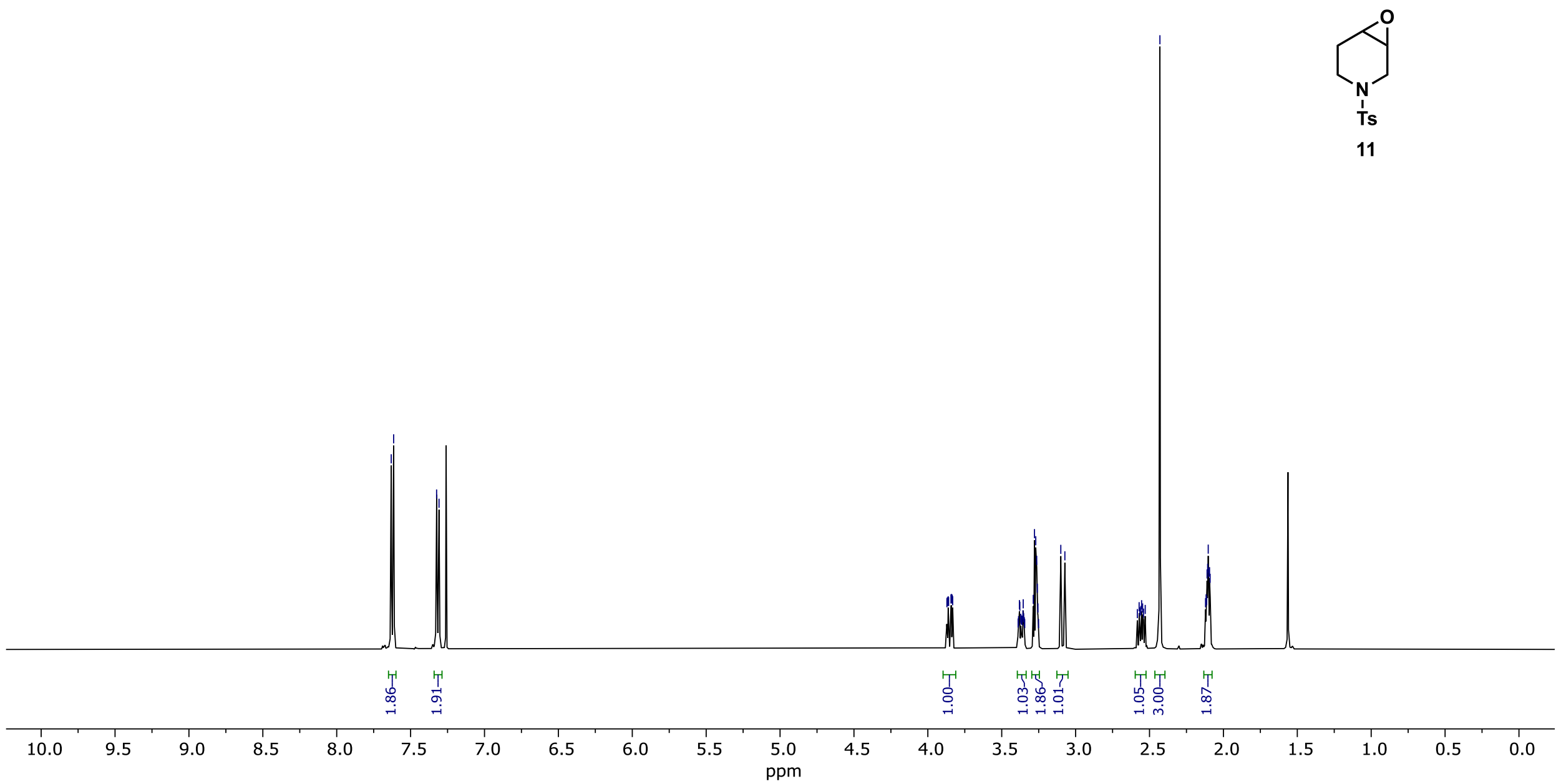




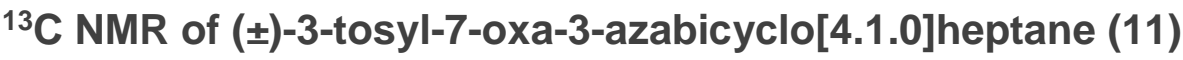

$\mathrm{CDCl}_{3}, 298 \mathrm{~K}$

\}

นึ่

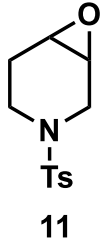

(n)

$220 \quad 210$

200

$190 \quad 180$

150

130

$120 \quad 110$

100

80

$70 \quad 60$

50

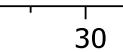

$20 \quad 10 \quad 0$ 


\section{${ }^{1} \mathrm{H}$ NMR of 9-vinyl-9H-pyrido[3,4-b]indole (12)}

DMSO- $\mathrm{d}_{6}, 298 \mathrm{~K}$

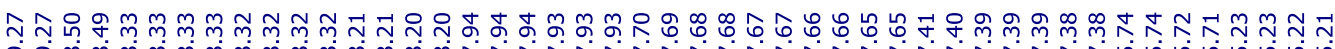

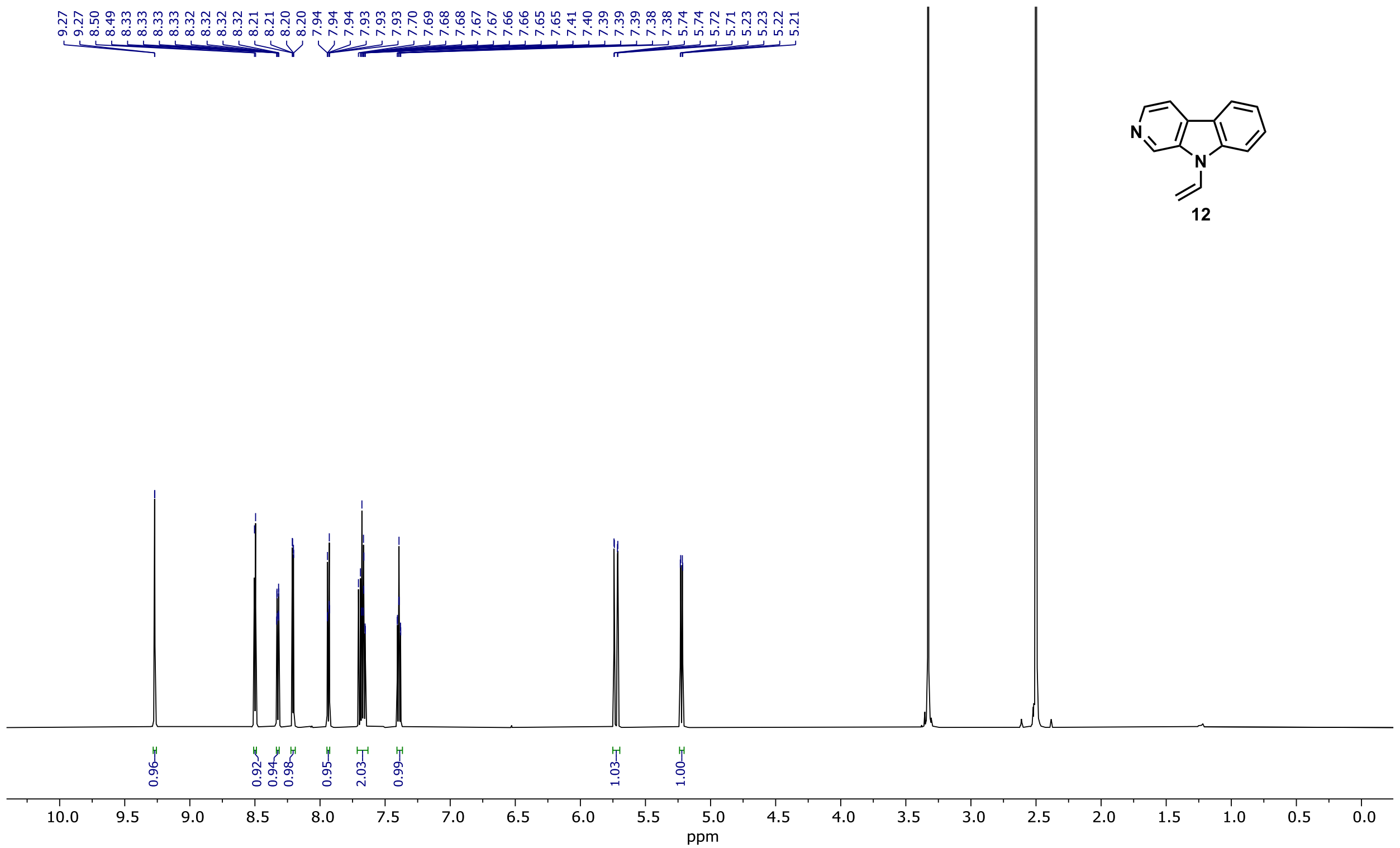


SUPPORTING INFORMATION

S76

${ }^{13} \mathrm{C}$ NMR of 9 -vinyl-9H-pyrido[3,4-b]indole (12)

DMSO-d $6,298 \mathrm{~K}$
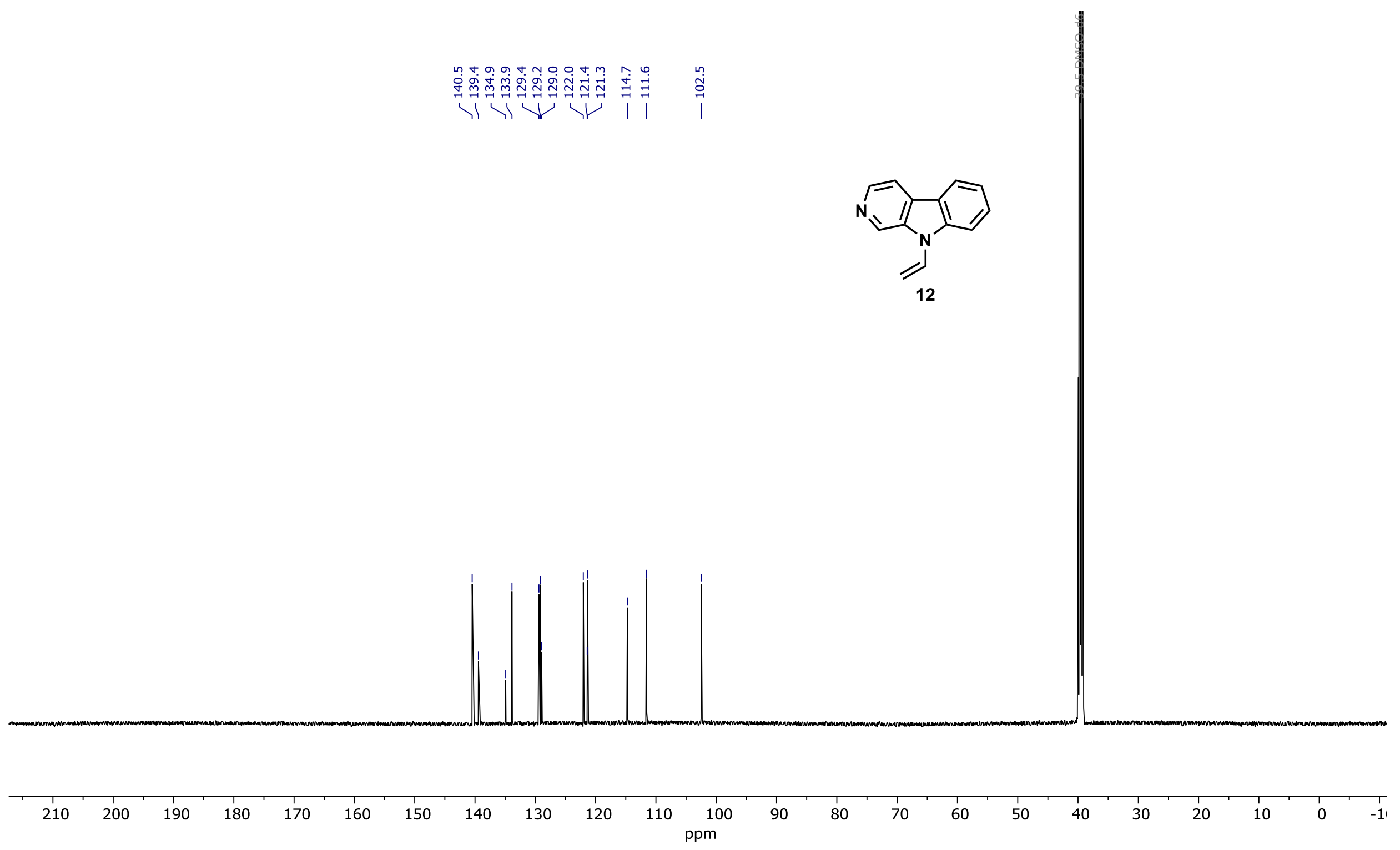
${ }^{1} \mathrm{H}$ NMR of 5-nitro-1-vinyl- $1 H$-indole (13)

$\mathrm{CDCl}_{3}, 298 \mathrm{~K}$

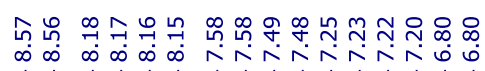

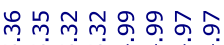

$i_{\infty}^{\infty} \underbrace{\infty} \underbrace{\infty}$
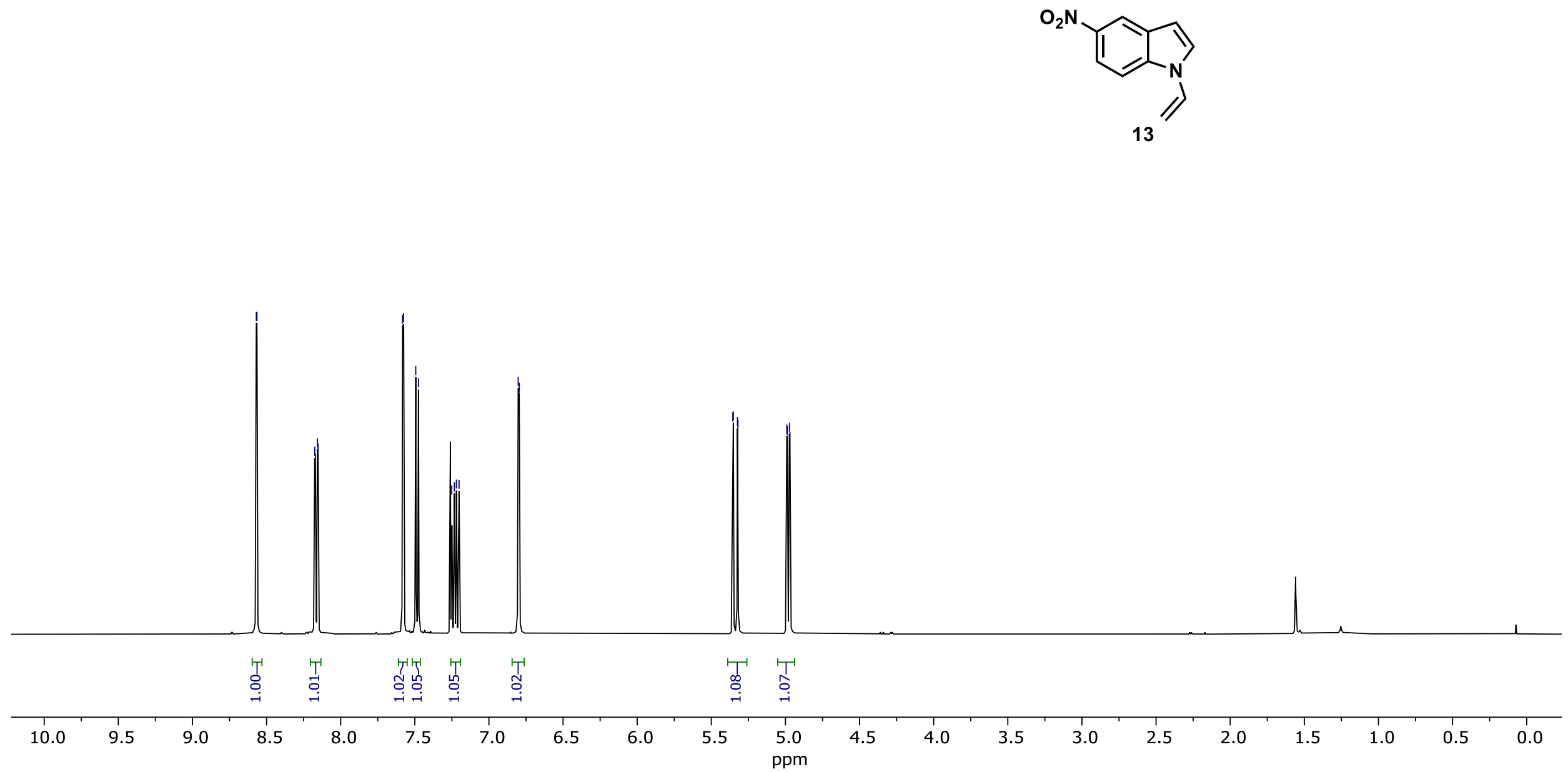
${ }^{13} \mathrm{C}$ NMR of 5-nitro-1-vinyl-1H-indole (13)

$\mathrm{CDCl}_{3}, 298 \mathrm{~K}$

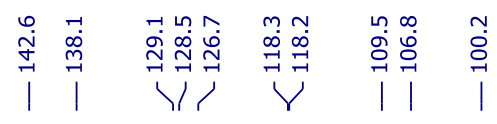

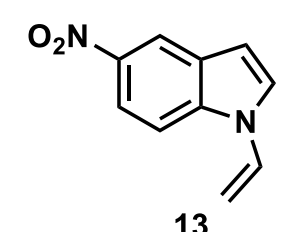

13

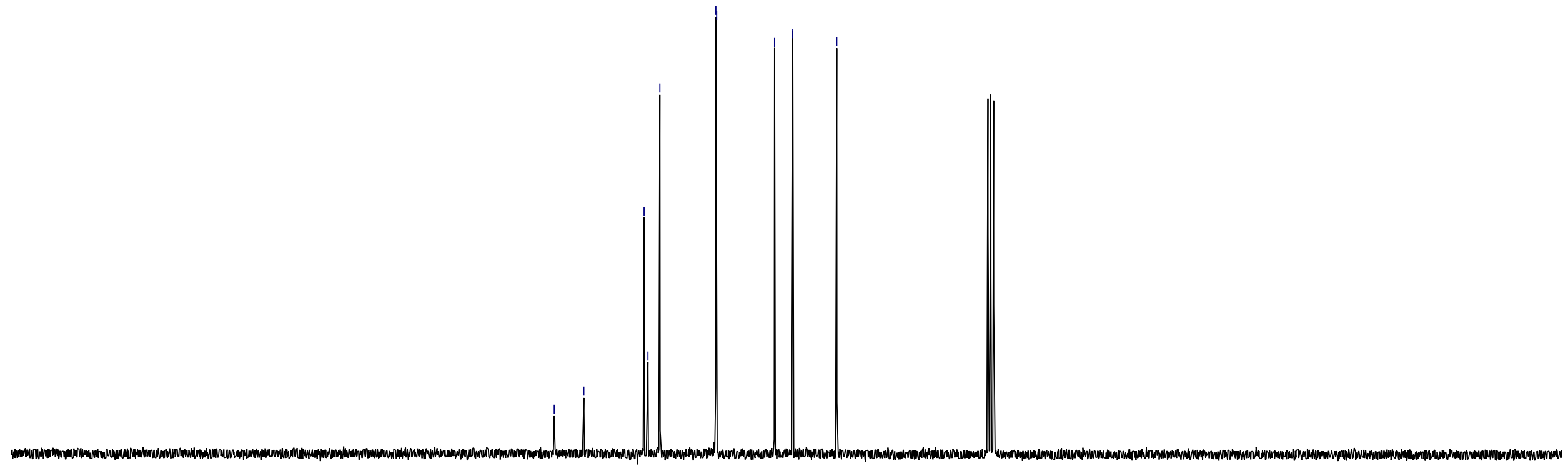

$\begin{array}{lllllllllllllllllllllllll}220 & 210 & 200 & 190 & 180 & 170 & 160 & 150 & 140 & 130 & 120 & 110 & 100 & 90 & 80 & 70 & 60 & 50 & 40 & 30 & 20 & 10 & 0 & 10\end{array}$


${ }^{1} \mathrm{H}$ NMR of 1-vinyl-1 $H$-indole-3-carbaldehyde (14)

$\mathrm{CDCl}_{3}, 298 \mathrm{~K}$

i

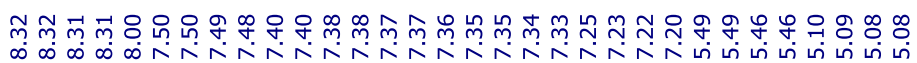

舟

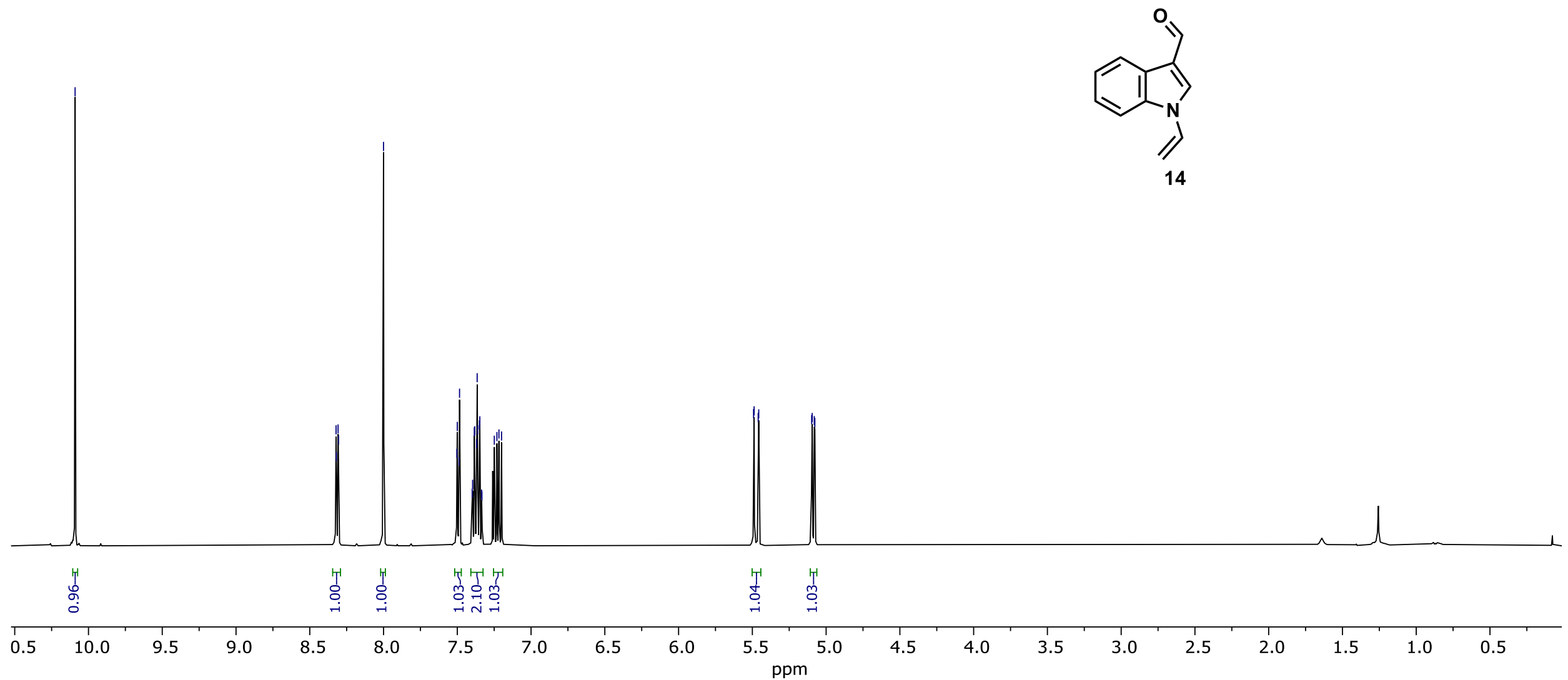


${ }^{13} \mathrm{C}$ NMR of 1 -vinyl-1 $H$-indole-3-carbaldehyde (14)

$\mathrm{CDCl}_{3}, 298 \mathrm{~K}$

|
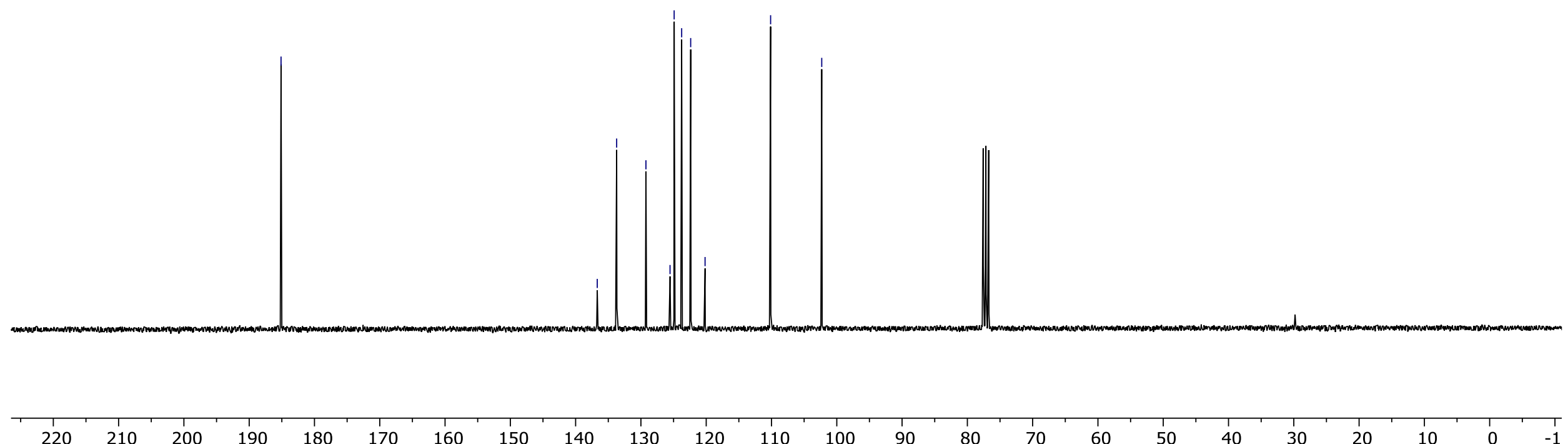

$\begin{array}{lll}190 & 190 \quad 180\end{array}$
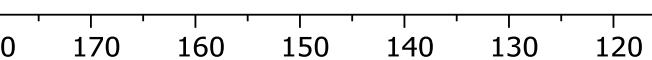
${ }^{1} \mathrm{H}$ NMR of ethyl 3-amino-1-vinyl-1H-pyrazole-4-carboxylate (15-I)

$\mathrm{CD}_{2} \mathrm{Cl}_{2}, 298 \mathrm{~K}$

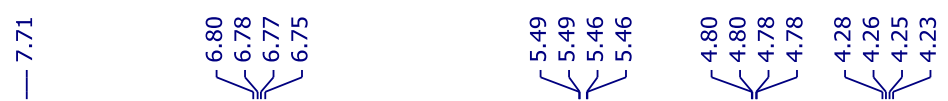

$\stackrel{m}{m} \underset{m}{m} \vec{m}-\vec{m}$

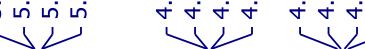

$\checkmark$
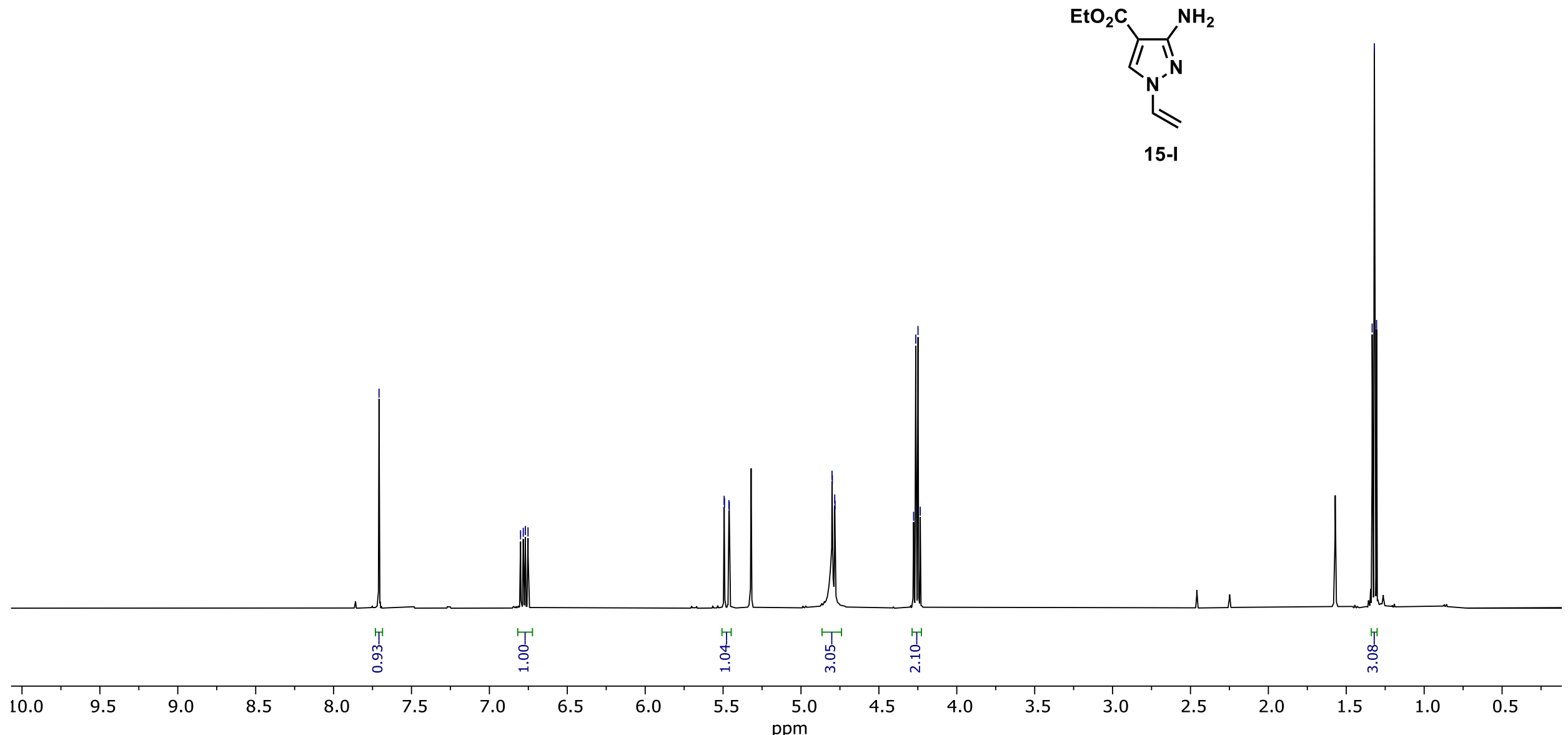
${ }^{13} \mathrm{C}$ NMR of ethyl 3-amino-1-vinyl-1H-pyrazole-4-carboxylate (15-I)

$\mathrm{CD}_{2} \mathrm{Cl}_{2}, 298 \mathrm{~K}$

I

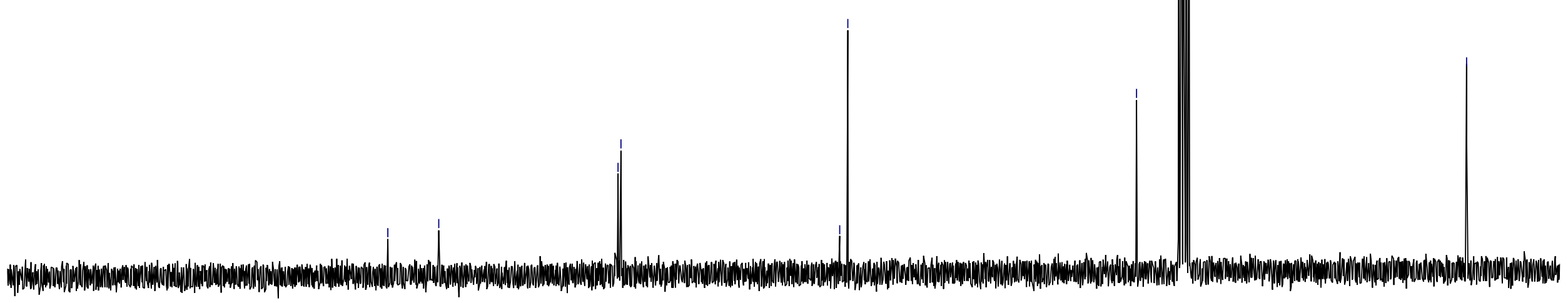


${ }^{1} \mathrm{H}$ NMR of ethyl 5-amino-1-vinyl-1H-pyrazole-4-carboxylate (15-II)

$\mathrm{CD}_{2} \mathrm{Cl}_{2}, 298 \mathrm{~K}$

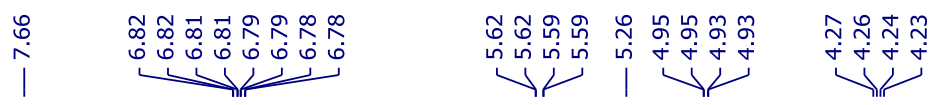

$\mathrm{EtO}_{2} \mathrm{C}$

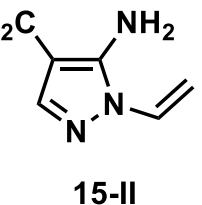

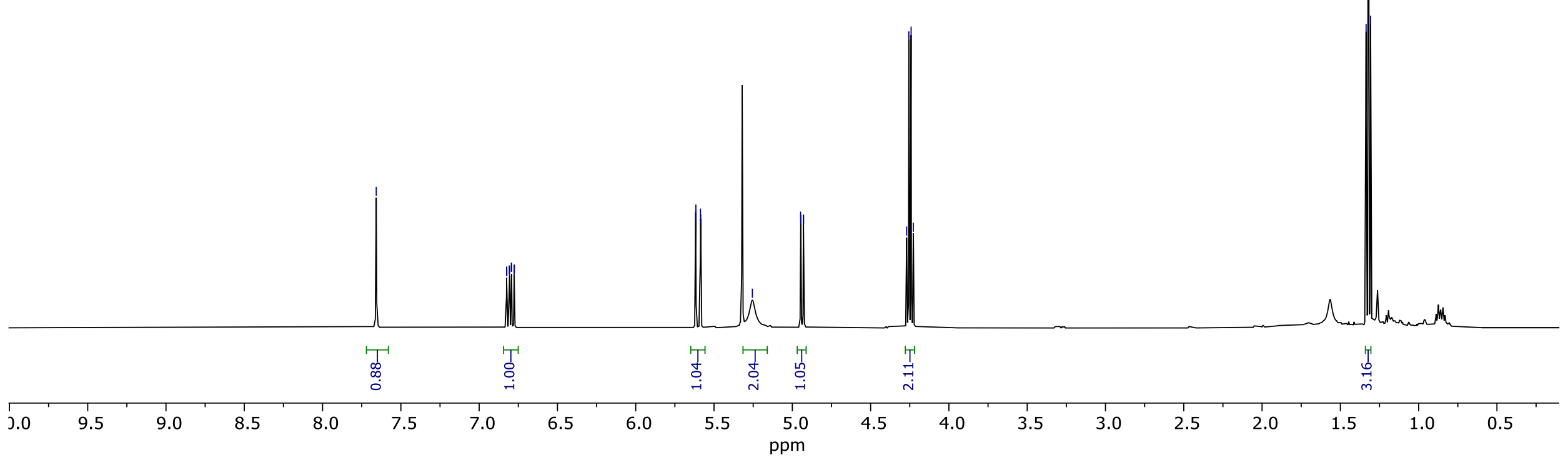


${ }^{13} \mathrm{C}$ NMR of ethyl 5-amino-1-vinyl-1H-pyrazole-4-carboxylate (15-II)

$\mathrm{CD}_{2} \mathrm{Cl}_{2}, 298 \mathrm{~K}$

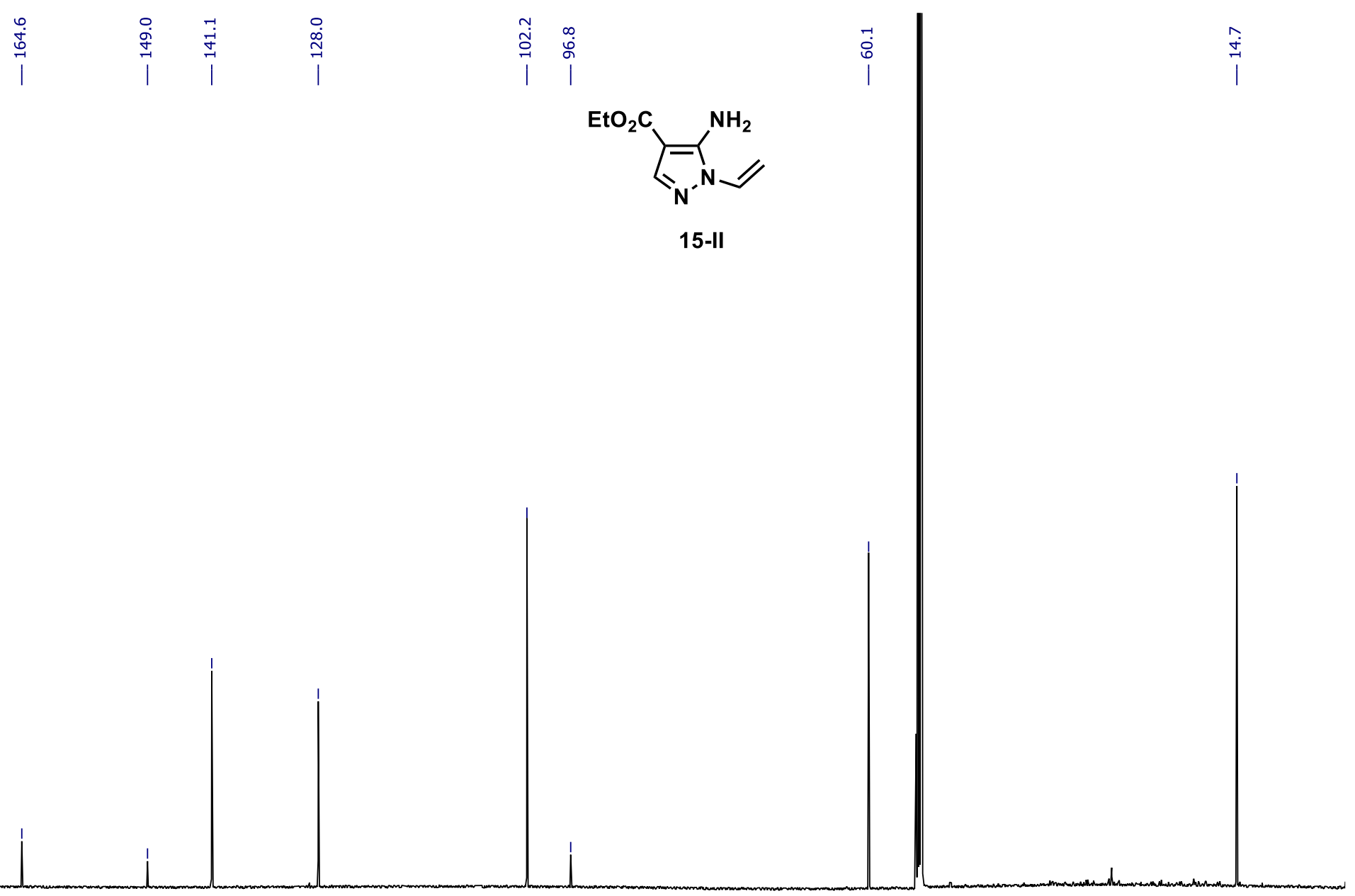


${ }^{1} \mathrm{H}$ NMR of 4-bromo-3,5-dimethyl-1-vinyl-1 H-pyrazole (16)

$\mathrm{CDCl}_{3}, 298 \mathrm{~K}$

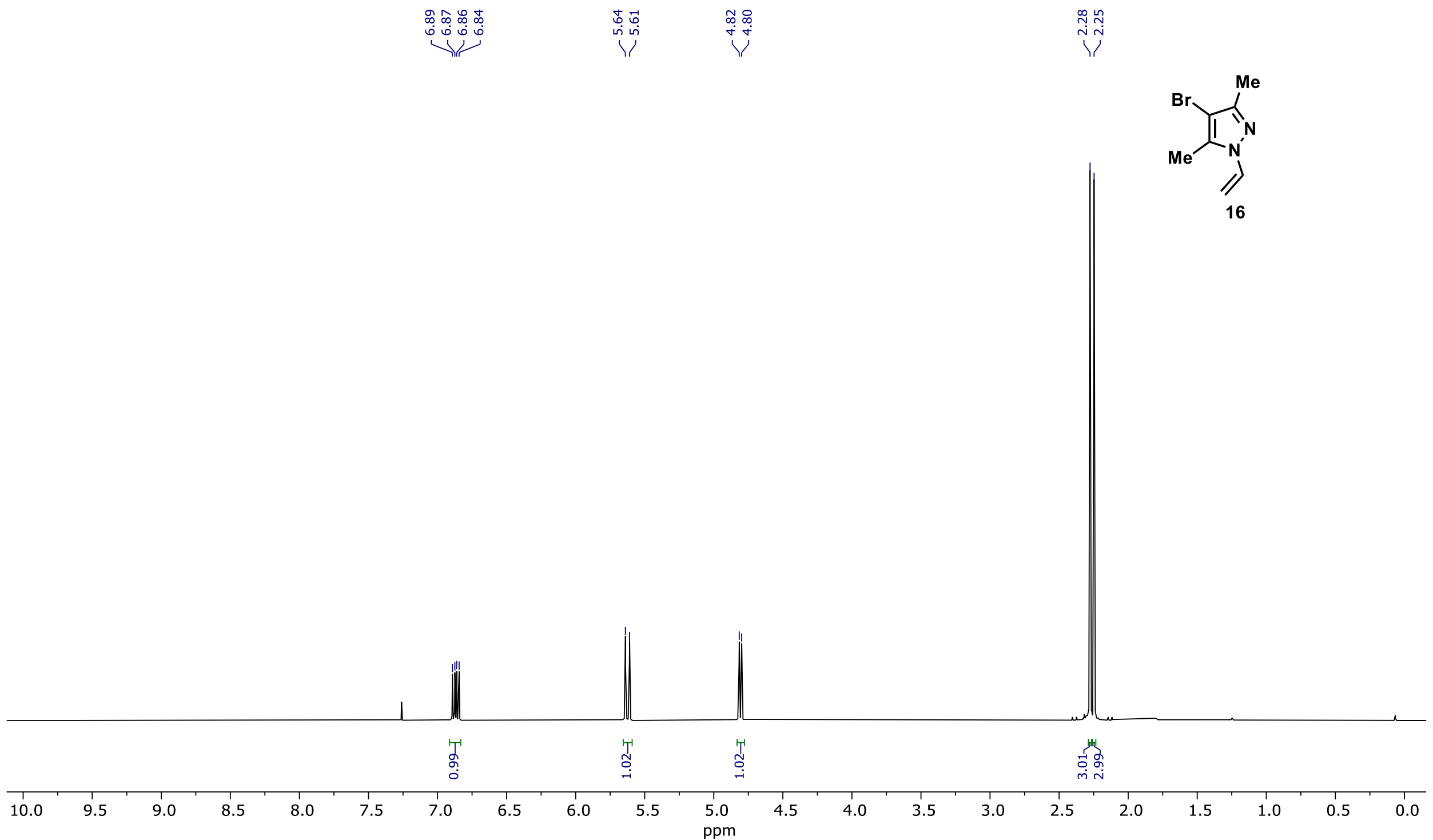


${ }^{13} \mathrm{C}$ NMR of 4-bromo-3,5-dimethyl-1-vinyl-1 H-pyrazole (16)

$\mathrm{CDCl}_{3}, 298 \mathrm{~K}$

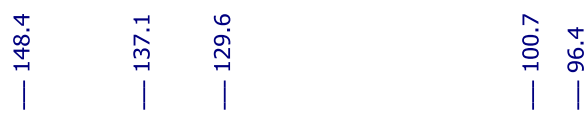
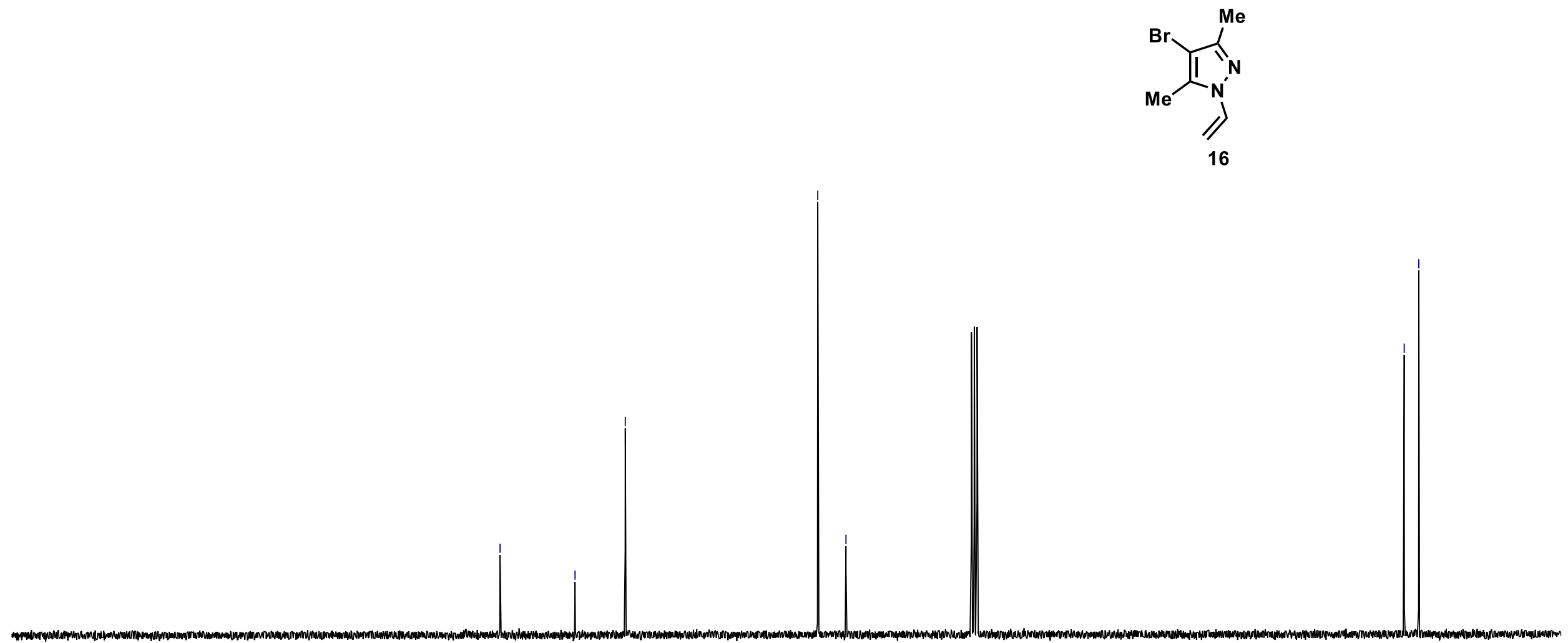

220

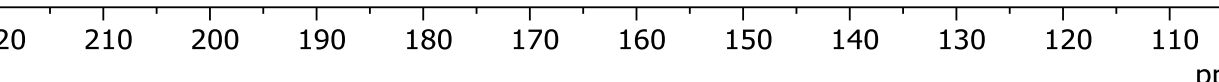
ppm 
${ }^{1} \mathrm{H}$ NMR of methyl 1-vinyl-1H-1,2,4-triazole-3-carboxylate (17)

$\mathrm{CDCl}_{3}, 298 \mathrm{~K}$

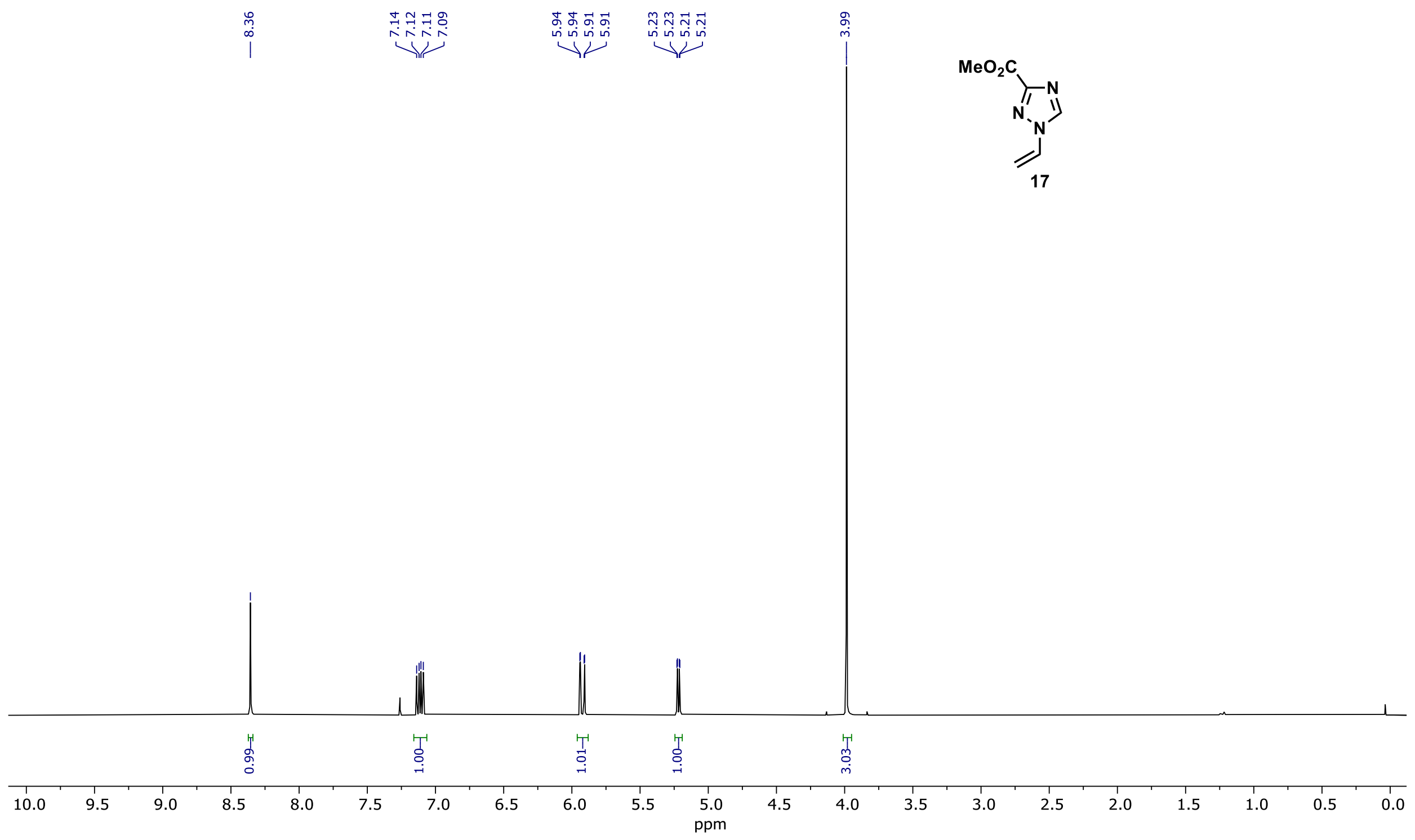


${ }^{13} \mathrm{C}$ NMR of methyl 1-vinyl-1 H-1,2,4-triazole-3-carboxylate (17)

$\mathrm{CDCl}_{3}, 298 \mathrm{~K}$

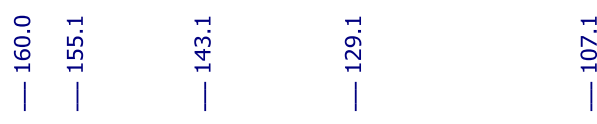

$\mathrm{MeO}_{2} \mathrm{C}$

$$
{ }_{17}^{N}{ }^{-N}
$$

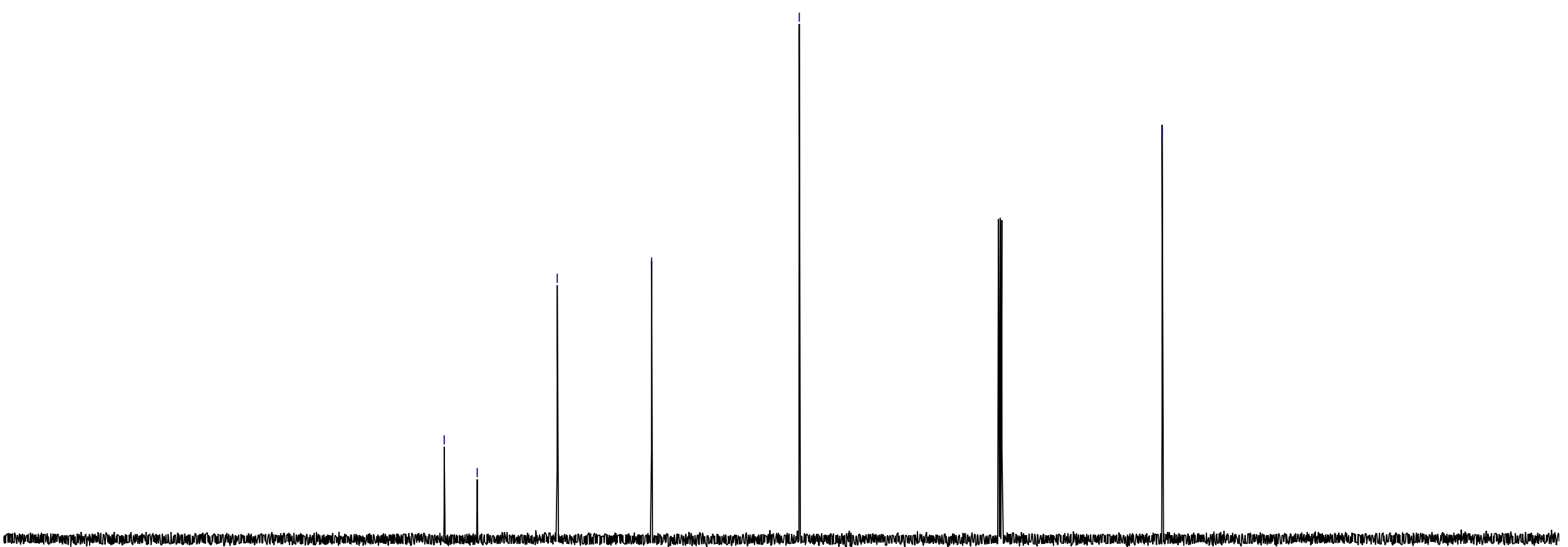


${ }^{1} \mathrm{H}$ NMR of 1-vinylpyridin-4(1H)-one (18)

$\mathrm{CDCl}_{3}, 298 \mathrm{~K}$
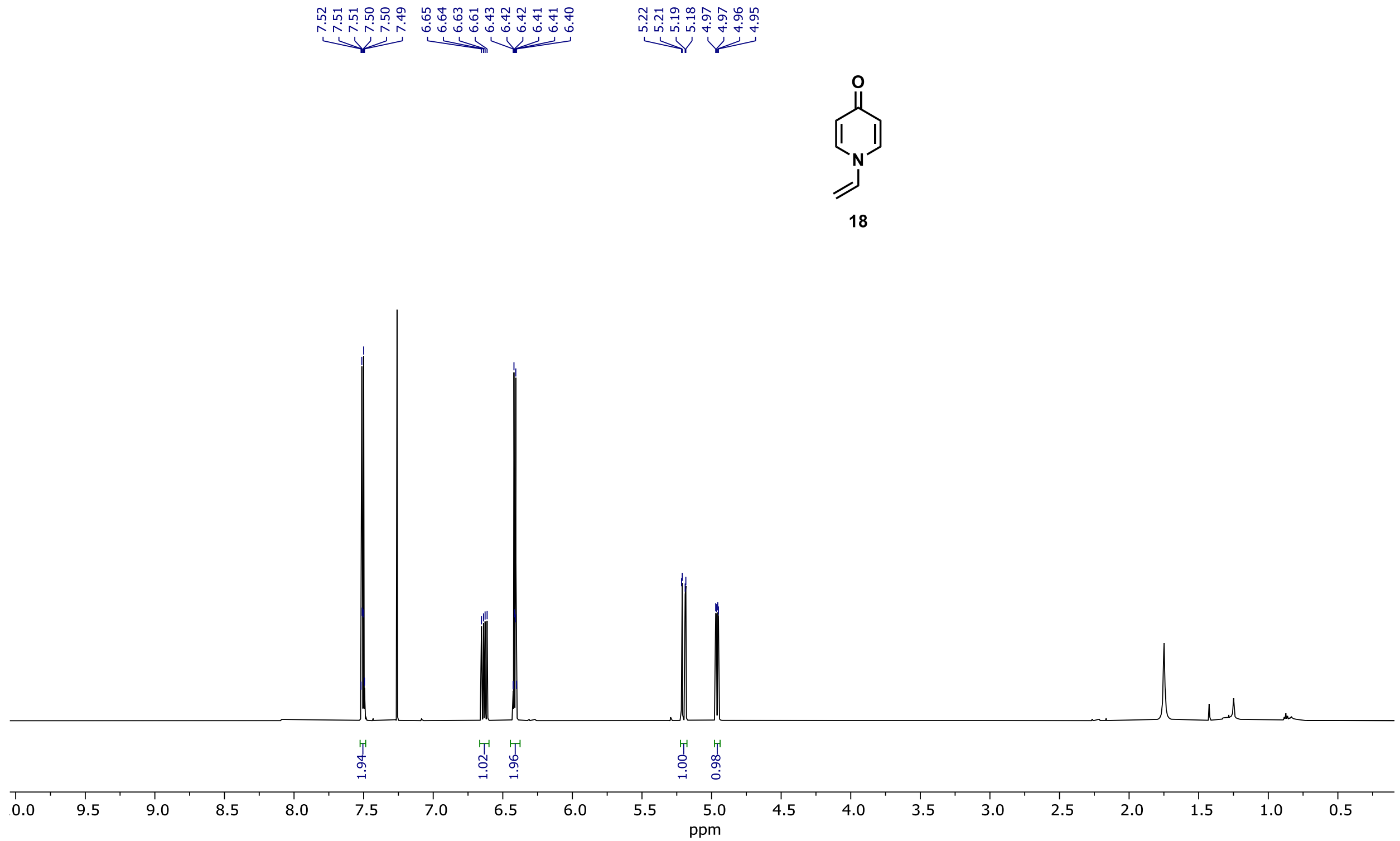
${ }^{13} \mathrm{C}$ NMR of 1 -vinylpyridin-4(1H)-one (18)

$\mathrm{CDCl}_{3}, 298 \mathrm{~K}$ 
${ }^{1} \mathrm{H}$ NMR of 4-(4-fluorophenyl)-1-vinyl-1 H-imidazole (19)

$\mathrm{CD}_{2} \mathrm{Cl}_{2}, 298 \mathrm{~K}$

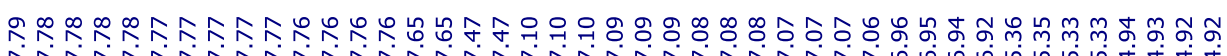

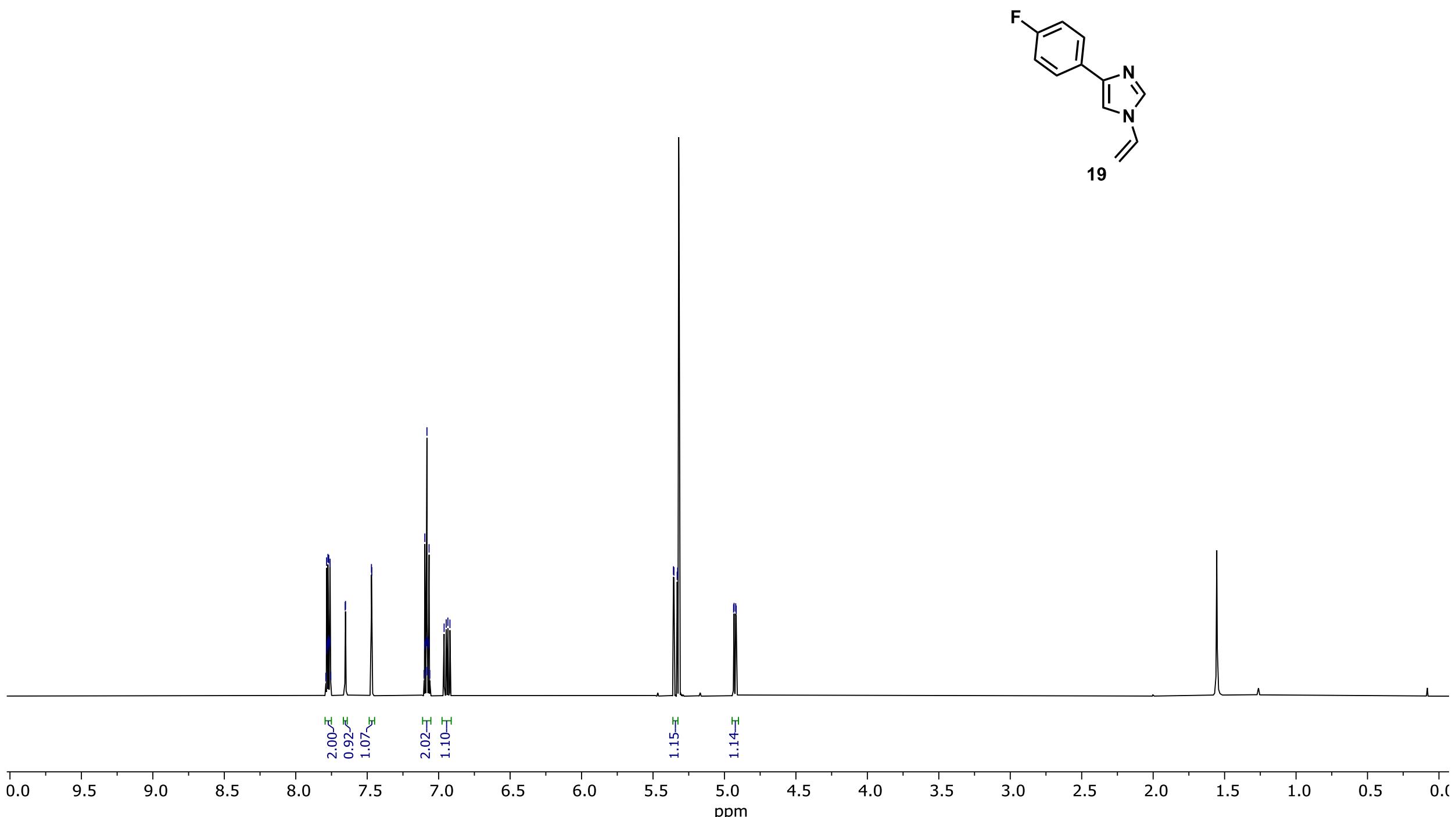


${ }^{13} \mathrm{C}$ NMR of 4-(4-fluorophenyl)-1-vinyl-1 H-imidazole (19)

$\mathrm{CD}_{2} \mathrm{Cl}_{2}, 298 \mathrm{~K}$

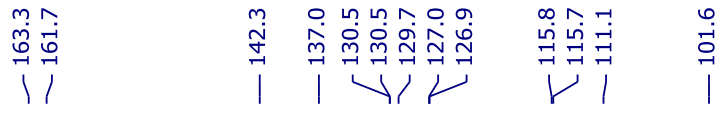

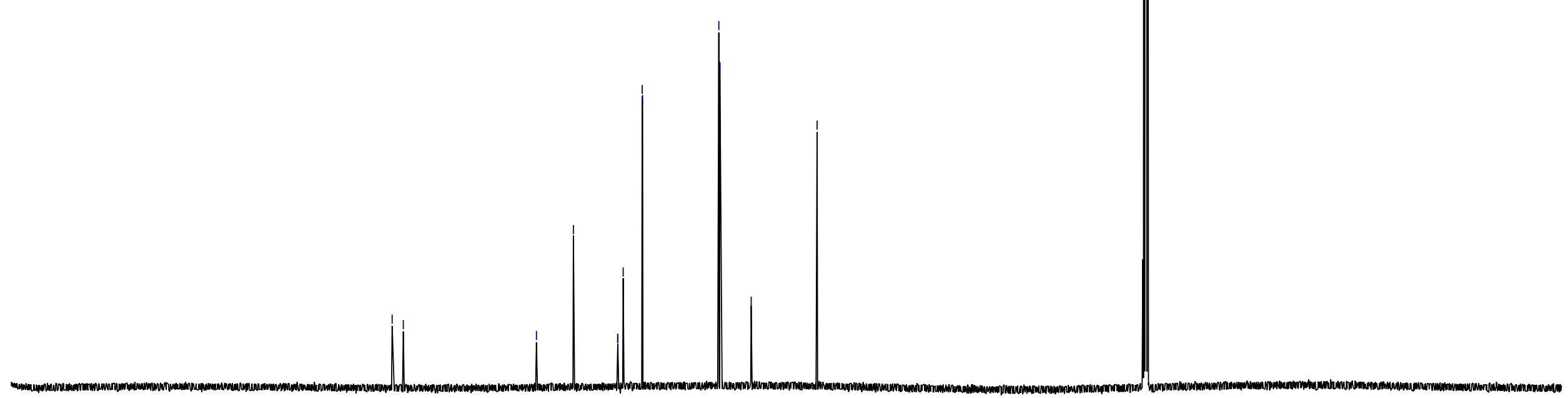


${ }^{19} \mathrm{~F}$ NMR of 4-(4-fluorophenyl)-1-vinyl-1 H-imidazole (19)

$\mathrm{CD}_{2} \mathrm{Cl}_{2}, 298 \mathrm{~K}$

$$
\begin{aligned}
& 9 \\
& \overrightarrow{0} \\
& \overrightarrow{0} \\
& i
\end{aligned}
$$

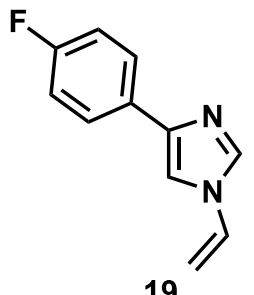

19

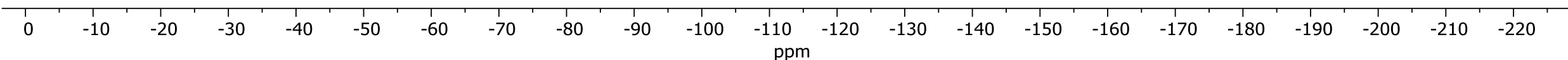


${ }^{1} \mathrm{H}$ NMR of 4-chloro-7-vinyl-7 H-pyrrolo[2,3-d]pyrimidine (20)

$\mathrm{CDCl}_{3}, 298 \mathrm{~K}$

年
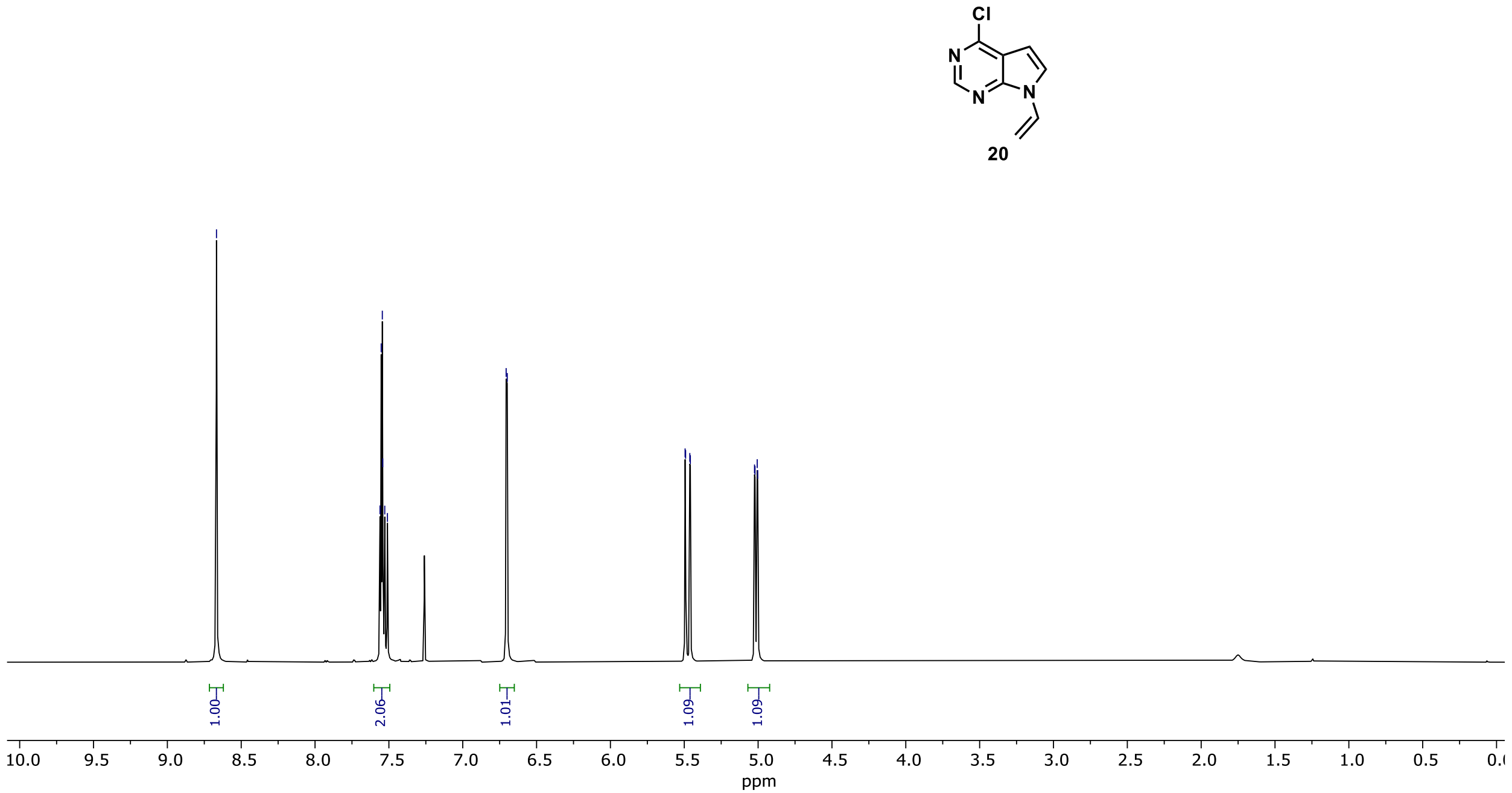
${ }^{13} \mathrm{C}$ NMR of $4-c h l o r o-7-v i n y l-7 H-p y r r o l o[2,3-d]$ pyrimidine (20)

$\mathrm{CDCl}_{3}, 298 \mathrm{~K}$

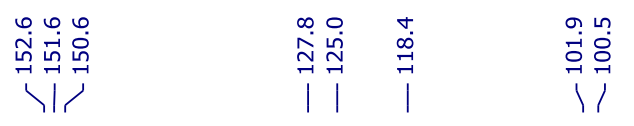
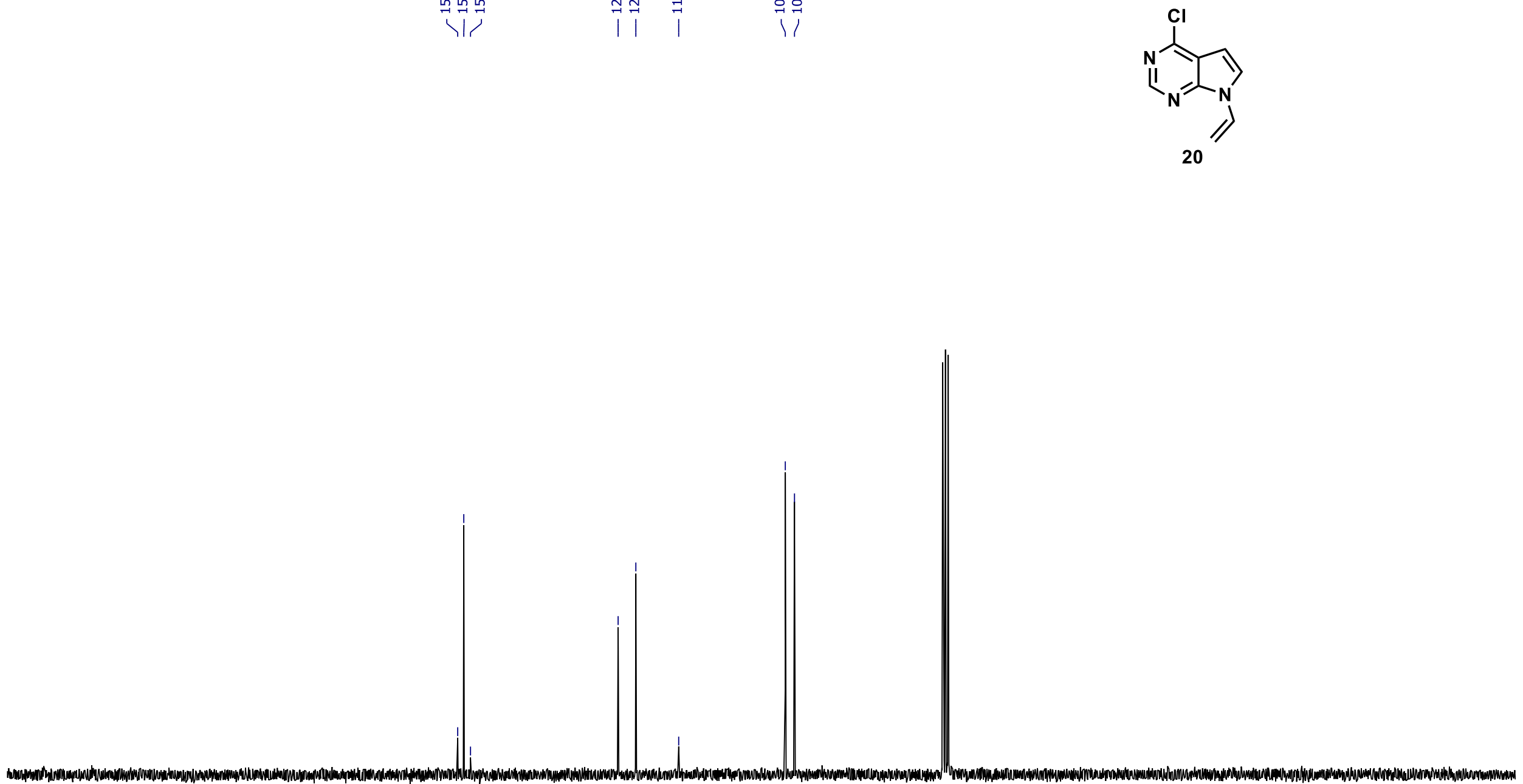
${ }^{1} \mathrm{H}$ NMR of $\mathrm{N}$-vinyl-theophylline (21)

$\mathrm{CDCl}_{3}, 298 \mathrm{~K}$

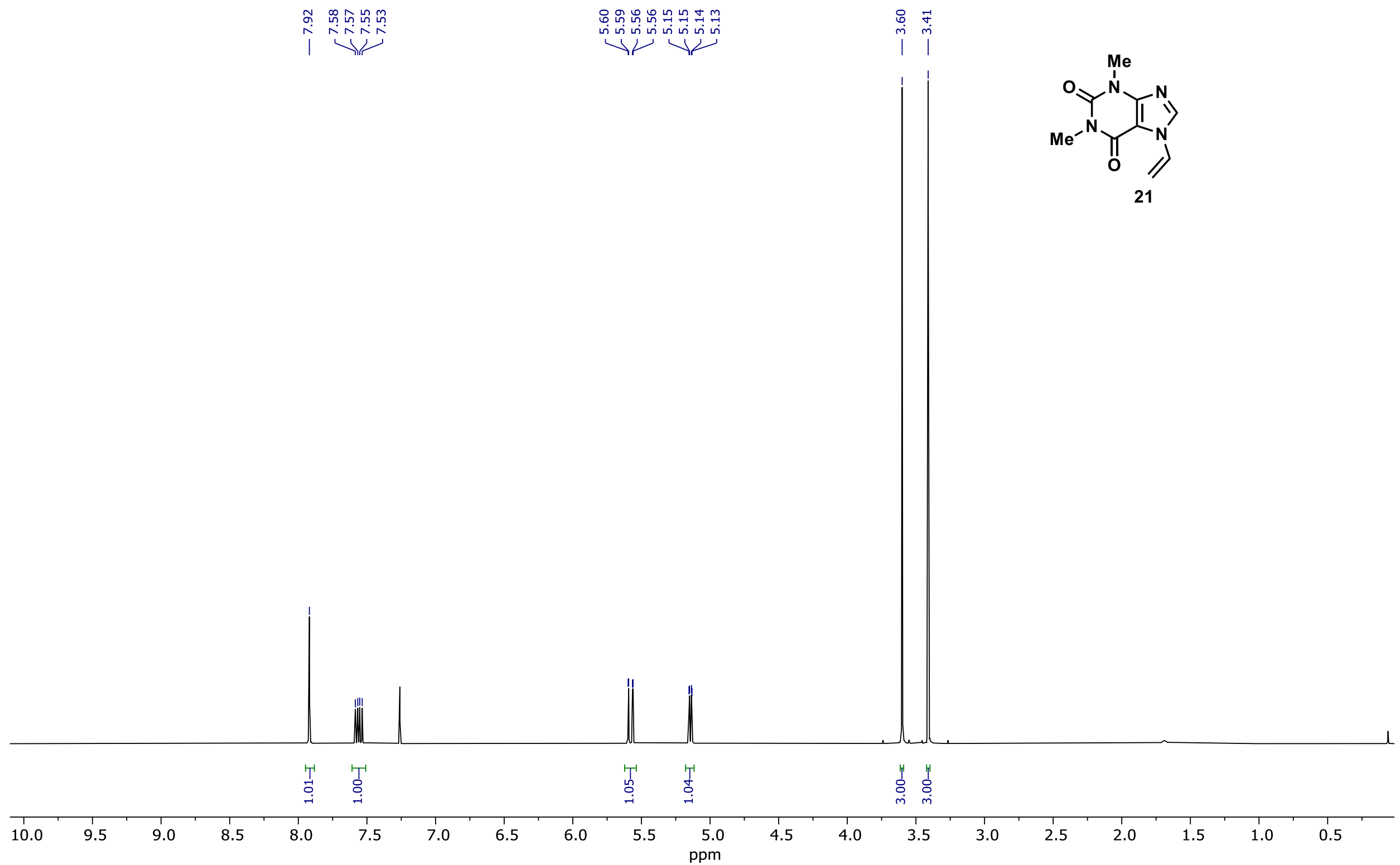


${ }^{13} \mathrm{C}$ NMR of $\mathrm{N}$-vinyl-theophylline (21)

$\mathrm{CDCl}_{3}, 298 \mathrm{~K}$

|11

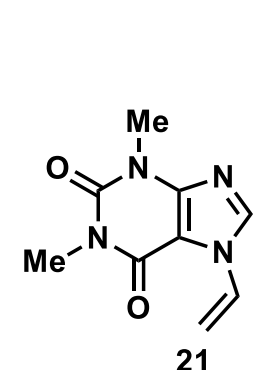


${ }^{1} \mathrm{H}$ NMR of $\mathrm{N}$-vinyl- $\mathrm{N}$-acetyl-L-tryptophan ethyl ester (22)

$\mathrm{CDCl}_{3}, 298 \mathrm{~K}$

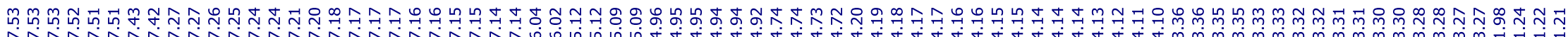
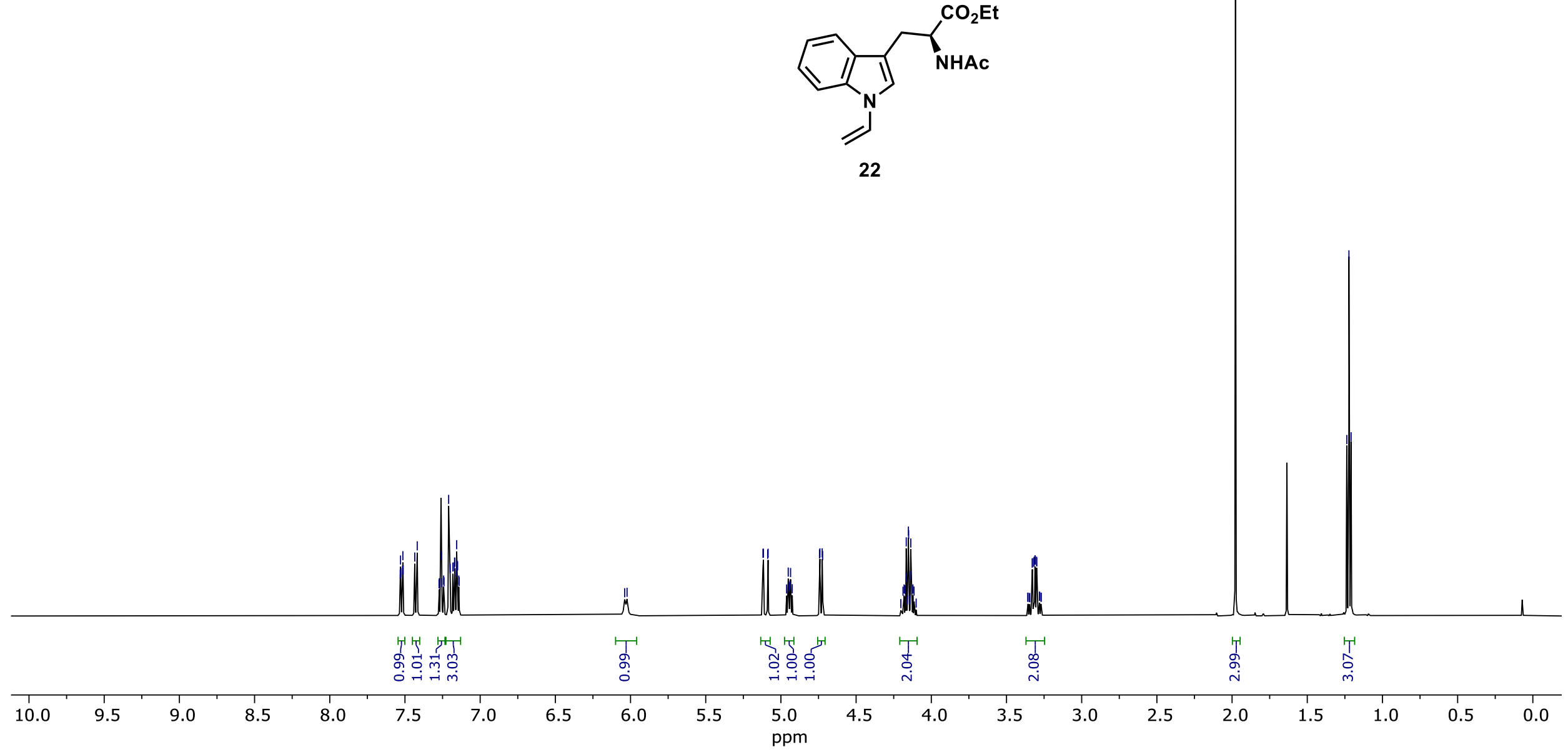
${ }^{13} \mathrm{C}$ NMR of $\mathrm{N}$-vinyl-N'-acetyl-L-tryptophan ethyl ester (22)

$\mathrm{CDCl}_{3}, 298 \mathrm{~K}$
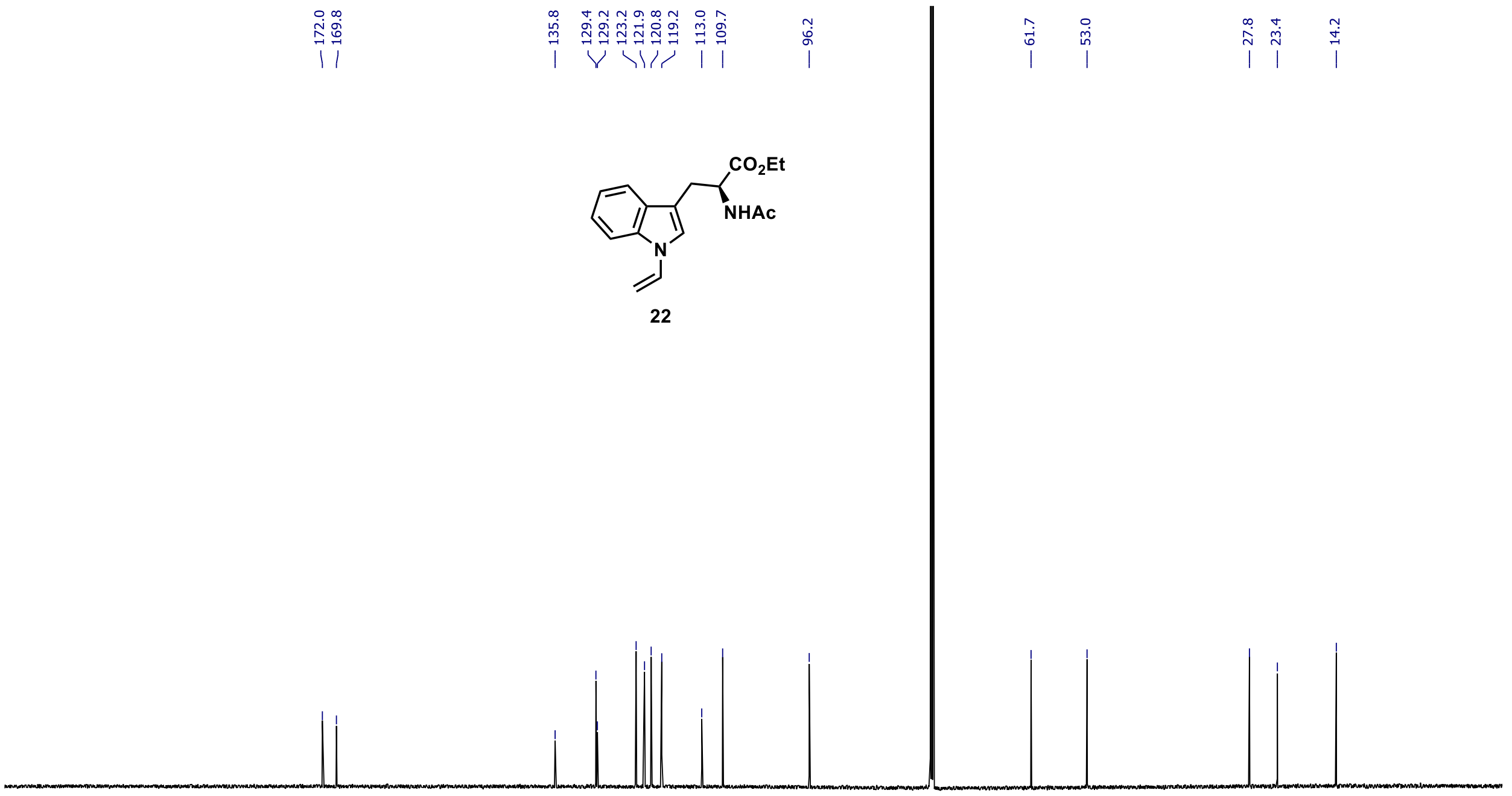

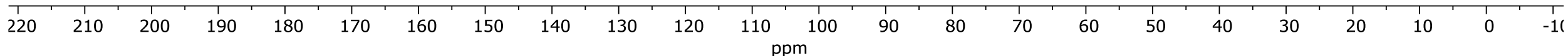


${ }^{1} \mathrm{H}$ NMR of $\mathrm{N}$-vinyl-N'-Boc-L-histidine methyl ester (23)

$\mathrm{CDCl}_{3}, 298 \mathrm{~K}$
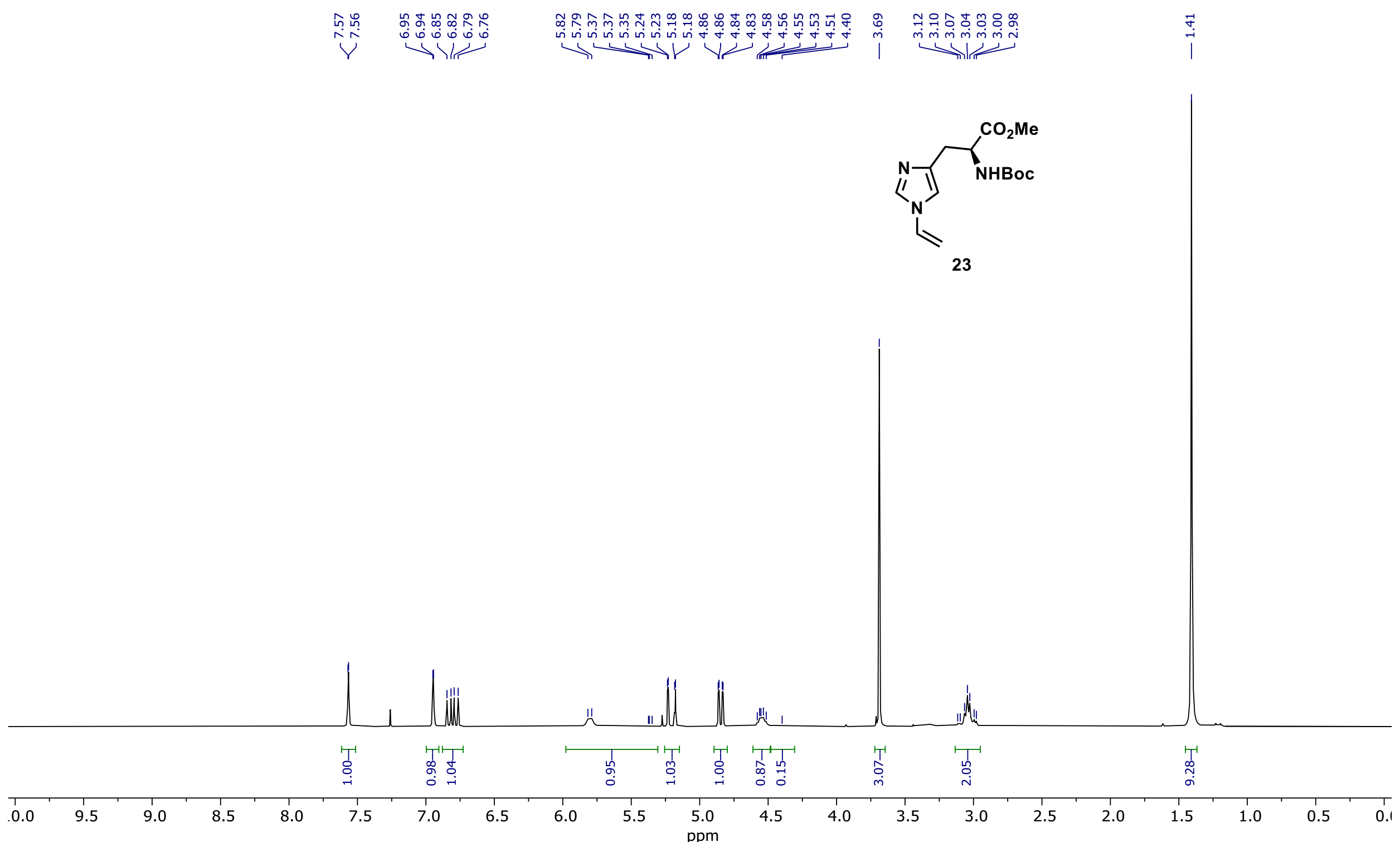
${ }^{13} \mathrm{C}$ NMR of $\mathrm{N}$-vinyl-N'-Boc-L-histidine methyl ester (23)

$\mathrm{CDCl}_{3}, 298 \mathrm{~K}$

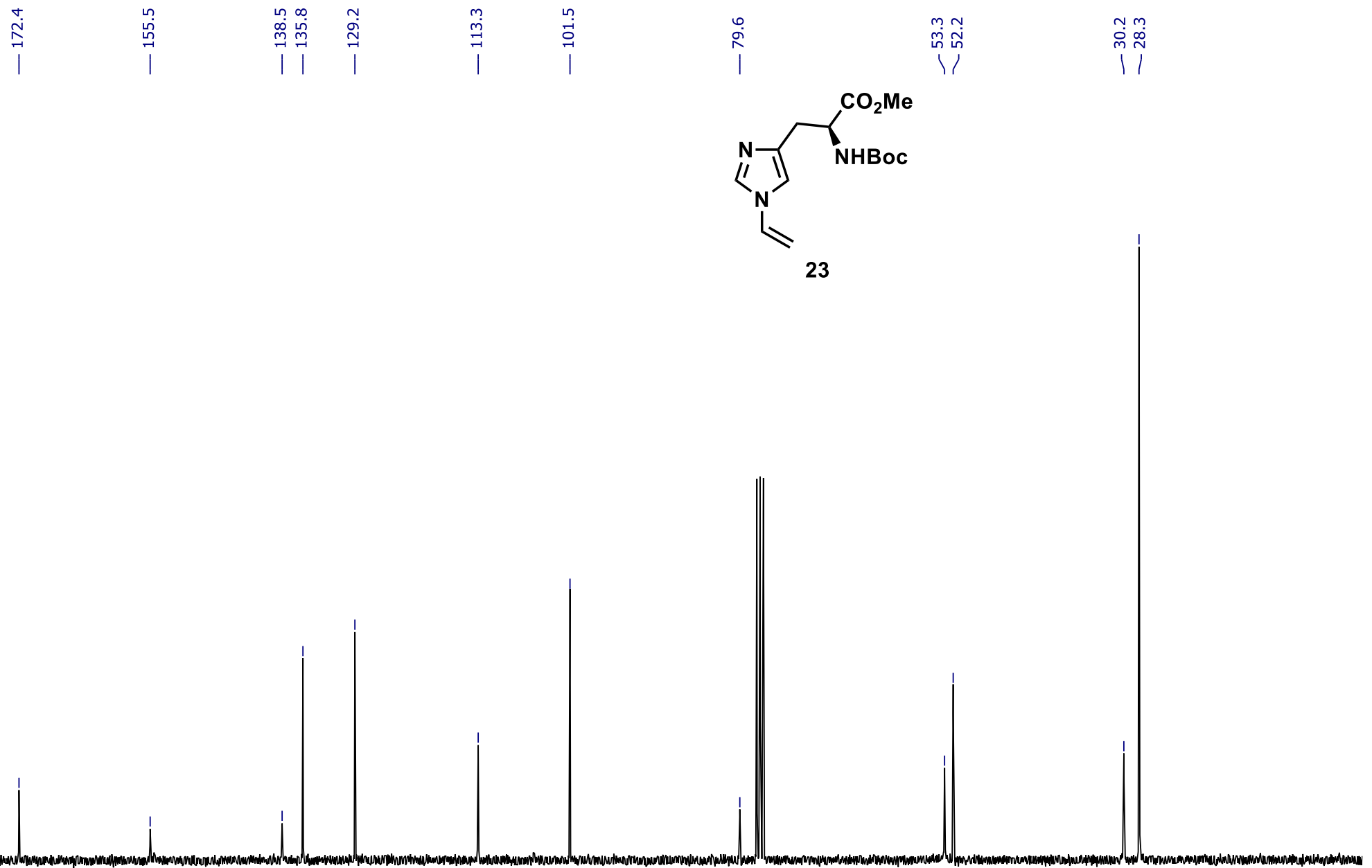

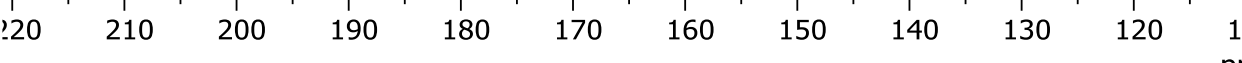

$\begin{array}{llllllllll}100 & 90 & 80 & 70 & 60 & 50 & 40 & 30 & 20 & 10\end{array}$


${ }^{1} \mathrm{H}$ NMR of $\mathrm{N}$-vinyl-metaxalone (24)

$\mathrm{CDCl}_{3}, 298 \mathrm{~K}$

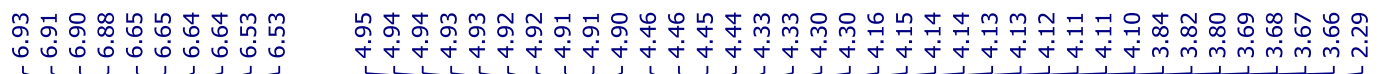

4 11

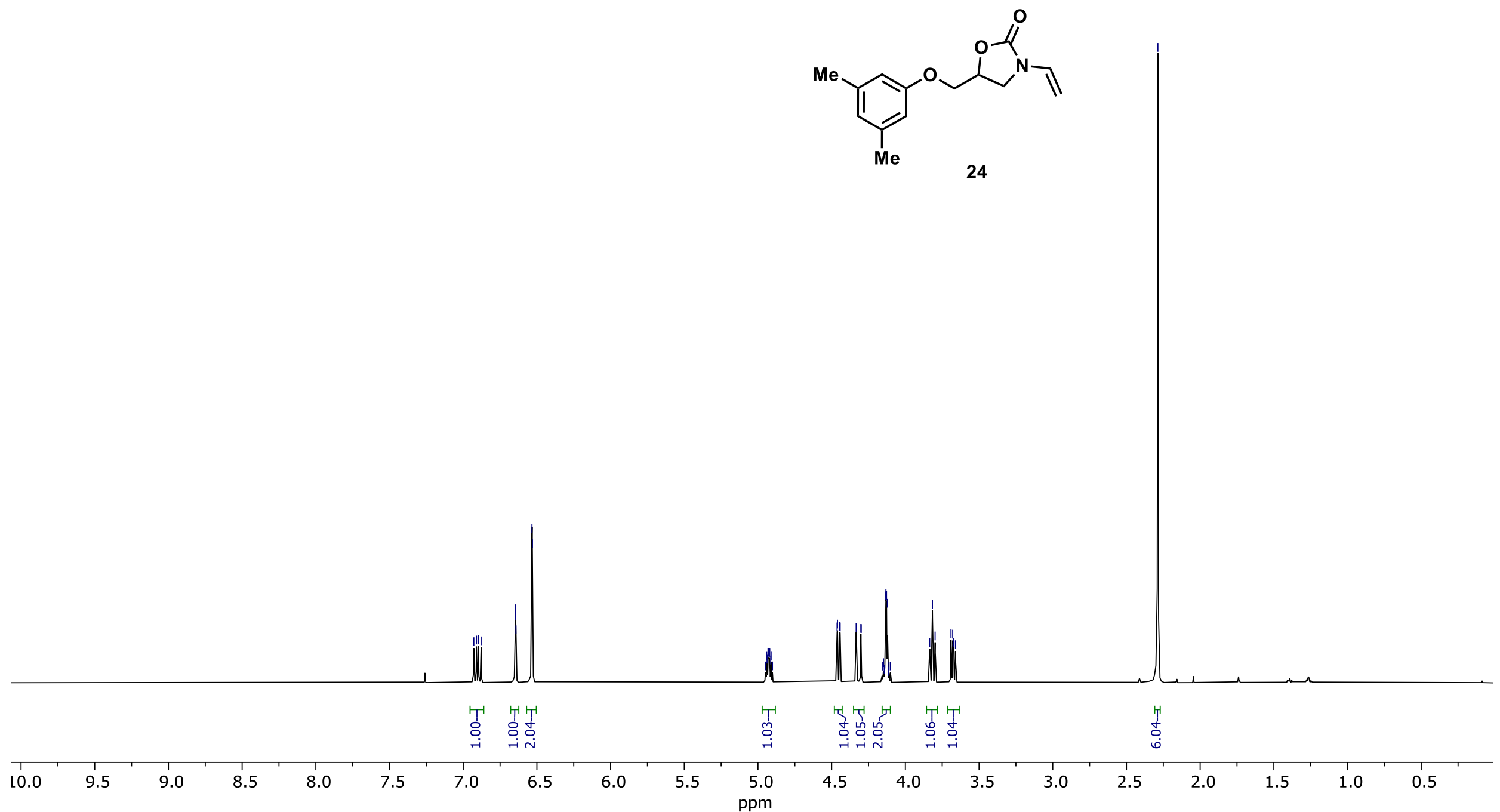


${ }^{13} \mathrm{C}$ NMR of $\mathrm{N}$-vinyl-metaxalone (24)

$\mathrm{CDCl}_{3}, 298 \mathrm{~K}$

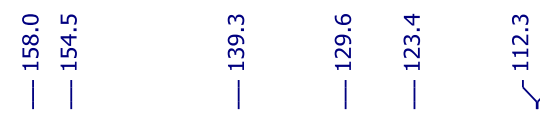
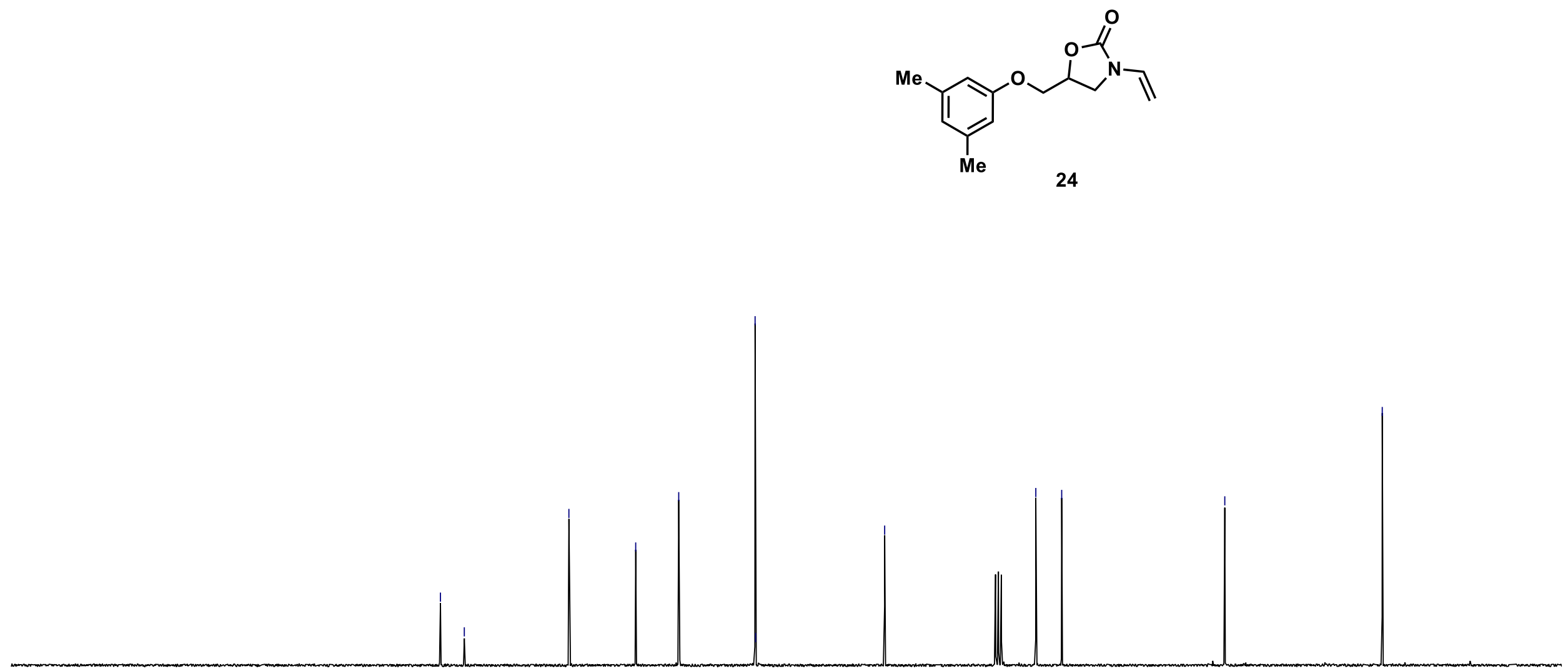

$\begin{array}{lllllll}20 & 210 & 200 & 190 & 180 & 170 & 160\end{array}$

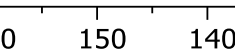

$130 \quad 120$ $110 \quad 100$ $90 \quad 80$ $\begin{array}{llll}70 & 60 & 50 & 40\end{array}$ $\begin{array}{llllll}1 & 1 & 1 & 1 & 1\end{array}$ 


\section{${ }^{1} \mathrm{H}$ NMR of $\mathrm{N}$-vinyl-N'-Boc-carvedilol (25)}

$\mathrm{CD}_{2} \mathrm{Cl}_{2}, 298 \mathrm{~K}$

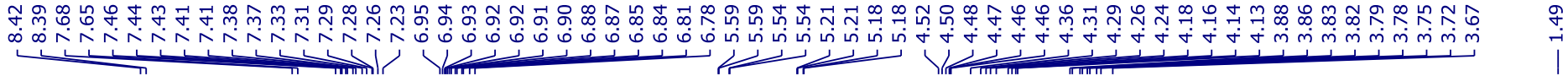

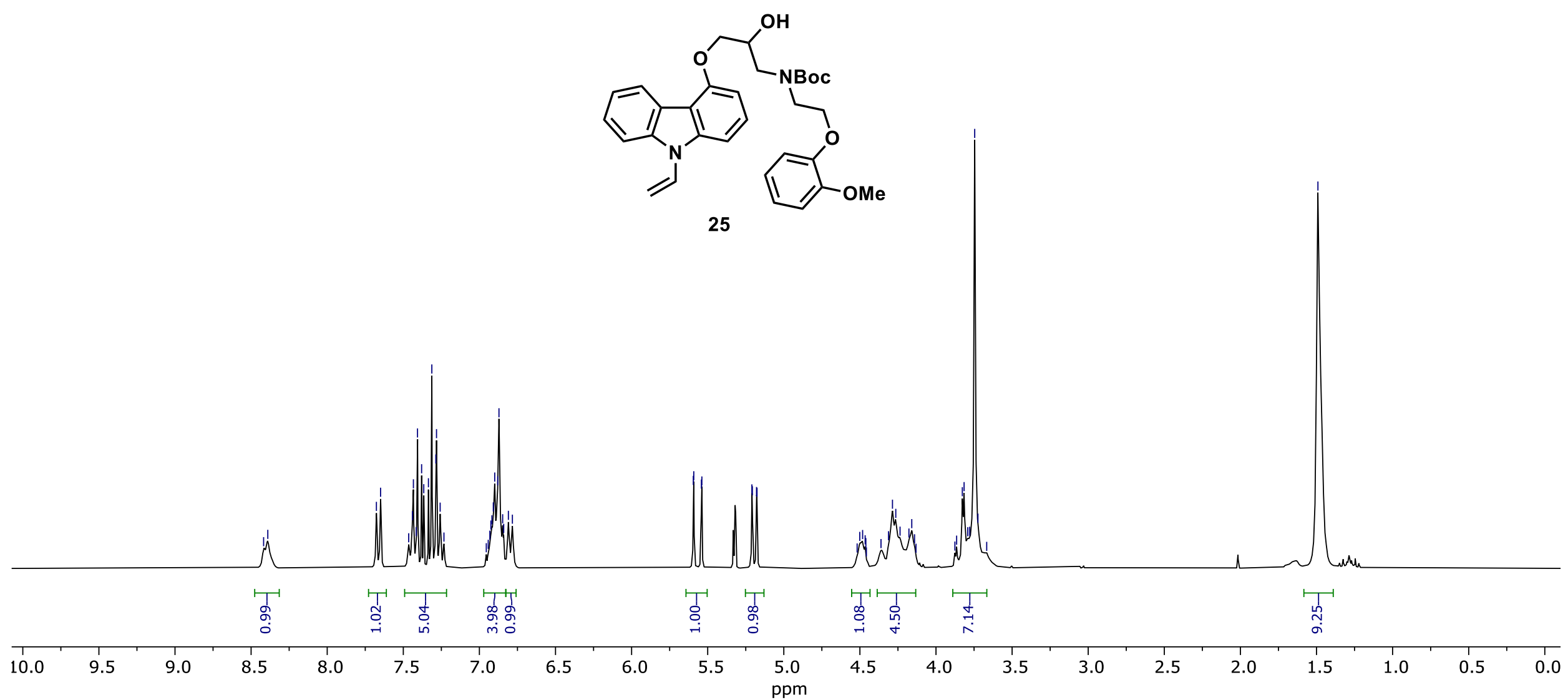


${ }^{13} \mathrm{C}$ NMR of $\mathrm{N}$-vinyl-N'-Boc-carvedilol (25)

$\mathrm{CD}_{2} \mathrm{Cl}_{2}, 298 \mathrm{~K}$
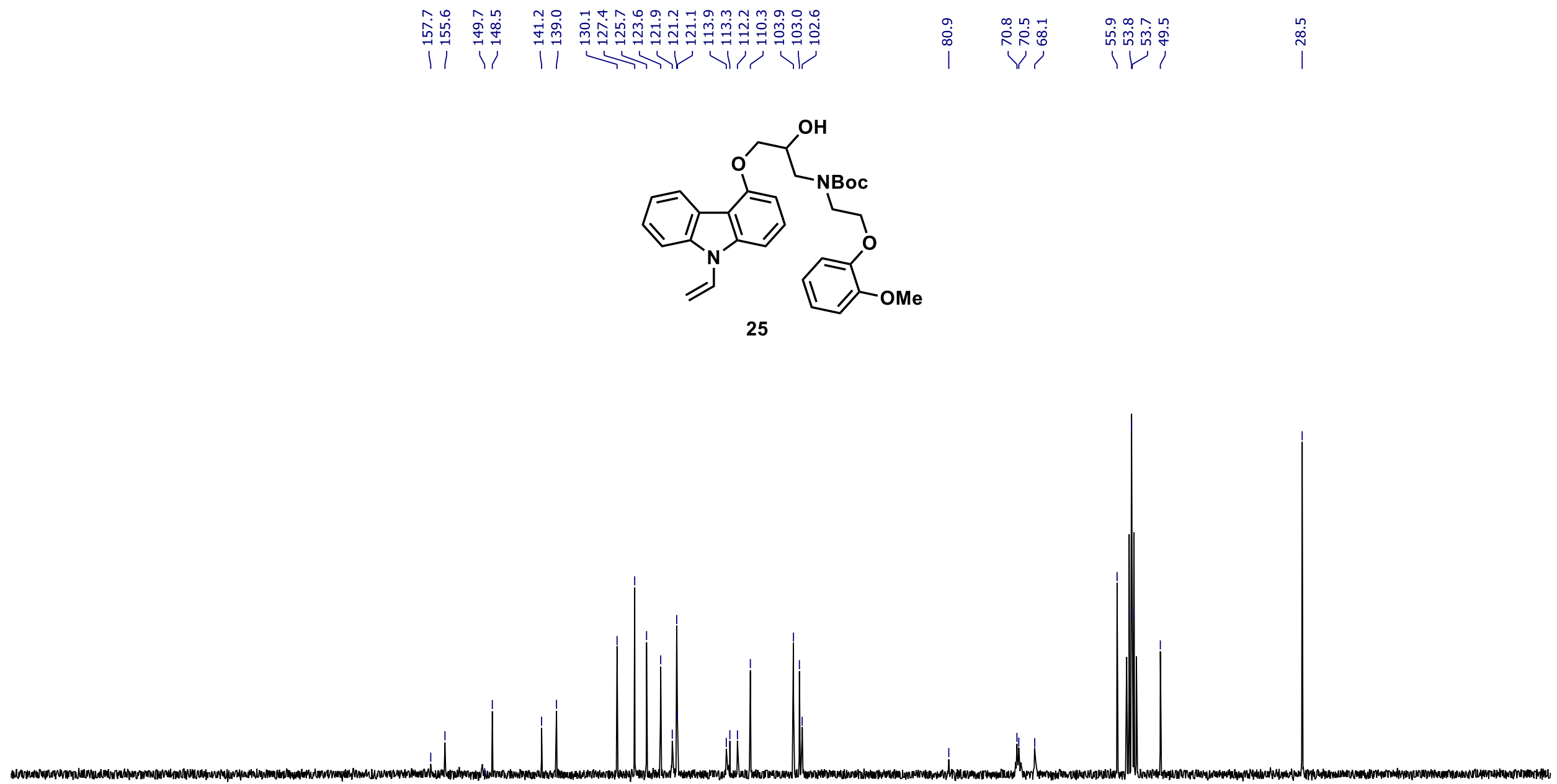


\section{${ }^{1} \mathrm{H}$ NMR of $\mathrm{N}$-vinyl-coumarin 7 (26)}

$\mathrm{CDCl}_{3}, 298 \mathrm{~K}$

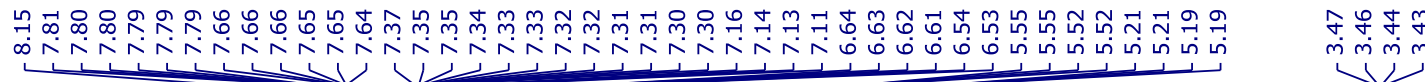

(1)<smiles>C=Cn1c(-c2cc3ccc(N(CC)CC)cc3oc2=O)nc2ccccc21</smiles>

26

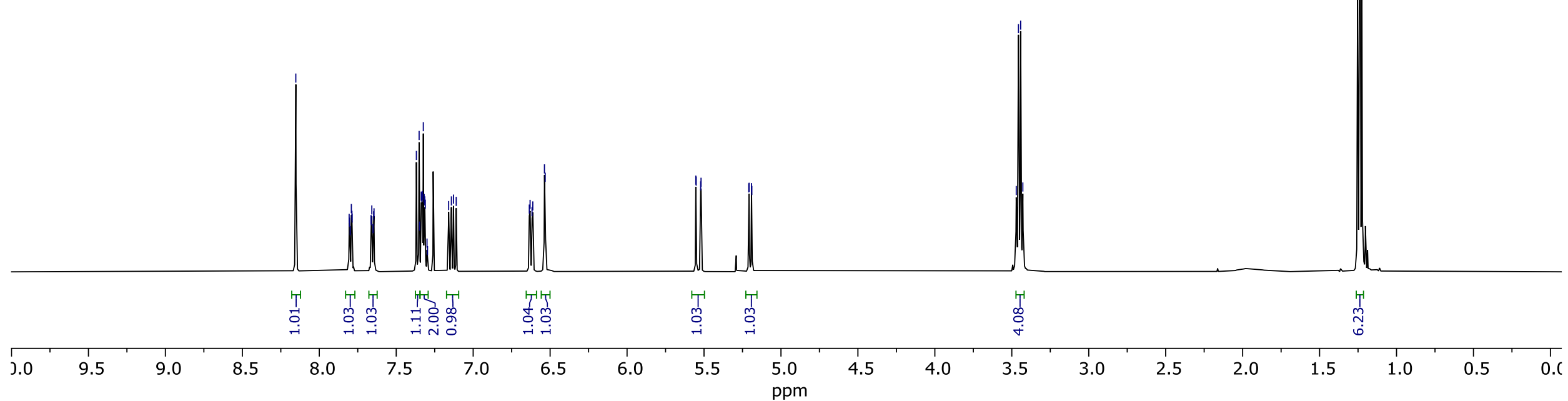


${ }^{13} \mathrm{C}$ NMR of $\mathrm{N}$-vinyl-coumarin 7 (26)

$\mathrm{CDCl}_{3}, 298 \mathrm{~K}$
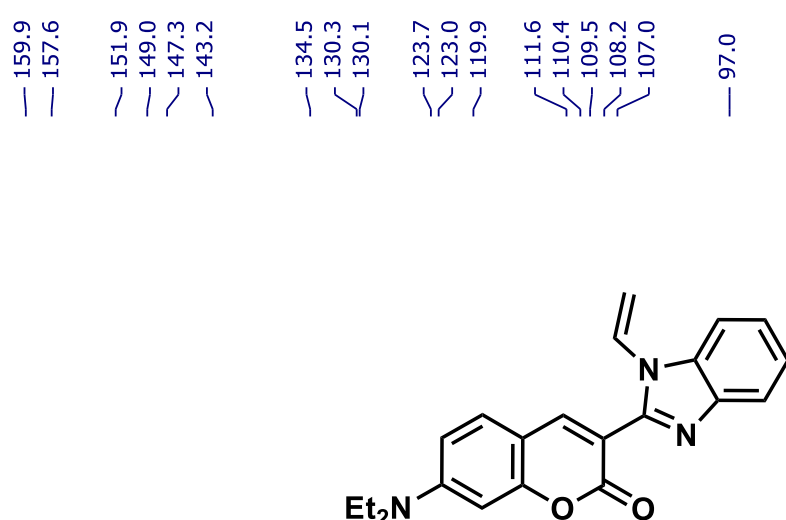

26

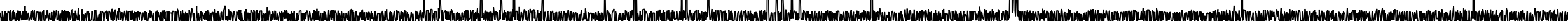




\section{${ }^{1} \mathrm{H}$ NMR of $\mathrm{N}$-vinyl-lansoprazole (27)}

$\mathrm{CD}_{2} \mathrm{Cl}_{2}, 298 \mathrm{~K}$

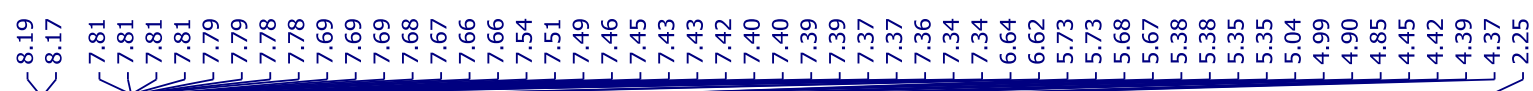
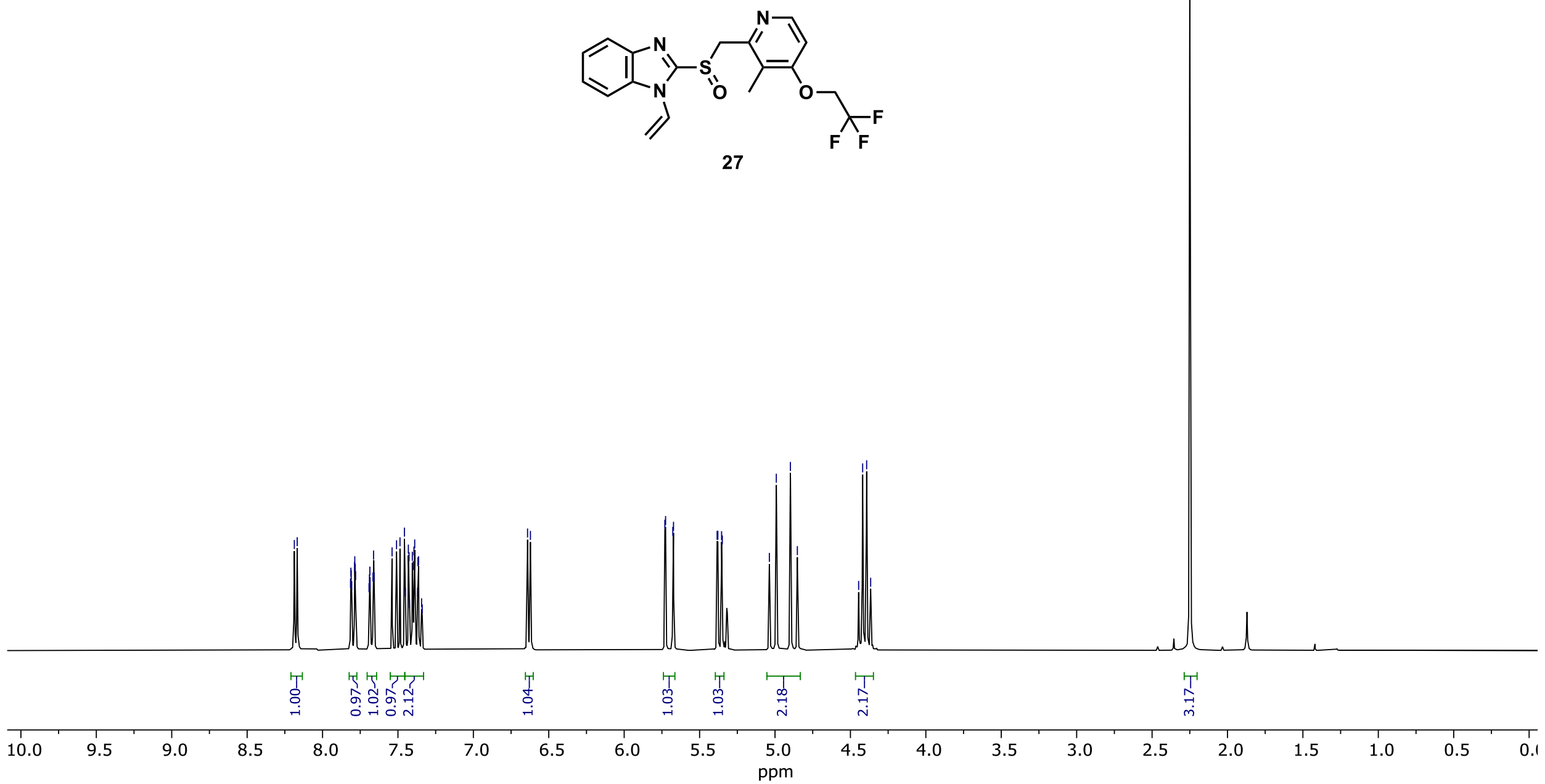
${ }^{13} \mathrm{C}$ NMR of $\mathrm{N}$-vinyl-lansoprazole (27)

$\mathrm{CD}_{2} \mathrm{Cl}_{2}, 298 \mathrm{~K}$

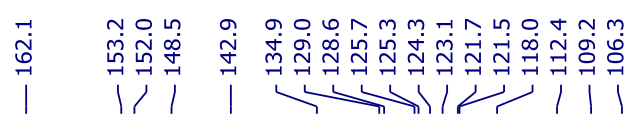

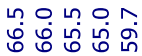

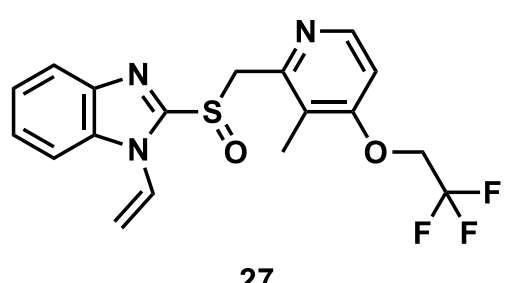

27

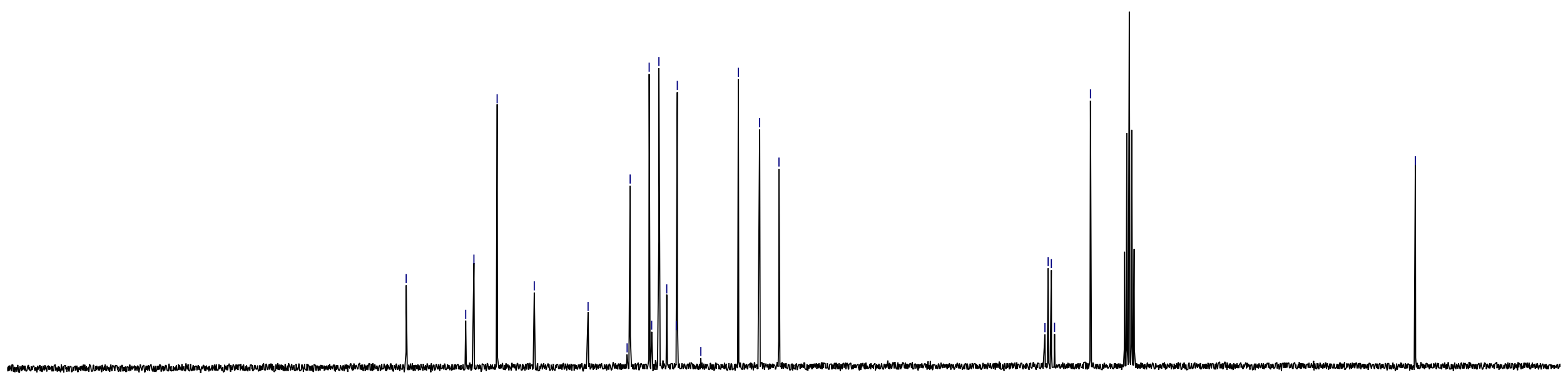

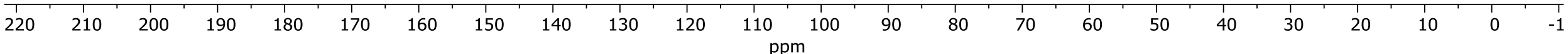


${ }^{19}$ F NMR of $\mathrm{N}$-vinyl-lansoprazole (27)

$\mathrm{CD}_{2} \mathrm{Cl}_{2}, 298 \mathrm{~K}$

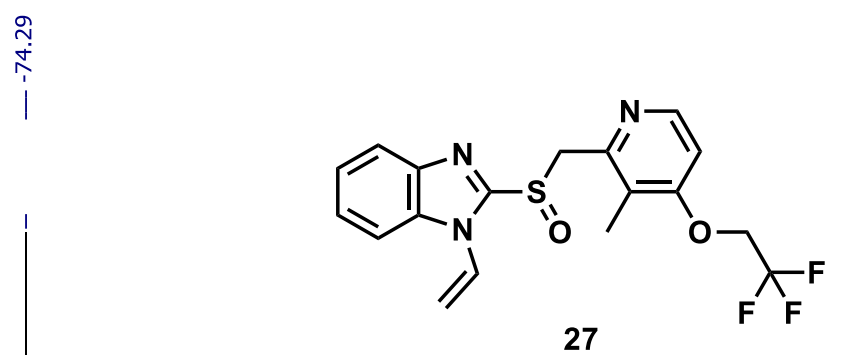

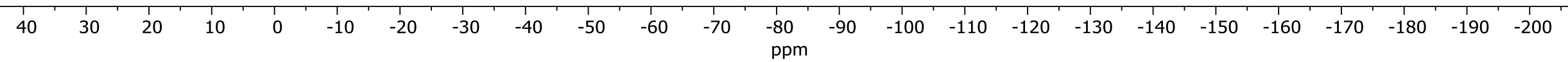


${ }^{1} \mathrm{H}$ NMR of 3-methyl-4-vinylbenzonitrile (29)

$\mathrm{CDCl}_{3}, 298 \mathrm{~K}$

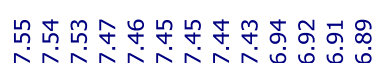

年

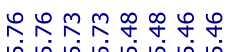

年

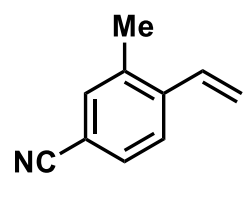

29

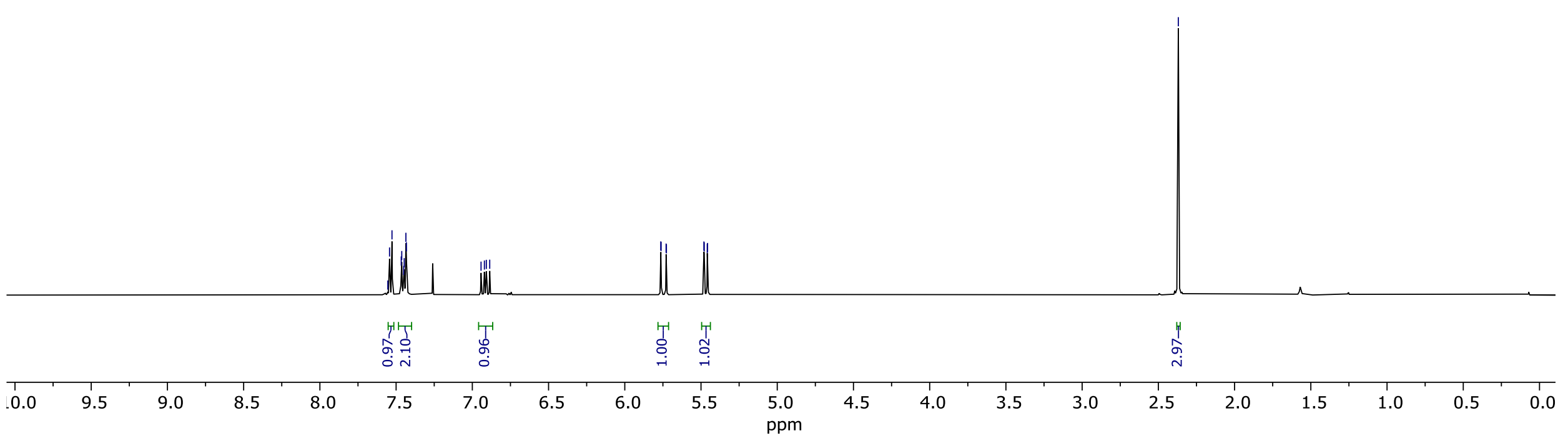


${ }^{13} \mathrm{C}$ NMR of 3-methyl-4-vinylbenzonitrile (29)

$\mathrm{CDCl}_{3}, 298 \mathrm{~K}$
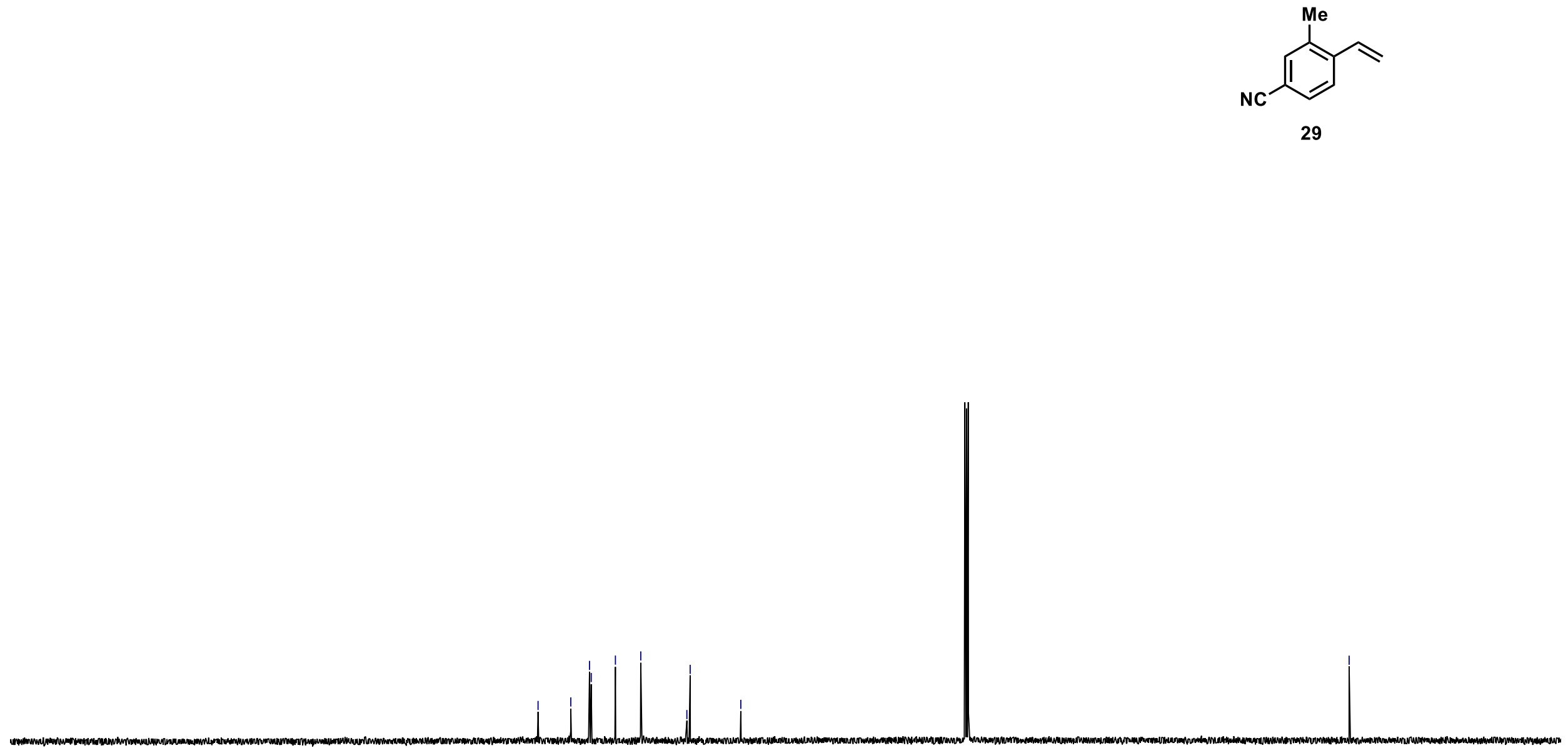
${ }^{1} \mathrm{H}$ NMR of 1-chloro-4-methoxy-2-vinylbenzene (30)

$\mathrm{CDCl}_{3}, 298 \mathrm{~K}$

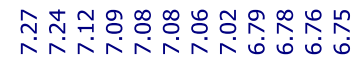
선

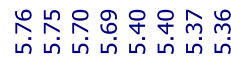

$\underbrace{2}_{4}$

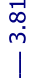

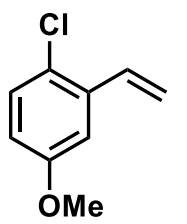

30

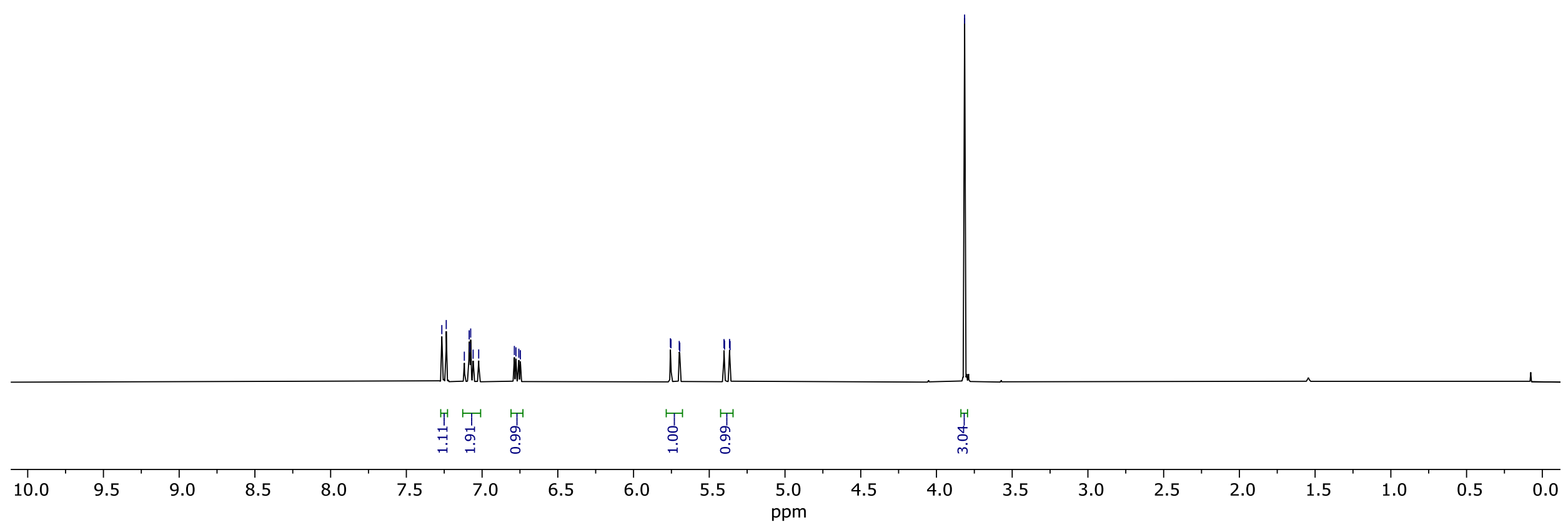


${ }^{13} \mathrm{C}$ NMR of 1-chloro-4-methoxy-2-vinylbenzene (30)

$\mathrm{CDCl}_{3}, 298 \mathrm{~K}$

$$
\text { 㸃 }
$$

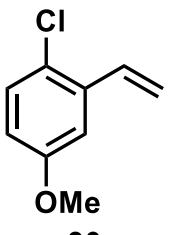

30

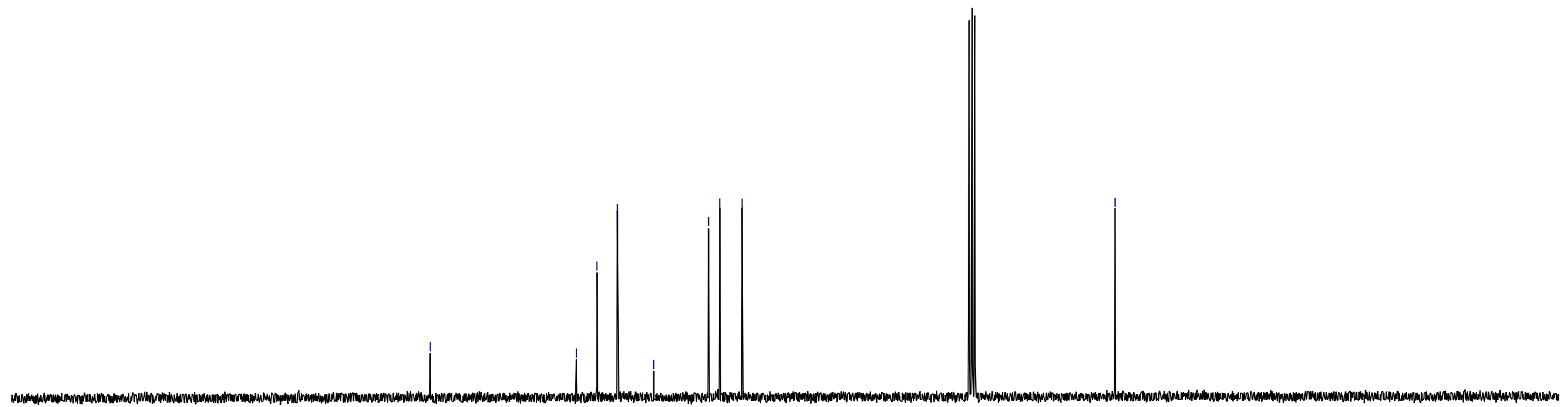


${ }^{1} \mathrm{H}$ NMR of 1-chloro-4-vinylbenzene (31)

$\mathrm{CDCl}_{3}, 298 \mathrm{~K}$

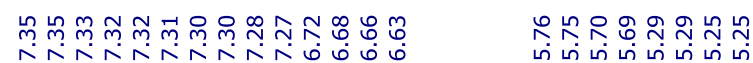

प पै

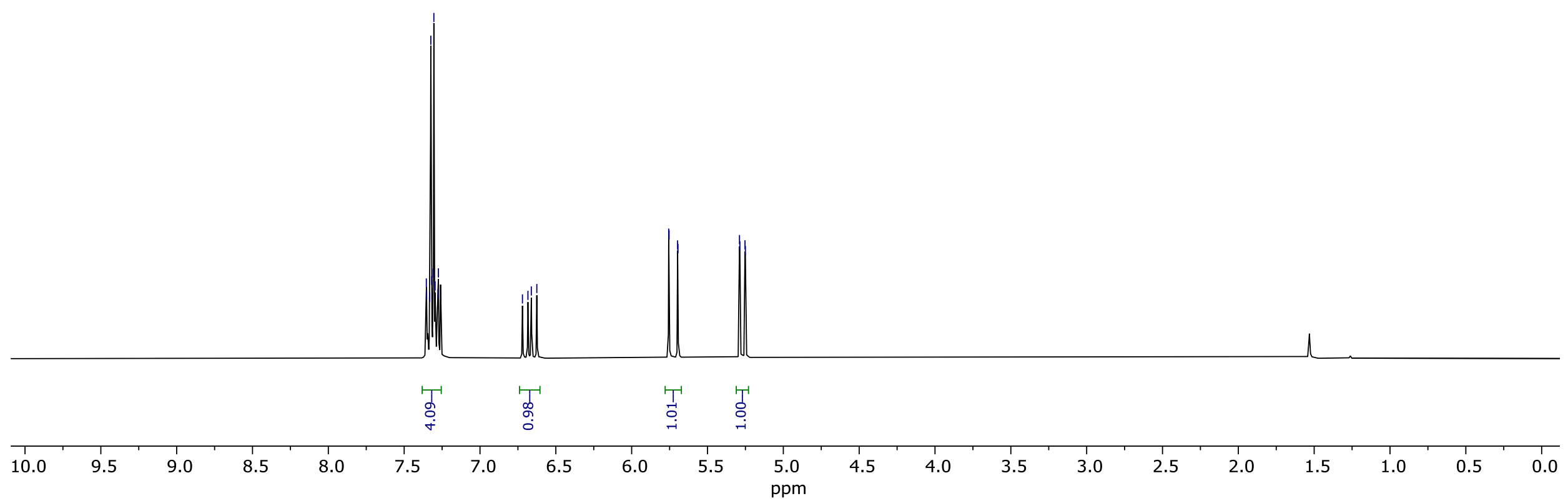


${ }^{13} \mathrm{C} \mathrm{NMR}$ of 1-chloro-4-vinylbenzene (31)
$\mathrm{CDCl}_{3}, 298 \mathrm{~K}$

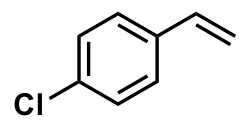

31

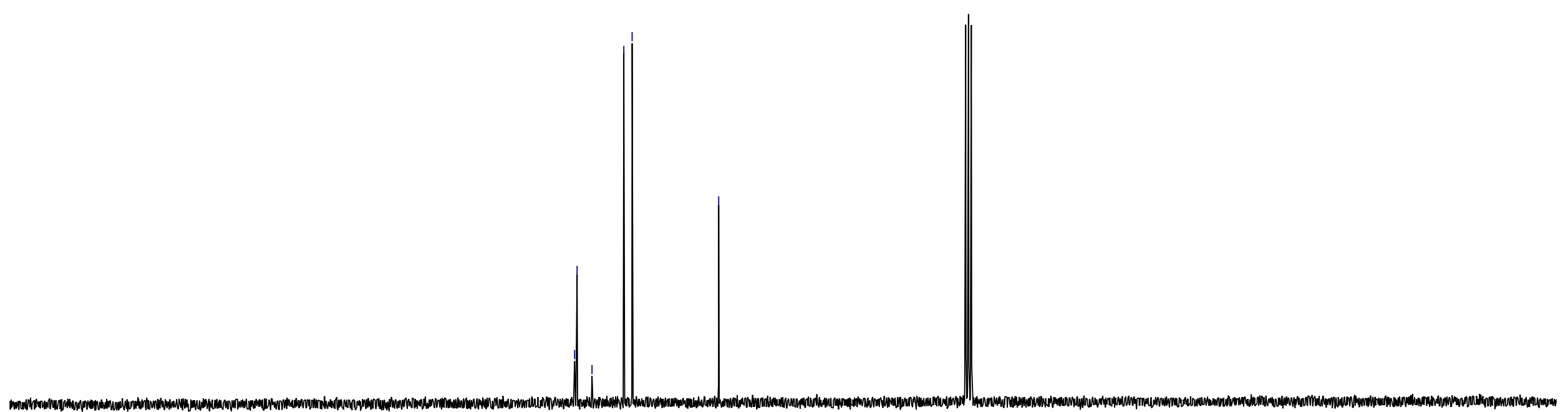

$201210 \quad 200$

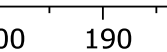

$180 \quad 170$

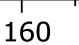

150
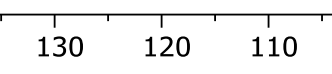

ppm 
${ }^{1} \mathrm{H}$ NMR of 1 -chloro-4-(vinyl- $\left.d_{3}\right)$ benzene $\left(31-d_{3}\right)$

$\mathrm{CD}_{2} \mathrm{Cl}_{2}, 298 \mathrm{~K}$

\section{ํㅐㅆำ}

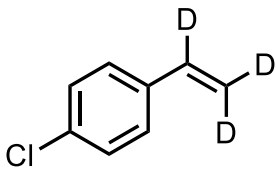

$31-d_{3}$

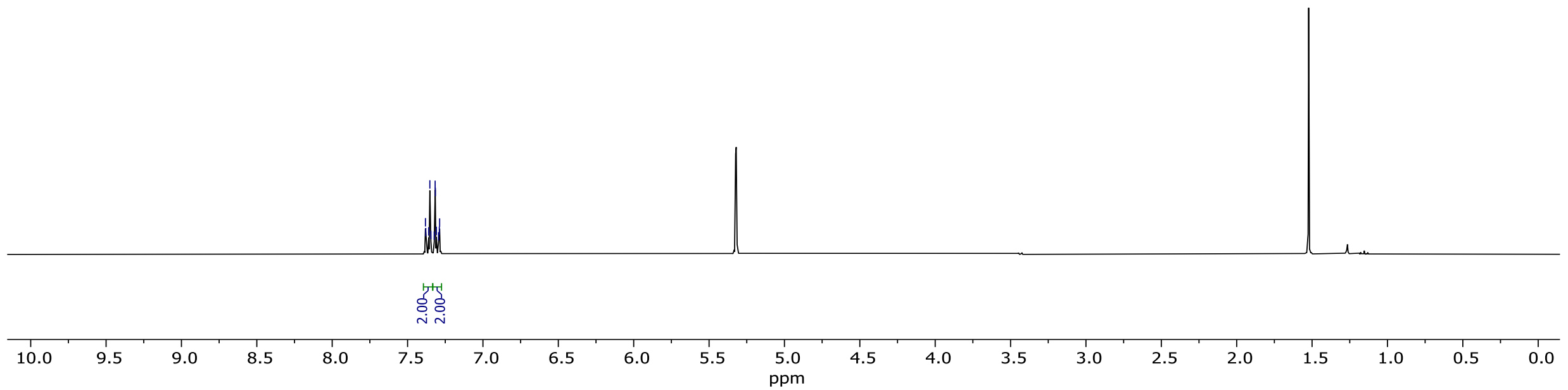




\section{${ }^{2} \mathrm{H}$ NMR of 1-chloro-4-(vinyl- $\left.d_{3}\right)$ benzene $\left(31-d_{3}\right)$}

$\mathrm{CD}_{2} \mathrm{Cl}_{2}, 298 \mathrm{~K}$

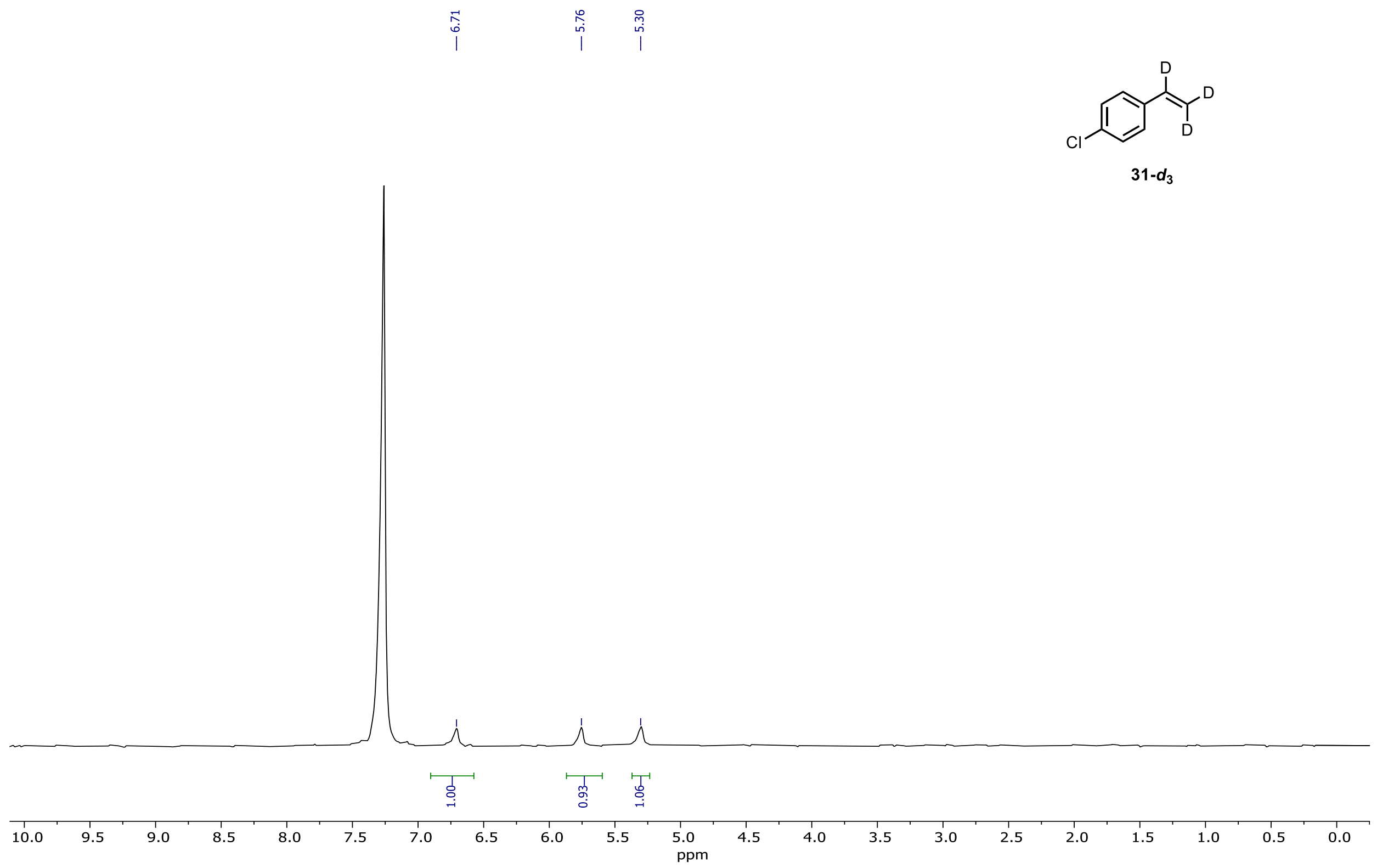


${ }^{13} \mathrm{C}$ NMR of 1 -chloro-4-(vinyl- $\left.d_{3}\right)$ benzene $\left(31-d_{3}\right)$

$\mathrm{CD}_{2} \mathrm{Cl}_{2}, 243 \mathrm{~K}$

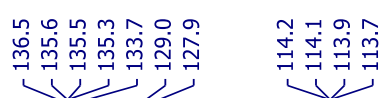
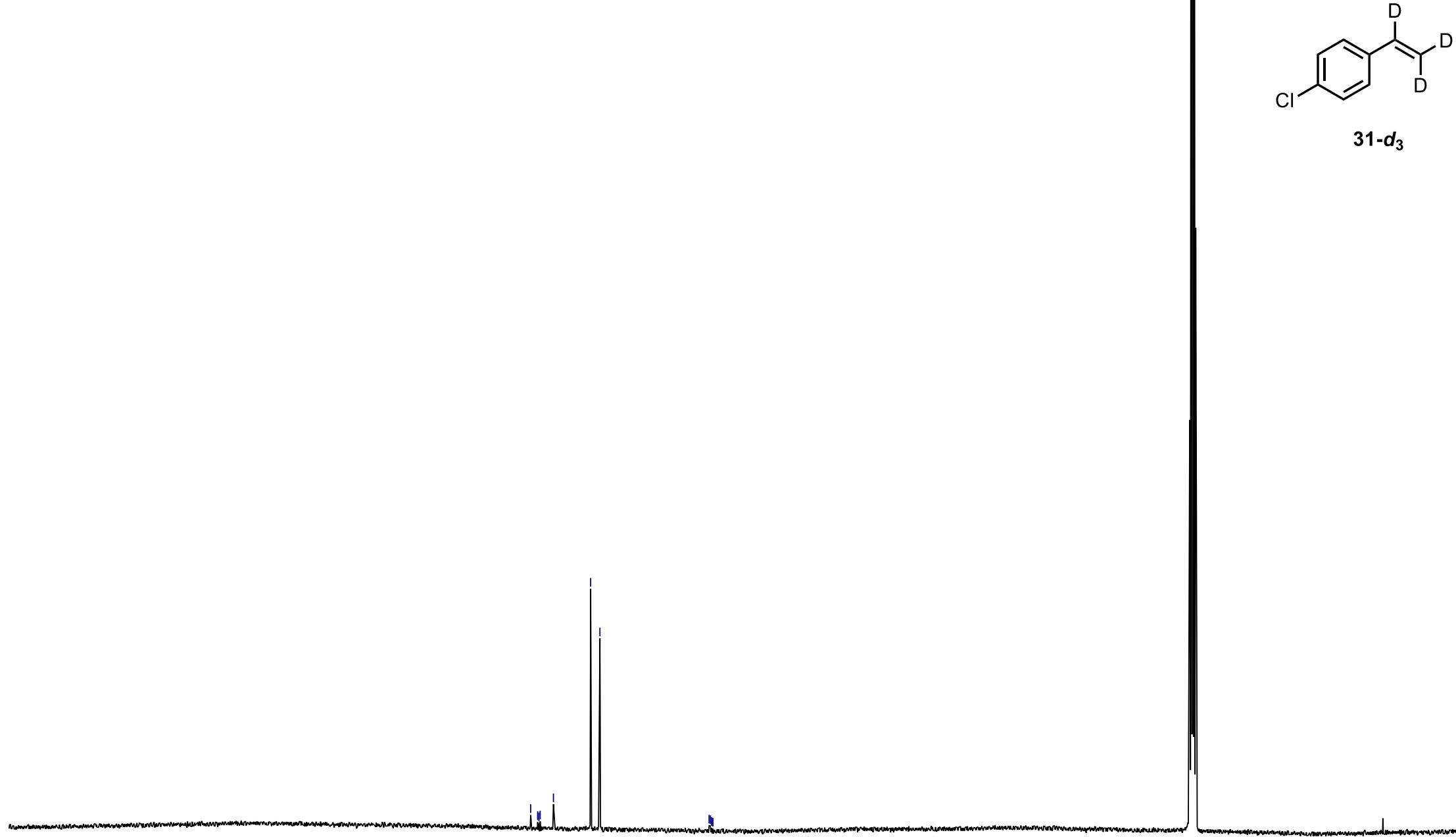
${ }^{1} \mathrm{H}$ NMR of 1-ethoxy-2-vinylbenzene (32)

$\mathrm{CDCl}_{3}, 298 \mathrm{~K}$

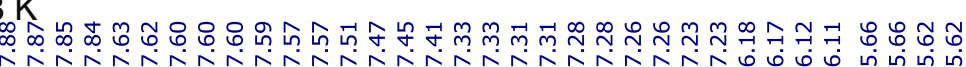

$\longrightarrow$

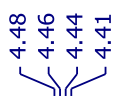

$\stackrel{\infty}{\infty} \infty \infty_{\infty}^{-\infty}$

ij

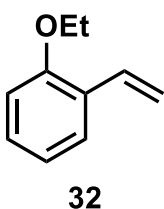

32

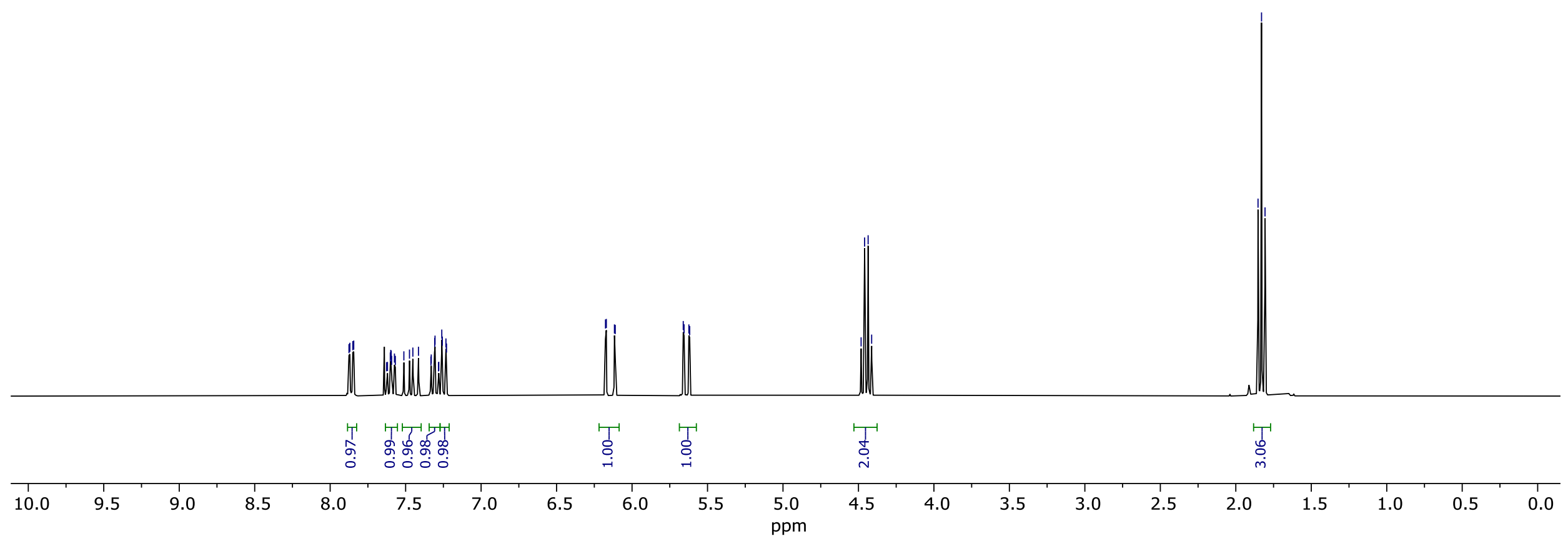


${ }^{13} \mathrm{C}$ NMR of 1-ethoxy-2-vinylbenzene (32)

$\mathrm{CDCl}_{3}, 298 \mathrm{~K}$
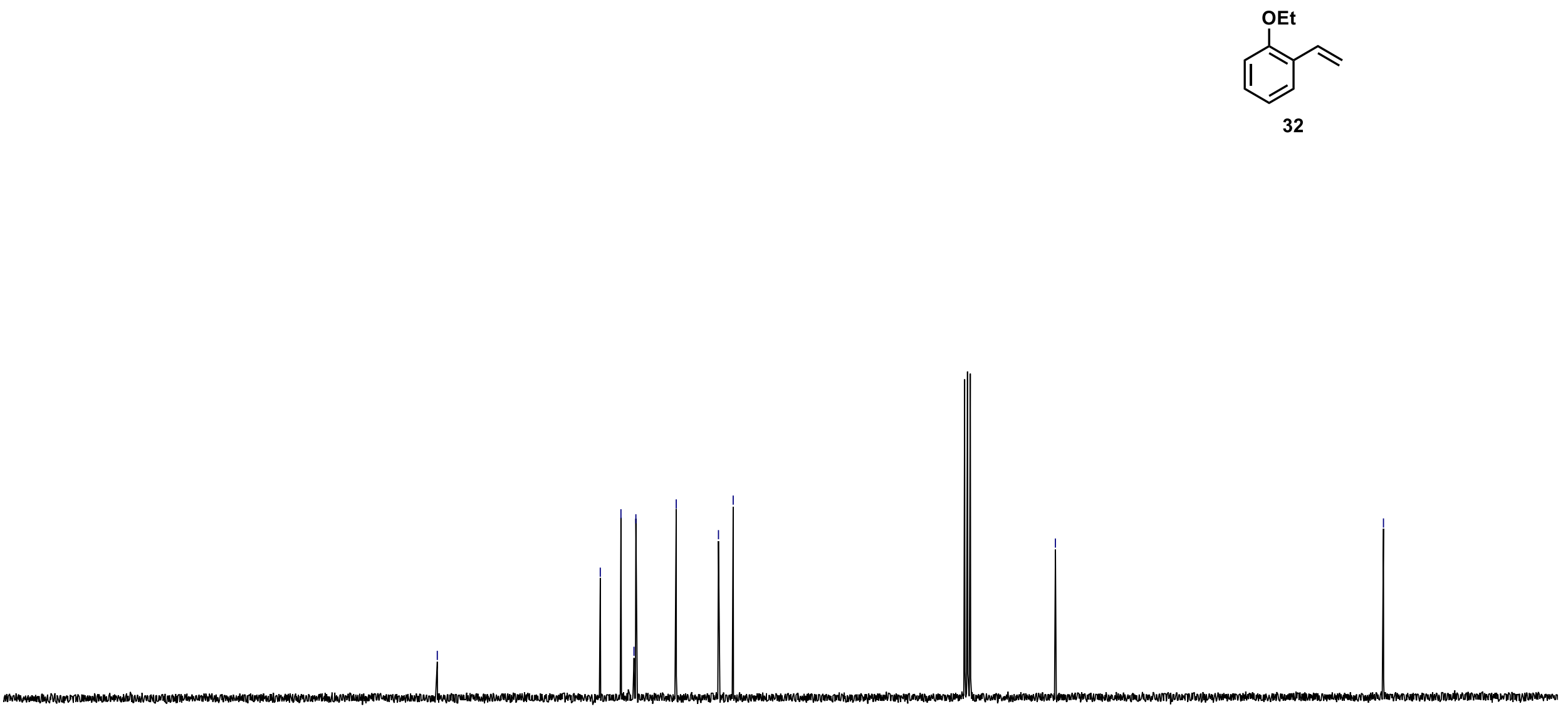

$220 \quad 210 \quad 200 \quad 190-180$ 
${ }^{1} \mathrm{H}$ NMR of 1-bromo-4-vinylbenzene (33)

$\mathrm{CDCl}_{3}, 298 \mathrm{~K}$

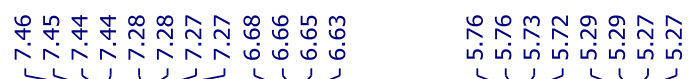

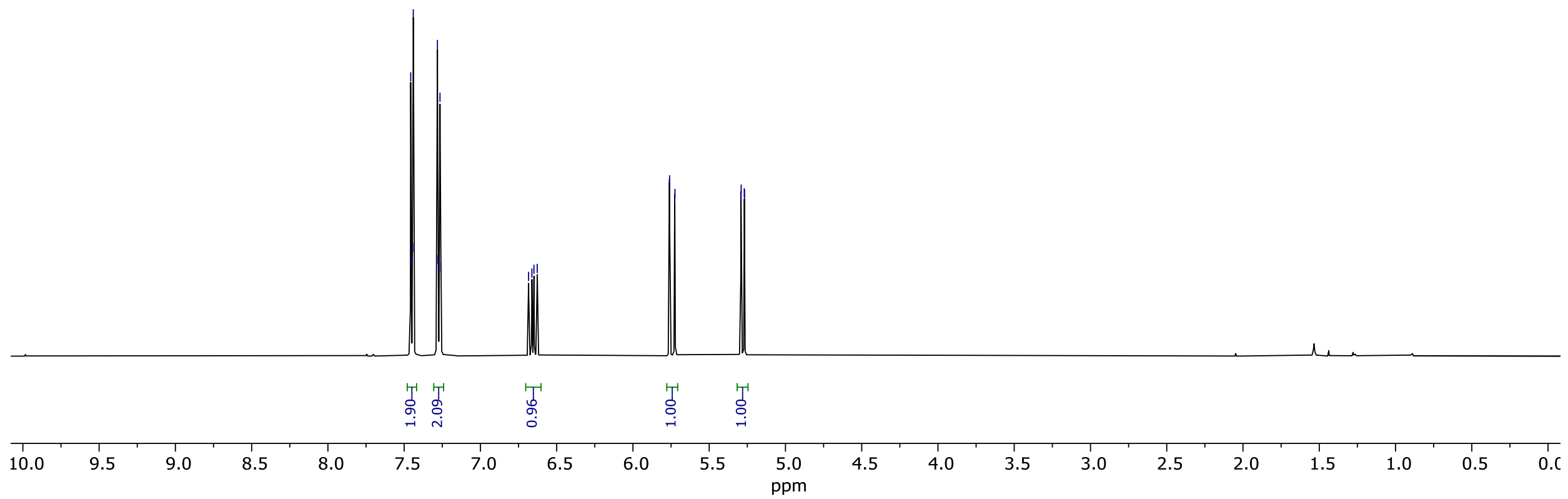


${ }^{13} \mathrm{C}$ NMR of 1-bromo-4-vinylbenzene (33)

$\mathrm{CDCl}_{3}, 298 \mathrm{~K}$

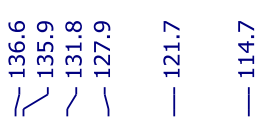
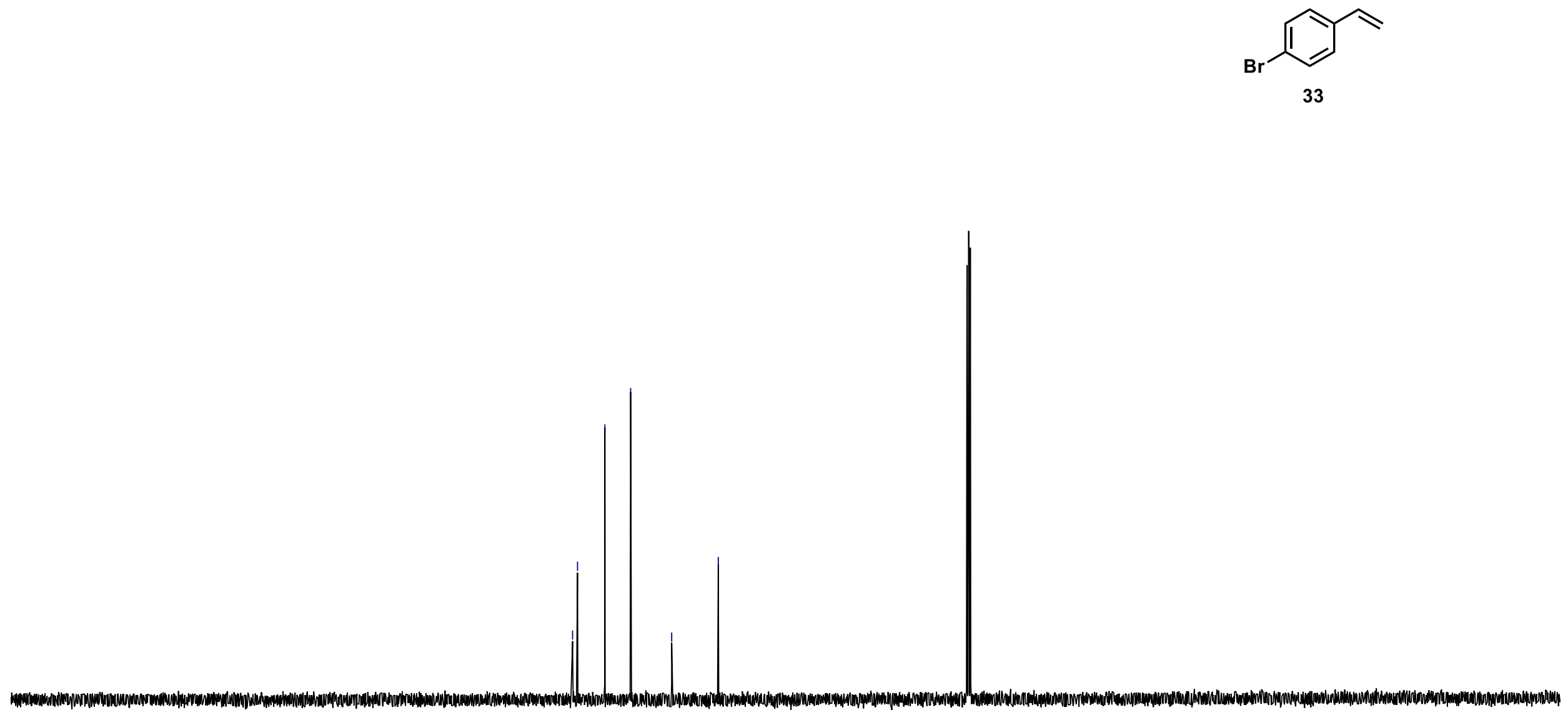

$\begin{array}{llllllllllllllllllllllllllllll}120 & 210 & 200 & 190 & 180 & 170 & 160 & 150 & 140 & 130 & 120 & 110 & 100 & 90 & 80 & 70 & 60 & 50 & 40 & 30 & 20 & 10 & 0 & -11\end{array}$


${ }^{1} \mathrm{H}$ NMR of 4-vinylbenzaldehyde (34)

$\mathrm{CDCl}_{3}, 298 \mathrm{~K}$

के

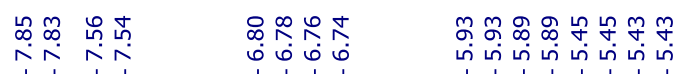

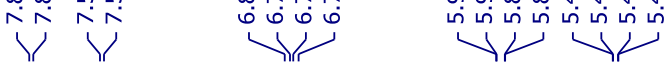
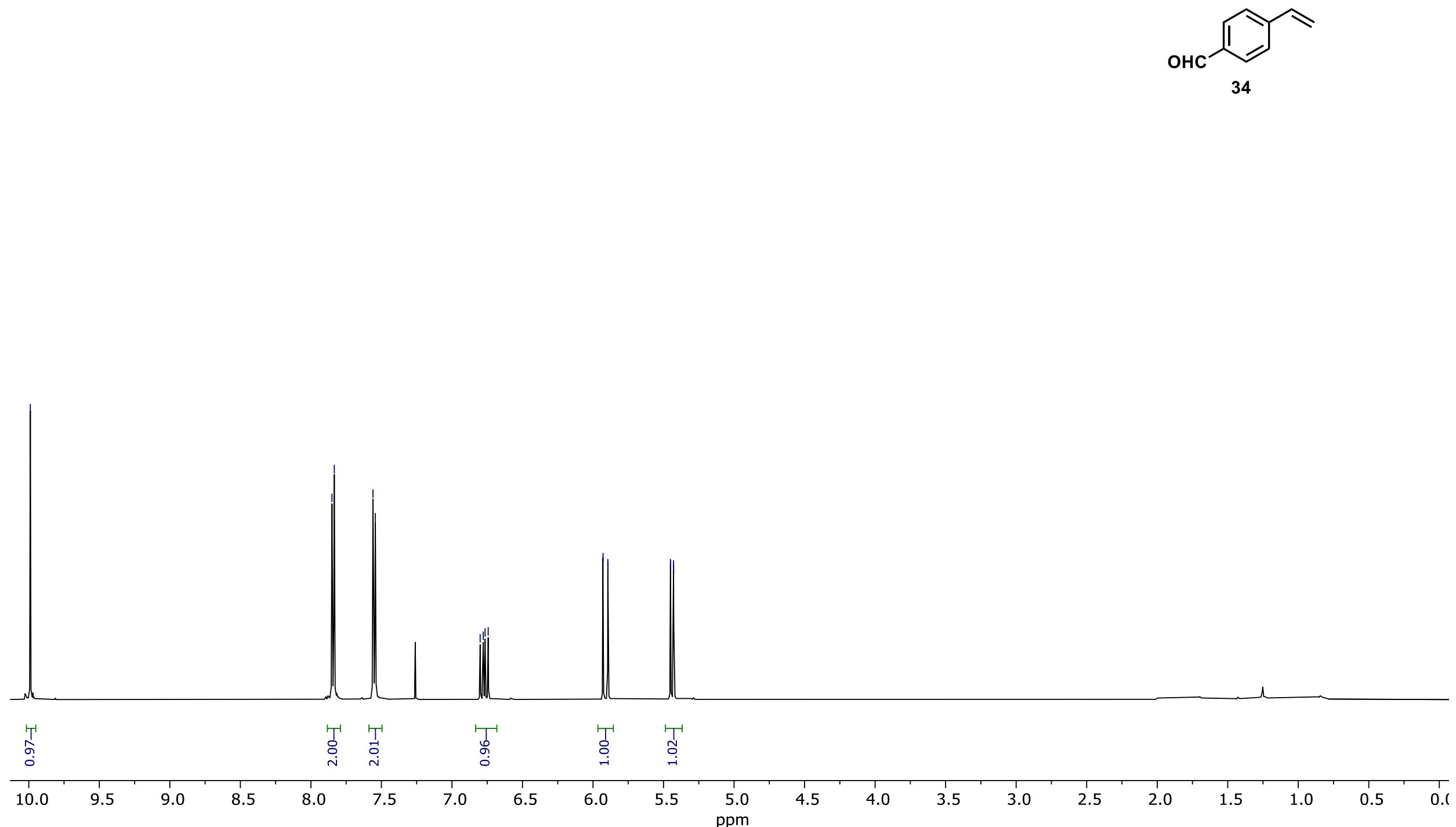
${ }^{13} \mathrm{C}$ NMR of 4-vinylbenzaldehyde (34)

$\mathrm{CDCl}_{3}, 298 \mathrm{~K} \stackrel{\text { जे }}{\vec{\sigma}}$

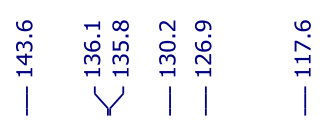
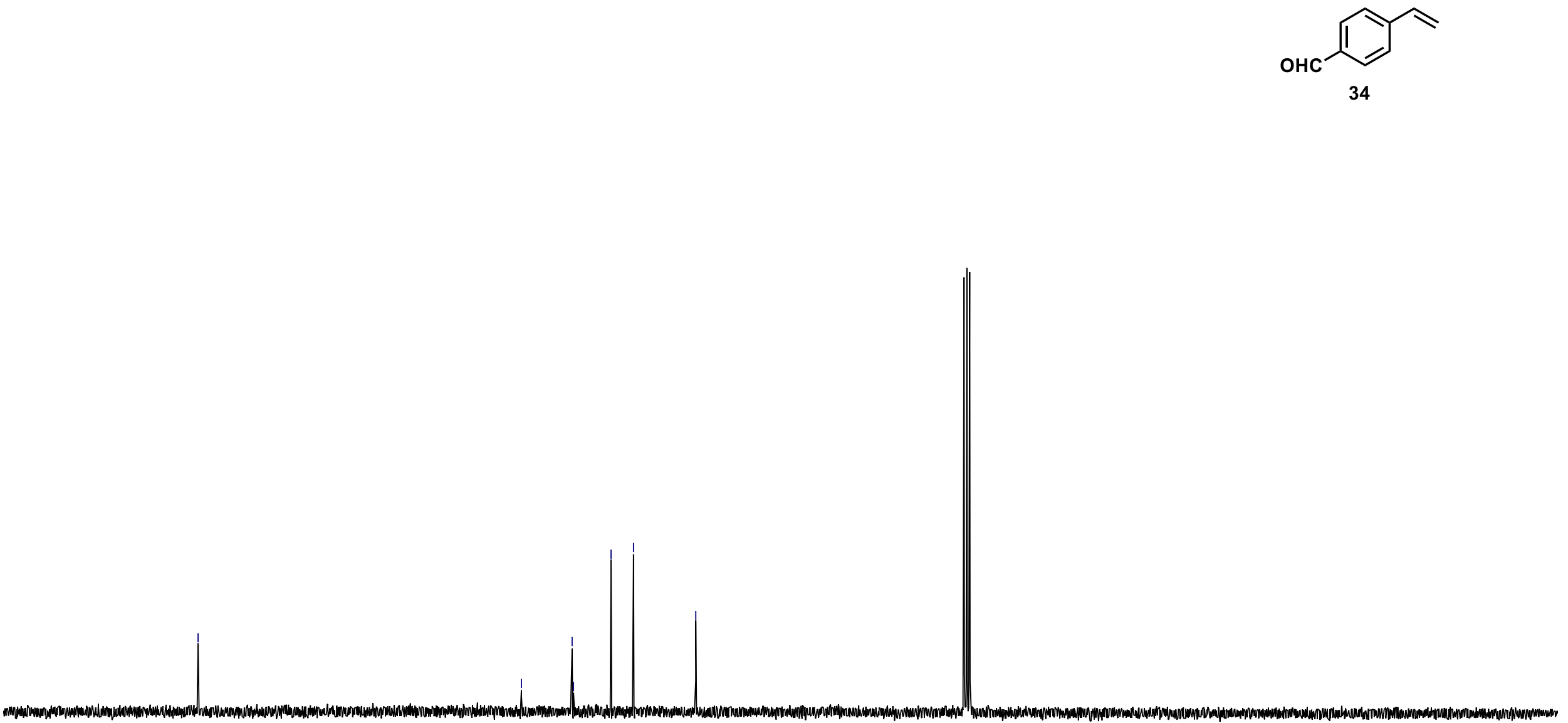

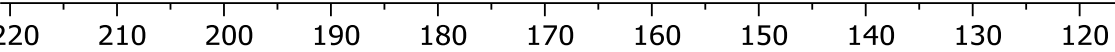


${ }^{1} \mathrm{H}$ NMR of 2-vinylbenzo[b]thiophene (35)

$\mathrm{CDCl}_{3}, 298 \mathrm{~K}$

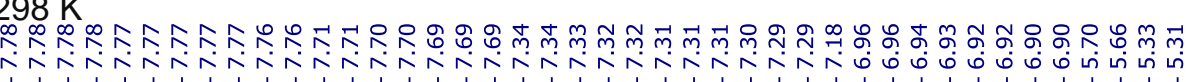

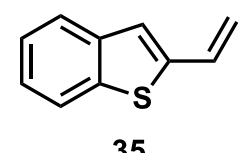

35

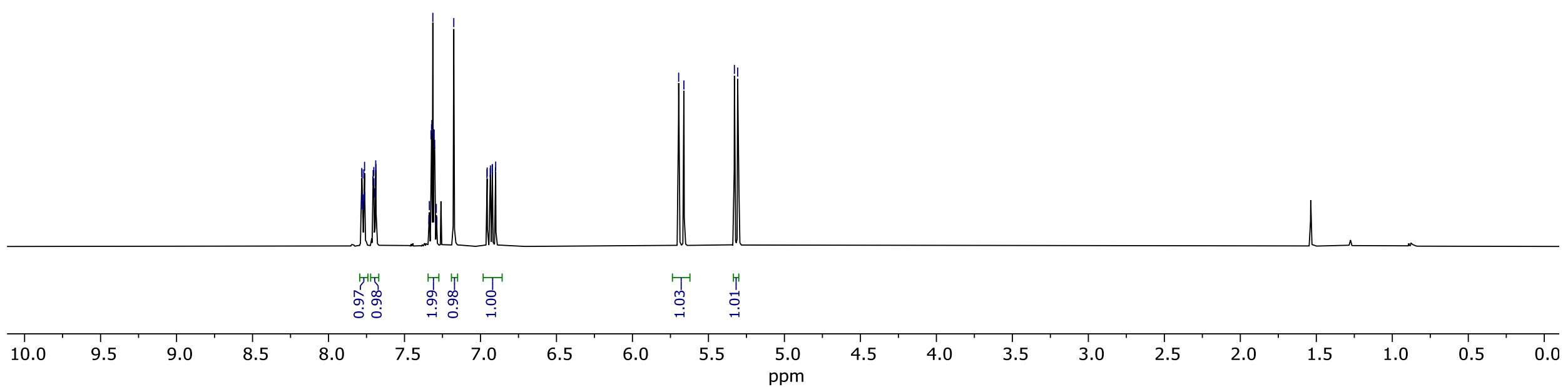


${ }^{13} \mathrm{C}$ NMR of 2-vinylbenzo[b]thiophene (35)

$\mathrm{CDCl}_{3}, 298 \mathrm{~K}$

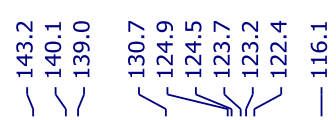
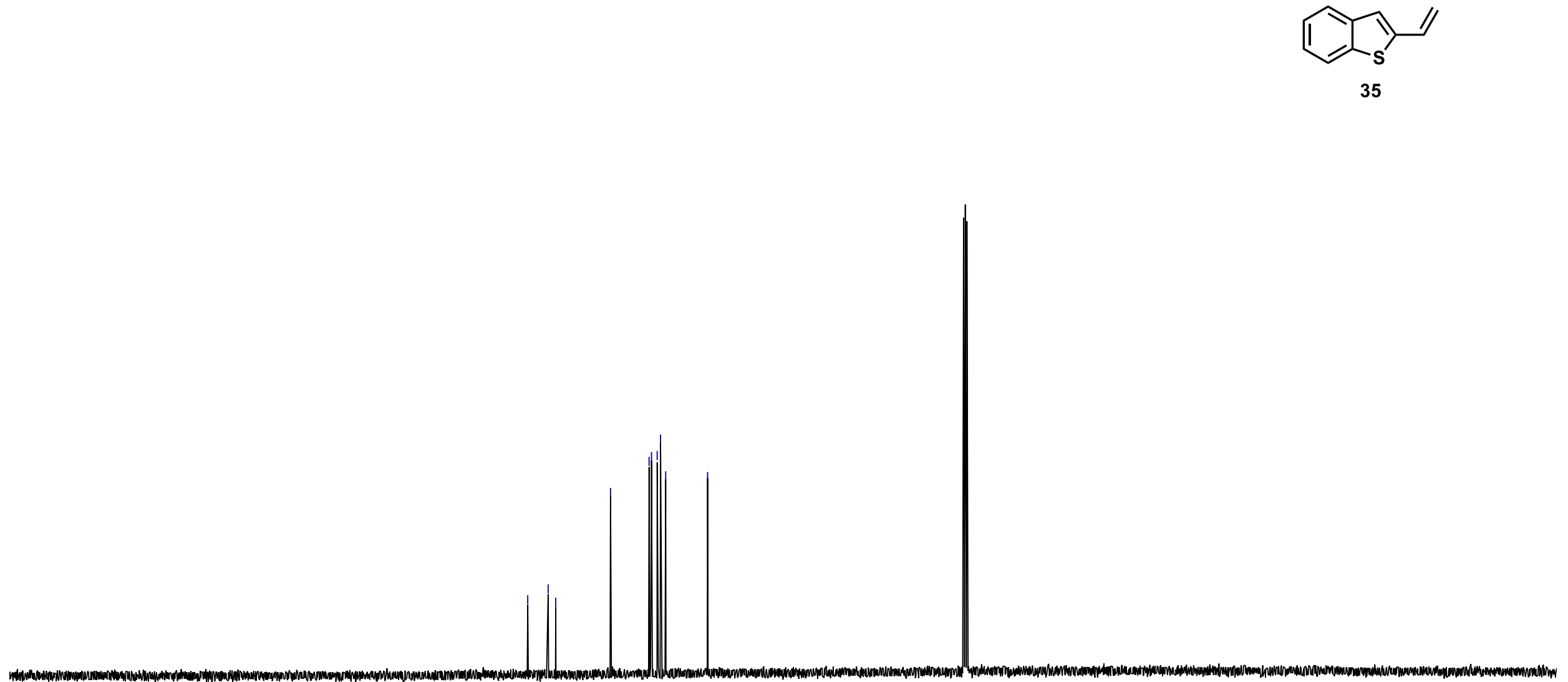

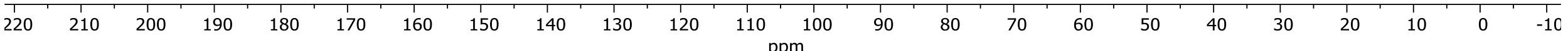


${ }^{1} \mathrm{H}$ NMR of 1-methyl-3-(trifluoromethyl)-5-vinyl-1 $H$-pyrazole (36)

$\mathrm{CDCl}_{3}, 298 \mathrm{~K}$

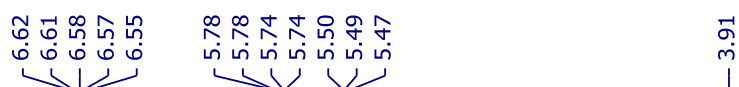

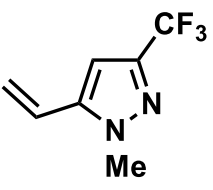

36

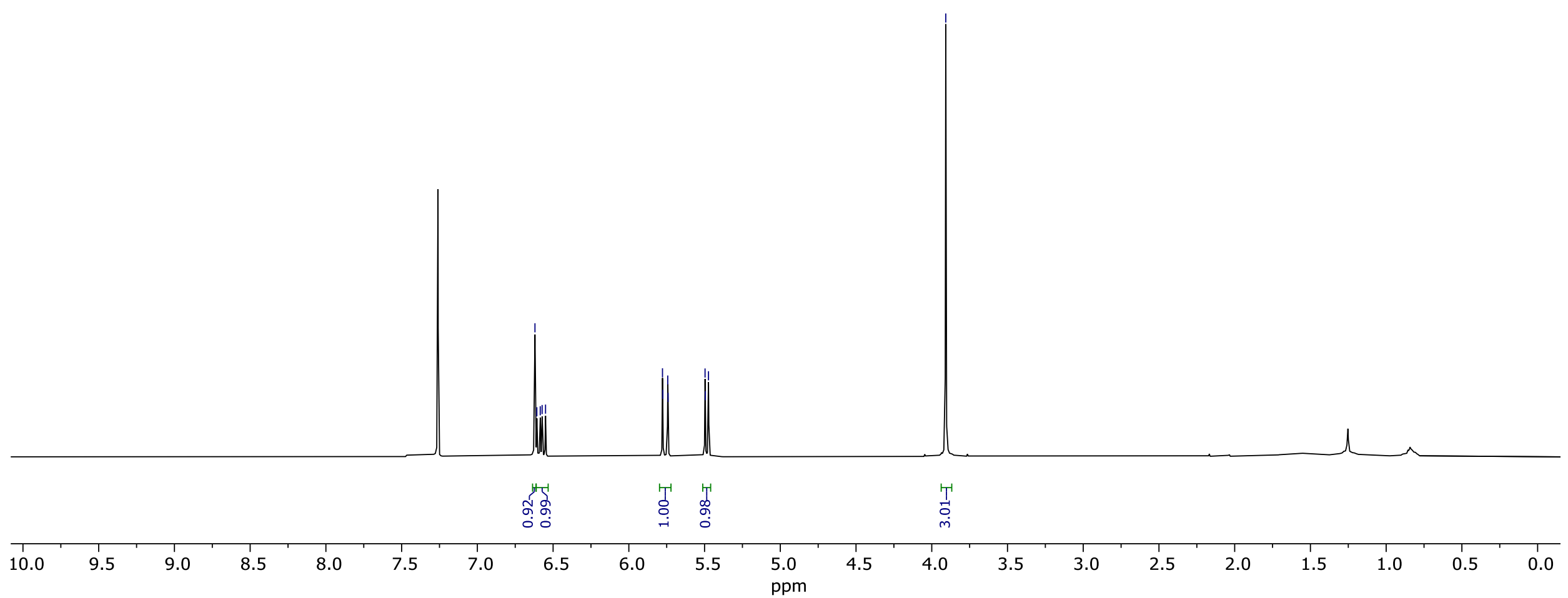


${ }^{13} \mathrm{C}$ NMR of 1-methyl-3-(trifluoromethyl)-5-vinyl-1 H-pyrazole (36)

$\mathrm{CDCl}_{3}, 298 \mathrm{~K}$

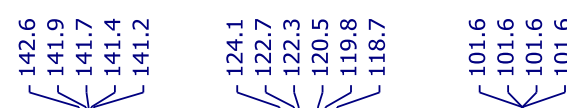

$\stackrel{+}{\dot{m}}$

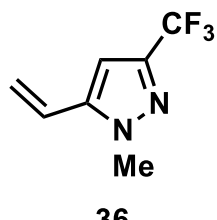

36

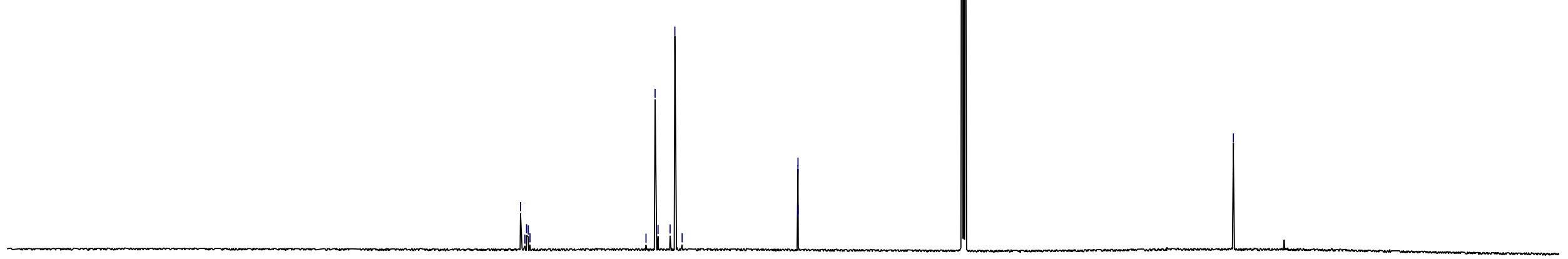


${ }^{19} \mathrm{~F}$ NMR of 1-methyl-3-(trifluoromethyl)-5-vinyl-1 H-pyrazole (36)

$\mathrm{CDCl}_{3}, 298 \mathrm{~K}$

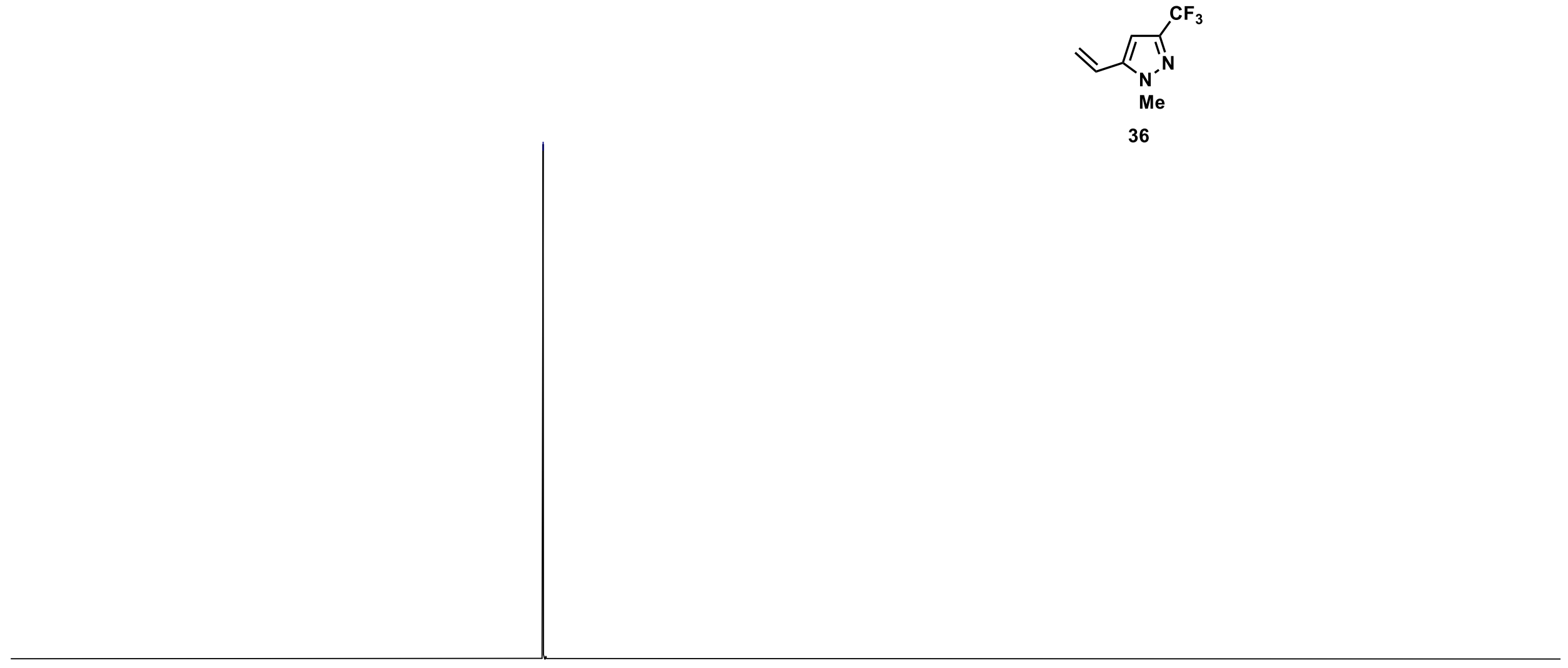


${ }^{1} \mathrm{H}$ NMR of 1-chloro-4-(trifluoromethyl)-2-((2-vinylbenzyl)oxy)benzene (37)

$\mathrm{CDCl}_{3}, 298 \mathrm{~K}$

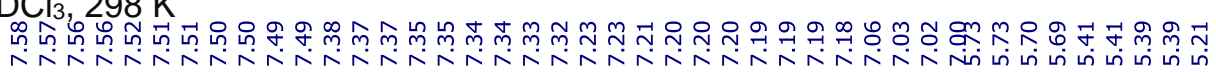

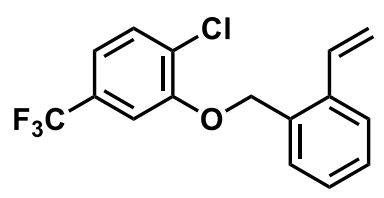

37

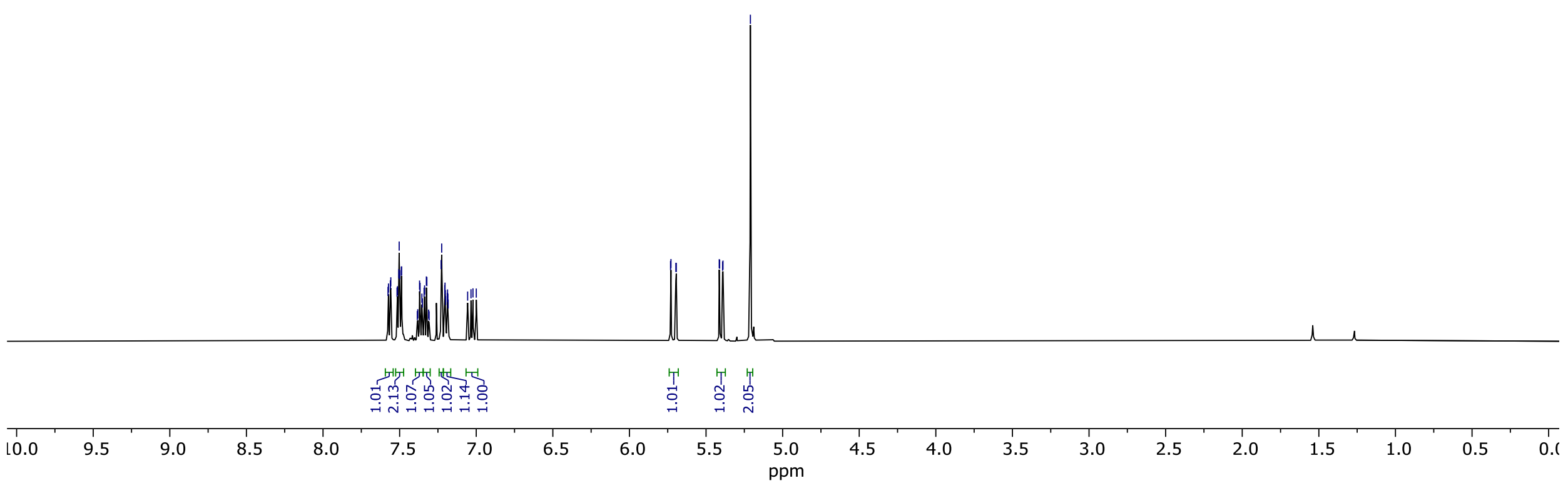


${ }^{13} \mathrm{C}$ NMR of 1-chloro-4-(trifluoromethyl)-2-((2-vinylbenzyl)oxy)benzene (37)

$\mathrm{CDCl}_{3}, 298 \mathrm{~K}$

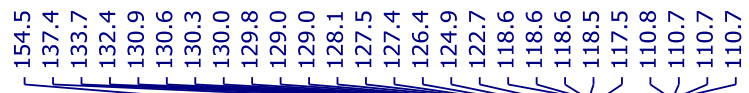

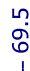

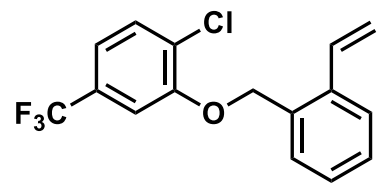

37

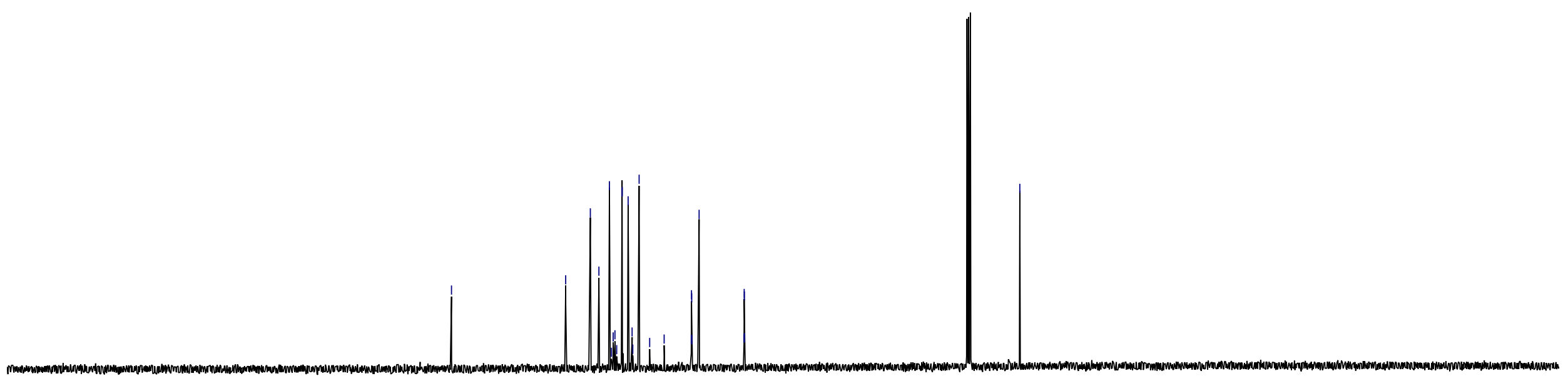


${ }^{19} \mathrm{~F}$ NMR of 1-chloro-4-(trifluoromethyl)-2-((2-vinylbenzyl)oxy)benzene (37)

$\mathrm{CDCl}_{3}, 298 \mathrm{~K}$

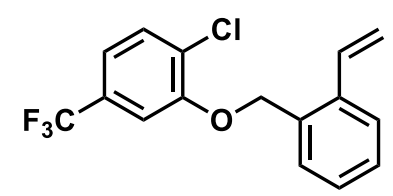

37

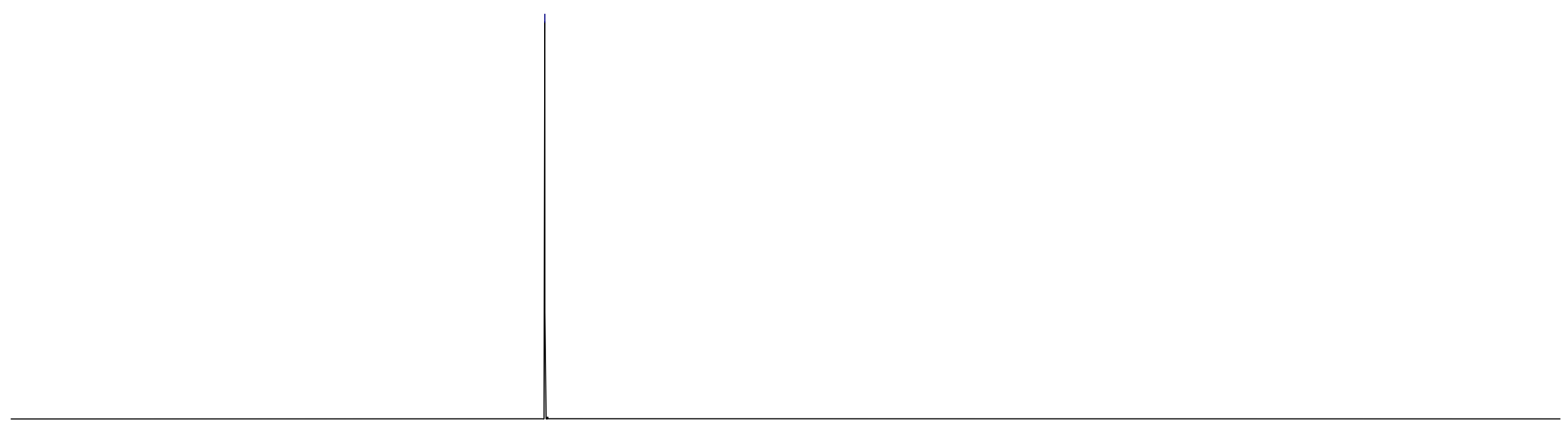

\begin{tabular}{llllllllllllllllllllllllll}
\hline 20 & 10 & 0 & -10 & -20 & -30 & -40 & -50 & -60 & -70 & -80 & -90 & -100 & -110 & -120 & -130 & -140 & -150 & -160 & -170 & -180 & -190 & -200 & -210 & -2 & 1
\end{tabular} 
${ }^{1} \mathrm{H}$ NMR of piperidin-1-yl(4-vinylphenyl)methanone (38)

$\mathrm{CDCl}_{3}, 298 \mathrm{~K}$

我

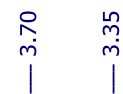

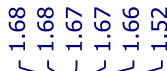

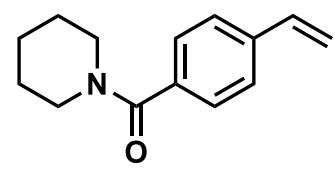

38

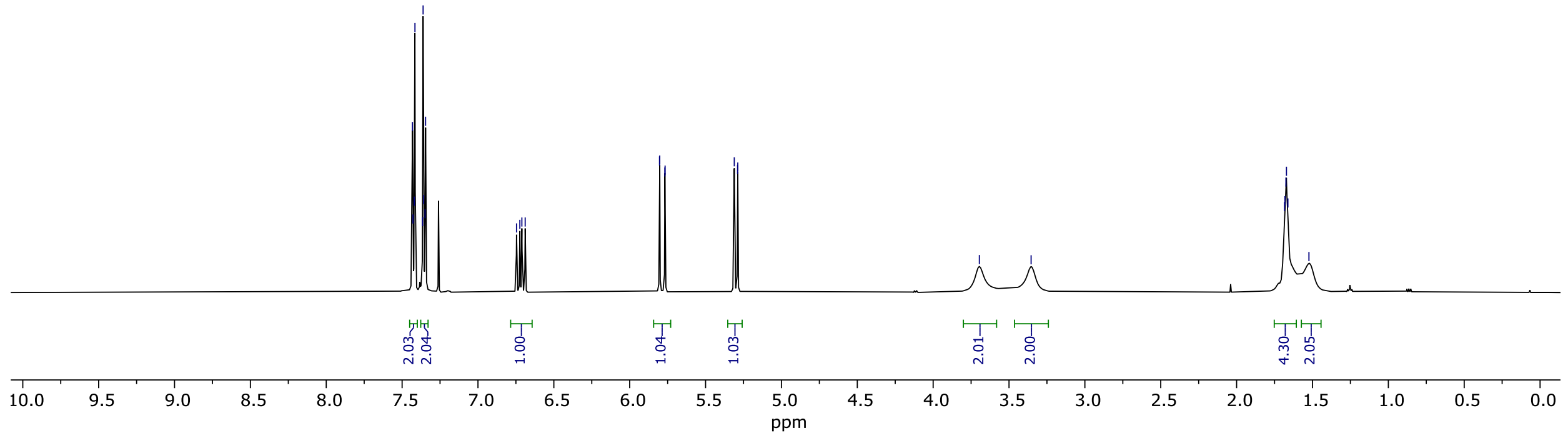


${ }^{13} \mathrm{C}$ NMR of piperidin-1-yl(4-vinylphenyl)methanone (38)

$\mathrm{CD}_{3} \mathrm{CN}, 243 \mathrm{~K}$

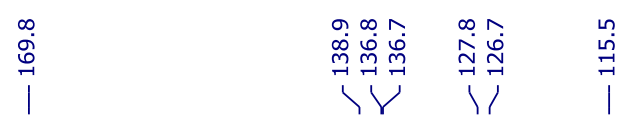

함
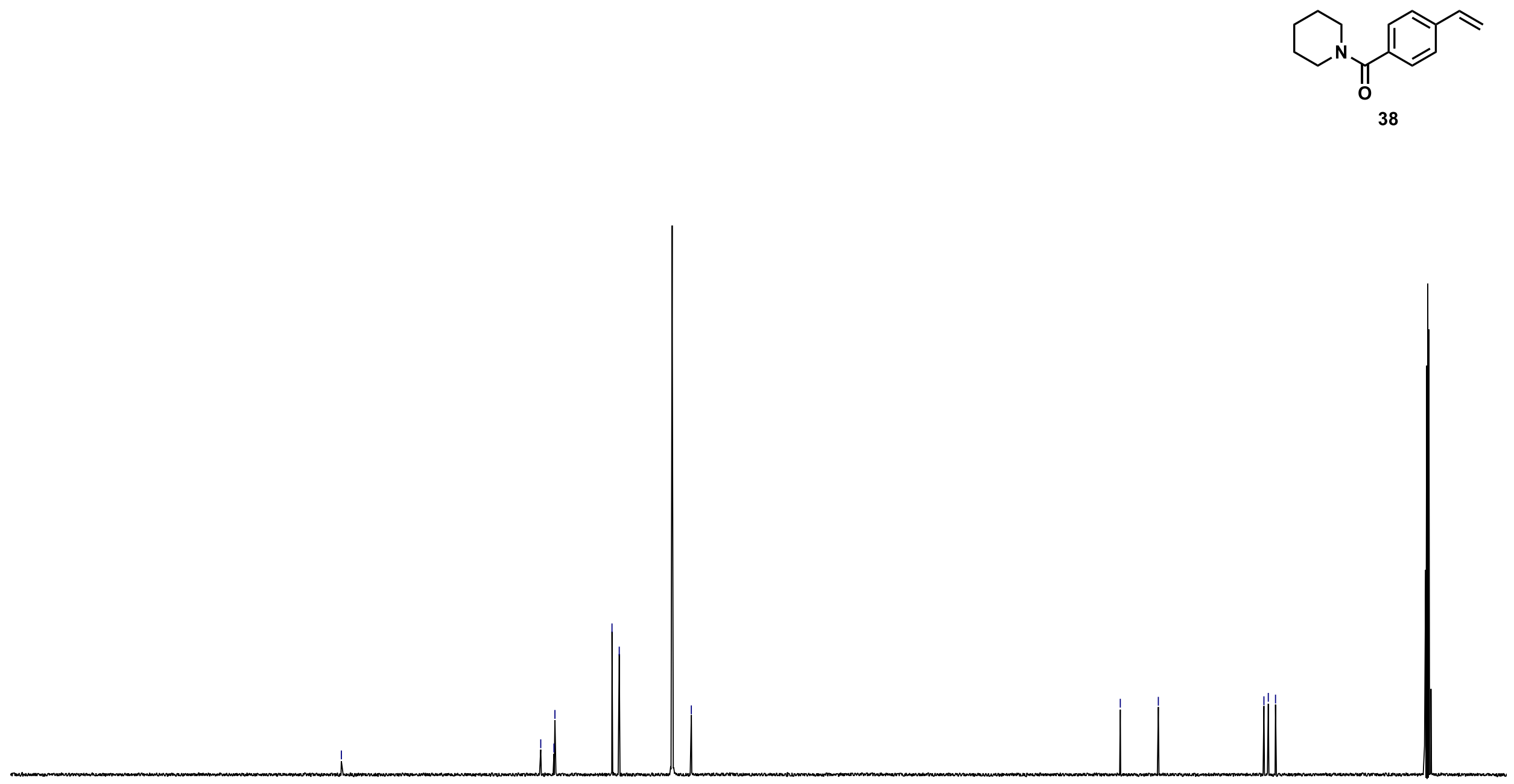

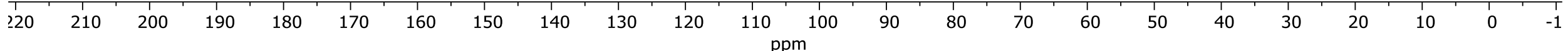


${ }^{1} \mathrm{H}$ NMR of morpholino(3-vinylphenyl)methanone (39)

$\mathrm{CDCl}_{3}, 298 \mathrm{~K}$

每)

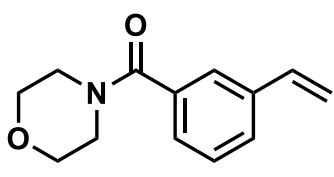

39

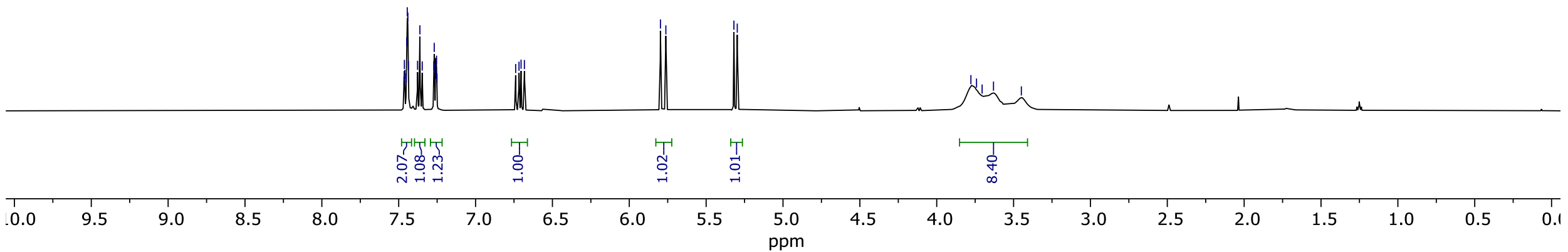


${ }^{13} \mathrm{C}$ NMR of morpholino(3-vinylphenyl)methanone (39)

$\mathrm{CDCl}_{3}, 298 \mathrm{~K}$

焉

$\stackrel{i}{i}$

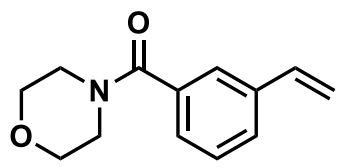

39

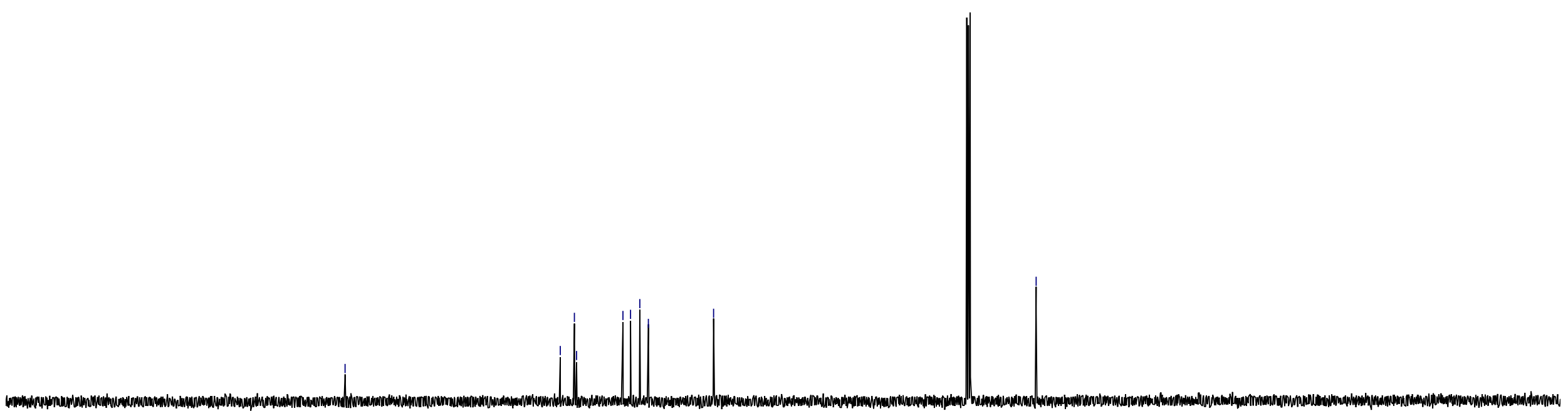


${ }^{1} \mathrm{H}$ NMR of (E)-4-(buta-1,3-dien-1-yl)-1,1'-biphenyl (40)

$\mathrm{CDCl}_{3}, 298 \mathrm{~K}$

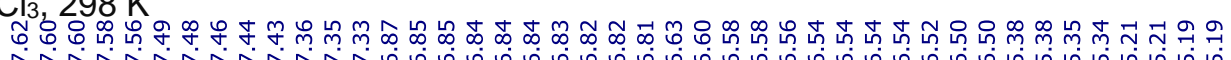
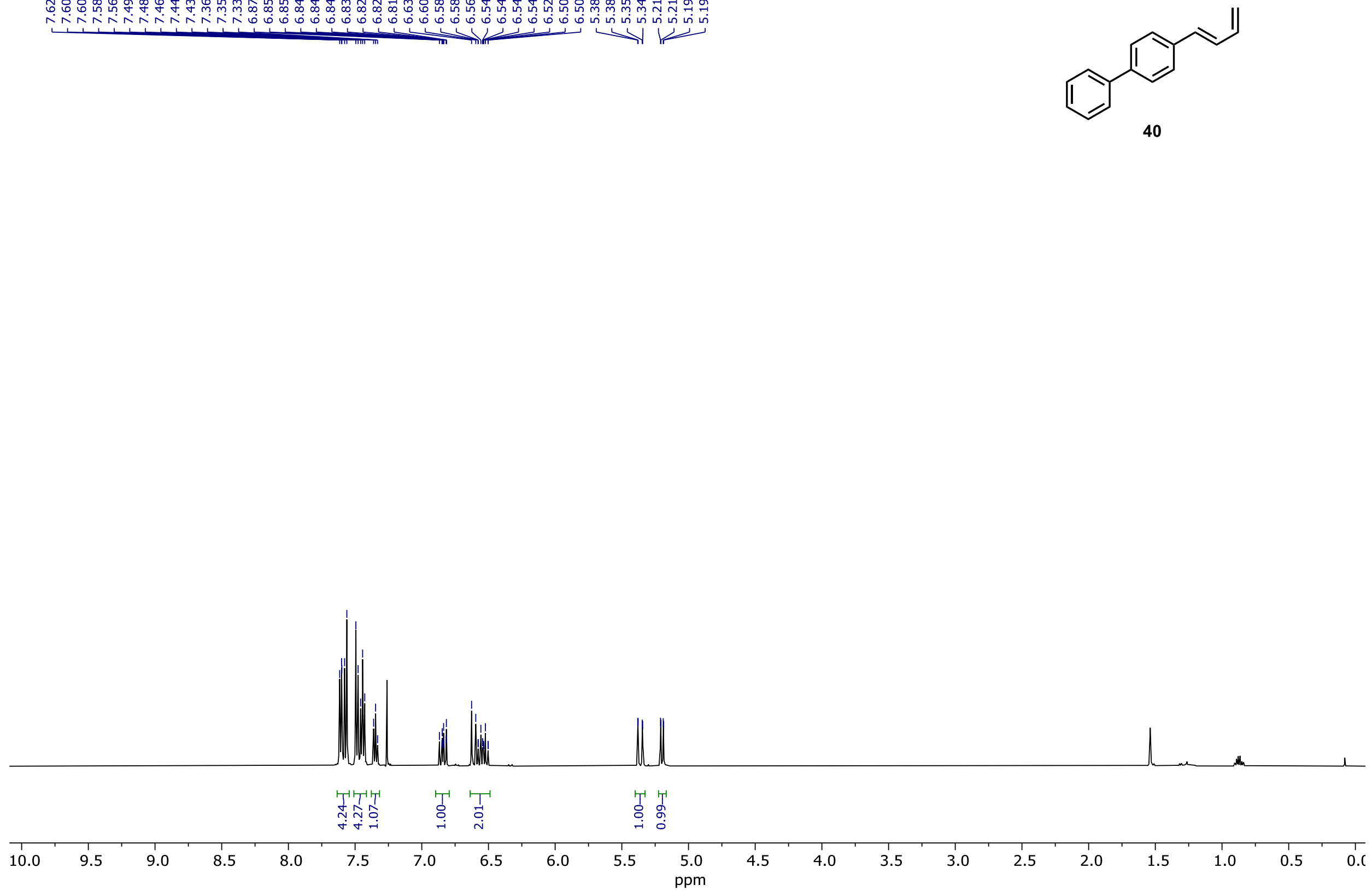
${ }^{13} \mathrm{C}$ NMR of (E)-4-(buta-1,3-dien-1-yl)-1,1'-biphenyl (40)

$\mathrm{CDCl}_{3}, 298 \mathrm{~K}$

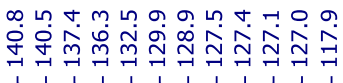

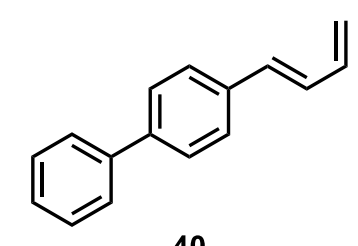

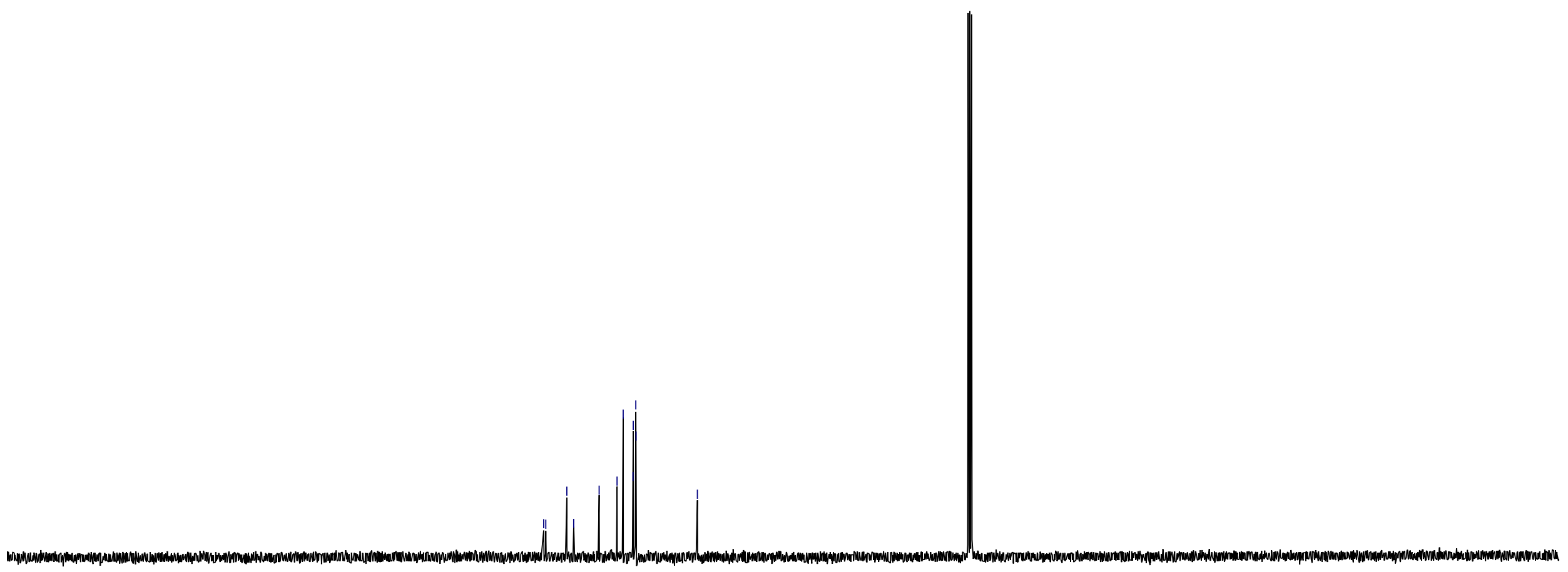


${ }^{1} \mathrm{H}$ NMR of tert-butyl (3-vinylphenyl)carbamate (45)

$\mathrm{CDCl}_{3}, 298 \mathrm{~K}$

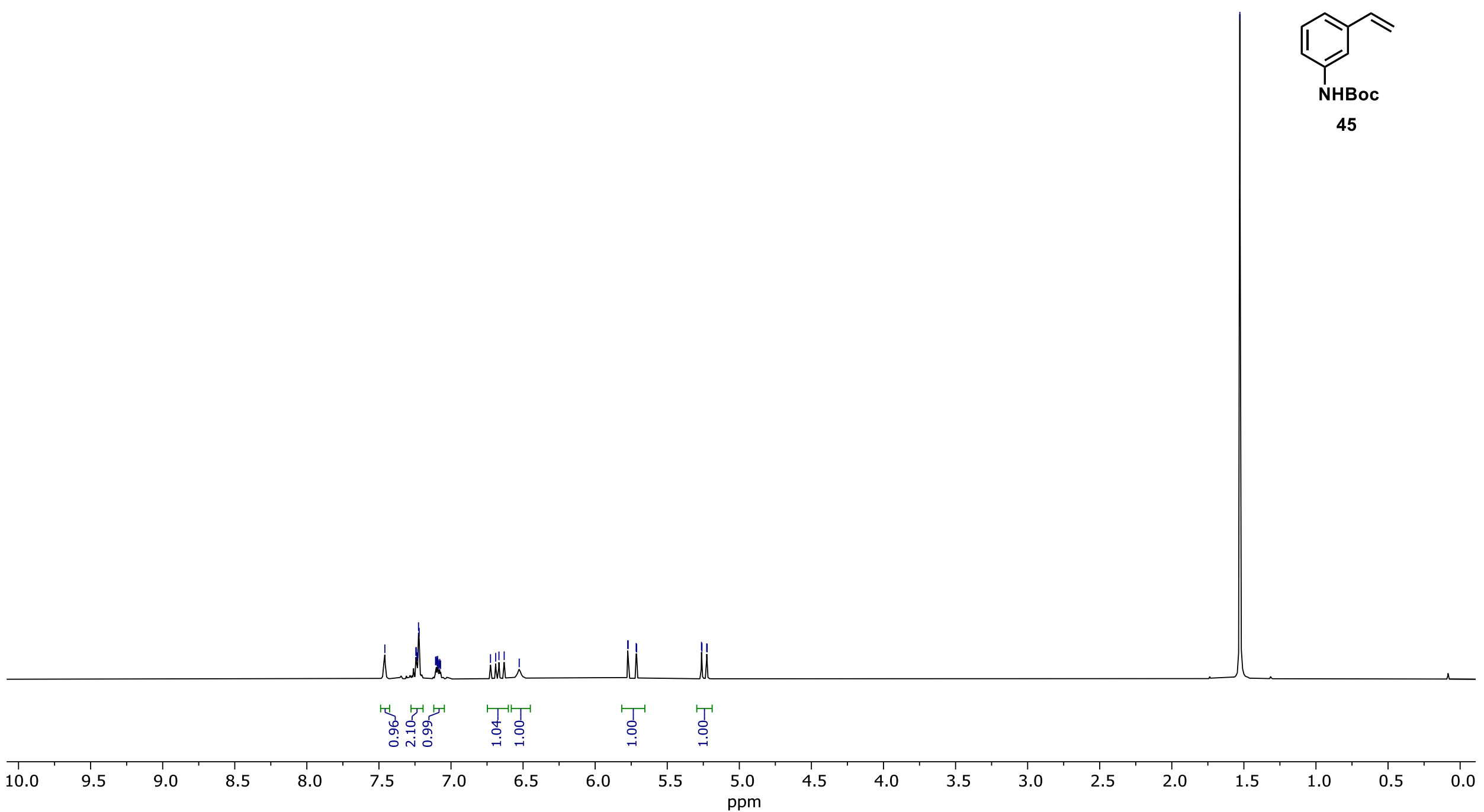


${ }^{13} \mathrm{C}$ NMR of tert-butyl (3-vinylphenyl)carbamate (45)

$\mathrm{CDCl}_{3}, 298 \mathrm{~K}$

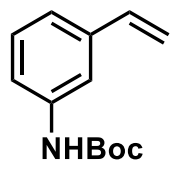

45

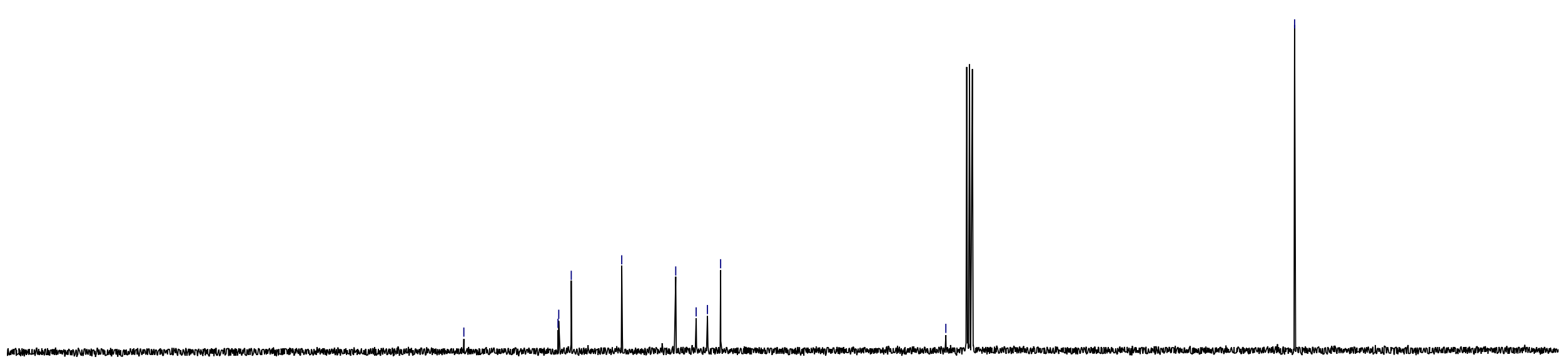


${ }^{1} \mathrm{H}$ NMR of tert-butyl [1,1'-biphenyl]-3-ylcarbamate (46)

$\mathrm{CDCl}_{3}, 298 \mathrm{~K}$

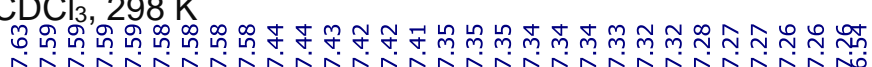

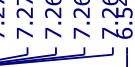

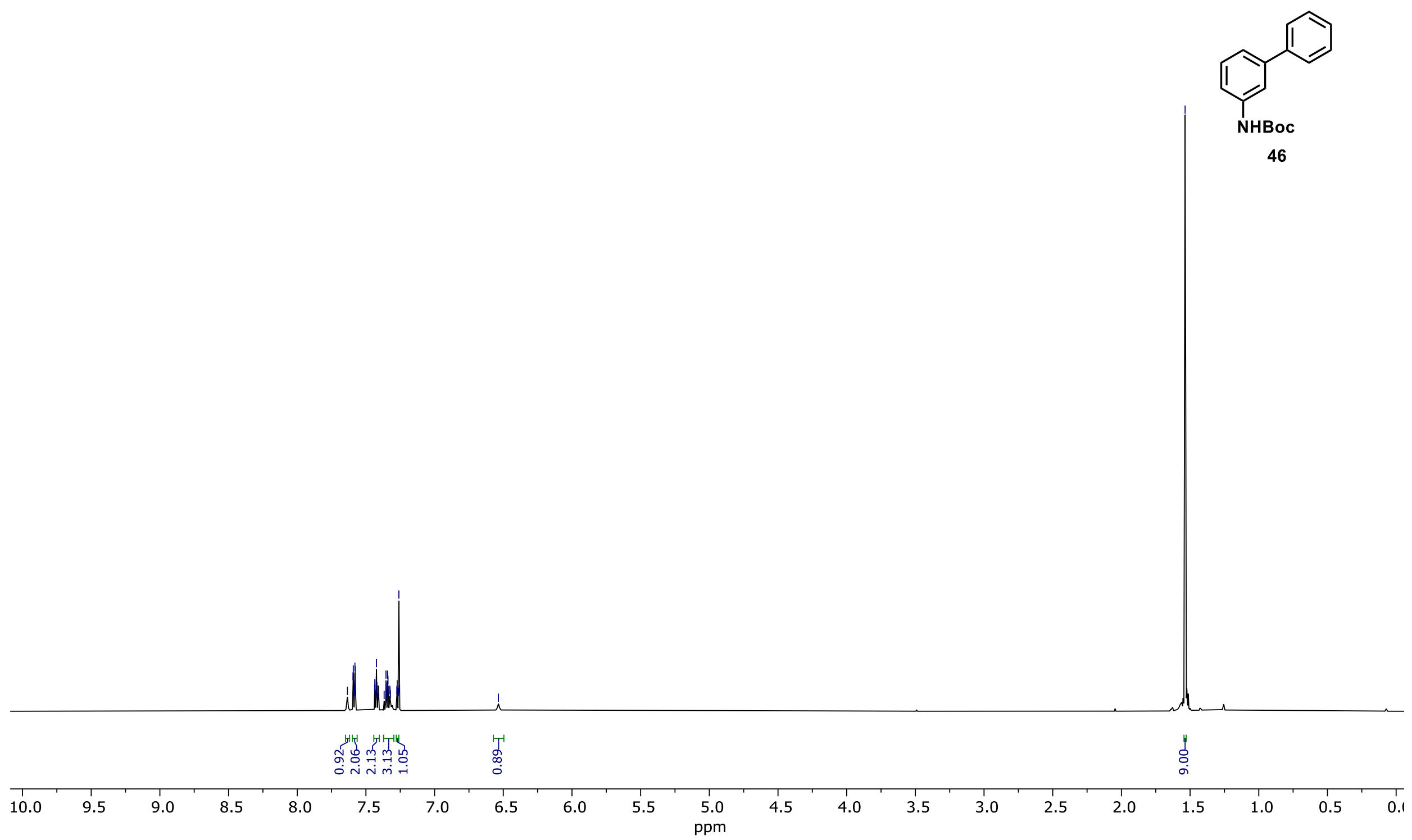


${ }^{13} \mathrm{C}$ NMR of tert-butyl [1,1'-biphenyl]-3-ylcarbamate (46)

$\mathrm{CDCl}_{3}, 298 \mathrm{~K}$

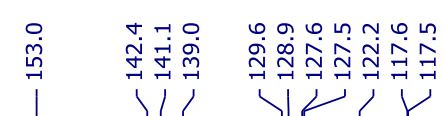

$\stackrel{\infty}{\infty}$

$\stackrel{\substack{\infty \\ \sim}}{\infty}$

$\begin{array}{lll}0 & 11\end{array}$

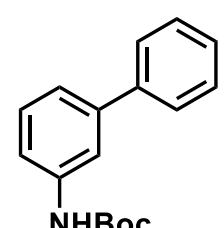

46

$\begin{array}{rrrrrrrrrrrrrrrrrrrrrrrrrr}1 & 210 & 200 & 190 & 180 & 170 & 160 & 150 & 140 & 130 & 120 & 110 & \begin{array}{r}100 \\ \mathrm{ppm}\end{array} & 90 & 80 & 70 & 60 & 50 & 40 & 30 & 20 & 10 & 0 & -10 & \end{array}$




\section{References}

[1] F. Berger, M. B. Plutschack, J. Riegger, W. Yu, S. Speicher, M. Ho, N. Frank, T. Ritter, Nature 2019, 567, 223.

[2] M. Yar, E. M. McGarrigle, V. K. Aggarwal, Angew. Chem. Int. Ed. 2008, 47, 3784.

[3] F. Beltran, I. Fabre, I. Ciofini, L. Miesch, Org. Lett. 2017, 19, 5042.

[4] A. Younai, B.-S. Zeng, H. Y. Meltzer, K. A. Scheidt, Angew. Chem. Int. Ed. 2015, 54, 6900.

[5] H. Dai, M. Yang, X. Lu, Adv. Synth. Catal. 2008, 350, 2

[6] P. Xu, D. Zhao, F. Berger, A. Hamad, J. Rickmeier, R. Petzold, M. Kondratiuk, K. Bohdan, T. Ritter, Angew. Chem. Int. Ed. 2020, 59, 1956.

[7] V. Pfeifer, M. Certiat, D. Bouzouita, A. Palazzolo, S. Garcia-Argote, E. Marcon, D.-A. Buisson, P. Lesot, L. Maron, B. Chaudret, S. Tricard, I. del Rosal, R. Poteau, S. Feuillastre, G. Pieters, Chem. Eur. J. 2020, 26, 4988.

[8] H. G. Roth, N. A. Romero, D. A. Nicewicz, Synlett 2016, 27, 714.

[9] M. Zhou, K. En, Y. Hu, Y. Xu, H. C. Shen, X. Qian, RSC Adv. 2017, 7, 3741.

[10] S. P. Fritz, J. F. Moya, M. G. Unthank, E. M. McGarrigle, V. K. Aggarwal, Synthesis 2012, 44, 1584.

[11] M. G. Unthank, N. Hussain, V. K. Aggarwal, Angew. Chem. Int. Ed. 2006, 45, 7066.

[12] Y. Ye, J. Zhu, Y. Huang, Org. Lett. 2021, 23, 2386.

[13] P. S. Engl, A. P. Häring, F. Berger, G. Berger, A. Pérez-Bitrián, T. Ritter, J. Am. Chem. Soc. 2019, 141, 13346.

[14] E. M. Alvarez, M. B. Plutschack, F. Berger, T. Ritter, Org. Lett. 2020, 22, 4593.

[15] A. Selmani, A. G. Gevondian, F. Schoenebeck, Org. Lett. 2020, 22, 4802.

[16] B. Lansbergen, P. Granatino, T. Ritter, J. Am. Chem. Soc. 2021, 143, 7909.

[17] M. Tobisu, Y. Masuya, K. Babaa, N. Chatani, Chem. Sci. 2016, 7, 2587.

[18] J. Spencer, M. Pfeffer, A. DeCian, J. Fischer, J.Org.Chem. 1995, 60, 1005. 